Luiz Gustavo Dufner de Almeida

\title{
Mutational and functional study of Tuberous Sclerosis Complex 1 and 2 genes (TSC1 and TSC2)
}

Estudo mutacional e funcional dos genes 1 e 2 do Complexo da Esclerose Tuberosa (TSC1 e TSC2)

\section{SÃO PAULO}




\section{Luiz Gustavo Dufner de Almeida}

\section{Mutational and functional study of Tuberous Sclerosis Complex 1 and 2 genes (TSC1 and TSC2)}

Estudo mutacional e funcional dos genes 1 e 2 do Complexo da Esclerose Tuberosa (TSC1 e TSC2)

\section{EXEMPLAR CORRIGIDO - O original encontra-se no Instituto de Biociências da USP}

Tese apresentada ao Instituto de Biociências da Universidade de São Paulo como requisito parcial para obtenção do título de Doutor na área de Biologia/Genética 
Ficha catalográfica elaborada pelo Serviço de Biblioteca do Instituto de Biociências da USP, com os dados fornecidos pelo autor no formulário: http://www.ib.usp.br/biblioteca/ficha-catalografica/ficha.php

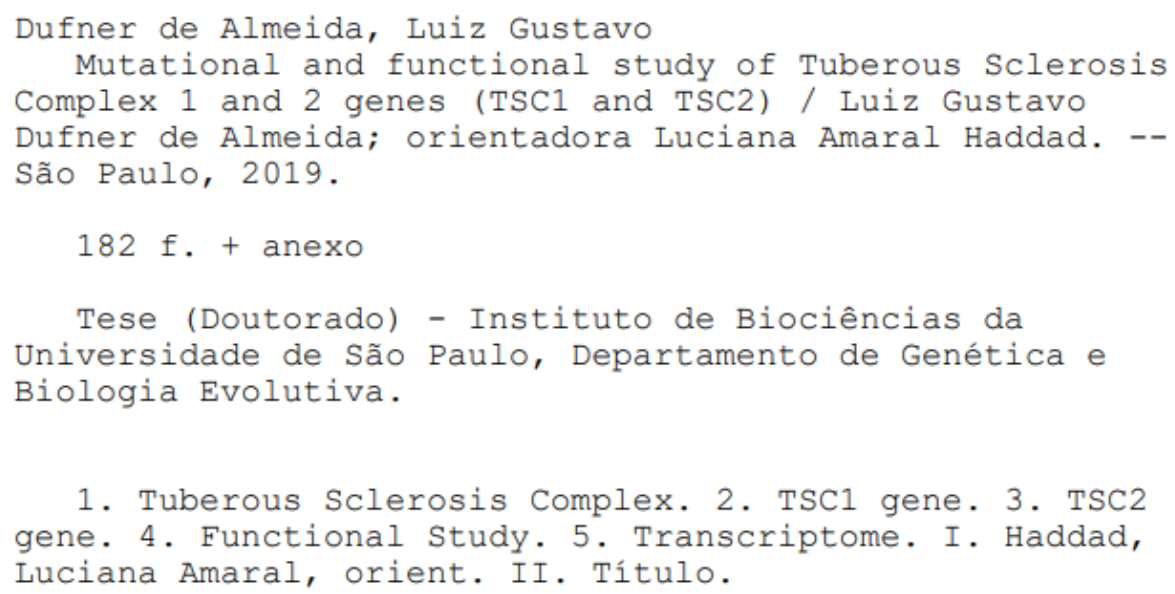

Bibliotecária responsável pela estrutura da catalogação da publicação: Elisabete da Cruz Neves - CRB - 8/6228 
Prof(a) Dr(a) Andrea Laurato Sertie Santos

Prof(a) Dr(a) Maria Isabel A. de S. W. Achatz
Prof(a) Dr(a) Ana Cristina Victorino Krepischi

Prof(a) Dr(a) Paula Fontes Asprino

Prof(a) Dr(a) Luciana Amaral Haddad

Advisor 
Ao meu pai e minha mãe, Luiz Sergio de Almeida e Rosely Christina Dufner de Almeida; meu irmão, Rafael Augusto Dufner de Almeida; minha querida esposa, Karina Campos Tisovec Dufner, e minha filha Sofia Tisovec de Almeida. 


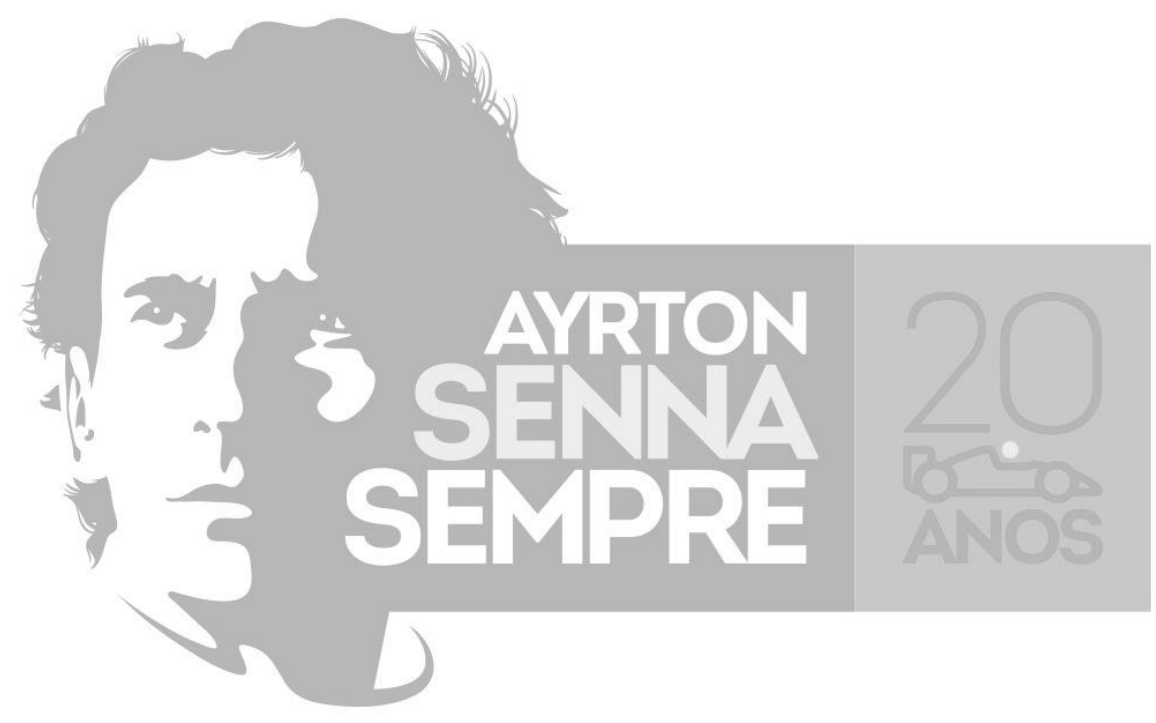

"Eu não kenho idolos. Jenho admiração por trabalho, dedicação e competência"

Qycton Senna da Silva 
This work was financially supported by São Paulo Research Foundation (FAPESP) Research, Innovation and Dissemination Center (RIDC) grant program to Prof. Dr. Mayana Zatz (Human Genome and Stem-Cell Research Center, FAPESP 2013/08028-1). The student was the recipient of the Coordination for the Improvement of Higher Education Personnel studentships CAPES-PROEX/(001) and CAPES-PDSE(88881.132401/2016-01). 


\section{Acknowledgments}

I would like to thank Prof. Dr. Luciana Amaral Haddad, for the encouragement, conviviality and teaching during the eight years in her laboratory, from advisory of my Master's degree to the conclusion of doctorate.

I also would like to thank Dr. Mark Nellist for having me at his laboratory at the Functional Unity on Erasmus Medical Center, Rotterdam, The Netherlands, for enabling the development of the functional assays of the variants found in the TSC patients and the RNAseq analysis of the TSC1 and TSC2 knockout cell lines in his laboratory.

To Profa. Dr. Maria Rita Passos Bueno of the Department of Genetics and Evolutionary Biology, Institute of Biosciences-USP for collaboration with the next generation sequencing of TSC1 and TSC2 genes and funding through FAPESP/CEPID.

I also thank Dr. Cibele Masotti of the Sírio Libanês, Institute of Education and Research for the analysis of RNAseq data through the RPKM data.

Many thanks to Prof. Dr. Sérgio A. Antoniuk, Dra. Ana Paula A. de Pereira, Dra. Laís F. M. Cardozo, Dra. Mariana R. Schwind and Dra. Danielly S. C. Cândido of the Clinical Hospital Complex of the Federal University of Paraná, for attending and in collecting blood samples from patients with TSC. To Prof. Sérgio Rosemberg, Dra. Juliana Paula and co-workers, thank you for receiving patients with TSC, and the collaboration with our study. From GRAACC-SP, thank Dra. Nasjla Saba and her team for the care of patients with TSC and the collection and sending of blood samples for our research.

Finally, thank the Msc. Thiago Alegria from the Department of Genetics and Evolutionary Biology, Institute of Biosciences-USP and Santoescha Nahoe from the Functional Unit of Erasmus Medical Center, Rotterdam, The Netherlands, for helping me in experimental designs and bench work during the final stages of my doctorate. 


\section{Abstract}

Tuberous sclerosis complex (TSC) is an autosomal dominant disorder caused by pathogenic variants in either TSC1 or TSC2 tumor suppressor genes. It affects more often the brain, skin, kidneys, heart, lungs, and retina. The protein products of both genes, TSC1 (hamartin) and TSC2 (tuberin), interact, assembling a complex that inhibits mTORC1. Cells with bi-allelic inactivation of either TSC1 or TSC2 genes present hyperactivation of mTORC1, which phosphorylates downstream targets, up-regulating cell proliferation and growth. Moreover, a functional role as heat-shock protein (HSP) co-chaperone has been assigned to TSC1 protein. The first aim of the thesis was to analyze the nature, distribution and functional effects of TSC1 and TSC2 DNA variants from 100 patients with definite clinical diagnosis of TSC. We analyzed leukocyte DNA of 117 TSC patients from three Brazilian tertiary referral hospitals. Pathogenic DNA variants were detected in 102 (87.2\%) unrelated individuals; 18 (17.6\%) in TSC1 and 84 (82.4\%) in TSC2. Clear loss-of-function variants were detected in 89 patients, of which frameshift (29.4\%) and nonsense (29.4\%) were the most common types. In-frame deletions, missense and putative splicing DNA variants with uncertain clinical significance (VUS) have been functionally assessed. Eleven variants significantly increased phosphorylation of the reporter residue S6K Thr ${ }^{389}$. Forty-two novel pathogenic DNA variants and 19 novel single nucleotide variants have been detected. Among the 15 individuals with no mutation identified, ten rare alterations classified as VUS were detected and were putative missense, splicing, large deletion or in the 3'-UTR. To understand the regulatory relationship of TSC1/2 gene expression, we aimed to evaluate TSC1 and TSC2 mRNA and protein levels in human cell lines with bi-allelic inactivation of each gene. We employed high-throughput transcriptome analysis (RNA-Seq) and Western blotting of HEK293T and other six HEK293T-derived cell lines that had the genomic sequence of the TSC1 and or TSC2 genes edited by the CRISPR (clustered regularly interspaced short palindromic repeats)-Cas9 system. In lack of either TSC1 or TSC2 protein, a significant reduction of the respective mRNA was observed, inferring no positive transcriptional feedback. Serum-deprived cell lines without TSC1 decreased TSC2 mRNA levels. Under these conditions, TSC1 mRNA levels were not negatively affected by the lack of TSC2. In one cell line with loss of TSC1 (1C2) TSC1/2 mRNA and TSC2 protein levels were consistently decreased independently on serum. RNA-Seq gene ontology analyses comparing 1C2 to HEK293T reference cell line disclosed downregulation of genes from translational pathways independently on serum; and up-regulation of genes related to protein folding and stability pathways upon serum withdrawal. Our data are consistent with the role of TSC1 as HSP co-chaperone, and suggest that TSC1 mRNA may be regulated at both transcriptional and decay levels.

This work was conducted with financial support from Conselho de Aperfeiçoamento de Pessoal de Nível Superior - Programa de Excelência Acadêmica (CAPES-Proex, 001). 


\section{Resumo}

O complexo da esclerose tuberosa (TSC) é um distúrbio autossômico dominante causado por variantes patogênicas em um de dois genes supressores de tumor TSC1 ou TSC2. Afeta mais frequentemente o cérebro, a pele, os rins, o coração, os pulmões e a retina. Os produtos proteicos de ambos os genes - TSC1 (hamartina) e TSC2 (tuberina) - interagem e formam um complexo que inibe o mTORC1. Células com inactivação bi-alélica dos genes TSC1 ou TSC2 apresentam hiperactivação de mTORC1, a qual fosforila alvos a jusante e regula positivamente proliferação e crescimento celular. Um papel funcional como co-chaperona da proteína de choque térmico (HSP) foi atribuído à proteína TSC1. O primeiro objetivo desta tese foi analisar a natureza, distribuição e os efeitos funcionais das variantes de DNA de TSC1 e TSC2 de 100 pacientes com diagnóstico clínico definitivo de TSC. Analisamos o DNA de leucócitos de 117 pacientes com TSC acompanhados em três hospitais brasileiros de atenção terciária. Variantes patogênicas de DNA foram detectadas em 102 (87,2\%) indivíduos não relacionados; 18 (17,6\%) em TSC1 e 84 (82,4\%) em TSC2. Variantes patogênicas de perda de função foram detectadas em 89 pacientes, dos quais as variantes frameshift $(29,4 \%)$ e nonsense $(29,4 \%)$ foram os tipos mais comuns. Deleções em fase, variantes missense e variantes de splicing com significância clínica incerta (VUS) foram avaliadas funcionalmente. Onze variantes aumentaram significativamente a fosforilação do resíduo Thr ${ }^{389}$ da proteína repórter S6K. Quarenta e duas novas variantes patogênicas e 19 novas variantes de nucleotídeo único foram detectadas. Identificaram-se entre os $15 \mathrm{NMI}$ indivíduos, dez alterações raras, classificadas como VUS, do tipo grande deleção, missense, splicing ou na região 3'-UTR. Para entender a relação regulatória da expressão dos genes TSC1 e TSC2, o segundo objetivo foi avaliar os níveis de RNAm e proteína de TSC1 e TSC2 em linhagens de células humanas com inativação bi-alélica de cada gene. Empregamos uma análise de transcriptoma de alto rendimento (RNA-Seq) e Western blotting de HEK293T e outras seis linhagens celulares derivadas de HEK293T que possuíam a sequência genômica dos genes TSC1 e/ou TSC2 editados por CRISPR-Cas9. Na falta da proteína TSC1 ou TSC2, foi observada uma redução significativa do respectivo RNAm, inferindo ausência de feedback transcricional positivo. Linhagens celulares $\mathrm{TSC1}^{\text {\%- }}$, privadas de soro diminuíram os níveis de RNAm de TSC2. Sob estas condições, os níveis de RNAm de TSC1 não foram afetados negativamente pela falta de TSC2. Numa linhagem celular com a perda de TSC1 (1C2), RNAm de TSC1/2 e proteína TSC2 foram consistentemente diminuídos independentemente do soro. Análise de ontologia gênica de dados de RNAm comparando a linhagem celular de 1C2 com a referência HEK293T revelou uma regulação negativa de genes de vias de tradução independentemente de soro; e, após a retirada do soro, regulação positiva de genes das vias de dobramento e estabilidade de proteínas. Nossos dados são consistentes com o papel do TSC1 como co-chaperona de HSP, e sugerem que o RNAm de TSC1 pode ser regulado ao nível da transcrição.

O presente trabalho foi realizado com apoio da Coordenação de Aperfeiçoamento de Pessoal de Nível Superior - Brasil (CAPES) - Código de Financiamento 001 
This thesis has three chapters. The first one reviews the genetics and diagnostics aspects of tuberous sclerosis complex (TSC). The second chapter presents the results of DNA variant analyses in TSC1 and TSC2 genes in a cohort of 117 patients with definite clinical diagnosis of TSC. The last chapter is centered in the analysis of TSC1 and TSC2 gene expression in human cell lines lacking either protein. As the two aims of the thesis were non-overlapping, chapters 2 and 3 have each a final section where conclusions are presented. 
Chapter I

\section{Genetics and diagnostics of tuberous sclerosis complex (TSC)}




\section{List of Acronyms}

ADPKD: Autosomal dominant polycystic kidney disease

AML: Angiomyolipomas

elF4E: Eukaryotic translation initiation factor $4 \mathrm{E}$

GAP: GTPase-activating protein

HSP: Heat-shock proteins

LAM: Lymphangioleiomyomatosis

MLPA: Multiplex ligation-dependent probe amplification

MMPH: Multifocal, micronodular, type II pneumocyte hyperplasia

mTORC1: Mechanistic target of rapamycin complex 1

PECs: Perivascular epithelioid cells

PKD1: Polycystic Kidney Disease 1 gene

RHEB: RAS-homology enriched in brain

S6K1: p70 40S ribosomal protein S6 kinase B1

SEGA: Subependymal giant cell astrocytoma

SEN: Subependymal nodule

TSC: Tuberous sclerosis complex

TSC1: Tuberous sclerosis complex 1 gene

TSC2: Tuberous sclerosis complex 2 gene

UTR: Untranslated region 


\section{List of Tables}

Table I-1: First clinical cases reported with signs of TSC in the 1860 s to $1880 \mathrm{~s}$

.18

Table I-2: Revised criteria for the diagnosis of TSC according to the International Conference on Consensus on

Tuberous Sclerosis Complex (modified from Northrup et al. (2013)). .19 


\section{List of Figures}

Figure I-1: Map of the human chromosome 16 p13.3 zoomed into 4,130 bp of TSC2 and PKD1 gene 3'-end overlapping region 


\section{Summary}

I. Chapter I .11

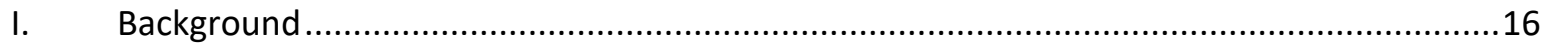

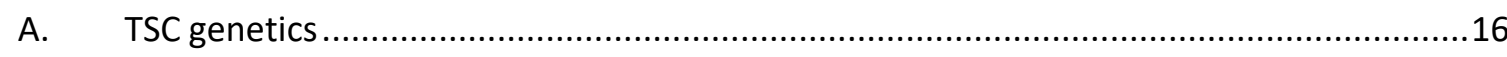

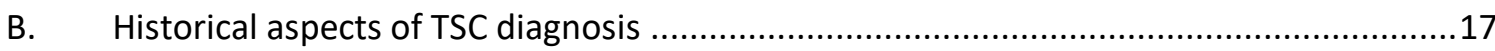

C. TSC lesion classification according to the cell of origin: clinical manifestations and outcomes 19

II. TSC molecular diagnosis and genotype-phenotype correlations ....................................24

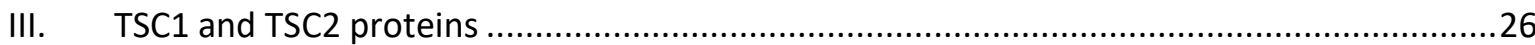

A. TSC2 GAP domain and the mechanistic target of rapamycin (mTOR) pathway ..............26

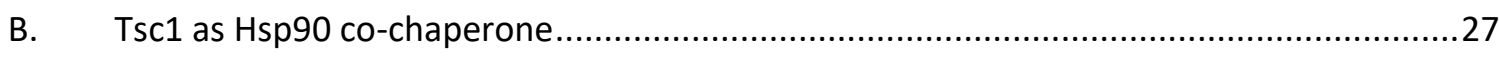

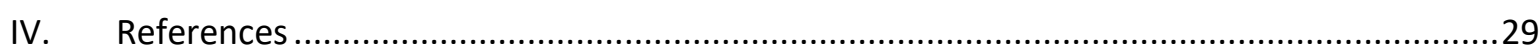




\section{Background}

Tuberous sclerosis complex (TSC) is a clinical disorder characterized as hereditary, multiple system hamartomatosis. TSC hamartomas can affect any organ, although most commonly the brain, heart, kidneys, lungs, retina and skin. It has an estimated incidence between 1:10,000 and 1:6,000 live born infants, and no ethnic bias (Sampson, Scahill et al. 1989, O'Callaghan, Shiell et al. 1998, Au, Williams et al. 2007).

The term hamartoma, suggested by Albrecht in 1904, defines slow-growing benign tumors of cells that normally occur in the tissue of origin, arranged in a disorganized, though circumscribed, non-encapsulated manner (Cooper 1971). TSC specific organ hamartomas classify as infrequent histological types, and in general have onset in a defined developmental period. Clinical manifestations usually occur due to organ dysfunction as a result of tissue architecture rupture or compressive or vascular phenomena (Roach and Sparagana 2004, Franz, Bissler et al. 2010, Grajkowska, Kotulska et al. 2010, Krueger, Northrup et al. 2013, Northrup, Krueger et al. 2013). Although neoplasms are not TSC hallmarks, they may uncommonly occur at earlier age than in the general population, in specific organs, notably the kidneys.

Two characteristics are frequently shared by TSC hamartomas: (i) the increased size of the affected cells, which are commonly described as giant cells; and (ii) the challenge to classify the cell type by immunolabeling with specific antibodies (Huttenlocher and Wollmann 1991). Hence, it has been proposed that TSC hamartomas stem from deficiencies in cell size, proliferation and differentiation control.

\section{A. TSC genetics}

TSC is an autosomal dominant disorder caused by germline pathogenic variants in either the TSC1 or TSC2 tumor suppressor genes (Consortium 1993, van Slegtenhorst, de Hoogt et al. 1997). Molecular studies of TSC hamartomas resected from mouse models of the disease or from patient surgery specimens have mostly agreed with Knudson's second-hit hypothesis (Niida, Stemmer-Rachamimov et al. 2001), which has been established as a genetic model for tumor suppressor genes (Knudson 1971). It currently predicts that a somatic cell harboring a germline mutation will present tumor clonal properties if the wild-type allele is inactivated by a pathogenic DNA variant or epigenetic alteration. The randomness and uncertainty of the second alleleinactivating hit are believed to contribute to decrease the penetrance of the disease. TSC incomplete penetrance has been reported (Connor, Stephenson et al. 1986, Webb and Osborne 1991). However, recent technological developments have estimated the penetrance of this disease to be, though incomplete, significantly higher than previously observed (Hasbani and Crino 2018). As presented below, TSC clinical diagnostics is based on lesion identification and not on symptomatic clinical presentation (Northrup et al., 2013). Therefore, the increase in population coverage of medical imaging has allowed for the definite clinical diagnosis in asymptomatic patients. Moreover, molecular analysis breakthroughs and the availability of 
parental DNA testing have contributed to increase the estimates of the disease penetrance rates (Caylor, Grote et al. 2018). On the other hand, factors that may negatively influence the penetrance of TSC are its clinical heterogeneity, later age at onset for specific organ hamartomas of the adulthood, and TSC1 and TSC2 genotype-phenotype correlations (Northrup, Wheless et al. 1993, Camposano, Greenberg et al. 2009, von Ranke, Faria et al. 2017). Remarkably, nearly $70 \%$ of TSC cases have been reported as de novo, due to a novel mutation that may be in somatic mosaicism. Consequently, inconclusive diagnosis may implicate in difficulty to reconcile clinical data and mutation segregation studies in families. Finally, familial cases tend to have higher penetrance (Kwiatkowski 1994).

\section{B. Historical aspects of TSC diagnosis}

TSC was first described in 1862 by Friedrick D. von Recklinghausen after the autopsy of a newborn patient disclosed cardiac tumors and malformations of the brain surface. A similar case was later reported in 1864 by Rudolph Virchow. However, it was only in 1880 that the French physician, Désiré Magloire Bourneville, coined the name of the disease as tuberous sclerosis of the cerebral convolutions upon description of autopsied cerebro-cortical malformations that presented tuber-like morphology and consistency firmer than the adjacent parenchyma. The patient had epilepsy, as well as facial and renal lesions (Bourneville 1880, Gomez, Sampson et al. 1999, Jansen, van Nieuwenhuizen et al. 2004) (Table I-1).

Taken together, these descriptions and four other clinical cases reported until 1890 (Table I-1) present clinic-pathological aspects of a disorder that appeared (i) to be systemic, affecting the brain, skin, heart and kidneys; (ii) to be presented as a variable combination of signs, not necessarily observed in all patients; and (iii) to have cortical tubers as the commonest lesion. TSC is also classified as a phacomatosis (from the Greek word phakos, birthmark), a clearly congenital condition associated with a pathological birthmark(McCall, Chin et al. 2006), or as neurocutaneuos syndrome, an inherited condition with major involvement of both, brain and skin. 


\begin{tabular}{|c|c|c|c|c|c|c|c|}
\hline 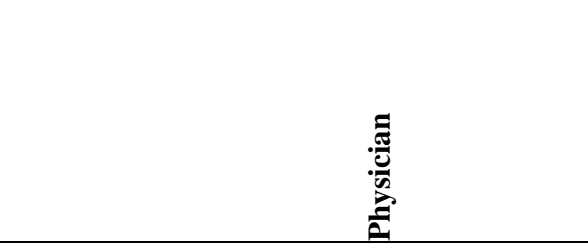 & 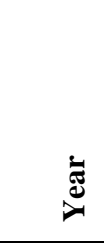 & 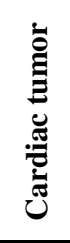 & 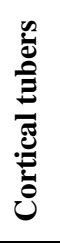 & 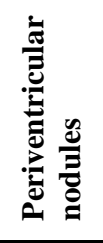 & 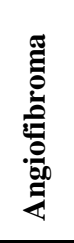 & 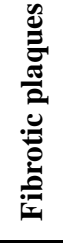 & 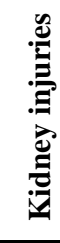 \\
\hline Friedrick D. von Recklinghausen & 1862 & $\sqrt{ }$ & $\sqrt{ }$ & & & & \\
\hline Rudolph Virchow & 1864 & $\sqrt{ }$ & $\sqrt{ }$ & & & & \\
\hline Désiré Magloire Bourneville & 1880 & & $\sqrt{ }$ & & $\sqrt{ }$ & & $\sqrt{ }$ \\
\hline Hartdegen & 1881 & & $\sqrt{ }$ & $\sqrt{ }$ & & & \\
\hline Bourneville and Brissaud & 1881 & $\sqrt{ }$ & $\sqrt{ }$ & $\sqrt{ }$ & & & $\sqrt{ }$ \\
\hline François H. Hallopeau and Émile Leredde & 1885 & & $\sqrt{ }$ & & $\sqrt{ }$ & & \\
\hline John J. Pringle & 1890 & & $\sqrt{ }$ & & $\sqrt{ }$ & $\sqrt{ }$ & \\
\hline
\end{tabular}

$\checkmark$ : Observed phenotype

In 1908, Vogt proposed a clinical triad consisting of sebaceous adenoma (angiofibroma), intellectual disability and epilepsy for the diagnosis of TSC. The development and improvement of medical imaging techniques expanded the knowledge on the disease, leading to the first classification of lesions into major and minor criteria for TSC diagnosis by Gómez in 1979. TSC diagnosis criteria were later reviewed twice by medical experts (Roach, Gomez et al. 1998, Northrup, Krueger et al. 2013). The currently adopted diagnostics criteria are presented in (Northrup, Krueger et al. 2013). The combination of major and minor criteria may yield a definite or possible clinical diagnosis. It is important to notice that each lesion defined as a criterion can occur isolate in a patient, due to two somatic mutation hits. Thus, the adherence to these guidelines is of utmost importance in the clinical setting. Moreover, the latest review board has included the genetic diagnostic criteria, by which the detection of a pathogenic variant in either TSC1 or TSC2 genes is sufficient for the definite diagnosis of TSC. Genetic variants of TSC1 or TSC2 not confirmed as pathogenic are not a criterion for the diagnosis (Table I-2). 
Table I-2: Revised criteria for the diagnosis of TSC according to the International Conference on Consensus on Tuberous Sclerosis Complex (modified from Northrup et al. (2013)).

\section{A. Genetic diagnostic criteria} of $\operatorname{TSC} *$.

The identification of a pathogenic mutation in the $\mathbf{T S C 1}$ or $\boldsymbol{T S C 2}$ gene in normal tissue is enough for the definitive diagnosis

\section{B. Clinical diagnostic criteria}

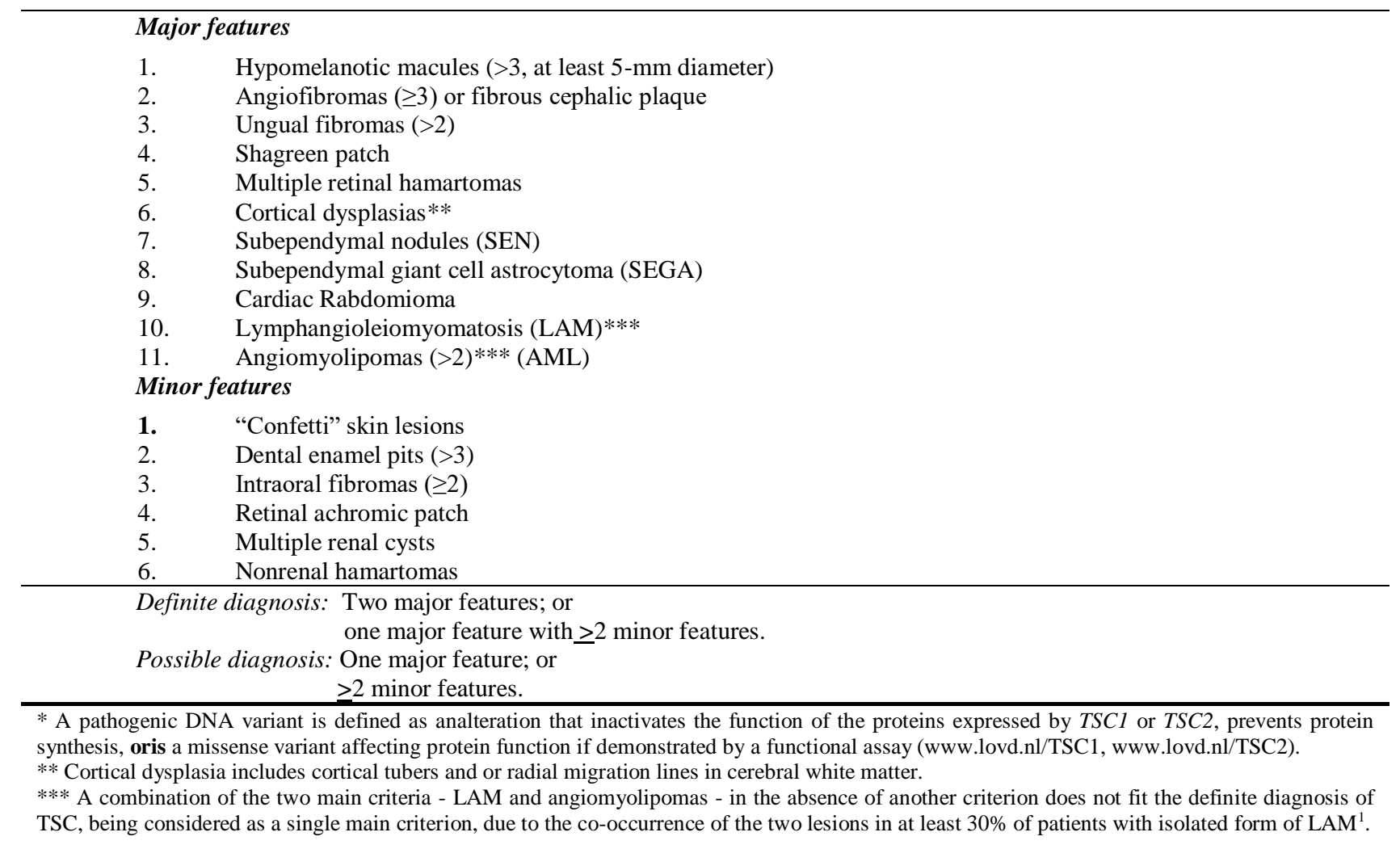

\section{TSC lesion classification according to the cell of origin: clinical manifestations and outcomes}

TSC lesions can be classified in different ways; according to organ or system affected, histological diagnosis subtyping, following clinical manifestations, etc. Here we divide TSC into five large groups according to the pathogenesis and cell of origin.

\section{Central nervous systems (CNS) lesions: TSC as a neurodevelopmental disorder}

Nearly $80 \%$ of patients with TSC will present subependymal nodules (SEN, (Houser and Gomez 1992)), 5 -mm nodules in the subependymal layer of the lateral cerebral ventricles protruding into their cavity, with a smooth, firm and hardened surface, due to frequent calcification. They are lined by a relatively intact ependymal epithelium. Giant cells, with large, convoluted nuclei tend to be observed inside the nodule. They are highly vascular lesions, with vessels prone to hyalinization (Trombley and Mirra 1981, Scheithauer and 
Reagan 1999). Internal hemorrhage and necrosis are rare but may be observed in larger nodules. In $5 \%$ to $15 \%$ of patients with TSC, SENs may progress to subependymal giant cell astrocytoma (SEGA), especially in the first two decades of life, a hypothesis supported by follow-up imaging tests, histological similarities between these hamartomas and their encephalic location (Roach and Sparagana 2004, Grajkowska, Kotulska et al. 2010). The majority of SEGAs are solitary, discrete masses, larger than $5 \mathrm{~mm}$ in diameter and located near the foramen of Monro.

SENs and SEGAs are hamartomas formed by dysplastic astrocytes and neuronal cells (Gomez, Sampson et al. 1999, Jozwiak, Jozwiak et al. 2005). Despite debates on their origins (Davidson, Yoshidome et al. 1991, Johnson, Yoshidome et al. 1991), and although classified as astrocytomas, it is now recommended they should be classified as tumors of mixed origin due to the neuroglial composition of SEGAs (Buccoliero, Franchi et al. 2009). This composition suggests that SEN and SEGA should originate from neuroglial progenitors of the subventricular zone of the brain (Crino 2013). Although SEGA is a tumor classified as grade I astrocytoma (World Health Organization), it may maintain a slow growth rate, at most $2 \mathrm{~mm}$ per year. This growth and its location may contribute to hydrocephalus and acute intracranial hypertension, due to obstructive phenomena. Vascular calcification and hyalinization contribute to the rare occurrence of intramural hemorrhage. Calcification of the stroma is more common in SEN than in SEGA (Huttenlocher and Wollmann 1991, Shepherd, Scheithauer et al. 1991, Stefansson 1991, Scheithauer and Reagan 1999).

Cortical tubers are lesions present at birth in most patients with TSC. However, its observation by magnetic resonance imaging may be impaired in the first two years of life, a period necessary for myelination, which increases the contrast for the identification of these lesions (Houser and Gomez 1992, Shepherd, Houser et al. 1995). Cortical tubers consist of circumscribed enlargement of cerebral gyri in the cerebral parenchyma, with cortical and subcortical location, presenting itself firmer than the adjacent parenchyma.Their slight protrusion in the cerebral parenchyma creates tubercle-like appearance (tuber). Therefore, the term tuberous sclerosis was coined to name the disease.

Neuronal heterotopias result from dissynchrony in neuronal migration along the radial glial fibers (Roach and Sparagana 2004). They present as radial migration lines of the white matter, possibly formed by groups of post-mitotic neuron soma that did not complete the radial migration process (Northrup, Krueger et al. 2013, van Eeghen, Teran et al. 2013). Cortical tubers and neuronal heterotopias are collectively named dysplasias. Since they are composed of non-proliferative cells, they classify as hamartias, and not hamartomas (Huttenlocher and Wollmann 1991, Scheithauer and Reagan 1999).

Cortical tubers and neuronal heterotopias are associated with epilepsy, often refractory to anticonvulsant medication. They may associate with intellectual disability, autism and other types of behavioral disorders. In most patients younger than two years, seizures manifest as infantile spasms, which if not fully 
controlled, may progress to other types of seizures, and associate with autism and intellectual disability (Chiron, Dumas et al. 1997, Hancock and Osborne 1998, Curatolo, Seri et al. 2001, Thiele 2004, Adriaensen, Schaefer-Prokop et al. 2009, Chu-Shore, Major et al. 2010). The lack of therapeutic control of epilepsy in the first five years of life (60-80\% of patients with TSC - (Orlova and Crino 2010)) appears to relate to later severity of intellectual disability (Joinson, O'Callaghan et al. 2003, Humphrey, Williams et al. 2004). Although a correlation between the number and location of cortical tubers and clinical severity has not been convincingly established, patients with temporal distribution of tubers seem to develop more severe epilepsy and behavioral problems (Goodman, Lamm et al. 1997, Hosoyaa, Naitoa et al. 1999, Holmes, Stafstrom et al. 2007). The absence of clinical manifestations due to cortical tubers in the first five years of life is related to a better cognitive and social function in this period and in successive phases of development (Joinson, O'Callaghan et al. 2003, Humphrey, Williams et al. 2004).

The location of each of these four brain lesions (SEN, SEGA, neuronal heterotopias and cortical tubers), commonly observed in the same patient with TSC, suggested they may have a common origin. It has been demonstrated that SEN and SEGA present cell proliferation and growth control deficiency, and potentially originate after the embryonic corticogenesis period from subventricular zone progenitor cells, in which a second, somatic TSC1 or TSC2 mutation is expected. Early in embryonic development (end of the first third of human gestation), post-mitotic neurons generated from neuroglial progenitors start migrating to initiate corticogenesis. Post-mitotic migrating neurons, possibly with cell dysfunction in adhesion and migration processes (Lamb, Roy et al. 2000, Goncharova, Goncharov et al. 2004) may prematurely abrogate cell migration, and accumulate, forming neuronal heterotopias. Neurons that may reach the appropriate cortical layer could form cortical tubers due to synaptogenesis defects (Johnson, Yoshidome et al. 1991, Mizuguchi and Takashima 2001, Nishio, Morioka et al. 2001, Crino 2013).

\section{PEComas}

The World Health Organization histological, including immunohistochemical, definition of perivascular epithelioid cell neoplasms (PECs) is mesenchymal tumors of distinct perivascular epithelioid cells. The family of tumors of the PEComa type is diverse. In patients with TSC, PEComas occur in the form of angiomyolipomas (AML) and pulmonary lymphangioleiomyomatosis (LAM) (Martignoni, Pea et al. 2008, Folpe and Kwiatkowski 2010).

PEComas are characterized by their perivascular localization and are often arranged radially around the vascular lumen. Careful analysis of PEComas reveals blood vessels compressed by the tumor wall. They present eosinophilic, clear to transparent cytoplasm, different from smooth muscle cells, which are intensely eosinophilic. Perivascular epithelioid cells can accumulate large amounts of lipids, mimicking adipocytes or lipoblasts, cells normally found distant from the vascular wall (Folpe and Kwiatkowski 2010). 
AML is the most common PEComa, with a prevalence of $0.13 \%$ in adults, being more frequently diagnosed in women than in men. Renal AMLs are seen in $60 \%$ to $80 \%$ of adult patients with TSC. Displaying a higher frequency in adulthood, AMLs notably have age-dependent frequency (Rakowski, Winterkorn et al. 2006). Despite the high frequency among patients with TSC, about $80 \%$ of patients with AML do not have TSC; hence, isolate AML is more common than associated with TSC (Eble 1998).

These lesions are highly vascularized hamartomas of mixed composition, whose cells have characteristics of adipocytes and smooth muscle, also presenting dysmorphic blood vessels. It is now believed that AMLs are derived from perivascular epithelioid cells (Weeks, Malott et al. 1991, Ashfaq, Weinberg et al. 1993), although the nature of these cells is still a matter of debate, suggestive of pericytes or a cell type originating from the neural crest (Fernandez-Flores 2011).

AMLs are usually expansive, infiltrating masses, which can undergo vascular complications such as microthrombi, embolism and bleeding. These tumors are most commonly seen in the kidneys, and may occur in other location, such as in the liver, adrenal glands, pancreas, and pelvis (Crundwell 2004, Orlova and Crino 2010, Yang, Feng et al. 2012, Northrup, Krueger et al. 2013). Although a large proportion of patients with AML are asymptomatic (Folpe and Kwiatkowski 2010), there is a risk of local painful bleeding due to rupture of tumor aneurysms or compression and/or invasion of the renal parenchyma. The likelihood of progression to chronic renal failure is high if AML is not treated (Northrup, Krueger et al. 2013).

Pulmonary LAM is a progressive and often fatal disease caused by proliferation of perivascular epithelioid cells in both lungs, in muscle fibers, distributed around lymphatic vessels, bronchi, interlobar septa, and pleura (Smolarek, Wessner et al. 1998, Folpe and Kwiatkowski 2010). The (i) diffuse and bilateral distribution of LAM in the lungs, (ii) the co-occurrence of AML and isolate LAM, (iii) the demonstration of somatic mutation in the TSC2 gene in both lesions, AML and LAM, from the same patient, and (iv) the identification of circulating cells with the ame aspect of perivascular epithelioid cells found in these hamartomas are all evidences suggesting that LAM results from AML metastasis to the lung (Carsillo, Astrinidis et al. 2000).

Non-TSC pulmonary LAM (70\%) is more common than LAM in association with TSC (30\%) (Smolarek, Wessner et al. 1998, Carsillo, Astrinidis et al. 2000, Franz, Bissler et al. 2010). The first studies indicated that $1 \%$ to $5 \%$ of patients with TSC had LAM (Castro, Shepherd et al. 1995). After the increased use of computed tomography of the thorax, it was revealed that between $25 \%$ and $40 \%$ of female patients with TSC have LAM (Moss, Avila et al. 2001). More recent studies indicate that the frequency of LAM in patients with TSC increases with age and can reach $80 \%$ of female patients at the end of the fourth decade of life. Patients with AML present progressive dyspnea on exertion and recurrent pneumothorax, typically in the third and fourth decades of life (Northrup, Krueger et al. 2013). 


\section{TSC cutaneous signs}

Hypomelanotic macules are frequent signs in patients with TSC, since they occur in $90 \%$ of the patients, often at birth, and are thus useful for the clinical diagnosis. At least three hypomelanotic macules with $5 \mathrm{~mm}$ or more in diameter consist a major criterion for the clinical diagnosis (Northrup, Krueger et al. 2013).

Facial angiofibromas, characterized as erythematous, painful papules, commonly in the malar processes occur in approximately $75 \%$ of patients with TSC. Usually these lesions develop around 2 to 5 years of age, being very evident in the pubertal phase (Northrup, Krueger et al. 2013). Over time, these lesions become larger, with no significant increase in total number, and may extend to the nasolabial folds (Roach and Sparagana 2004).

Ungual fibroids are nodules in the nail bed, usually painful, that can appear upon minor trauma. They have a frequency of $20 \%$ in young patients, and higher than $80 \%$ in adults with TSC (Roach and Sparagana 2004, Northrup, Krueger et al. 2013).

Fibrotic plaques usually occur on the scalp or face in $25 \%$ of TSC patients, or in the form of Shagreen plaques in $50 \%$ of patients. The latter appear as lumbar plaques with an irregular surface with appearance of leather or orange peel, and its clinical presentation most frequently relate relate to TSC (Northrup, Krueger et al. 2013).

\section{Cardiac Rhabdomyomas}

Two-thirds of the fetuses and newborns with TSC have one or more cardiac rhabdomyomas. Although these tumors usually regress in the postnatal period, there may be a second peak of frequency in adolescence. These hamartomas can cause cardiac arrhythmia often in newborns or do not present any clinical sign. Their prenatal or neonatal identification is an early sign of TSC, very useful for the diagnosis as it is highly suggestive of TSC (Roach and Sparagana 2004, Northrup, Krueger et al. 2013).

\section{Epithelial cells hyperplasia}

Hyperplasia is defined as proliferation of cells at rates higher than the average expected for the tissue. In patients with TSC, they occur most commonly in the lungs and kidneys, although they may affect other organs, such as the liver.

Multifocal, micronodular, type $\|$ pneumocyte hyperplasia (MMPH) is a possible pulmonary manifestation in TSC patients. MMPH is characterized by small nodules ( $1 \mathrm{~mm}$ to $10 \mathrm{~mm}$ ), with clear margins, on the wall of the alveoli in both lungs, at a frequency between $2 \%$ and $3 \%$ among TSC patients. MMPH can 
occur independently of pulmonary LAM (Muir, Leslie et al. 1998, Maruyama, Seyama et al. 2001, Hayashi, Kumasaka et al. 2010).

Although renal epithelial cell hyperplasia as single, unilateral or bilateral cysts may occur in patients with TSC, their isolate presentation is very common in the general population, and increases with aging. On the other hand, $5 \%$ of patients with TSC present a genomic deletion of chromosome 16 involving the $3^{\prime}$ portions of the contiguous genes, TSC2 and PKD1 (Polycystic Kidney Disease 1 gene). In these patients, with the TSC-ADPKD contiguous deletion syndrome, there is coexistence of TSC with ADPKD, a condition characterized by bilateral development of multiple renal cysts (Bastos and Onuchic 2011).

\section{TSC molecular diagnosis and genotype-phenotype correlations}

The TSC1 (NG_012386.1, NM_000368.4) gene maps to 9q34.1, has 23 exons and approximately $55 \mathrm{~kb}$. The coding sequence for the 1,164-amino acid TSC1 protein, also known as hamartin (130 kiloDaltons - KD), lies in exons 3 through 23 (van Slegtenhorst et al., 1997). Exon 23 is the largest (5,407 bp) TSC1 exon, contributing to the long length of the $3^{\prime}$-untranslated region (UTR) of its transcript. TSC1 intron 1 is the longest $(9,447 \mathrm{bp})$ intron, followed by introns $8(8,905 \mathrm{bp})$ and $2(6,080 \mathrm{bp})$. The average exon size is $374 \mathrm{bp}$, while the average intron size is $2030 \mathrm{bp}$.

The TSC2 (NG_005895.1, NM_000548.3) gene maps to 16p13.3, has 42 exons and nearly $46 \mathrm{~kb}$ in length. Its coding sequence resides in exon 2 through exon 42, and is responsible for the synthesis of a 1,807amino acid protein (tuberin or TSC2, 180 kiloDaltons). The largest TSC2 exon is 488-bp long (exon 34), and the largest intron has $4,820 \mathrm{bp}$ (intron 16). The average exon size is $134 \mathrm{bp}$, while the average intron size is $855 \mathrm{bp}$ ((Consortium 1993, Jones, Daniells et al. 1997, van Slegtenhorst, de Hoogt et al. 1997, Dabora, Jozwiak et al. 2001, Sancak, Nellist et al. 2005, Au, Williams et al. 2007)).

Since the cloning of the TSC1 and TSC2 genes ((Consortium 1993, van Slegtenhorst, de Hoogt et al. 1997)), TSC mutation detection has been performed by Sanger sequencing of PCR products from different exons of each gene ((Wilson, Ramesh et al. 1996, Jones, Daniells et al. 1997, Au, Rodriguez et al. 1998, Niida, Lawrence-Smith et al. 1999, van Slegtenhorst, Verhoef et al. 1999, Dabora, Jozwiak et al. 2001)). Techniques such as MLPA (multiplex ligation-dependent probe amplification) and microarray comparative genome hybridization have additionally been used to search for TSC1 and TSC2 genomic deletions ((Rendtorff, Bjerregaard et al. 2005, Kozlowski, Roberts et al. 2007, Oyazato, lijima et al. 2011, van den Ouweland, Elfferich et al. 2011)), which correspond to approximately 7\% of TSC-causing mutations (Kozlowski, Roberts et al. 2007). In the last decade, next-generation sequencing (NGS) of leukocyte DNA has been employed to search for TSC1 and TSC2 mutations. Most commonly, both gene coding sequences have been captured for NGS library constructions of whole exome or customized multigene panels (Qin, Kozlowski et al. 2010, Tyburczy, Wang et al. 2014, Nellist, Brouwer et al. 2015, Tyburczy, Dies et al. 2015, Ismail, Rani et al. 2017). 
Sanger or next-generation DNA sequence efforts generally detect pathogenic DNA variants in $80 \%$ to $95 \%$ TSC patients with definite clinical diagnosis. Nearly $70 \%$ of all pathogenic variants are de novo germline or post-zygotic alterations. These novel mutations occur approximately four times more often in the TSC2 than the TSC1 gene. Among the familial cases, TSC1 and TSC2 pathogenic DNA variants have similar frequencies (Wilson, Ramesh et al. 1996, Au, Rodriguez et al. 1998, Jones, Shyamsundar et al. 1999, Niida, Lawrence-Smith et al. 1999, van Slegtenhorst, Verhoef et al. 1999, Astrinidis, Khare et al. 2000, Dabora, Jozwiak et al. 2001, Rendtorff, Bjerregaard et al. 2005, Sancak, Nellist et al. 2005, Hung, Su et al. 2006, Au, Williams et al. 2007, Kozlowski, Roberts et al. 2007, Sasongko, Wataya-Kaneda et al. 2008).

A genotype-phenotype correlation is observed in patients with a contiguous TSC2 and PKD1 gene deletion (Brook-Carter et al., 1994; Longa et al., 1997). The two genes lie at 16p13.3 in a tail-to-tail fashion, displaying their polyadenylation signals $60 \mathrm{bp}$ apart (Figure I-1). The development of polycystic kidney disease in TSC patients is due to gross deletions involving both genes; thus, the phenotype has been termed TSC2/PKD1 contiguous gene deletion syndrome (Brook-Carter et al., 1994).

A

\begin{tabular}{l|l|l|l|l|l|l|l|l|l|l|l|l|l|l|l|l|l|} 
chr 16 & 16p13.3 & & & & & p12.3 & & p12.1 & $16 \mathrm{p} 11.2$ \\
\cline { 2 - 8 }
\end{tabular}

B

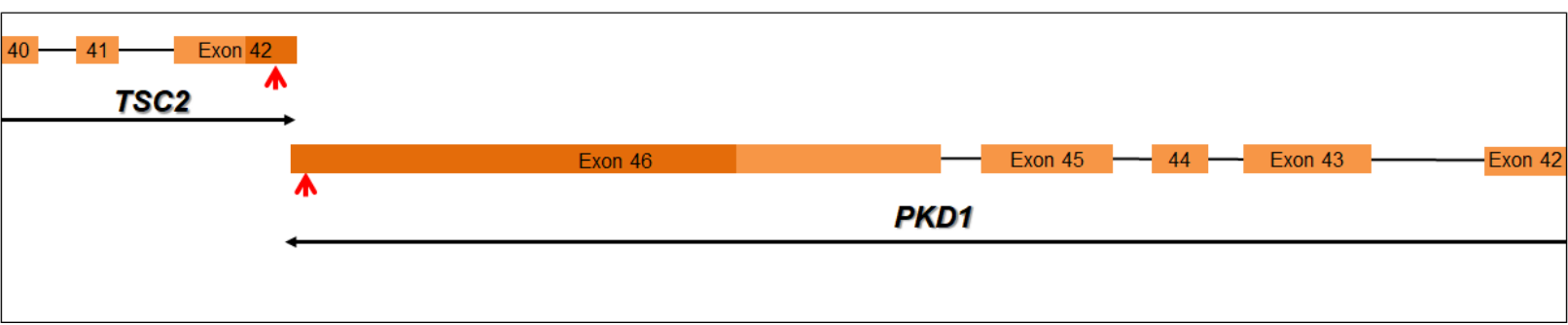

Figure I-1: Map of the human chromosome 16p13.3 zoomed into 4,130 bp of TSC2 and PKD1 gene 3'-end overlapping region. (A) Human chromosome 16. The red bar indicates the location of both genes TSC2 and PKD1 on p13.3 band. (B) Zoom into 4,130 bp relative to the red bar from (A). The direction of TSC2 and PKD1 genes are indicated by thin opposite arrows, as their coding sequences are in different strands of the chromosome 16 DNA. Rectangles represent exons, and lines, introns. Exon numbers from each gene 3 '-end are indicated. Dark orange are untranslated region, and light orange rectangles correspond to the coding sequence. Polyadenylation signals (red thick, short arrows) are $60 \mathrm{bp}$ apart. The drawing of the figure was fully based on and in scale to data retrieved from the genome browser at the University of California in Santa Cruz (Santa Cruz, CA, USA; http://genome.ucsc.edu), according to the GRCh38/hg38 human genome version. Accessed in April, 2019.

Although TSC patients without PKD1 gene alterations may develop renal cysts, the histopathological features appear different in the contiguous gene deletion syndrome (Consugar, Wong et al. 2008). Information on kidney imaging data is helpful for genetic service decision on the choice of DNA test, as MLPA analysis has currently a relatively lower cost, and should be sufficient to detect the genetic cause of the contiguous gene deletion syndrome (Consugar, Wong et al. 2008).

TSC cases classified as mild phenotypes tend to be diagnosed at older age, have more frequently normal cognition, less epilepsy episodes, SEN, SEGA, and sclerotic bone lesions than patients with TSC2 pathogenic variants. Additionally, these individuals tend to show more frequent bilateral and larger renal 
angiomyolipomas than patients with TSC1 mutations. TSC mild phenotypes are more frequently associated with patients with no mutation identified (NMI) or with familial cases (Camposano, Greenberg et al. 2009, Boronat, Barber et al. 2017, Peron, Au et al. 2018).

\section{TSC1 and TSC2 proteins}

TSC1 (NP_000359.1) protein product of TSC1 is a 130-kDa (1164 amino acid) hydrophilic protein with a predicted coiled-coil region of 266 amino acids (residue 730 - 996) close to the C-terminus and an N-terminal domain required for the interaction with TSC2. TSC1 has the ability to form homodimers, demonstrated by the yeast two-hybrid system. TSC1 homodimers are prone to self-aggregation in detergent resistant complexes in the absence of the TSC2 gene product. In the absence of TSC2 overexpression, TSC1 overexpression produces insoluble TSC1, presenting a dotted cytoplasmic distribution at immunofluorescence analysis. If both genes are co-overexpressed, TSC1 becomes soluble and has a more homogeneous cytoplasmic distribution (Nellist, van Slegtenhorst et al. 1999).

TSC2 (NP_000539.2) is a 200-kDa (1,807 amino acid) protein with a short 183-residue region close to the C-terminus that is homologous to the GTPase-activating protein (GAP) for the small GTPase RAP1. This Cterminus domain is encoded by exons 34 to 38 (Maheshwar, Cheadle et al. 1997) and has been shown to act as a GAP for the GTPase RAS Homolog Enriched in Brain (RHEB), catalyzing the RHEB-dependent hydrolysis of GTP to GDP. The N-terminal domain of TSC2 is required for the interaction with TSC1, enabling the two proteins to form a stable complex (Plank, Yeung et al. 1998, van Slegtenhorst, Nellist et al. 1998).

Initially, TSC2 was thought to be localized to the Golgi apparatus, while TSC1 was detected in a punctate pattern in the cytoplasm of cultured cells and localized to a membrane fraction (Plank, Yeung et al. 1998). However, because TSC1 and TSC2 associate physically in vivo, it seemed likely that TSC1 and TSC2 function together in the same complex rather than in separate pathways (van Slegtenhorst, Nellist et al. 1998). It had been suggested that TSC2 could act as TSC1 chaperone, preventing its self-aggregation (Nellist, van Slegtenhorst et al. 1999) maintaining the TSC1-TSC2 complex in a soluble form. The quaternary structure of the TSC complex is not yet clear, but gel filtration and co-immunoprecipitation experiments suggest that the complex contains multiple subunits of both TSC1 and TSC2 (Hoogeveen-Westerveld, van Unen et al. 2012).

\section{A. TSC2 GAP domain and the mechanistic target of rapamycin (mTOR) pathway}

TSC1 and TSC2 form a tumor suppressor complex, the TSC complex, which acts as mediator of intracellular and extracellular signals to inhibit the mechanistic target of rapamycin (mTOR, NP_004949.1) complex 1 (mTORC1) kinase (Garami, Zwartkruis et al. 2003, Tee, Manning et al. 2003). TSC2 acts as GAP 
toward RHEB (Garami, Zwartkruis et al. 2003, Inoki, Li et al. 2003). Inactivation of the TSC complex results in increased levels of RHEB-GTP, that is required for mTORC1 to phosphorylate downstream targets, including p70 40S ribosomal protein S6 kinase 1 (S6K1, NP_003152.1) and eukaryotic translation initiation factor 4E (elF4E) binding protein 1 (4E-BP1, NP_004086.1). Indeed, co-expression of TSC1 and TSC2 represses S6K1 phosphorylation at $\mathrm{Thr}^{389}$, therefore repressing its activity. mTORC1-dependent phosphorylation of 4E-BP1 causes dissociation of $4 \mathrm{E}-\mathrm{BP} 1$ from eIF4E, enabling the configuration of the $48 \mathrm{~S}$ pre-initiation translational complex. Mutants of TSC2 are defective in repressing phosphorylation of 4E-BP1 (Tee, Fingar et al. 2002, Tee, Manning et al. 2003). Taken together, the results implicate the TSC complex in the mTORC1-mediated effects on $\mathrm{S6}$, S6K1 and 4E-BP1, leading in the absence of the active TSC complex to cell over-growth and proliferation, frequent aspects of cell dysfunction in TSC patients. Understanding TSC as an mTOR dysfunction has allowed for clinical trials of rapamycin or analogues (rapalogs) to treat SEGA, AML and LAM. Rapamycin or rapalogs have been clinically approved to control tumor size for these indications in Brazil.

The stability of TSC1-TSC2 complex is central in the control of mTOR signaling. Understanding the regulatory complex of the TSC1 and TSC2 genes is important and may contribute to the selection of vectors for gene therapies in specific TSC lesions (Prabhakar, Zhang et al. 2015). One interesting open question is if the expression regulation of one TSC gene impacts the other TSC gene.

\section{B. Tsc1 as Hsp90 co-chaperone}

Misfolded proteins may aggregate due to the exposure of hydrophobic amino acid residues to the cellular environment. Nearly $30 \%$ of newly synthesized proteins are targeted for degradation due to improper folding (Schubert, Anton et al. 2000). Most proteins require assistance to achieve their correct tertiary structure. Among the systems that have evolved to regulate protein turnover, the most well-known are chaperones. To achieve the correct tertiary and quaternary structure, protein folding factors (chaperones) are required for many proteins (Carlisle, Prill et al. 2017).

When improper protein folding occurs, the heat shock response is activated, and the synthesis of a class of inducible chaperones known as heat-shock proteins (HSP) is increased (Calderwood and Murshid 2017). The molecular chaperone heat-shock protein HSP 90-alpha (Hsp90, NP_001017963.2) is an essential component of the cellular homeostatic machinery in eukaryotes. Purified Hsp90 binds to denatured proteins and displays ATP-dependent anti-aggregate properties (Panaretou, Prodromou et al. 1998). Most Hsp90 clients are medically relevant, including the nuclear receptors for steroid hormones and several protooncogenic kinases and non-kinases.

The ATP-binding pocket in the Hsp90 central domain (residue $272-617$ ) is essential for chaperone activity and to bind and hydrolyze ATP, changing the N-terminal open state to a closed conformation 
(Prodromou and Pearl 2003). The Aha1 co-chaperone aids in high-energy conformational modulations necessary for Hsp90 ATPase competence (Panaretou, Siligardi et al. 2002, Lotz, Lin et al. 2003).

Hsp90 may interact with TSC1 even in the absence of TSC2. The association between TSC2 and TSC1 decreases under thermal shock, which does not affect the association of TSC1-HSP90 (Inoue, Uyama et al. 2010).

Tsc1 has a higher binding affinity for Hsp90 than Aha1. The C-terminal domain of Tsc1 (residue 998 1,164) forms a homodimer that inhibits ATPase activity and stabilizes the closed conformation of the Hsp90 central domain (residues 284- 620 and 728-732) (Brown, Foreman et al. 2015, Woodford, Dunn et al. 2016, Woodford, Sager et al. 2017). Conversely, phosphorylation of Aha1 $\mathrm{Y}^{223}$ increases its affinity for Hsp90 and displaces Tsc1. Because Aha1 and Tsc1 compete for the same domain, Tsc1 may prevent the conformational changes in the catalytic loop of Hsp90 that reduces the release of R400 from its retracted, inactivating conformation, consequently inhibiting Hsp90 ATPase activity (Woodford, Sager et al. 2017). Thus the Hsp90 system switches between an ATP-bound closed conformational state mediated by Tsc1, with low affinity for kinase and non-kinase clients, and an ADP-bound conformational state with high affinity for substrate (Bukau and Horwich 1998, Woodford, Sager et al. 2017).

TSC1 p.L117P naturally occurring human variant located within the N-terminal domain of TSC1 was shown to be pathogenic (Nellist, van den Heuvel et al. 2009). The L117P substitution destabilizes Tsc1 and therefore prevents Tsc2 binding to Hsp90, leading to ubiquitination and degradation by the proteasome (Woodford, Sager et al. 2017), and resulting in increased mTORC1 activity. 


\section{IV.References}

Adriaensen, M. E., C. M. Schaefer-Prokop, T. Stijnen, D. A. Duyndam, B. A. Zonnenberg and M. Prokop (2009). "Prevalence of subependymal giant cell tumors in patients with tuberous sclerosis and a review of the literature." Eur J Neurol 16(6): 691-696.

Ashfaq, R., A. G. Weinberg and J. Albores-Saavedra (1993). "Renal angiomyolipomas and HMB-45 reactivity." Cancer 71(10): 3091-3097.

Astrinidis, A., L. Khare, T. Carsillo, T. Smolarek, K. S. Au, H. Northrup and E. P. Henske (2000). "Mutational analysis of the tuberous sclerosis gene TSC2 in patients with pulmonary lymphangioleiomyomatosis." J Med Genet 37(1): 55-57.

Au, K. S., J. A. Rodriguez, J. L. Finch, K. A. Volcik, E. S. Roach, M. R. Delgado, E. Rodriguez, Jr. and H. Northrup (1998). "Germ-line mutational analysis of the TSC2 gene in 90 tuberous-sclerosis patients." Am J Hum Genet 62(2): 286-294.

Au, K. S., A. T. Williams, E. S. Roach, L. Batchelor, S. P. Sparagana, M. R. Delgado, J. W. Wheless, J. E. Baumgartner, B. B. Roa, C. M. Wilson, T. K. Smith-Knuppel, M. Y. Cheung, V. H. Whittemore, T. M. King and H. Northrup (2007). "Genotype/phenotype correlation in 325 individuals referred for a diagnosis of tuberous sclerosis complex in the United States." Genet Med 9(2): 88-100.

Bastos, A. P. and L. F. Onuchic (2011). "Molecular and cellular pathogenesis of autosomal dominant polycystic kidney disease." Braz J Med Biol Res 44(7): 606-617.

Bourneville, D. M. (1880). "Sclérose tubéreuse des circonvolutions cérébrales." Arch Neurol 1: 11.

Brown, M. A., K. Foreman, J. Harriss, C. Das, L. Zhu, M. Edwards, S. Shaaban and H. Tucker (2015). "C-terminal domain of SMYD3 serves as a unique HSP90-regulated motif in oncogenesis." Oncotarget 6(6): 4005-4019.

Buccoliero, A. M., A. Franchi, F. Castiglione, C. F. Gheri, F. Mussa, F. Giordano, L. Genitori and G. L. Taddei (2009). "Subependymal giant cell astrocytoma (SEGA): Is it an astrocytoma? Morphological, immunohistochemical and ultrastructural study." Neuropathology 29(1): 25-30.

Bukau, B. and A. L. Horwich (1998). "The Hsp70 and Hsp60 chaperone machines." Cell 92(3): 351-366.

Calderwood, S. K. and A. Murshid (2017). "Molecular Chaperone Accumulation in Cancer and Decrease in Alzheimer's Disease: The Potential Roles of HSF1." Front Neurosci 11: 192.

Camposano, S. E., E. Greenberg, D. J. Kwiatkowski and E. A. Thiele (2009). "Distinct clinical characteristics of tuberous sclerosis complex patients with no mutation identified." Ann Hum Genet 73(2): 141-146.

Carlisle, C., K. Prill and D. Pilgrim (2017). "Chaperones and the Proteasome System: Regulating the Construction and Demolition of Striated Muscle." Int J Mol Sci 19(1).

Carsillo, T., A. Astrinidis and E. P. Henske (2000). "Mutations in the tuberous sclerosis complex gene TSC2 are a cause of sporadic pulmonary lymphangioleiomyomatosis." Proc Natl Acad Sci U S A 97(11): 6085-6090.

Castro, M., C. W. Shepherd, M. R. Gomez, J. T. Lie and J. H. Ryu (1995). "Pulmonary tuberous sclerosis." Chest 107(1): $189-195$.

Caylor, R. C., L. Grote, I. Thiffault, E. G. Farrow, L. Willig, S. Soden, S. M. Amudhavalli, A. J. Nopper, K. A. Horii, E. Fleming, J. Jenkins, H. Welsh, M. Ilyas, K. Engleman, A. Abdelmoity and C. J. Saunders (2018). "Incidental diagnosis of tuberous sclerosis complex by exome sequencing in three families with subclinical findings." Neurogenetics 19(3): 205-213.

Chiron, C., C. Dumas, I. Jambaqué, J. Mumford and O. Dulac (1997). "Randomized trial comparing vigabatrin and hydrocortisone in infantile spasms due to tuberous sclerosis." Epilepsy Research 26(2): 389-395.

Chu-Shore, C. J., P. Major, S. Camposano, D. Muzykewicz and E. A. Thiele (2010). "The natural history of epilepsy in tuberous sclerosis complex." Epilepsia 51(7): 1236-1241.

Connor, J. M., J. B. Stephenson and M. D. Hadley (1986). "Non-penetrance in tuberous sclerosis." Lancet 2(8518): 1275.

Consortium, T. E. C. T. S. (1993). "Identification and characterization of the tuberous sclerosis gene on chromosome 16." Cell 75(7): $1305-1315$.

Cooper, J. R. (1971). "Brain tumors in hereditary multiple system hamartomatosis (tuberous sclerosis)." J Neurosurg 34(2 Pt 1): 194-202. 
Crino, P. B. (2013). "Evolving neurobiology of tuberous sclerosis complex." Acta Neuropathol 125(3): 317-332.

Crundwell, M. (2004). "Pathology and genetics of tumours of the urinary system and male genital organs." BJU International 94(4): 675675.

Curatolo, P., S. Seri, M. Verdecchia and R. Bombardieri (2001). "Infantile spasms in tuberous sclerosis complex." Brain Dev 23(7): 502507.

Dabora, S. L., S. Jozwiak, D. N. Franz, P. S. Roberts, A. Nieto, J. Chung, Y. S. Choy, M. P. Reeve, E. Thiele, J. C. Egelhoff, J. Kasprzyk-Obara, D. Domanska-Pakiela and D. J. Kwiatkowski (2001). "Mutational analysis in a cohort of 224 tuberous sclerosis patients indicates increased severity of TSC2, compared with TSC1, disease in multiple organs." Am J Hum Genet 68(1): 64-80.

Davidson, M., H. Yoshidome, E. Stenroos and W. G. Johnson (1991). "Neuron-like cells in culture of tuberous sclerosis tissue." Ann N Y Acad Sci 615: 196-210.

Eble, J. N. (1998). "Angiomyolipoma of kidney." Semin Diagn Pathol 15(1): 21-40.

Fernandez-Flores, A. (2011). "Perivascular migration: a clue to the histogenesis of PEComas?" Am J Dermatopathol 33(5): 528-529.

Folpe, A. L. and D. J. Kwiatkowski (2010). "Perivascular epithelioid cell neoplasms: pathology and pathogenesis." Hum Pathol $41(1): 1-$ 15.

Franz, D. N., J. J. Bissler and F. X. McCormack (2010). "Tuberous sclerosis complex: neurological, renal and pulmonary manifestations." Neuropediatrics 41(5): 199-208.

Garami, A., F. J. T. Zwartkruis, T. Nobukuni, M. Joaquin, M. Roccio, H. Stocker, S. C. Kozma, E. Hafen, J. L. Bos and G. Thomas (2003) "Insulin Activation of Rheb, a Mediator of mTOR/S6K/4E-BP Signaling, Is Inhibited by TSC1 and 2." Molecular Cell 11(6): 1457-1466.

Gomez, M., J. Sampson and V. Holtes-Whittemore (1999). "Tuberous Sclerosis Complex." Oxford University Press 3rd edition.

Goncharova, E., D. Goncharov, D. Noonan and V. P. Krymskaya (2004). "TSC2 modulates actin cytoskeleton and focal adhesion through TSC1-binding domain and the Rac1 GTPase." J Cell Biol 167(6): 1171-1182.

Goodman, M., S. H. Lamm, A. Engel, C. W. Shepherd, O. W. Houser and M. R. Gomez (1997). "Cortical tuber count: a biomarker indicating neurologic severity of tuberous sclerosis complex." J Child Neurol 12(2): 85-90.

Grajkowska, W., K. Kotulska, E. Jurkiewicz and E. Matyja (2010). "Brain lesions in tuberous sclerosis complex. Review." Folia Neuropathol 48(3): 139-149.

Hancock, E. and J. Osborne (1998). "Treatment of infantile spasms with high-dose oral prednisolone." Dev Med Child Neurol 40(7): 500.

Hasbani, D. M. and P. B. Crino (2018). "Tuberous sclerosis complex." Handb Clin Neurol 148: 813-822.

Hayashi, T., T. Kumasaka, K. Mitani, T. Yao, K. Suda and K. Seyama (2010). "Loss of heterozygosity on tuberous sclerosis complex genes in multifocal micronodular pneumocyte hyperplasia." Mod Pathol 23(9): 1251-1260.

Holmes, G. L., C. E. Stafstrom and G. Tuberous Sclerosis Study (2007). "Tuberous sclerosis complex and epilepsy: recent developments and future challenges." Epilepsia 48(4): 617-630.

Hoogeveen-Westerveld, M., L. van Unen, A. van den Ouweland, D. Halley, A. Hoogeveen and M. Nellist (2012). "The TSC1-TSC2 complex consists of multiple TSC1 and TSC2 subunits." BMC Biochem 13: 18.

Hosoyaa, M., H. Naitoa and K. Nihei (1999). "Neurological prognosis correlated with variations over time in the number of subependymal nodules in tuberous sclerosis." Brain \& Development 21(8): 4.

Houser, O. W. and M. R. Gomez (1992). "CT and MR imaging of intracranial tuberous sclerosis." J Dermatol 19(11): 904-908.

Humphrey, A., J. Williams, E. Pinto and P. F. Bolton (2004). "A prospective longitudinal study of early cognitive development in tuberous sclerosis - a clinic based study." Eur Child Adolesc Psychiatry 13(3): 159-165. 
Hung, C. C., Y. N. Su, S. C. Chien, H. H. Liou, C. C. Chen, P. C. Chen, C. J. Hsieh, C. P. Chen, W. T. Lee, W. L. Lin and C. N. Lee (2006). "Molecular and clinical analyses of 84 patients with tuberous sclerosis complex." BMC Med Genet 7: 72.

Huttenlocher, P. R. and R. L. Wollmann (1991). "Cellular neuropathology of tuberous sclerosis." Ann N Y Acad Sci 615: 140-148.

Inoki, K., Y. Li, T. Xu and K. L. Guan (2003). "Rheb GTPase is a direct target of TSC2 GAP activity and regulates mTOR signaling." Genes Dev 17(15): 1829-1834

Inoue, H., T. Uyama, T. Suzuki, M. Kazami, O. Hino, T. Kobayashi, K. Kobayashi, T. Tadokoro and Y. Yamamoto (2010). "Phosphorylated hamartin-Hsp70 complex regulates apoptosis via mitochondrial localization." Biochem Biophys Res Commun 391(1): 1148-1153.

Jansen, F. E., O. van Nieuwenhuizen and A. C. van Huffelen (2004). "Tuberous sclerosis complex and its founders." J Neurol Neurosurg Psychiatry 75(5): 770

Johnson, W. G., H. Yoshidome, E. S. Stenroos and M. M. Davidson (1991). "Origin of the neuron-like cells in tuberous sclerosis tissues." Ann N Y Acad Sci 615: 211-219.

Joinson, C., F. J. O'Callaghan, J. P. Osborne, C. Martyn, T. Harris and P. F. Bolton (2003). "Learning disability and epilepsy in an epidemiological sample of individuals with tuberous sclerosis complex." Psychol Med 33(2): 335-344.

Jones, A. C., M. M. Shyamsundar, M. W. Thomas, J. Maynard, S. Idziaszczyk, S. Tomkins, J. R. Sampson and J. P. Cheadle (1999). "Comprehensive mutation analysis of TSC1 and TSC2-and phenotypic correlations in 150 families with tuberous sclerosis." Am J Hum Genet 64(5): 1305-1315.

Jozwiak, J., S. Jozwiak and P. Skopinski (2005). "Immunohistochemical and microscopic studies on giant cells in tuberous sclerosis." Histol Histopathol 20(4): 1321-1326.

Knudson, A. G., Jr. (1971). "Mutation and cancer: statistical study of retinoblastoma." Proc Natl Acad Sci U S A 68(4): 820-823.

Kozlowski, P., P. Roberts, S. Dabora, D. Franz, J. Bissler, H. Northrup, K. S. Au, R. Lazarus, D. Domanska-Pakiela, K. Kotulska, S. Jozwiak and D. J. Kwiatkowski (2007). "Identification of 54 large deletions/duplications in TSC1 and TSC2 using MLPA, and genotype-phenotype correlations." Hum Genet 121(3-4): 389-400.

Krueger, D. A., H. Northrup and G. International Tuberous Sclerosis Complex Consensus (2013). "Tuberous sclerosis complex surveillance and management: recommendations of the 2012 International Tuberous Sclerosis Complex Consensus Conference." Pediatr Neurol 49(4): 255-265.

Kwiatkowski, D. J. (1994). "Tuberous Sclerosis." Archives of Dermatology 130(3).

Lamb, R. F., C. Roy, T. J. Diefenbach, H. V. Vinters, M. W. Johnson, D. G. Jay and A. Hall (2000). "The TSC1 tumour suppressor hamartin regulates cell adhesion through ERM proteins and the GTPase Rho." Nat Cell Biol 2(5): 281-287.

Lotz, G. P., H. Lin, A. Harst and W. M. Obermann (2003). "Aha1 binds to the middle domain of Hsp90, contributes to client protein activation, and stimulates the ATPase activity of the molecular chaperone." J Biol Chem 278(19): 17228-17235.

Maheshwar, M. M., J. P. Cheadle, A. C. Jones, J. Myring, A. E. Fryer, P. C. Harris and J. R. Sampson (1997). "The GAP-Related Domain of Tuberin, the Product of the TSC2 Gene, is a Target for Missense Mutations in Tuberous Sclerosis." Human Molecular Genetics 6(11): 1991-1996.

Martignoni, G., M. Pea, D. Reghellin, G. Zamboni and F. Bonetti (2008). "PEComas: the past, the present and the future." Virchows Arch 452(2): 119-132.

Maruyama, H., K. Seyama, J. Sobajima, K. Kitamura, T. Sobajima, T. Fukuda, K. Hamada, M. Tsutsumi, O. Hino and Y. Konishi (2001). "Multifocal micronodular pneumocyte hyperplasia and lymphangioleiomyomatosis in tuberous sclerosis with a TSC2 gene." Mod Pathol 14(6): 609-614.

McCall, T., S. S. Chin, K. L. Salzman and D. W. Fults (2006). "Tuberous sclerosis: a syndrome of incomplete tumor suppression." Neurosurg Focus 20(1): E3.

Mizuguchi, M. and S. Takashima (2001). "Neuropathology of tuberous sclerosis." Brain Dev 23(7): 508-515. 
Moss, J., N. A. Avila, P. M. Barnes, R. A. Litzenberger, J. Bechtle, P. G. Brooks, C. J. Hedin, S. Hunsberger and A. S. Kristof (2001). "Prevalence and clinical characteristics of lymphangioleiomyomatosis (LAM) in patients with tuberous sclerosis complex." Am J Respir Crit Care Med 164(4): 669-671.

Muir, T. E., K. O. Leslie, H. Popper, M. Kitaichi, E. Gagné, J. K. Emelin, H. V. Vinters and T. V. Colby (1998). "Micronodular pneumocyte hyperplasia." Am J Surg Pathol 22(4): 8.

Nellist, M., D. van den Heuvel, D. Schluep, C. Exalto, M. Goedbloed, A. Maat-Kievit, T. van Essen, K. van Spaendonck-Zwarts, F. Jansen, P. Helderman, G. Bartalini, O. Vierimaa, M. Penttinen, J. van den Ende, A. van den Ouweland and D. Halley (2009). "Missense mutations to the TSC1 gene cause tuberous sclerosis complex." Eur J Hum Genet 17(3): 319-328.

Nellist, M., M. A. van Slegtenhorst, M. Goedbloed, A. M. van den Ouweland, D. J. Halley and P. van der Sluijs (1999). "Characterization of the cytosolic tuberin-hamartin complex. Tuberin is a cytosolic chaperone for hamartin." J Biol Chem 274(50): 35647-35652.

Niida, Y., N. Lawrence-Smith, A. Banwell, E. Hammer, J. Lewis, R. L. Beauchamp, K. Sims, V. Ramesh and L. Ozelius (1999). "Analysis of bothTSC1 andTSC2 for germline mutations in 126 unrelated patients with tuberous sclerosis." Human Mutation 14(5): 412-422.

Niida, Y., A. O. Stemmer-Rachamimov, M. Logrip, D. Tapon, R. Perez, D. J. Kwiatkowski, K. Sims, M. MacCollin, D. N. Louis and V. Ramesh (2001). "Survey of somatic mutations in tuberous sclerosis complex (TSC) hamartomas suggests different genetic mechanisms for pathogenesis of TSC lesions." Am J Hum Genet 69(3): 493-503.

Nishio, S., T. Morioka, S. Suzuki, R. Kira, F. Mihara and M. Fukui (2001). "Subependymal giant cell astrocytoma: clinical and neuroimaging features of four cases." J Clin Neurosci 8(1): 31-34.

Northrup, H., D. A. Krueger and G. International Tuberous Sclerosis Complex Consensus (2013). "Tuberous sclerosis complex diagnostic criteria update: recommendations of the 2012 linternational Tuberous Sclerosis Complex Consensus Conference." Pediatr Neurol 49(4): 243-254.

Northrup, H., D. A. Krueger and G. International Tuberous Sclerosis Complex Consensus (2013). "Tuberous sclerosis complex diagnostic criteria update: recommendations of the 2012 international tuberous sclerosis complex consensus conference." Pediatr Neurol 49(4): 243-254.

Northrup, H., J. W. Wheless, T. K. Bertin and R. A. Lewis (1993). "Variability of expression in tuberous sclerosis." J Med Genet 30(1): 4143.

O'Callaghan, F. J., A. W. Shiell, J. P. Osborne and C. N. Martyn (1998). "Prevalence of tuberous sclerosis estimated by capture-recapture analysis." Lancet 351(9114): 1490.

Orlova, K. A. and P. B. Crino (2010). "The tuberous sclerosis complex." Ann N Y Acad Sci 1184: 87-105.

Panaretou, B., C. Prodromou, S. M. Roe, R. O'Brien, J. E. Ladbury, P. W. Piper and L. H. Pearl (1998). "ATP binding and hydrolysis are essential to the function of the Hsp90 molecular chaperone in vivo." EMBO J 17(16): 4829-4836.

Panaretou, B., G. Siligardi, P. Meyer, A. Maloney, J. K. Sullivan, S. Singh, S. H. Millson, P. A. Clarke, S. Naaby-Hansen, R. Stein, R. Cramer M. Mollapour, P. Workman, P. W. Piper, L. H. Pearl and C. Prodromou (2002). "Activation of the ATPase activity of hsp90 by the stressregulated cochaperone aha1." Mol Cell 10(6): 1307-1318.

Plank, T. L., R. S. Yeung and E. P. Henske (1998). "Hamartin, the product of the tuberous sclerosis 1 (TSC1) gene, interacts with tuberin and appears to be localized to cytoplasmic vesicles." Cancer Research 58(21): 4766-4770.

Prabhakar, S., X. Zhang, J. Goto, S. Han, C. Lai, R. Bronson, M. Sena-Esteves, V. Ramesh, A. Stemmer-Rachamimov, D. J. Kwiatkowski and X. O. Breakefield (2015). "Survival benefit and phenotypic improvement by hamartin gene therapy in a tuberous sclerosis mouse brain model." Neurobiol Dis 82: 22-31.

Prodromou, C. and L. H. Pearl (2003). "Structure and functional relationships of Hsp90." Curr Cancer Drug Targets 3(5): 301-323.

Rakowski, S. K., E. B. Winterkorn, E. Paul, D. J. Steele, E. F. Halpern and E. A. Thiele (2006). "Renal manifestations of tuberous sclerosis complex: Incidence, prognosis, and predictive factors." Kidney Int 70(10): 1777-1782. 
Rendtorff, N. D., B. Bjerregaard, M. Frodin, S. Kjaergaard, H. Hove, F. Skovby, K. Brondum-Nielsen, M. Schwartz and G. Danish Tuberous Sclerosis (2005). "Analysis of 65 tuberous sclerosis complex (TSC) patients by TSC2 DGGE, TSC1/TSC2 MLPA, and TSC1 long-range PCR sequencing, and report of 28 novel mutations." Hum Mutat 26(4): 374-383.

Roach, E. S., M. R. Gomez and H. Northrup (1998). "Tuberous sclerosis complex consensus conference: revised clinical diagnostic criteria." J Child Neurol 13(12): 624-628.

Roach, E. S. and S. P. Sparagana (2004). "Diagnosis of tuberous sclerosis complex." J Child Neurol 19(9): 643-649.

Sampson, J. R., S. J. Scahill, J. B. Stephenson, L. Mann and J. M. Connor (1989). "Genetic aspects of tuberous sclerosis in the west of Scotland." J Med Genet 26(1): 28-31.

Sancak, O., M. Nellist, M. Goedbloed, P. Elfferich, C. Wouters, A. Maat-Kievit, B. Zonnenberg, S. Verhoef, D. Halley and A. van den Ouweland (2005). "Mutational analysis of the TSC1 and TSC2 genes in a diagnostic setting: genotype--phenotype correlations and comparison of diagnostic DNA techniques in Tuberous Sclerosis Complex." Eur J Hum Genet 13(6): 731-741.

Sasongko, T. H., M. Wataya-Kaneda, K. Koterazawa, Gunadi, S. Yusoff, I. S. Harahap, M. J. Lee, M. Matsuo and H. Nishio (2008). "Novel mutations in 21 patients with tuberous sclerosis complex and variation of tandem splice-acceptor sites in TSC1 exon 14." Kobe J Med Sci 54(1): E73-81.

Scheithauer, B. J. and T. J. Reagan (1999). "In: Gomez, M. R. e Whittemore, V. H. 3a Ed. Tuberous Sclerois Complex." Neurophatology: 44.

Schubert, U., L. C. Anton, J. Gibbs, C. C. Norbury, J. W. Yewdell and J. R. Bennink (2000). "Rapid degradation of a large fraction of newly synthesized proteins by proteasomes." Nature 404(6779): 770-774.

Shepherd, C. W., O. W. Houser and M. R. Gomez (1995). "MR findings in tuberous sclerosis complex and correlation with seizure development and mental impairment." AJNR Am J Neuroradiol 16(1): 149-155.

Shepherd, C. W., B. W. Scheithauer, M. R. Gomez, H. J. Altermatt and J. A. Katzmann (1991). "Subependymal giant cell astrocytoma: a clinical, pathological, and flow cytometric study." Neurosurgery 28(6): 864-868.

Smolarek, T. A., L. L. Wessner, F. X. McCormack, J. C. Mylet, A. G. Menon and E. P. Henske (1998). "Evidence that lymphangiomyomatosis is caused by TSC2 mutations: chromosome 16p13 loss of heterozygosity in angiomyolipomas and lymph nodes from women with lymphangiomyomatosis." Am J Hum Genet 62(4): 810-815

Stefansson, K. (1991). "Tuberous sclerosis." Mayo Clin Proc 66(8): 868-872.

Tee, A. R., D. C. Fingar, B. D. Manning, D. J. Kwiatkowski, L. C. Cantley and J. Blenis (2002). "Tuberous sclerosis complex-1 and -2 gene products function together to inhibit mammalian target of rapamycin (mTOR)-mediated downstream signaling." Proc Natl Acad Sci U S A 99(21): 13571-13576

Tee, A. R., B. D. Manning, P. P. Roux, L. C. Cantley and J. Blenis (2003). "Tuberous Sclerosis Complex Gene Products, Tuberin and Hamartin, Control mTOR Signaling by Acting as a GTPase-Activating Protein Complex toward Rheb." Current Biology 13(15): 1259-1268.

Telfeian, A. E., A. Judkins, D. Younkin, A. N. Pollock and P. Crino (2004). "Subependymal giant cell astrocytoma with cranial and spinal metastases in a patient with tuberous sclerosis. Case report." J Neurosurg 100(5 Suppl Pediatrics): 498-500.

Thiele, E. A. (2004). "Managing epilepsy in tuberous sclerosis complex." J Child Neurol 19(9): 680-686.

Trombley, I. K. and S. S. Mirra (1981). "Ultrastructure of tuberous sclerosis: cortical tuber and subependymal tumor." Ann Neurol 9(2): 174-181.

van Eeghen, A. M., L. O. Teran, J. Johnson, M. B. Pulsifer, E. A. Thiele and P. Caruso (2013). "The neuroanatomical phenotype of tuberous sclerosis complex: focus on radial migration lines." Neuroradiology 55(8): 1007-1014.

van Slegtenhorst, M., R. de Hoogt, C. Hermans, M. Nellist, B. Janssen, S. Verhoef, D. Lindhout, A. van den Ouweland, D. Halley, J. Young, M. Burley, S. Jeremiah, K. Woodward, J. Nahmias, M. Fox, R. Ekong, J. Osborne, J. Wolfe, S. Povey, R. G. Snell, J. P. Cheadle, A. C. Jones, M. Tachataki, D. Ravine, J. R. Sampson, M. P. Reeve, P. Richardson, F. Wilmer, C. Munro, T. L. Hawkins, T. Sepp, J. B. Ali, S. Ward, A. J. Green, J. R. Yates, J. Kwiatkowska, E. P. Henske, M. P. Short, J. H. Haines, S. Jozwiak and D. J. Kwiatkowski (1997). "Identification of the tuberous sclerosis gene TSC1 on chromosome 9q34." Science 277(5327): 805-808. 
van Slegtenhorst, M., M. Nellist, B. Nagelkerken, J. Cheadle, R. Snell, A. van den Ouweland, A. Reuser, J. Sampson, D. Halley and P. van der Sluijs (1998). "Interaction between hamartin and tuberin, the TSC1 and TSC2 gene products." Human molecular genetics 7(6): 10531057

van Slegtenhorst, M. A., A. Verhoef, A. Tempelaars, L. Bakker, Q. Wang, M. Wessels, R. Bakker, M. Nellist, D. Lindhout, D. Halley and A. van den Ouweland (1999). "Mutational spectrum of the TSC1 gene in a cohort of 225 tuberous sclerosis complex patients: no evidence for genotype-phenotype correlation." J Med Genet 36(4): 5.

von Ranke, F. M., I. M. Faria, G. Zanetti, B. Hochhegger, A. S. Souza, Jr. and E. Marchiori (2017). "Imaging of tuberous sclerosis complex: a pictorial review." Radiol Bras 50(1): 48-54.

Webb, D. W. and J. P. Osborne (1991). "Non-penetrance in tuberous sclerosis." J Med Genet 28(6): 417-419.

Weeks, D. A., R. L. Malott, M. Arnesen, C. Zuppan, D. Aitken and G. Mierau (1991). "Hepatic angiomyolipoma with striated granules and positivity with melanoma--specific antibody (HMB-45): a report of two cases." Ultrastruct Pathol 15(4-5): 563-571.

Wilson, P. J., V. Ramesh, A. Kristiansen, C. Bove, S. Jozwiak, D. J. Kwiatkowski, M. P. Short and J. L. Haines (1996). "Novel Mutations Detected in the TSC2 Hene From Both Sporadic and Familial TSC Patients." Human Molecular Genetics 5(2): 249-256.

Woodford, M. R., D. M. Dunn, A. R. Blanden, D. Capriotti, D. Loiselle, C. Prodromou, B. Panaretou, P. F. Hughes, A. Smith, W. Ackerman, T. A. Haystead, S. N. Loh, D. Bourboulia, L. S. Schmidt, W. Marston Linehan, G. Bratslavsky and M. Mollapour (2016). "The FNIP cochaperones decelerate the Hsp90 chaperone cycle and enhance drug binding." Nat Commun 7: 12037.

Woodford, M. R., R. A. Sager, E. Marris, D. M. Dunn, A. R. Blanden, R. L. Murphy, N. Rensing, O. Shapiro, B. Panaretou, C. Prodromou, S. N. Loh, D. H. Gutmann, D. Bourboulia, G. Bratslavsky, M. Wong and M. Mollapour (2017). "Tumor suppressor Tsc1 is a new Hsp90 cochaperone that facilitates folding of kinase and non-kinase clients." EMBO J 36(24): 3650-3665.

Yang, L., X. L. Feng, S. Shen, L. Shan, H. F. Zhang, X. Y. Liu and N. Lv (2012). "Clinicopathological analysis of 156 patients with angiomyolipoma originating from different organs." Oncol Lett 3(3): 586-590. 


\section{Chapter II}

\section{TSC1 and TSC2 pathogenic variants in patients with a clinical diagnosis of TSC}




\section{List of Acronyms}

ACMG: American College of Medical Genetics and Genomics

DMSO: Dimethyl sulfoxide

GADPH: Glyceraldehyde-3-phosphate dehydrogenase

GFI1B: Growth Factor-Independent 1B

GRAACC: Grupo de Apoio ao Adolescente e à Criança com Câncer

HC-UFPR: Hospital de Clínicas of the Universidade Federal do Paraná

LOVD: Leiden Open Variation Database

mTORC1: Mechanistic target of rapamycin kinase complex 1

MLPA: Multiplex ligation-dependent probe amplification

NGS: Next generation sequencing

panel-NGS: Next Generation Sequencing on a customized gene panel chip

NMI: No mutation identified

ORF: Open reading frame

PKD1: Polycystic Kidney Disease 1 gene

PKDTS: Polycystic Kidney Disease, Infantile Severe, with tuberous sclerosis

PCR: Polymerase chain reaction

Q-PCR: Quantitative PCR

RHEB: Ras homologue enriched in brain

S6K1: S6 kinase 1

HSCM-SP: Santa Casa de Misericórdia

SFs: Secondary findings discoveries

SINE: Short interspersed nuclear element

SNV: Single nucleotide variants

TSC: Tuberous sclerosis complex

VUS: Variant of unclear significance

WT: Wild-type 


\section{List of Tables}

Table II-1: TSC1 and TSC2 pathogenic variant classification and worldwide distribution . .44

Table II-2: Monoclonal and polyclonal antibodies used to functional assessment of TSC2 DNA variants. .......52

Table II-3: TSC1 and TSC2 pathogenic DNA variants according to hospital of patient origin. .54

Table II-4: TSC1 pathogenic DNA variants in a cohort of 117 TSC patients...............................................58

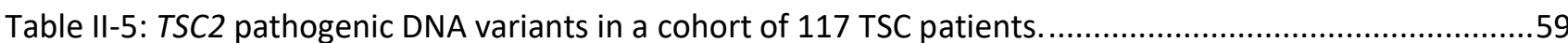

Table II-6: Pathogenicity criteria for classification of TSC2 missense ( $N=20)$ and in-frame deletion (N=5) DNA variants with low population frequency (Richards, Aziz et al. 2015) ............................................66

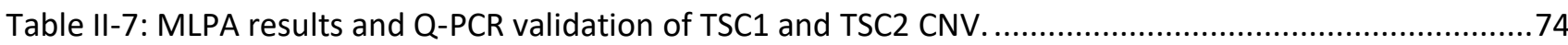

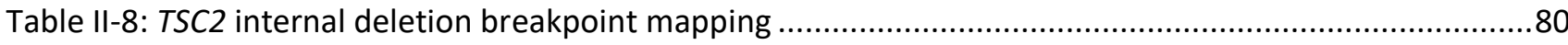




\section{List of Figures}

_Toc16892673

Figure II-1: Workflow of in silico and in vivo analysis of DNA variants detected on TSC1 and TSC2. .50

Figure II-2: TSC2 missense and in-frame deletion functional assessment workflow. BDT: Big Dye terminator reaction .52

Figure II-3: Overview of the distribution of TSC1 and TSC2 pathogenic DNA variants according to frequency and alteration type. (A) Ratio of TSC1 and TSC2 pathogenic variants .55

Figure II-4: Schematic representation of TSC1 protein segments and distribution of all TSC1 pathogenic variants according to the amino acid number. .56

Figure II-5: Schematic representation of TSC2 protein segments and distribution of all TSC2 pathogenic variants according to the amino acid number. Splicing variants are not represented. .57

Figure II-6: Workflow of the pathogenicity classification of 20 TSC2 missense DNA variants .64

Figure II-7: Functional assessment of the TSC2 (NM_000548.3) variants. .69

Figure II-8: Schematic representation of distribution of pathogenic TSC2 splice variants. .70

Figure II-9: Functional evaluation of a substitution DNA variant in the TSC2 gene (c.4493G>T p.Ser1498Ile variant). .71

Figure II-10: Functional evaluation of a deep intronic DNA variation in the TSC2 gene (c.1361+54_1361+57del variant). .73

Figure II-11: MPLA and Q-PCR results for TSC1 or TSC2 deletions and duplication. Left panel is MLPA report and right panel the Q-PCR results .75

Figure II-12: Breakpoint mapping of the TSC2 intragenic deletion c.(975+_976-)_(1716+_1717-)del. .81

Figure II-13: Schematic representation of TSC1 and the segmental deletion c.-234-?_-144+?del of exon 1 on patient\#25 c.(737+1_738-1)_(*1+_?)del from exon 9 to exon 23 fond on patient\#29.

Figure II-14: Schematic representation of TSC2 and the segmental deletion c.(975+628)_(1716+41)del from exon 11 to exon 16, c.(5068+1_5069-1)_(*102_?)del from exon 39 to exon 42, c.(1599+1_16001)_(2545+1_2546-1)del from exon 16 to exon 22, c.(?_-30)_(*102_?)del whole TSC2, c.(1716+1_17171)_(2545+1_2546-1)del from exon 17 to exon 22, c.(774+1_775-1)_(848+1_849-1)del from exon 22 to exon 42, and duplication c.(2355+1_2356-1)_(*102_?)dup form exon 22 to 42 . .84

Figure II-15: Global alignment of TSC2, phosphorylation site of GAP Domain and positioning of TSC2:p.Tyr1608del, p.lle1614del and p.Arg1743Gly variants on predicted Homo sapiens GAP domain structure (PDB: 1SRQ). .89

Figure II-16: Global alignment of TSC2, phosphorylation site of TSC1 binding domain and positioning of TSC2:p.Trp167Arg variant on predicted Chaetomium thermophilum TSC1 binding domain structure (PDB:5HIU) 
Figure II-17: Breakpoint mapping of the TSC2 intragenic deletion c.(975+_976-)_(1716+_1717-)del . (A) Sanger sequencing of the DNA from the patient with the intragenic TSC2 deletion c.(975+_976-

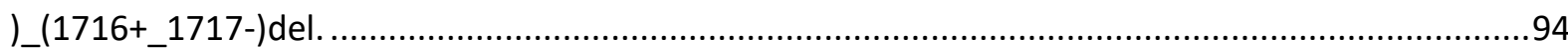




\section{Summary}

II. Chapter II

I. Introduction

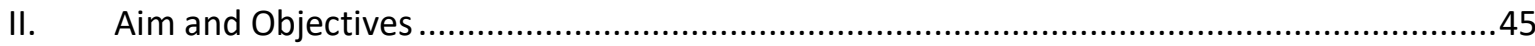
A. Aim
.45
B. Objectives 45

III. Subjects and Methods .46
A. Patients .46
B. Sanger Sequencing and Next Generation Sequencing .46
C. Multiplex ligation-dependent probe amplification (MLPA) .48
D. Quantitative polymerase chain reaction .48
E. Pipeline of in silico and in vivo analysis of DNA variants .48
F. Functional assessment of TSC2 VUS missense and in-frame deletion .50
G. Splicing effect of TSC2 missense and deep intronic variants.

IV. Results

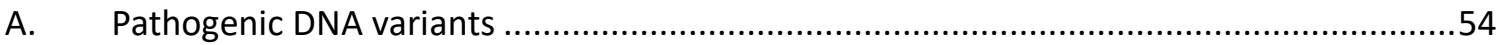

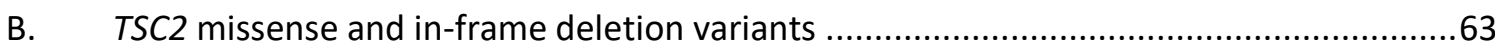

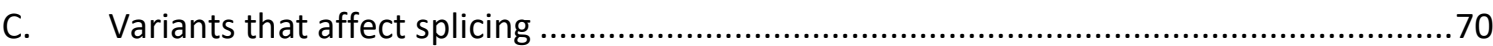

D. Large segmental gene deletions and duplications ......................................... 73

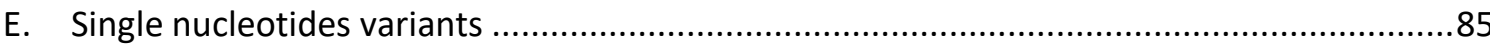

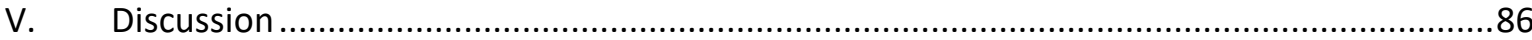

A. TSC2 missense and in-frame deletion effect on mTOR .........................................87

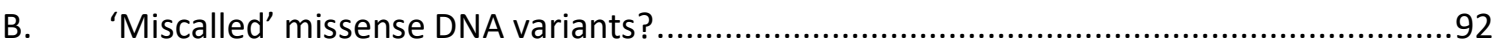

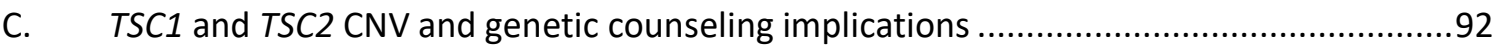

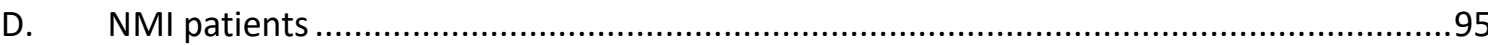

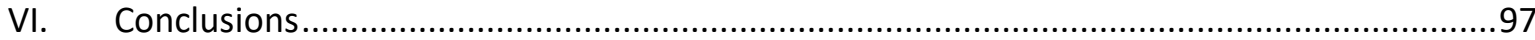

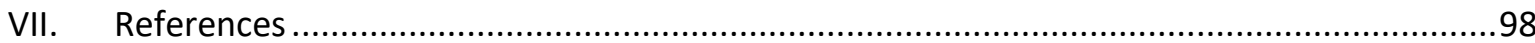

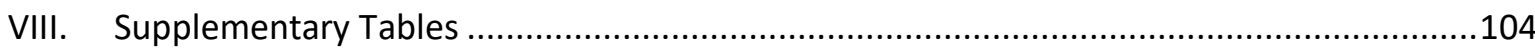




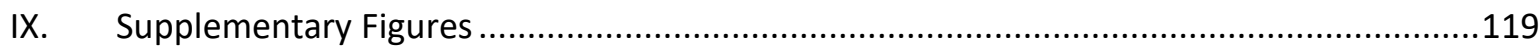




\section{Introduction}

Tuberous sclerosis complex (TSC) is a genetic disease affecting 1 in every 6,000 to 10,000 live-born infants, showing no ethnic bias. It is characterized by hamartoma growth more often in the brain, skin, kidneys, heart, lungs, and retina (Sampson, Scahill et al. 1989, O'Callaghan, Shiell et al. 1998, Au, Williams et al. 2007, Northrup, Krueger et al. 2013). Renal manifestations, epilepsy and intellectual disability are important sources of morbidity (Rakowski, Winterkorn et al. 2006, Moavero, Pinci et al. 2011, Northrup, Krueger et al. 2013, Kwiatkowski, Palmer et al. 2015). Northrup et al (2013) presented an up-to-date classification of TSC lesions considered for the clinical diagnosis of the disease.

TSC is an autosomal dominant disorder due to pathogenic variants in either TSC1 or TSC2 tumour suppressor genes (Consortium 1993, van Slegtenhorst, de Hoogt et al. 1997). The TSC1 gene (NG_012386.1, Ch:9q34.1, MIM\#605284), with approximately $55 \mathrm{~kb}$ comprising 23 exons, codes for the TSC1 protein (hamartin, NP_000359.1) with 130 kDa (van Slegtenhorst, de Hoogt et al. 1997). The TSC2 (NG_005895.1, Ch:16p13.3, MIM\#191092) gene has 42 exons and nearly $40 \mathrm{~kb}$, and encodes the TSC2 protein (tuberin, NP_000539.2) with 180 kDa (Consortium 1993, van Slegtenhorst, de Hoogt et al. 1997, Jones, Shyamsundar et al. 1999, Dabora, Jozwiak et al. 2001, Sancak, Nellist et al. 2005, Au, Williams et al. 2007). TSC1 and TSC2 physically interact as a cytosolic heterodimer (TSC1/2 complex), which acts as mediator of intracellular signals to inhibit the mechanistic target of rapamycin (mTOR) kinase complex 1 (mTORC1) through the small GTPase RHEB (Ras homologue enriched in brain; (Garami, Zwartkruis et al. 2003, Tee, Manning et al. 2003). Inactivation of the TSC1/2 complex results in increased levels of RHEB-GTP, required for activation of mTORC1, which phosphorylates downstream targets. Inactivation of the TSC1/2 complex increases mTORC1 phosphorylation of, for instance, the 56 kinase 1 (S6K1) at residue $\mathrm{Thr}^{389}$ (Goncharova, Goncharov et al. 2002, Tee, Fingar et al. 2002) causing excessive cell growth and augmented cell proliferation.

Gómez (1979) first established a broad set of major and minor clinical criteria for the definite, probable or possible diagnosis of TSC. Since then TSC diagnosis criteria have been reviewed and updated (Roach, Gomez et al. 1998, Northrup, Krueger et al. 2013). Besides updating the TSC clinical criteria, the 2012 working group implemented the genetic diagnostic criterion, by which the detection of a pathogenic variant in ether TSC1 or TSC2 genes is sufficient for the definite molecular diagnosis of TSC (Northrup, Krueger et al. 2013), following the American College of Medical Genetics and Genomics (ACMG) guidelines for pathogenicity report of DNA variants (Richards, Aziz et al. 2015). Nearly 90\% of patients with definite clinical diagnosis of TSC have a TSC1 or TSC2 phatogenic DNA variant identified by genetic testing (Peron, Au et al. 2018). The ratio between TSC1 and TSC2 pathogenic variant frequencies appears close to 1:1 among TSC familial cases, and may vary from 1:3 to 1:6 among de novo cases (Jones, Shyamsundar et al. 1999, Dabora, Jozwiak et al. 2001, Rendtorff, Bjerregaard et al. 2005, Au, Williams et al. 2007). De novo TSC cases with more severe angiomyolipomas 
and/or intellectual disability tend to have germline mutations more frequently on the TSC2 gene than the TSC1 gene (Dabora, Jozwiak et al. 2001, Sancak, Nellist et al. 2005, Au, Williams et al. 2007). In approximately 10\% of patients with definite clinical diagnosis of TSC, no pathogenic DNA variant is identified in TSC1 or TSC2 by current molecular techniques (Kozlowski, Roberts et al. 2007, Camposano, Greenberg et al. 2009, Qin, Kozlowski et al. 2010, Tyburczy, Wang et al. 2013, Nellist, Brouwer et al. 2015). TSC1 or TSC2 somatic mosaicism and deep intronic DNA variants have recently been reported in those patients with no mutation identified (NMI) in the coding region of either gene (Nellist, Brouwer et al. 2015, Tyburczy, Dies et al. 2015). Worldwide studies detected pathogenic DNA variants on TSC1 and TSC2 by gold-standard methodologies, such as Sanger sequencing and MLPA, as well as next-generation sequencing (NGS), disclosing similar results (Table II-1).

The detection of a pathogenic DNA variant in the TSC1 or TSC2 genes as an independent diagnostic criterion rendered it sufficient for the diagnosis or prediction of TSC regardless of the clinical findings (Northrup, Krueger et al. 2013). It allows in some cases for early diagnosis notably in young individuals who may not yet fulfil the criteria for a definite clinical diagnosis. Although health economics studies are needed to evaluate the cost-effectiveness of NGS testing in TSC patients that present with isolate lesions, it is evident that gene panel NGS costs have been declining over the years (Schwarze, Buchanan et al. 2018). Genetic testbased early TSC diagnosis is likely to accelerate implementation of clinical surveillance and selection of patients for treatments that will potentially lead to better clinical outcomes.

In a previous work, we have evaluated by Sanger sequencing the TSC1 coding sequence in a cohort of 28 TSC patients (Dufner-Almeida 2014). In the present study, we extended the cohort to a total of 117 patients from the states of São Paulo and Paraná, Brazil, and employed their leukocyte DNA to search for TSC1 and TSC2 germline pathogenic DNA variants and segmental deletions/duplications. We disclosed a pathogenic variant detection rate of $87,18 \%$ (102/117), with 84 (82.35\%) pathogenic variants in the TSC2 and 18 (17.64\%) in the TSC1. 
Table II-1: TSC1 and TSC2 pathogenic variant classification and worldwide distribution.

\begin{tabular}{|c|c|c|c|c|c|c|c|c|c|c|c|c|c|c|c|c|c|c|}
\hline \multirow[b]{2}{*}{ Reference } & \multirow[b]{2}{*}{ Country } & \multirow[b]{2}{*}{$\begin{array}{l}\text { Patient } \\
\text { s }\end{array}$} & \multirow[b]{2}{*}{$\begin{array}{l}\text { Pathogenic } \\
\text { Variants }\end{array}$} & \multirow[b]{2}{*}{$\begin{array}{l}\text { TSC1/TSC2 } \\
\text { ratio }\end{array}$} & \multicolumn{7}{|c|}{ TSC1 } & \multicolumn{7}{|c|}{ TSC2 } \\
\hline & & & & & Total & 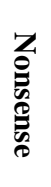 & 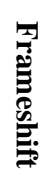 & 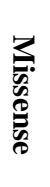 & 葛. & 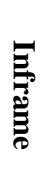 & 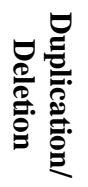 & Total & 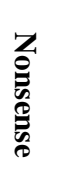 & 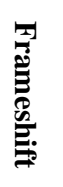 & 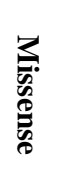 & 番 & 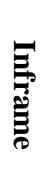 & 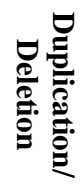 \\
\hline Jones, A. C. et. al. (1997) * & UK & 171 & $21(12.3 \%)$ & - & $21(100 \%)$ & 7 & 12 & 1 & 2 & - & - & - & - & - & - & - & - & - \\
\hline Ali, J. B., et. al. (1998) * & UK & 83 & $16(19.3 \%)$ & - & $16(100 \%)$ & 6 & 8 & - & 2 & - & - & - & - & - & - & - & - & - \\
\hline $\begin{array}{l}\text { Au, K. S., et. al. (1998) } * * \\
\text { Beauchamp, R. L. et. al. (1998) }\end{array}$ & USA & 90 & $22(24.4 \%)$ & - & - & - & - & - & - & - & - & $22(100 \%)$ & 2 & 8 & 10 & - & 2 & - \\
\hline & USA & 40 & $21(52.5 \%)$ & - & - & - & - & - & - & - & - & $21(100 \%)$ & 6 & 5 & 6 & 3 & 1 & - \\
\hline Young, J. M. et. al. (1998) * & UK & 79 & $27(34.2 \%)$ & - & $27(100 \%)$ & 13 & 13 & - & 1 & - & - & - & - & - & - & - & - & - \\
\hline Jones, A. C. et. al. (1999) & UK & 150 & $120(80 \%)$ & $1: 4.4$ & $22(18.3 \%)$ & 8 & 12 & 1 & 2 & - & - & $\begin{array}{c}98 \\
(81.7 \%)\end{array}$ & 20 & 21 & 22 & 8 & 5 & 22 \\
\hline Niida, Y. et. al. (1999) & USA & 126 & $74(59 \%)$ & $1: 3.6$ & $16(21.6 \%)$ & 7 & 7 & - & 2 & - & - & $\begin{array}{c}58 \\
(78.4 \%)\end{array}$ & 14 & 21 & 13 & 4 & 2 & 4 \\
\hline van Slegtenhorst, M. A. (1999) * & $\begin{array}{l}\text { The } \\
\text { Netherlands }\end{array}$ & 225 & $29(12.8 \%)$ & - & $29(100 \%)$ & 11 & 17 & - & 1 & - & - & - & - & - & - & - & - & - \\
\hline Dabora, S. L. et. al. (2001) & USA & 224 & $166(83 \%)$ & $1: 4.9$ & $28(15.0 \%)$ & 11 & 15 & - & 2 & - & - & $138(85 \%)$ & 37 & 43 & 31 & 27 & - & - \\
\hline Rendtorff, N. D. et. al. (2005) & Denmark & 65 & $51(78 \%)$ & $1: 3.6$ & $11(21.6 \%)$ & 4 & 6 & - & 1 & - & - & $\begin{array}{c}40 \\
(78.4 \%)\end{array}$ & 10 & 10 & 10 & 6 & 2 & 2 \\
\hline Hung, C. C. et. al. (2006) & Taiwan & 84 & $\begin{array}{c}64(76 \%) \\
243\end{array}$ & $1: 6.1$ & $9(14 \%)$ & 5 & 3 & - & - & 1 & - & $55(86 \%)$ & 15 & 21 & 12 & 7 & - & - \\
\hline Au, K. S. et. al. (2007) & USA & 325 & $(74.8 \%)$ & $1: 3$ & $61(25 \%)$ & 21 & 35 & 1 & 4 & - & - & $182(75 \%)$ & 39 & 57 & 58 & 25 & - & 3 \\
\hline Kozlowski, P. et. al. (2007) *** & USA & 261 & $54(20.7 \%)$ & $1: 12.5$ & $4(7 \%)$ & - & - & - & - & - & 4 & $50(93 \%)$ & - & - & - & - & - & 50 \\
\hline Sasongko, T. H. et. al. (2008) & Japan & 21 & $16(76.2 \%)$ & $1.3: 1$ & $9(56.3 \%)$ & 1 & 8 & - & - & - & - & $\begin{array}{c}7(43.7 \%) \\
24\end{array}$ & - & 4 & 1 & 2 & - & - \\
\hline Chopra, M. et. al. (2011) **** & Australia & 45 & $33(73 \%)$ & $1: 2.7$ & $9(27.8 \%)$ & - & - & - & - & - & - & $\begin{array}{c}(72.2 \%) \\
20\end{array}$ & - & - & - & - & - & - \\
\hline Ni1da, Y. et. al. (2013) & Japan & 57 & $31(54.4 \%)$ & $1: 1.8$ & $11(35.5 \%)$ & 2 & 5 & 1 & 2 & - & 1 & $\begin{array}{c}(64.5 \%) \\
37\end{array}$ & - & 6 & $5^{\&}$ & 4 & 4 & 1 \\
\hline Rosset, C & Brazil & 53 & $47(88.7 \%)$ & $1: 3.4$ & $11(23.4 \%)$ & 6 & 4 & - & 1 & - & - & $(76.6 \%)$ & 12 & 7 & 2 & 8 & 3 & 5 \\
\hline
\end{tabular}

*TSC1 study only

** TSC2 study only

*** Duplication and deletion study only

**** TSC1 and TSC2 variant distribution data only

${ }^{\&}$ Including one stop codon variant 


\section{Aim and Objectives}

\section{A. Aim}

To analyze the nature, distribution and functional effects of TSC1 and TSC2 DNA variants of 100 patients with clinical definite diagnosis of TSC.

\section{B. Objectives}

1. To sequence the DNA coding sequence of $T S C 1$ and $T S C 2$ by the Sanger method or next generation sequencing (NGS);

2. To perform multiplex ligation-dependent probe amplification (MLPA) of TSC NMI patient DNA;

3. To confirm every MLPA alteration on TSCl or TSC2 by quantitative PCR (qPCR);

4. To functionally evaluate the effect of identified missense TSC1 or TSC2 DNA variants of uncertain significance on in vitro hyperactivation of mTORC1 having S6K1 $\mathrm{Thr}^{389}$ phosphorylation as reporter;

5. To functionally assess putative splicing DNA variants detected outside canonical splice sites, by overexpressing a minigene harboring the DNA variant and reporting its splicing output. 


\section{Subjects and Methods}

\section{A. Patients}

This study was approved by the Institutional Ethics Review Board from the three participating institutions (Protocol Number CAAE: 125729112.3.000.5464, Appendix 1 and 2): Santa Casa de Misericórdia (HSCM-SP), São Paulo, Brazil; Hospital de Clínicas of the Universidade Federal do Paraná (HC-UFPR), Curitiba, Brazil, and Grupo de Apoio ao Adolescente e à Criança com Câncer (GRAACC), São Paulo, Brazil. All 117 patients had informed consent signed by a parent or tutor. A definite clinical diagnosis of TSC (Northrup, Krueger et al. 2013) was the only inclusion criterion. Twenty-three patients were referred from the HSCM-SP, 75 from the HC-UFPR and 19 from GRAACC. Peripheral blood samples $(4 \mathrm{~mL})$ were harvested by venipuncture and sent to the Functional Genetics laboratory at the University of São Paulo (Instituto de Biociências, IBUSP, São Paulo, Brazil) for TSC1 and TSC2 testing. Genetic counselling has been offered to all patients after the genetic analysis had been concluded.

\section{B. Sanger Sequencing and Next Generation Sequencing}

Genomic DNA (DNA) was extracted from peripheral blood leukocytes using Puregene chemistry (QIAGEN, Hilden, Germany) and Autopure LS devices (Thermo Fisher Scientific, Waltham, Massachusetts, USA), at Centro de Estudos do Genoma Humano e Células Tronco (CEGH-CEL-IBUSP) or with QIAamp DNA Blood Midi and Maxi Kits (QIAGEN, Hilden, Germany) at the Functional Genetics laboratory, according to manufacturer recommendation. Quantification and quality control were measured on a NanoDrop 2000 Spectrophotometer (Thermo Fisher Scientific, Waltham, Massachusetts, USA) for Sanger sequencing procedures, as well as agarose gel and Qubit 2.0 (Invitrogen, Carlsbad, California, USA) for Next Generation Sequencing on a customized gene panel chip (panel-NGS).

Oligonucleotides for polymerase chain reaction (PCR) and Sanger sequencing were designed with Primer3 (v. 0.4.0 (Rozen and Skaletsky 2000) or Primer-BLAST (NCBI), with 20-22 nucleotides (optimum at 21 nt), melting temperatures of $59-61^{\circ} \mathrm{C}$ (optimum at $60^{\circ} \mathrm{C}$, and $1^{\circ} \mathrm{C}$ of maximal difference between pair oligonucleotides) and guanine and cytosine percentage of 40-60\% (optimum at 50\%). The ensemble of PCR amplicons covered all exons, intron boundaries and the core promoter regions of both TSC1 (NG_012386.1) and TSC2 (NG_005895.1) genes (Supplementary Table II-1 and Supplementary Table II-2). For TSC1, the sequenced region consisted of $\sim 9.4 \mathrm{~kb}$ of the total genomic locus ( $60 \mathrm{~kb} ; \sim 16 \%$ ), including $517 \mathrm{bp}$ of the core promoter (Gerstein, Bruce et al. 2007), and upstream sequences, $3.5 \mathrm{~kb}$ of exonic sequences and $5.4 \mathrm{~kb}$ of intronic sequences. For TSC2, the sequenced region consisted of $\sim 17.8 \mathrm{~kb}$ (46 kb; 39\%): 485 bp of the core promoter and upstream sequence, $5.6 \mathrm{~kb}$ of exonic sequences and $11.7 \mathrm{~kb}$ of intronic sequences. Coding sequence annotation was according to transcript reference sequences NM_000368.4 (TSC1) and 
NM_000548.3 (TSC2). Primer specificity was tested against the human genome build GRCh37/hg19 with the BLAT program (UCSC Genome Browser, Santa Cruz CA, USA) and nucleotide BLAST (NCBI, Bethesda MD, USA). Conditions for $25 \mu \mathrm{L}$ of PCR reaction were standardized with DNA samples from three unrelated non-TSC individuals (non-TSC): 1X PCR buffer (20 mM Tris-HCl, pH 8.4, 50 mM KCl; Invitrogen, Carlsbad, California, USA), $2 \mathrm{mM} \mathrm{MgCl}_{2}$ (Invitrogen, Carlsbad, California, USA), 5\% dimethyl sulfoxide (DMSO), each deoxyribonucleotide at $0.2 \mathrm{mM}$, primer oligonucleotide at $0.2 \mu \mathrm{M}, 80 \mathrm{ng}$ of DNA and $0.2 \mathrm{U}$ of Taq polymerase (Invitrogen, Carlsbad, California, USA). For TSC1 exon 1 plus the promoter segment, a region highly enriched in cytosines and guanines (66\%), we adopted the slowdown PCR protocol (Supplementary Table II-3, Frey, Bachmann et al. (2008)); a standard PCR protocol was employed for the remaining reactions (Supplementary Table II-4). PCR products were electrophoresed on $1.5 \%$ agarose gels with $5 \mu \mathrm{L}$ of SYBRsafe (Thermo Fisher Scientific, Waltham, Massachusetts, USA) in $50 \mathrm{~mL}$ of $90 \mathrm{mM}$ Tris and boric acid, $2 \mathrm{mM}$ EDTA (TBE), and band images were captured on a Gel Doc ${ }^{\mathrm{TM}}$ EZ System using Image Lab ${ }^{\mathrm{TM}}$ software (Bio-Rad, Hercules, California, USA). Eight microliters of PCR products were treated with $1.5 \mu \mathrm{L}$ of exonuclease I : shrimp alkaline phosphatase (5U:1U; Affymetrix, Santa Clara, California, USA) for $30-60$ minutes at $37^{\circ} \mathrm{C}$, followed by enzymatic inactivation for 15 minutes at $60^{\circ} \mathrm{C}$.

Sanger sequencing was performed on purified PCR products following the ABI BigDye terminator protocol (Applied Biosystems; Foster City, California, USA) having $4 \mu \mathrm{L}$ of enzyme-treated PCR product, the sequencing oligonucleotide primer at $0.3 \mu \mathrm{M}$ (Supplementary Table II-1 and Supplementary Table II-2), $2 \mu \mathrm{L}$ of BigDye terminator solution (v3.1), $1.5 \mu \mathrm{L}$ XX BigDye buffer (BigDyeno kit ${ }^{\circledast}$ - Applied Biosystems, Carlsbad, $\mathrm{CA})$ and $6.5 \mu \mathrm{L}$ of ultrapure water to a final reaction volume of $15 \mu \mathrm{L}$. Products were purified in a Sephadex column (Amersham Biosciences; Little Chalfont, United Kingdom), and submitted to capillary electrophoresis on an ABI 3730xI DNA Analyzer (Amersham Biosciences; Little Chalfont, United Kingdom). The results were analyzed using Sequencher V.4.10.1 or early version (Gene Codes Corporation; Ann Arbor, Michigan, USA).

Twenty-three DNA samples were submitted to NGS-panel as they had no pathogenic DNA variant identified or had been partially assessed by Sanger sequencing $(\mathrm{N}=18)$, had a missense variant with unclear significance $(\mathrm{N}=4)$ or low concentration of DNA $(\mathrm{N}=1)$. Gene probes had been designed to specifically capture the coding sequence of 95 selected genes that compose a customized gene panel. This strategy comprehends capture of coding and intronic boundary sequences of both TSC1 and TSC2 genes with Nextera rapid capture (Illumina; San Diego, California, USA). The library was quantified using Bioanalyzer 2100 (Agilent Technologies; Santa Clara, California, USA), and sequencing was performed on the MiSeq platform (Illumina; San Diego, California, USA). All reads were aligned to the human genome (build GRCh37/hg19) using the BurrowsWheeler Aligner (BWA) algorithm to efficiently align short sequencing reads against the human genome ( $\mathrm{Li}$ and Durbin 2009), followed by Genome Analysis Toolkit (GATK - McKenna, Hanna et al. (2010)) and ANNOVAR variant calling and annotation (Open Bioinformatics - Wang, Li et al. (2010)). 


\section{Multiplex ligation-dependent probe amplification (MLPA)}

The SALSA MLPA kits P124-C1 TSC1 and P046-C1 TSC2 (MRC-Holland; Amsterdam, The Netherlands) were used for detection of duplications and deletions affecting TSC1 and TSC2. (Rendtorff, Bjerregaard et al. 2005, Kozlowski, Roberts et al. 2007, Padma Priya and Dalal 2012). The three non-TSC control samples used for Sanger sequencing standardization were employed as reference for all procedures, following the manufacturer protocol. Ligated products were submitted to capillary electrophoresis on an ABI 3730xI DNA Analyzer (Amersham Biosciences; Little Chalfont, United Kingdom), and analyzed using GeneMarker (SoftGenetics; State College, Pennsylvania, USA) and Coffalyser.NET (MRC-Holland; Amsterdam, The Netherlands). Signal intensities between deleted or duplicated and normal genomic segments from the same subject were compared using Student's $t$ test and considered significant if $p$-value $<0.05$.

\section{Quantitative polymerase chain reaction}

To validate the copy number variations detected by MLPA, quantitative PCR (Q-PCR) was performed. Primers were designed using Primer-BLAST (NCBI) and tested in silico with Beacon Designer Free Edition (Premier Biosoft; Pallo Alto, California, USA), BLAT program (UCSC Genome Browser, Santa Cruz CA, USA) and nucleotide BLAST (NCBI, Bethesda, MD, USA) (Supplementary Table II-5). Prior to Q-PCR, all primers were tested by standard PCR and submitted to agarose gel electrophoresis, according to section II.B. Q-PCR was carried out with the SYBR Green system (Applied Biosystems; Foster City, California, USA) on a 7500 Fast RealTime PCR System apparatus (Applied Biosystems; Foster City, California, USA), as described previously (Ambar and Chiavegatto 2009). Optimal DNA and primer concentrations were determined by titration: for a $24 \mu \mathrm{L}$ reaction, $25 \mathrm{ng}$ DNA and $12.5 \mathrm{pmol}$ of each primer were used with $12 \mu \mathrm{L}$ PCR SYBR Green master mix. A nonTSC control DNA sample was used as a reference. Amplification and melting step (dissociation curve) were performed following standard parameters from the manufacturer program (Supplementary Table II-6). The melting step followed each run to confirm product specificity and the absence of primer dimers (Supplementary Figure II-1).

For copy number calibration, the reaction was normalized to the amplification of an amplicom for the GADPH gene (Glyceraldehyde-3-phosphate dehydrogenase, NM_002046.1, human chr:12). All samples were run in triplicates at least three times, and the data analyzed using the comparative $\Delta \Delta C_{t}$ cycle threshold method (Applied Biosystems; Foster City, California, EUA). Student's $t$ test was applied to compare test and reference samples.

\section{E. Pipeline of in silico and in vivo analysis of DNA variants}

DNA variant nomenclature was according to Human Genome Variation Society (HGVS, Melbourne, Australia) and were verified using both Variant Validator (University of Leicester, Freeman, Hart et al. (2018)) 
and Mutalyzer (LUMC, Wildeman, van Ophuizen et al. (2008)) web tools. Pathogenic DNA variants were defined according to ACMG standards and guidelines. In silico tools that predict damaging effects of missense change over protein structures and those that predict effect on splicing output were considered as a single piece of evidence in sequence interpretation (Richards, Aziz et al. 2015).

To determine the pathogenicity of the DNA variants, the following data banks, web tools and software were consulted: Leiden Open Variation Database (Version 2; Leiden, The Netherlands), Ensembl (release 93 July 2018; Wellcome Trust Sanger Institute; European Bioinformatics Institute, Hinxton, Cambridgeshire, United Kingdom, (Zerbino, Achuthan et al. 2018)), PolyPhen-2 (Adzhubei, Schmidt et al. 2010), Mutation Taster (Schwarz, Rodelsperger et al. 2010), SIFT and PROVEAN (Ng and Henikoff 2006, Kumar, Henikoff et al. 2009, Choi and Chan 2015), PhosphositePlus (Hornbeck, Zhang et al. 2015) and Alamut 2,7-1 (Sophia Genetics, Saint Sulpice, Switzerland).

In silico analysis of DNA variants of uncertain clinical significance (VUS) potentially regulating premRNA splicing was performed using the bioinformatics web tools Acescan2, SpliceAid2 (Piva, Giulietti et al. 2012), Human Splicing Finder 3.1 (HSF -Desmet, Hamroun et al. (2009)) and Alamut 2,7-1 (Sophia Genetics, Saint Sulpice, Switzerland). To identify repetitive elements in the TSC2 genomic sequence the RepeatMasker (Version open-3.0, Institute for Systems Biology, Seattle WA) web tool was used. Information on all identified DNA variants has been deposited at the TSC1 and TSC2 Leiden Open Variation Database (LOVD).

All variants detected by Sanger sequencing or NGS-Panel were submitted to VEP web tool (Figure II-1). First, the transcript reference and mutation code were concatenated (e.g. TSC2 variant c.1832G>A: NM_000548.3) and submitted to standard configuration of VEP, excepted for:

- Transcript database to use: RefSeq transcripts;

- Identifiers: Gene symbol, Transcript version, Protein, UniProt and HGVS;

- Variant and frequency data:

- Frequency data for co-located variants: 1000 Genomes global minor allele frequency, 1000 Genomes continental allele frequencies, ESP allele frequencies and gnomAD (exomes) allele frequencies;

- Filters:

- Restrict results: show one selected consequence per variant.

All variants with frequency above $1 \%$ were filtered out. The remaining variants were verified on LOVD data base for TSC1 and TSC2. If the variant was not present, the impact on protein or splicing was accessed by in silico analysis and functional assessment of mTORC1 pathway activation and/or splicing aberrant edition. 


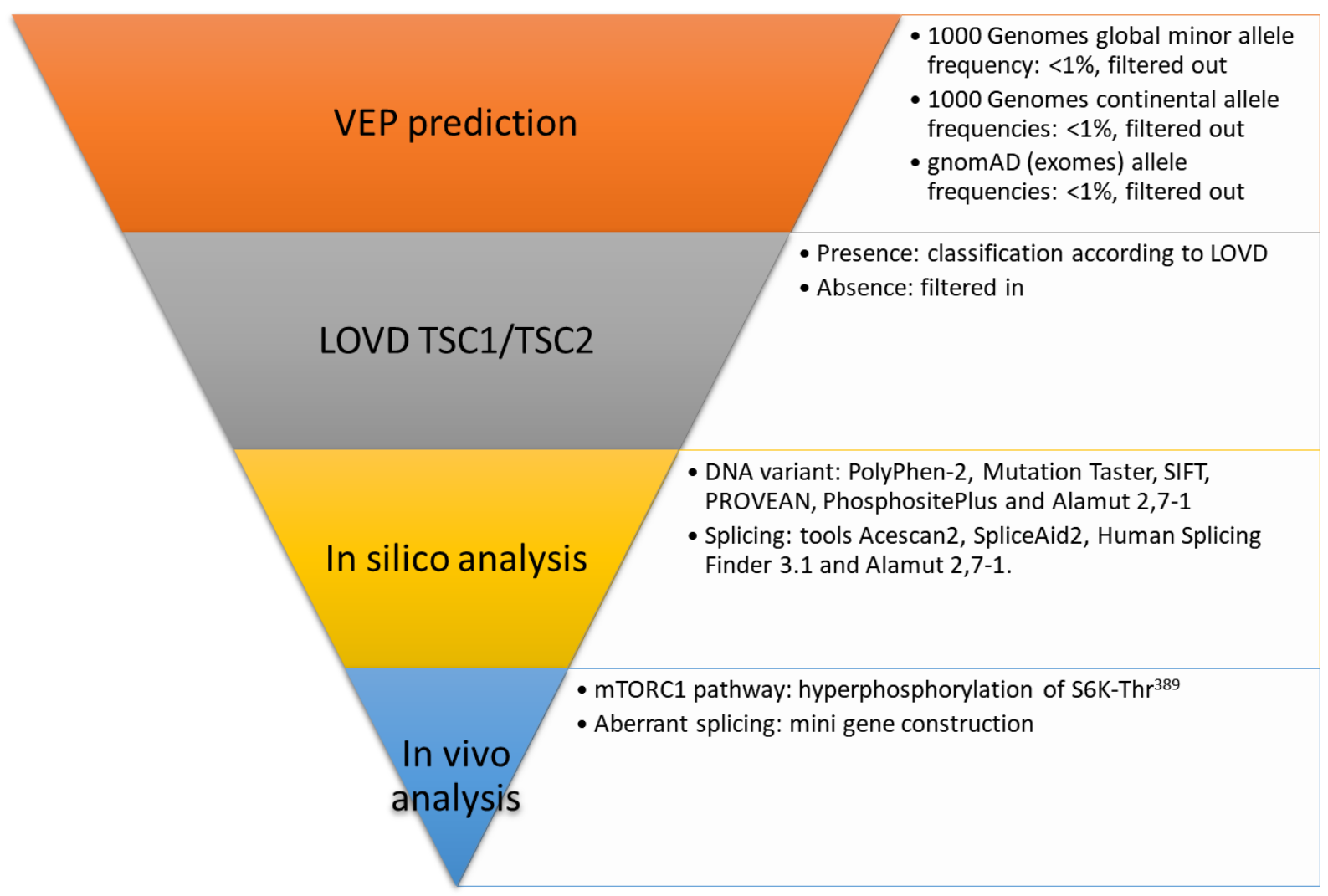

Figure II-1: Workflow of in silico and in vivo analysis of DNA variants detected on TSC1 and TSC2.

Global alignment of Homo sapiens (NP_000539.2), Rattus norvegicus (P49816), and Mus musculus (Q61037) TSC2 C-terminus, and Rap1GAP catalytic domain (Homo sapiens, pdb|1SRQ|A) (Daumke et al., 2004) were performed using UniProtKB BLAST (EMBL-EBI,). The alignments among TSC2 paralogues from the three organisms and Rap1GAP domain were set as reference to identify the position of TSC2 variants. Structural predictions and spatial position of TSC2 variant were generated with PyMOL molecular Graphic System Version 2.1.1 software (Schrödinger, Inc., Cambridge, Massachusetts, USA). Phosphorylation sites of the TSC2 GAP domain was accessed using PhosphoSite Plus $(\mathrm{NIH}$,$) web tool. Supplementary Table II-7 contains a list of$ softwares and websites consulted.

\section{F. Functional assessment of TSC2 VUS missense and in-frame deletion}

TSC2 missense and in-frame deletion functional assessment was performed according to HoogeveenWesterveld, Wentink et al. (2011) and Hoogeveen-Westerveld, Ekong et al. (2012), except for the usage in the present work of the cell line HEK293T that had been genetically modified to have both alleles from TSC1 and TSC2 genes inactivated by CRISPR/Cas9 genome editing, generating the 3H9-1B1 cell line (Dufner-Almeida et 
al., submitted). Briefly, 3H9-1B1 cell line overexpressing full-length TSC2 harboring the assayed DNA variant was tested against the control according to its ability to inhibit mTOR and thus activate S6K phosphorylation.

Full-length TSC2 expression construct encoding the variant of interest was derived by site-directed mutagenesis of the DNA of the human wild-type (WT) TSC2 (NM_000548.3) expression construct previously cloned in the pcDNA3 vector (Hoogeveen-Westerveld, Ekong et al. 2013). Procedures followed the manufacturer protocol (QuikChange II Site-Directed Mutagenesis Kit, Agilent Technologies; Santa Clara, California, USA). Plasmidial DNA that had been submitted to site-directed mutagenesis was isolated and digested with EcoRI, in order to check for the presence of appropriate plasmid DNA. Clone sequence identity was verified by Sanger sequencing, and clones with successful mutagenesis were subcloned, and had the entire open reading frame (ORF) sequenced. Other constructs employed in the functional assay have been described before (Nellist, Sancak et al. 2005), including wild-type (WT) TSC2, TSC2-GFP, TSC1-Myc and WT RPS6KB1 (S6K). Because of the low levels of endogenous expression of $S 6 K$, every transfection included the S6K clone DNA. Transfections were performed with $0.5 \times 10^{6}$ to $1.0 \times 10^{6}$ cells per well of 24 -well dishes, and contained a combination of $0.2 \mu \mathrm{g}$ WT-TSC2, $0.2 \mu \mathrm{g}$ TSC2-GFP, $0.2 \mu \mathrm{g}$ mutant TSC2, $0.4 \mu \mathrm{g}$ TSC1-Myc and 0.2 $\mu \mathrm{g}$ S6K plasmidial DNA, as well as $4.0 \mu \mathrm{g}$ polyethyleneimine (PEI; Sigma-Aldrich, St. Louis, Missouri, USA). Eighteen hours after transfection, the transfection efficiency was verified according to the fluorescence emitted by TSC2-GFP cells on confocal microscopy. Only assays yielding transfection rates higher than $10 \%$ were considered for Western blotting (Figure II-2).

SDM (Site-direct mutagenesis)

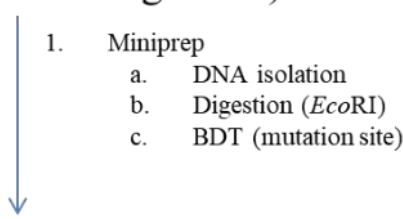

\section{Trasnsfection of $1 \mathrm{~B} 1$ cells}

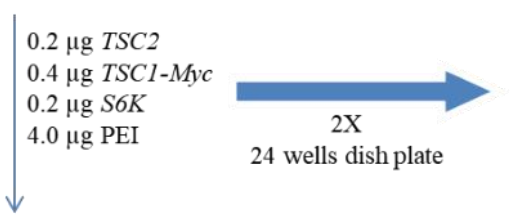

Cell Lysis<smiles>C1CCCCC1</smiles>

SDS-PAGE

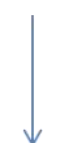

Immunoblot
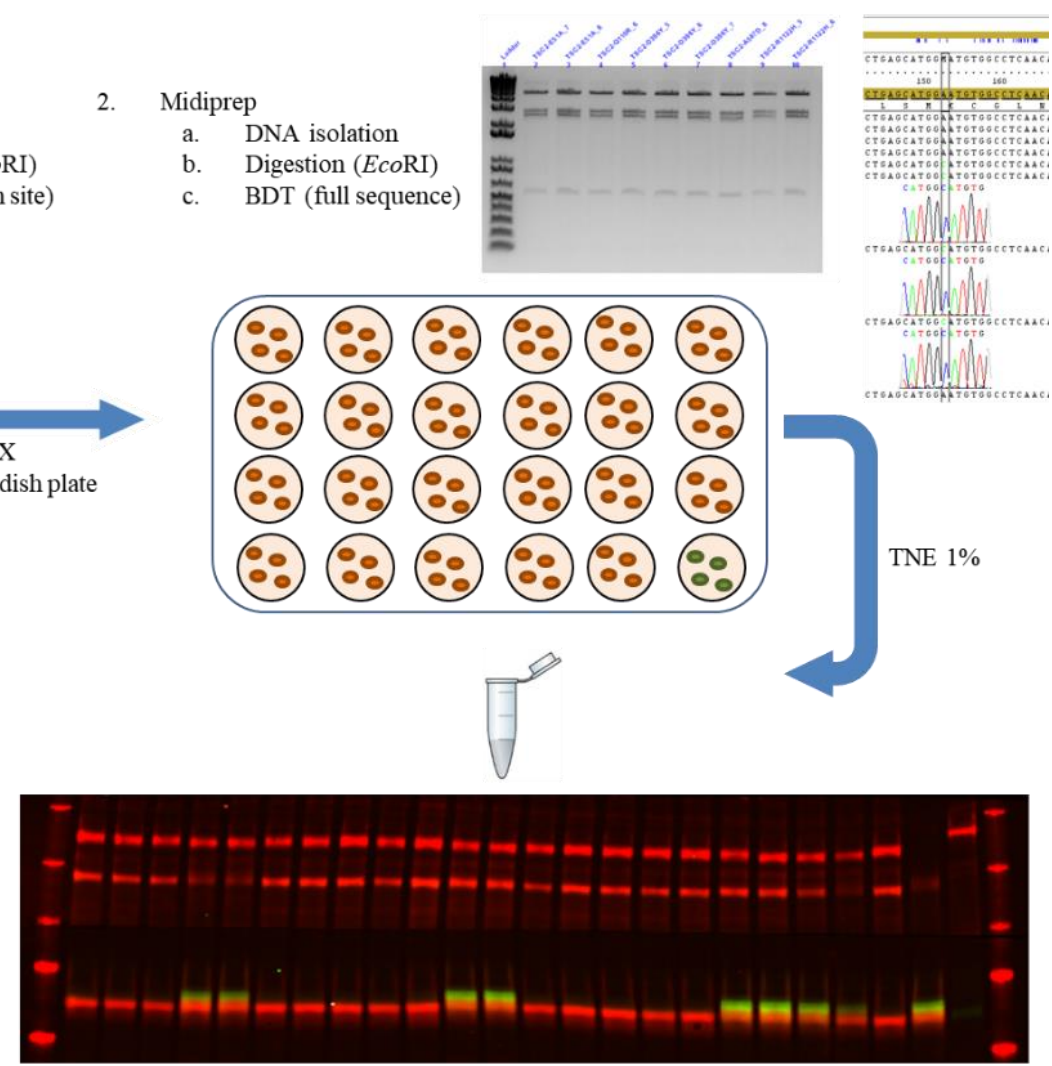
Figure II-2: TSC2 missense and in-frame deletion functional assessment workflow. BDT: Big Dye terminator reaction

For immunoblotting, cells were lysed in $50 \mathrm{mM}$ Tris- $\mathrm{HCl}(\mathrm{pH}$ 7.6), $100 \mathrm{mM} \mathrm{NaCl}, 50 \mathrm{mM} \mathrm{NaF}, 1 \%$ TritonX-100, 1 mM EDTA and complete protease inhibitor cocktail (Roche, Basel, Switzerland) on ice. After 10,000 X $g$ centrifugation for 10 minutes at $4^{\circ} \mathrm{C}$, proteins were submitted to SDS-PAGE in a Criterion ${ }^{\text {TM }}$ TGX ${ }^{\text {TM }}$ Precast Gel (4-15\%) (Bio-Rad, Hercules, California, USA) and transferred to nitrocellulose filters (Bio-Rad, Hercules, California, USA). The membrane was blocked with 5\% powder milk in PBS (Dulbecco's Phosphate Buffer Saline $(0.0095 \mathrm{M})$ - BioWhittaker - Radnor, Pennsylvania, USA) for 30 minutes. Antibodies were from Cell Signaling Technology (1A5 anti-Thr ${ }^{389}$ phospho- p70 S6 kinase (S6K) mouse monoclonal; 9B11 anti-myc tag mouse monoclonal) or have been described previously (anti-TSC1 and -TSC2; van Slegtenhorst, Verhoef et al. (1999)). Secondary antibodies were from Li-Cor Biosciences (Lincoln, Nebraska, USA). Antibody dilutions were in PBS containing 0.1\% Tween 20 (PBST) (Sigma-Aldrich Fine Chemicals, Poole, United Kingdom) according to Table II-2. Primary antibody was incubated for 4 hours or overnight at $8^{\circ} \mathrm{C}$, secondary antibody incubations were for 60 minutes at room temperature. All washes were in PBST with tween. Blots were scanned using either the Odyssey scanner (Li-Cor Biosciences, Lincoln, Nebraska, USA) or the Gel Doc ${ }^{\text {TM }}$ EZ System and Image Lab ${ }^{\text {TM }}$ software (Bio-Rad, Hercules, California, USA). Signal intensities were measured and normalized to the signals corresponding to wild-type TSC2. The experiment was repeated at least three times and Student's $t$ test was used for pairwise comparison of each variant to the WT-TSC2 and the TSC2 p.Arg611GIn variant, a positive control acknowledged to disrupt the TSC1-TSC2 dimer (Nellist, Sancak et al. 2005). Tested DNA variant effect was considered positive if the ratio of $56 \mathrm{~K}-\mathrm{Thr}^{389} /$ total S6K is at least a four-fold compared to WT-TSC2.

Table II-2: Monoclonal and polyclonal antibodies used to functional assessment of TSC2 DNA variants.

\begin{tabular}{lllll}
\hline Color on Blot & Detected Protein & $\begin{array}{l}\text { Mass } \\
(\mathbf{k D a})\end{array}$ & $\begin{array}{l}\text { Primary antibody/species in which it was } \\
\text { generated }\end{array}$ & Dilution fold \\
\hline Red & TSC2-GFP & 225 & Anti-TSC2 $\left(1895^{*}\right) /$ rabbit & $1: 10,000$ \\
\hline Red & TSC2 & 200 & Anti-TSC2 $\left(1895^{*}\right) /$ rabbit & $1: 10,000$ \\
\hline Red & TSC1 & 130 & Anti TSC1 $(2197 * *) /$ rabbit & $1: 5,000$ \\
\hline Green & Myc-Tag & 130 & $9 B 11$ anti-myc tag/mouse & $1: 5,000$ \\
\hline Green & S6K-Thr389 & 70 & 1A5 anti-Thr 389 phospho- S6K/mouse & $1: 2,000$ \\
\hline Red & Total S6K & 70 & Anti-myc tag/rabbit & $1: 2,000$ \\
\hline
\end{tabular}

* Primary antibodies 1/15,000 dilution of 1895 (rabbit polyclonal against TSC2) from (van Slegtenhorst, Nellist et al. 1998)

** Primary antibodies 1/5,000 dilution of 2197 (rabbit polyclonal against TSC1) from (van Slegtenhorst, Nellist et al. 1998)

\section{G. Splicing effect of TSC2 missense and deep intronic variants}

Partial genomic segments containing a DNA variant potentially affecting TSC2 pre-mRNA splicing were cloned in the pcDNA3.1 vector, determining a mini-gene for overexpression in HEK293 cells that had not been genetically modified. The two TSC2 variants tested, c.1361+54_1361+57del in intron 13 and c.4493G>T 
(p.Ser1498lle) in exon 34, defined PCR amplicons containing genomic segments from exon 12 through exon 14, and from exon 33 through exon 35, respectively. Both mutated and wild-type TSC2 alleles were separately cloned in the pcDNA3.1 vector following PCR amplification of DNA from the heterozygous individual for each DNA variant. Forward and reverse primers contained BamHI and Xhol or Not/ restriction sites (Supplementary Table II-8). Sanger sequencing of recombinant plasmidial DNA confirmed cloning of both wild-type alleles, the 4-bp deletion and c.4493G>T variant. HEK293 cells were transfected using lipofectamine 2000 (Thermo Fisher Scientific, Waltham MA, USA). Eighteen hours after transfection, total RNA was isolated with TRIzol, treated with AmpGrade DNase I (1U/ $\mu \mathrm{L}$; Invitrogen, Carlsbad, CA, USA), and reversely transcribed (SuperScript III; Invitrogen, Carlsbad CA, USA). PCR amplification of the CDNA from the mini-gene transcript, with primers specific for the pcDNA3.1 vector (Supplementary Table II-8), yielded products with expected sizes of $210 \mathrm{bp}$ (non-recombinant clone); 596 bp (upon exon 12-14 mini-gene transcript constitutive splicing) or 458 bp (if internal exon is skipped due to the 4-bp deletion); and $896 \mathrm{bp}$ (upon exon 33-35 mini-gene transcript constitutive splicing) or $408 \mathrm{bp}$ (if internal exon is skipped due to the c.4493T mutant allele). Additionally, a nested PCR approach was adopted with primers annealing to TSC2 exons 12 and 14, and 33 and 35 (Supplementary Table II-8). Moreover, primers annealing to exons 8 and 10 were used to amplify TSC2 endogenous transcript (Supplementary Table II-8) as an internal control (327bp). RT-PCR products were verified after electrophoresis in 1.5\% agarose gel, and the images captured by Gel Doc ${ }^{\mathrm{TM}}$ EZ System and Image Lab $^{\text {TM }}$ software (BioRad, Hercules, California, EUA). All bands were isolated from agarose gel and submitted to Sanger sequencing in order to identify the sequence alteration caused by any splicing defect. 


\section{Results}

\section{A. Pathogenic DNA variants}

Our cohort of 117 patients with definite clinical diagnosis of TSC, consisted of 63 male and 54 female individuals. Seventy-five patients were from HC-UFPR, 23 patients from HSCM-SP, and 19 patients from GRAACC-SP. Age at the time of enrolment varied between five months and 19 years (mean: 10.4 years, median: 11 years).

Among the 117 patients, 28 had DNA previously analyzed by Sanger sequencing of the TSC1 gene, having pathogenic alterations detected in seven of them (Dufner-Almeida, 2014). In the present work, the patient cohort and experimental approach have been significantly extended. Overall pathogenic DNA variants were identified in 102 patients (87.2\%), 18 affecting TSC1 (17.6\%), and 84 on TSC2 (82.4\%), and a TSC1:TSC2 mutation ratio of 1:4.7 (Figure II-3A). The number of TSC pathogenic DNA variants is presented in Table II-3 according to the hospital of patient origin.

Table II-3: TSC1 and TSC2 pathogenic DNA variants according to hospital of patient origin.

\begin{tabular}{lllll}
\hline Hospital & HC-UFPR & HSCM-SP & GRAACC-SP & Total \\
\hline Number of patients enrolled & 75 & 23 & 19 & 117 \\
\hline $\begin{array}{l}\text { Patients with pathogenic DNA variants detected } \\
\text { percentage of total) }\end{array}$ & $67(89.33 \%)$ & $22(95.65 \%)$ & $13(68.42 \%)$ & $102(87.17 \%)$ \\
\hline $\begin{array}{l}\text { Patients with } T S C 1 \text { pathogenic DNA variants } \\
\text { percentage of total identified) }\end{array}$ & $7(10.77 \%)$ & $9(40.91 \%)$ & $2(15.38 \%)$ & $18(17.64 \%)$ \\
\hline $\begin{array}{l}\text { Patients with } T S C 2 \text { pathogenic DNA variants } \\
\text { percentage of total identified) }\end{array}$ & $60(89.55 \%)$ & $13(59.09 \%)$ & $11(84.61 \%)$ & $84(82.35 \%)$ \\
\hline TSC1:TSC2 ratio & $1: 8.6$ & $1: 1.4$ & $1: 5.5$ & $1: 4.7$ \\
\hline NMI patients (analyses concluded) & 4 & 1 & 2 & 7 \\
\hline VUS patients (awaiting functional analysis disclosure) & 5 & 0 & 4 & 9 \\
\hline
\end{tabular}

Nonsense (30 patients) and frameshift (29 patients) were the most frequent pathogenic variant categories. Complex mutation and large segmental gene duplication were observed once each in our cohort (Figure II-3B).

Distinct TSC1 alterations comprehended eight frameshift and six nonsense variants, and one large deletion (Figure II-3, and Table II-4). TSC1 exon 15 exhibited four nonsense and three frameshift variants; thus $41.2 \%$ of this gene pathogenic DNA variants (7/17). Two variants, Glu478Lysfs*53 and Arg500*, were identified in two unrelated patients (Table II-4).

Distinct TSC2 pathogenic variants included 20 nonsense, 20 frameshift, 16 splicing and 12 missense DNA variants, six large deletions, four in-frame deletions, one large duplication and one complex mutation. To our knowledge, 52 variants have not been previously reported. Although four of these have been deposited at LOVD TSC2 database by others, no further description is available (Table II-5). We observed a cluster of 
pathogenic variants in TSC2 GAP domain (residues 1562 to 1764) (Figure II-3D), with 20 out of 84 pathogenic variants $(23.8 \%)$ in this segment (Figure II-5).

A

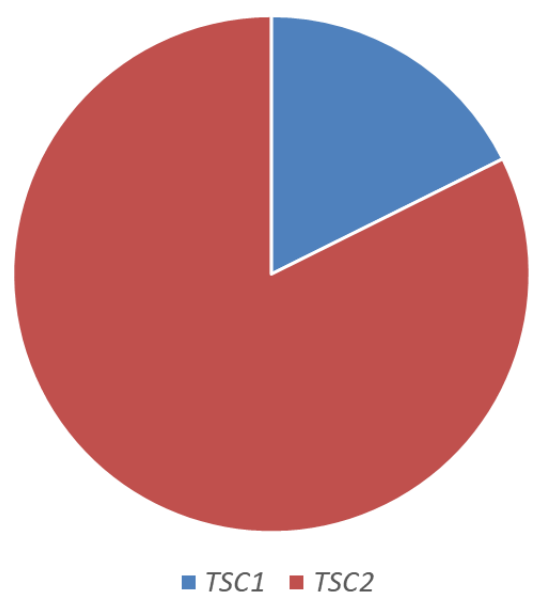

C
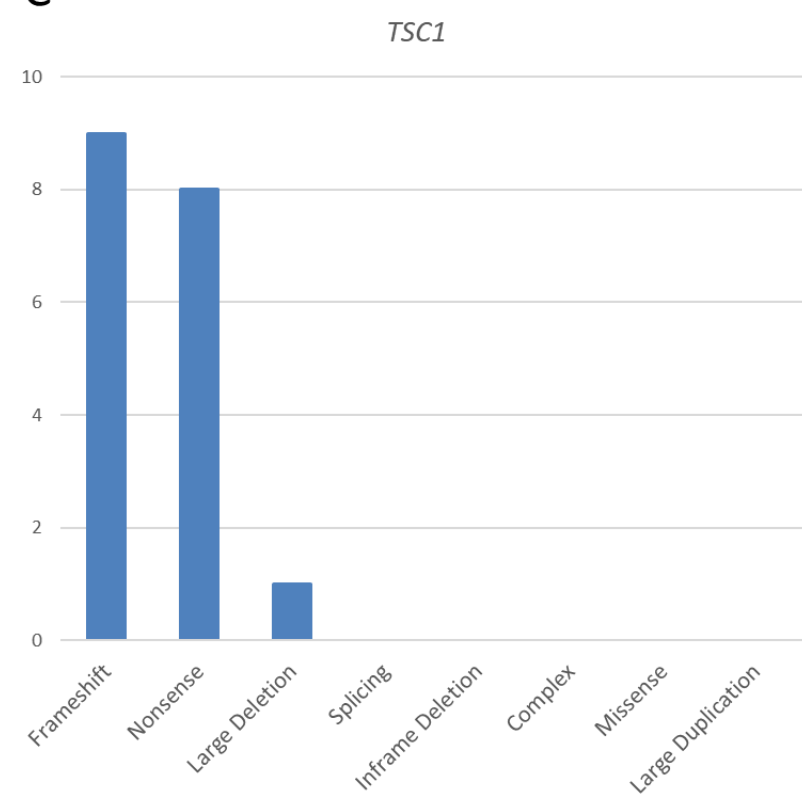

B

Total

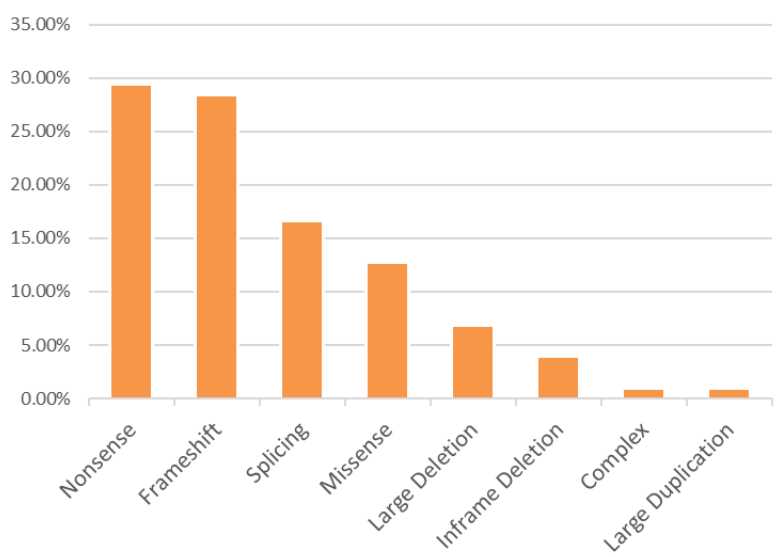

D

TSC2

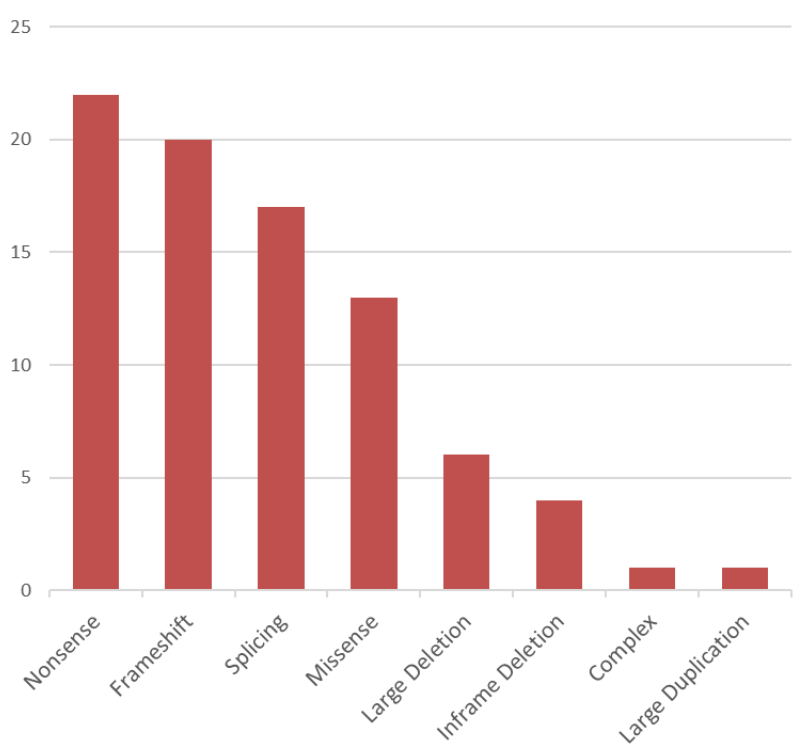

Figure II-3: Overview of the distribution of TSC1 and TSC2 pathogenic DNA variants according to frequency and alteration type. (A) Ratio of TSC1 and TSC2 pathogenic variants. (B) All classes of pathogenic variants found on TSC1 and TSC2. (C) All classes of pathogenic variants found on TSC1. (D) All classes of pathogenic variants found on TSC2 


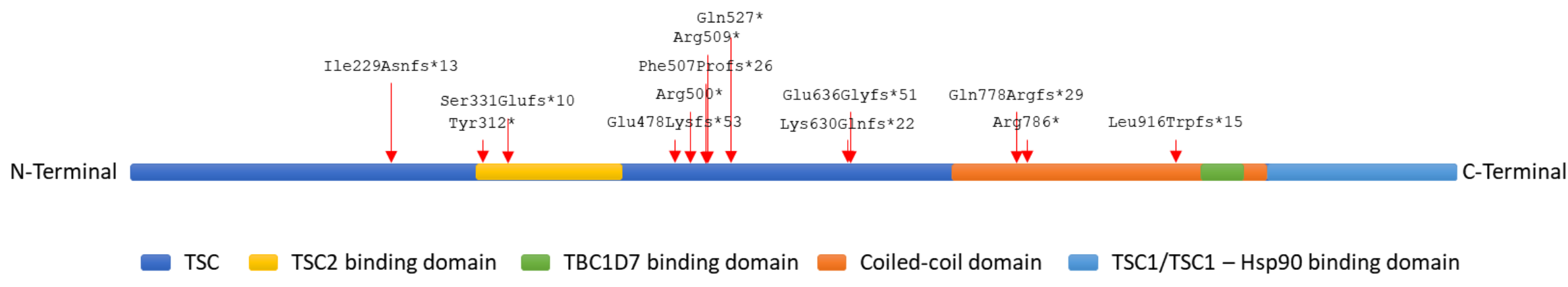

Figure II-4: Schematic representation of TSC1 protein segments and distribution of all TSC1 pathogenic variants according to the amino acid number. 

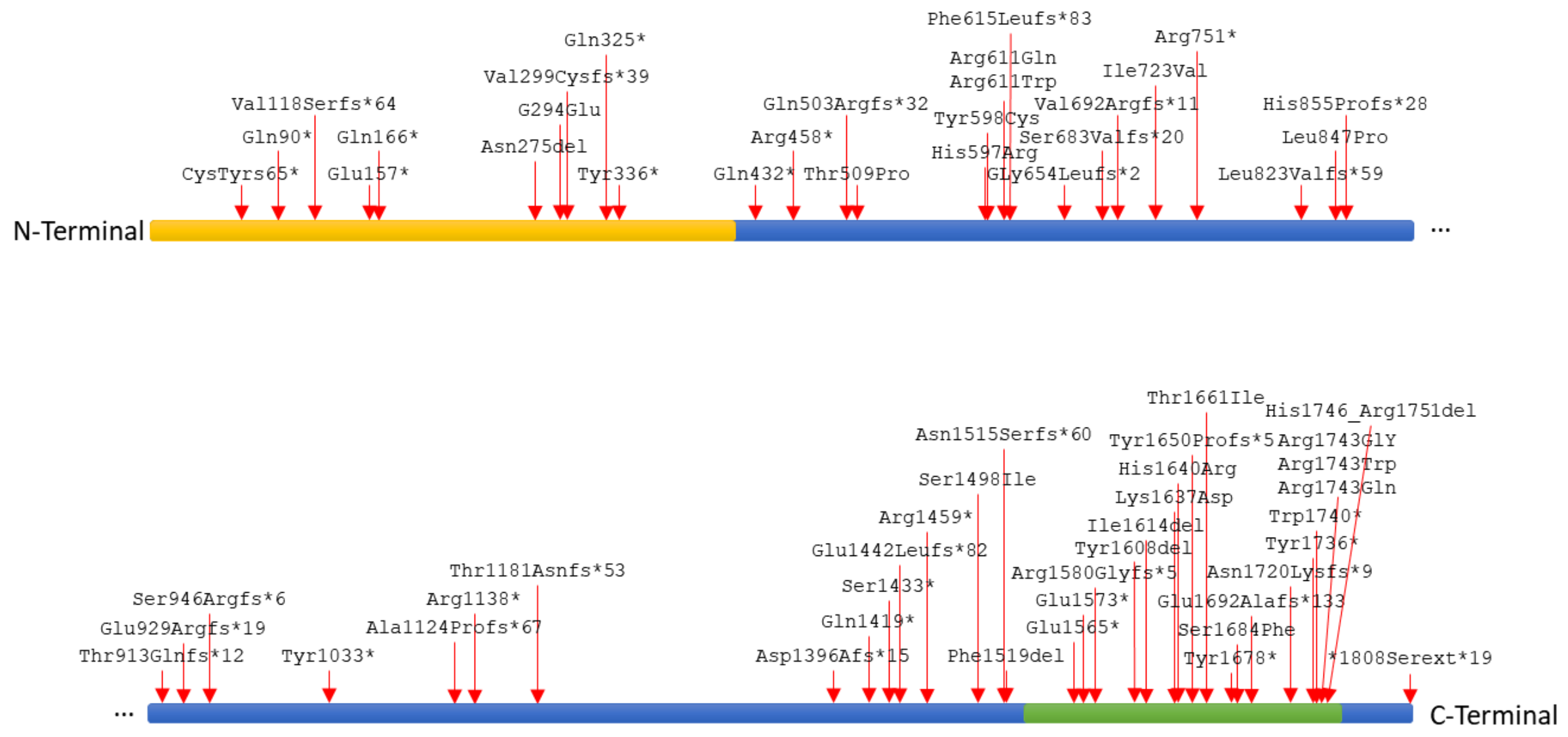

TSC2 $\square$ TSC1 biding domain $\square$ GAP domain

Figure II-5: Schematic representation of TSC2 protein segments and distribution of all TSC2 pathogenic variants according to the amino acid number. Splicing variants are not represented. 
Table II-4: TSC1 pathogenic DNA variants in a cohort of 117 TSC patients.

\begin{tabular}{|c|c|c|c|c|c|}
\hline & DNA variant type & DNA Variant & Location & Patient \# & References $^{f}$ \\
\hline 1. & Frameshift & c.683dupG (p.Ile229Asnfs*13) ${ }^{a}$ & Exon 08 & 36 & - \\
\hline 2. & Frameshift & c.989dupT (p.Ser331Glufs*10) a, g & Exon 10 & 19 & - \\
\hline 3. & Frameshift & c.1431_1434del (p.Glu478Lysfs*53) c, d, g & Exon 14 & 13,78 & $\mathrm{Au}, 2007$ \\
\hline 4. & Frameshift & c.1517_1518dupCC (p.Phe507Profs*26) ${ }^{\text {a, g }}$ & Exon 15 & 17 & - \\
\hline 5. & Frameshift & c.1888_1891del (p.Lys630Glnfs*22) c, d & Exon 15 & 111 & $\begin{array}{l}\text { Rosset, 2017; Tyburczy, 2014; Tsai, 2011; Au, } \\
\text { 2007; Hung, } 2006\end{array}$ \\
\hline 6. & Frameshift & c.1907_1908del (p.Glu636Glyfs*51) & Exon 15 & 122 & $\begin{array}{l}\text { Iyer, 2013; Muzykewicz, 2009; Dabora, 1998; } \\
\text { Kwiatkowska, 1998; vanSlegtenhorst, } 1997\end{array}$ \\
\hline 7. & Frameshift & c.2332del (p.Gln778Argfs $* 29)$ a, c, d & Exon 18 & 79 & - \\
\hline 8. & Frameshift & c.2746delC (p.Leu916Trpfs*15) ${ }^{\text {a, } g}$ & Exon 21 & 10 & - \\
\hline 9. & Nonsense & c. $936 \mathrm{C}>\mathrm{G}\left(\mathrm{p} . \mathrm{Tyr} 312^{*}\right)^{\mathrm{a}, \mathrm{c}, \mathrm{d}}$ & Exon 10 & 93 & - \\
\hline 10. & Nonsense & c. $1525 \mathrm{C}>\mathrm{T}\left(\mathrm{p} . \operatorname{Arg} 509^{*}\right)^{\mathrm{g}}$ & Exon 15 & 5,56 & $\begin{array}{l}\text { Kwiatkowska, } 1998 \text { and 2015; Suspitsin, 2018; } \\
\text { Ismail, 2017; Yang, 2016; Lee, } 2014\end{array}$ \\
\hline 11. & Nonsense & c. $1498 \mathrm{C}>\mathrm{T}\left(\mathrm{p} . \operatorname{Arg} 500^{*}\right)^{\mathrm{g}}$ & Exon 15 & 8,35 & $\begin{array}{l}\text { Tan, 2017; Tyburczy, 2015; Jansen, 2008; Au, } \\
\text { 2007; Sancak, } 2005\end{array}$ \\
\hline 12. & Nonsense & c. $1579 \mathrm{C}>\mathrm{T}\left(\mathrm{p} . \mathrm{Gln} 527^{*}\right)^{\mathrm{c}, \mathrm{d}}$ & Exon 15 & 106 & Voss, 2014; Iyer, 2013 \\
\hline 13. & Nonsense & c. $2356 \mathrm{C}>\mathrm{T}\left(\mathrm{p} . \operatorname{Arg} 786^{*}\right)^{\mathrm{g}}$ & Exon 18 & 16 & $\begin{array}{l}\text { Suspitsin, 2018; Lee, 2014; Au, 2007; Hung, } \\
\text { 2006; Sancak, } 2005\end{array}$ \\
\hline 14. & Nonsense & c. $2626 \mathrm{G}>\mathrm{T}$ (p.Glu876*) & Exon 21 & 82 & - \\
\hline 15. & Large Deletion & c. $\left(737+1 \_738-1\right) \_\left(* 1+\_?\right) \mathrm{del}^{\text {a, b, d }}$ & Exon 9-23 & 29 & - \\
\hline \multicolumn{6}{|c|}{ Variants of unclear significance (VUS) in NMI patients } \\
\hline 16. & Large deletion & c.-234-?_-144+?del & Exon1-intron1 & 25 & - \\
\hline 17. & Splicing & c.738-197T >A & Intron 8 & 60 & \\
\hline 18. & Stop loss & c. $* 1 \mathrm{G}>\mathrm{A}$ & Exon 23 & 100 & - \\
\hline
\end{tabular}

${ }^{\text {a }}$ First description of this variant.

${ }^{\mathrm{b}}$ Next-generation sequencing and MLPA performed for both genes, besides Sanger sequencing.

${ }^{c}$ MLPA performed for both genes, besides Sanger sequencing

${ }^{\mathrm{d}}$ Deletion confirmed by Q-PCR.

${ }^{\mathrm{e}}$ At LOVD database without further information of submission.

${ }^{\mathrm{f}}$ Five most recent references.

${ }^{g}$ Previously described (Dufner-Almeida 2014). 
Table II-5: TSC2 pathogenic DNA variants in a cohort of 117 TSC patients.

\begin{tabular}{|c|c|c|c|c|c|}
\hline & $\begin{array}{l}\text { DNA variant } \\
\text { type }\end{array}$ & DNA Variant & Location & Patient \# & References ${ }^{\text {h }}$ \\
\hline 1. & Nonsense & c. $195 \mathrm{~T}>\mathrm{A}\left(\mathrm{p}\right.$. Cys65*) ${ }^{\mathrm{a}}$ & Exon 03 & 4 & - \\
\hline 2. & Nonsense & c. $268 \mathrm{C}>\mathrm{T}(\mathrm{p} . \mathrm{Gln} 90 *)$ & Exon 04 & 105,120 & $\begin{array}{l}\text { Kwiatkowski, 2015; } \\
\text { Jansen, 2008; Au, 2007; } \\
\text { Yuan, 2007; Hung, 2006 }\end{array}$ \\
\hline 3. & Nonsense & c.469G>T(p.Glu157*) & Exon 05 & 42 & \\
\hline 4. & Nonsense & c. $496 \mathrm{C}>\mathrm{T}\left(\mathrm{p} . \mathrm{G} \ln 166^{*}\right)^{\mathrm{c}}$ & Exon 06 & 94,99 & Niida, 1999 \\
\hline 5. & Nonsense & c. $973 \mathrm{C}>\mathrm{T}\left(\mathrm{p} . \mathrm{Gln} 325^{*}\right)$ & Exon 10 & 52 & Jansen, 2008 \\
\hline 6. & Nonsense & c. $1008 \mathrm{~T}>\mathrm{G}($ p.Tyr336*) & Exon 11 & 115 & Rosset, 2017 \\
\hline 7. & Nonsense & c. $1195 \mathrm{G}>\mathrm{T}($ p.Glu399*) & Exon 12 & 53 & - \\
\hline 8. & Nonsense & c. $1294 \mathrm{C}>\mathrm{T}(\mathrm{p} . \mathrm{G} \ln 432 *)^{\mathrm{a}}$ & Exon 13 & 28 & - \\
\hline 9. & Nonsense & c. $1372 \mathrm{C}>\mathrm{T}\left(\mathrm{p} . \operatorname{Arg} 458^{*}\right)$ & Exon 14 & 108 & $\begin{array}{l}\text { Cai, 2017; Kwiatkowski, } \\
\text { 2015; Tyburczy, 2015; } \\
\text { Tsai, 2011; Au, } 2007\end{array}$ \\
\hline 10. & Nonsense & c. $2251 \mathrm{C}>\mathrm{T}\left(\mathrm{p} . \operatorname{Arg} 751^{*}\right)$ & Exon 21 & 1 & $\begin{array}{l}\text { Cai, 2017; Yang, 2016; } \\
\text { Kwiatkowski, 2015; } \\
\text { Yang, 2014; Kacerovska, } \\
2012\end{array}$ \\
\hline 11. & Nonsense & c. $3099 \mathrm{C}>\mathrm{G}\left(\mathrm{p} . \mathrm{Tyr} 1033^{*}\right)^{\mathrm{a}}$ & Exon 27 & 9 & - \\
\hline 12. & Nonsense & c. $3412 \mathrm{C}>\mathrm{T}$ (p.Arg1138*) & Exon 30 & 92 & $\begin{array}{l}\text { Cai, 2017; Kwiatkowski, } \\
\text { 2015; Tyburczy, 2014; } \\
\text { Hung, 2006; Rendtorff, } \\
2005\end{array}$ \\
\hline 13. & Nonsense & c. $4298 \mathrm{C}>\mathrm{A}\left(\mathrm{p} . \mathrm{Ser} 1433^{*}\right)^{\mathrm{a}}$ & Exon 34 & 26 & - \\
\hline 14. & Nonsense & c. $4375 \mathrm{C}>\mathrm{T}\left(\mathrm{p} . \operatorname{Arg} 1459^{*}\right)^{\mathrm{a}}$ & Exon 34 & 33 & - \\
\hline 15. & Nonsense & c. $4255 \mathrm{C}>\mathrm{T}\left(\mathrm{p} . \mathrm{Gln} 1419^{*}\right)$ & Exon 34 & 67 & $\begin{array}{l}\text { Cai, 2017; Au, 2007; } \\
\text { Roberts, 2004; Langkau, } \\
\text { 2002; Jones, 1999 }\end{array}$ \\
\hline 16. & Nonsense & c. $4693 \mathrm{G}>\mathrm{T}\left(\mathrm{p} . \mathrm{Glu} 1565^{*}\right)^{\mathrm{a}}$ & Exon 37 & 32 & - \\
\hline 17. & Nonsense & c. $4717 \mathrm{G}>\mathrm{T}\left(\mathrm{p} . \mathrm{Glu} 1573^{*}\right)^{\mathrm{a}}$ & Exon 37 & 101 & - \\
\hline 18. & Nonsense & c. $5034 \mathrm{C}>\mathrm{A}\left(\mathrm{p} . \mathrm{Tyr} 1678^{*}\right)^{\mathrm{a}}$ & Exon 39 & 18 & - \\
\hline 19. & Nonsense & c. $5208 \mathrm{C}>\mathrm{G}\left(\mathrm{p} . \mathrm{Tyr} 1736^{*}\right)^{\mathrm{a}}$ & Exon 41 & 48 & - \\
\hline 20. & Nonsense & c.5220G>A (p.Trp1740*) & Exon 41 & 90 & $\begin{array}{l}\text { Kwiatkowski, 2015; } \\
\text { Roberts, } 2002\end{array}$ \\
\hline 21. & Frameshift & c.352del (p.Val118Serfs*64) a, c & Exon 05 & 83 & - \\
\hline 22. & Frameshift & c.894dup (p.Val299Cysfs*39) & Exon 10 & 123 & Giannikou, 2016 \\
\hline 23. & Frameshift & c. 1507 del (p.Gln503Argfs $* 32)$ a, c & Exon 15 & 91 & - \\
\hline 24. & Frameshift & c.1842del (p.Phe615Leufs*83) a & Exon 18 & 22 & - \\
\hline 25. & Frameshift & c.2073dup (p.Val692Argfs*11) a & Exon 19 & 41 & - \\
\hline 26. & Frameshift & c.2046dup (p.Ser683Valfs $* 20)^{a}$ & Exon 19 & 71 & - \\
\hline
\end{tabular}




\begin{tabular}{|c|c|c|c|c|c|}
\hline 27. & Frameshift & c.1959_1960del (p.Gly654Leufs*2) & Exon 19 & 103 & Li, 2011 \\
\hline 28. & Frameshift & c.2467_2476delinsGTGGATGA (p.Leu823Valfs*59) a, b & Exon 21 & 20 & - \\
\hline 29. & Frameshift & c.2563dup (p.His855Profs $* 28)^{\text {a }}$ & Exon 23 & 73 & - \\
\hline 30. & Frameshift & c.2737_2739delinsC (p.Thr913Glnfs*12)a & Exon 24 & 3 & - \\
\hline 31. & Frameshift & c.2784del (p.Glu929Argfs*19) ${ }^{\text {a }}$ & Exon 25 & 39 & - \\
\hline 32. & Frameshift & c.3370del (p.Ala1124Profs*67) ${ }^{a}$ & Exon 29 & 110 & - \\
\hline 33. & Frameshift & c.3541dup (p.Thr1181Asnfs $* 53)^{a}$ & Exon 30 & 86 & - \\
\hline 34. & Frameshift & c.4324_4327delinsCTTCT (p.Glu1442Leufs*82) a & Exon 34 & 57 & - \\
\hline 35. & Frameshift & c.4187del (p.Asp1396Alafs*15) a, c & Exon 34 & 95 & - \\
\hline 36. & Frameshift & c.4544_4547del (p.Asn1515Serfs*60) & Exon 35 & 69 & $\begin{array}{l}\text { Kwiatkowski, 2015; Li, } \\
\text { 2011; Au, 2007; Hung, } \\
\text { 2006; Ali, 2005 }\end{array}$ \\
\hline 37. & Frameshift & c. 4738 del (p.Arg1580Glyfs $* 5)^{\text {a }}$ & Exon 37 & 112 & - \\
\hline 38. & Frameshift & c.4947_4948insCCATTGT (p.Tyr1650Profs*5) ${ }^{a}$ & Exon 38 & 31 & - \\
\hline 39. & Frameshift & c.5159dup (p.Asn1720Lysfs*9) ${ }^{\text {a }}$ & Exon 39 & 11 & - \\
\hline 40. & Frameshift & c.5075_5078del (p.Glu1692Alafs*133) & Exon 40 & 49 & Roberts, 2004 \\
\hline 41. & Missense & c. $1525 \mathrm{~A}>\mathrm{C}$ (p.Thr509Pro) $\mathrm{g}, \mathrm{j}$ & Exon 15 & 118 & - \\
\hline 42. & Missense & c.1790A>G (p.His597Arg) & Exon 17 & 68 & $\begin{array}{l}\text { Wang, 2016; Hardy, } \\
\text { 2012; vanEeghen, 2012; } \\
\text { Dunlop, 2011; }\end{array}$ \\
\hline 43. & Missense & c.1793A>G (p.Tyr598Cys) & Exon 17 & 109 & $\begin{array}{l}\text { Hoogeveen-Westerveld, } \\
2013\end{array}$ \\
\hline 44. & Missense & c. $1832 \mathrm{G}>\mathrm{A}(\mathrm{p} . \operatorname{Arg} 611 \mathrm{Gln})^{\mathrm{c}}$ & Exon 17 & 38 & $\begin{array}{l}\text { Suspitsin, 2018; Cai, } \\
\text { 2017; Ishikawa, 2017; } \\
\text { Kwiatkowski, 2015; Liu, } \\
2015\end{array}$ \\
\hline 45. & Missense & c. $1831 \mathrm{C}>\mathrm{T}(\mathrm{p} . \operatorname{Arg} 611 \mathrm{Trp})$ & Exon 17 & 58 & $\begin{array}{l}\text { Kwiatkowski, 2015; } \\
\text { Tyburczy, 2015; You, } \\
\text { 2013; vanEeghen, 2012; } \\
\text { Hoogeveen-Westerveld, } \\
2011\end{array}$ \\
\hline 46. & Missense & c.2540T $>C(p . L e u 847 \text { Pro })^{j}$ & Exon 22 & 46 & $\begin{array}{l}\text { Suspitsin, 2018; } \\
\text { Hoogeveen-Westerveld, } \\
\text { 2012; Li, } 2011\end{array}$ \\
\hline 47. & Missense & c.4909_4911delinsGAC (p.Lys1637Asp) ${ }^{a}$ & Exon 38 & 85 & - \\
\hline 48. & Missense & c.4919A>G (p.His1640Arg) ${ }^{a}$ & Exon 38 & 72 & - \\
\hline 49. & Missense & c.5024C>T (p.Pro1675Leu) & Exon 39 & 64,65 & $\begin{array}{l}\text { van Eeghen, 2012; } \\
\text { Hoogeveen-Westerveld, } \\
\text { 2013; Sancak, } 2005\end{array}$ \\
\hline 50. & Missense & c.5227C >G (p.Arg1743Gly) a, c & Exon 41 & 43 & $\begin{array}{l}\text { Zhang, 2013; } \\
\text { Kwiatkowski,2015 }\end{array}$ \\
\hline 51. & Missense & c.5227C>T (p.Arg1743Trp) & Exon 41 & 77 & $\begin{array}{l}\text { Papadopoulou, 2018; } \\
\text { Suspitsin, 2018; Cai, }\end{array}$ \\
\hline
\end{tabular}




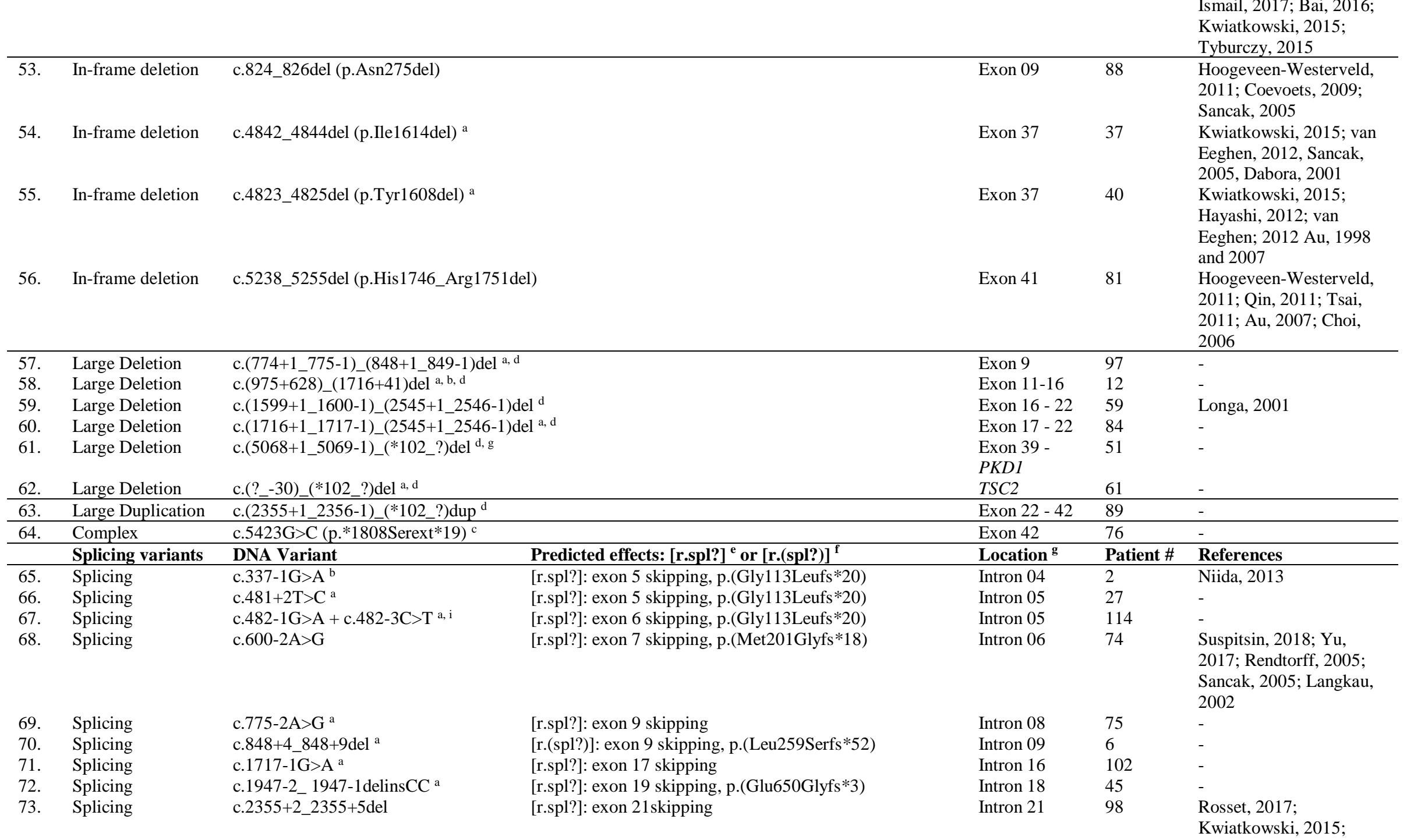




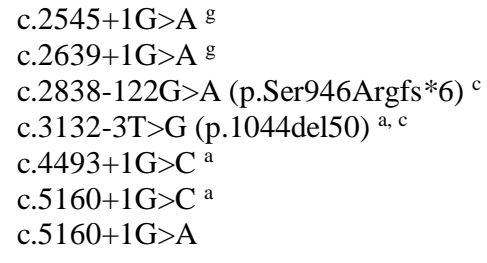

[r.(2837_2838ins2838-120_2838-1)], p.(Ser946Argf *6) Intron 25

[r.(spl?)]: in-frame exon 28 skipping, p.(1044del50)

Intron 27

Intron 34

[r.spl?]: exon 40 skipping, p.(Asp1690Aspfs*6)

Intron 40

[r.spl?]: exon 40 skipping, p.(Asp1690Aspfs*6)

Intron 40

LeCaignec, 2009;

Sancak, 2005; Jones,

1999

\begin{tabular}{|c|c|c|c|c|c|}
\hline \multicolumn{6}{|c|}{ Variants of unclear significance (VUS) in NMI patients } \\
\hline 81. & Synonym & $\mathrm{c} .1119 \mathrm{G}>\mathrm{A}(\mathrm{p} .=)$ & Exon 11 & 104 & - \\
\hline 82. & Missense & c.1443G>T (p.Glu481Asp) & Exon14 & 66,68 & - \\
\hline 83. & Missense & c. $1663 \mathrm{G}>\mathrm{C}(\text { p.Ala555Pro })^{\mathrm{j}}$ & Exon 16 & 50 & - \\
\hline 84. & Missense & c. $4493 \mathrm{G}>\mathrm{T}$ (p.Ser1498Ile) & Exon 34 & 30 & \\
\hline 85. & Missense & c.5051C>T (p.Ser1684Phe) & Exon 39 & 116 & \\
\hline 86. & Missense & c.5228G $>C$ (p.Arg1743Pro) & Exon 41 & 116 & \\
\hline 87. & Splicing & c. $4005+153 \mathrm{~T}>\mathrm{A}$ & Intron 33 & 63 & - \\
\hline 88. & Complex & c. $* 44 * 45$ insGTCA & Exon 42 & 100 & - \\
\hline
\end{tabular}

${ }^{a}$ First description of this variant.

${ }^{\mathrm{b}}$ Next-generation sequencing and MLPA performed for both genes, besides Sanger sequencing.

${ }^{c}$ MLPA performed for both genes, besides Sanger sequencing.

Deletion or duplication confirmed by Q-PCR.

e [r.spl?]: RNA was not analysed but the change is expected to affect splicing.

${ }^{\mathrm{f}}[\mathrm{r} .(\mathrm{spl}$ ?)]: RNA was not analysed but the change might affect splicing.

${ }^{g}$ At LOVD database without further information of submission.

${ }^{\text {h }}$ Five most recent references.

${ }^{\mathrm{I}}$ Found in trans

Under functional analysis 


\section{B. $\quad$ TSC2 missense and in-frame deletion variants}

Initially, 20 distinct TSC2 putative missense variants and five in-frame deletions had general population frequency lower than 0.1 , and were predicted to be disease-causing variants by Mutation Taster, PolyPhen, PROVEAN, SIFT and Human Splicing Finder bioinformatics web tools. Among the missense variants, nine have been previously reported by other groups: His597Arg, Tyr598Cys, Arg611Trp, Arg611GIn, Leu847Pro, Pro1675Leu, Arg1743Gly, Arg1743Trp and Arg1743GIn (Table II-5). Arg611Trp, Arg611GIn, Arg1743Trp and Arg1743GIn have been reported in family cases (Wilson, Ramesh et al. 1996, Beauchamp, Banwell et al. 1998, Niida, Lawrence-Smith et al. 1999, Nellist, Sancak et al. 2005, Au, Williams et al. 2007, Hoogeveen-Westerveld, Wentink et al. 2011). Pathogenicity had been confirmed by functional assays for six cases: His597Arg, Arg611Trp, Arg611Gln, Lys1637Asp (M. Nellist, Erasmus Medical Centre, The Netherlands, personal communication), Arg1743Trp and Arg1743GIn (Nellist, Sancak et al. 2005, Hoogeveen-Westerveld, Wentink et al. 2011). Moreover, TSC2 Tyr598Cys has been reported as likely pathogenic because its functional assay had yielded a S6K-Thr ${ }^{389} / \mathrm{S6K}$ ratio of intermediate value. It was significantly higher than that of wild-type TSC2, but significantly lower than that of pathogenic TSC2 Arg611GIn (Hoogeveen-Westerveld, Ekong et al. 2013). DNA variants Leu847Pro and Arg1743Gly had been reported as pathogenic without further information (Li, Zhou et al. 2011, Hoogeveen-Westerveld, Ekong et al. 2012, Kwiatkowski, Palmer et al. 2015). Finally, variants Thr1661lle and Ser1684Phe had yielded non-pathogenic evidence in the mTOR-based functional assay (M. Nellist, Erasmus Medical Centre, The Netherlands, personal communication). In summary, ten out of 19 missense DNA variants identified in our cohort with significantly low or null frequency in population databases had been functionally assessed, yielding six pathogenic, two non-pathogenic results, and one borderline evidence (Figure II-6). 


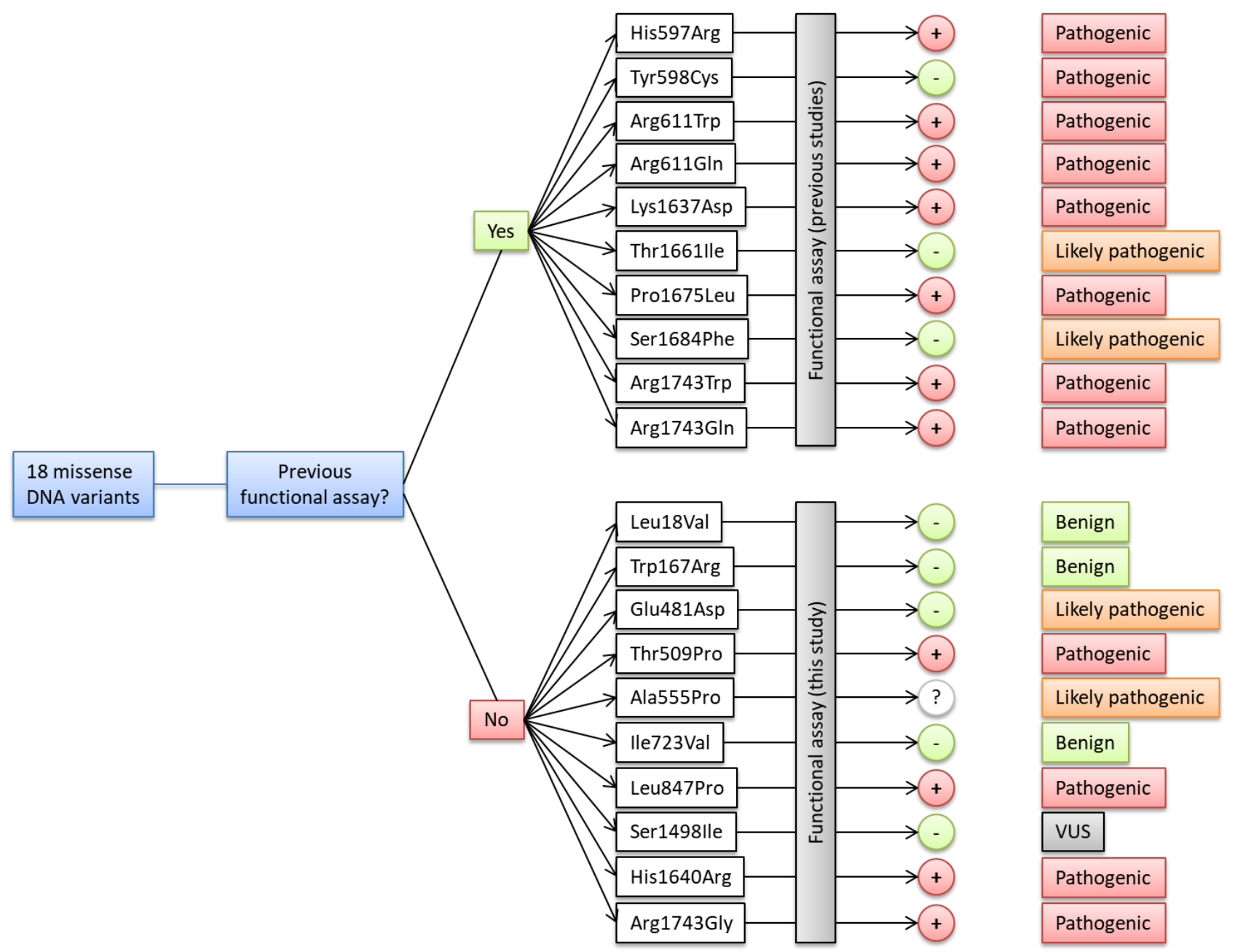

Figure II-6: Workflow of the pathogenicity classification of 20 TSC2 missense DNA variants. VUS: variant of uncertain significance. ?: Variant under functional investigation. Classification according to ACMG standards before the functional assay of the present study

We adopted the American College of Medical Genetics and Genomics (ACMG, Bethesda, MD, USA) criteria for pathogenicity assessment of missense DNA variants and criteria combination (Richards, Aziz et al. 2015; Table II-6, Supplementary Table II-9 and 11).(Richards, Aziz et al. 2015) Besides the six variants that had kept mTORC1 activated in the functional assay, two others (Tyr598Cys and Arg1743Gly) fulfilled the criteria for pathogenicity (Supplementary Table II-9). Moreover, the two variants that inactivated mTOR in the functional assay (Thr1661lle and Ser1684Phe) classified as likely pathogenic. Among the nine cases of missense DNA variants for which no functional data were available, four were likely benign (Leu18Val, Trp167Arg, Glu481Asp and Ala555Pro). Two of them (Trp167Arg and Ala555Pro) were from patients with TSC1 or TSC2 nonsense variants, one detected in this study and the other from a case available at LOVD. The third likely benign variant Leu18Val was from the same patient with the missense variant Ser1498lle. Four additional missense DNA variants classified as likely pathogenic (Thr509Pro, Ile723Val, Leu847Pro and His1640Arg), and 
Ser1498Ile was considered a variant of uncertain significance, according to ACMG criteria (Table II-6 and Figure II-6).

Five in-frame deletions identified by Sanger sequencing in the TSC2 gene were not detected in any database consulted. All in-frame deletions have been reported before (Table II-5). Asn275del and His1746_Arg1751del had been functionally assessed displaying T389/S6K phosphorylation ratios significantly higher than that of wild-type TSC2 (Sancak, Nellist et al. 2005, Hoogeveen-Westerveld, Wentink et al. 2011). DNA variants c.4842_4844del (p.lle1614del) and c.4823_4825del (p.Tyr1608del) have been described as pathogenic (Au, Rodriguez et al. 1998, Dabora, Jozwiak et al. 2001, Sancak, Nellist et al. 2005, Au, Williams et al. 2007) but no functional assay had been conducted. These two in-frame deletion variants, lle1614del and Tyr1608del, were predicted to be disease-causing variants by Mutation Taster and PROVEAN bioinformatics web tools. The fifth in-frame deletion, c.4527_4529del (p.Phe1510del) on exon 35, was not present in 1000 Genomes Project neither gnomAD databases, but according to ClinVar it classifies as benign or likely benign. At TSC2LOVD database, this variant has been reported more than 25 times and classified as probable neutral as the functional assessment did not detect $\mathrm{S} 6 \mathrm{~K}-\mathrm{Thr}^{389}$ phosphorylation significantly higher than wild-type TSC2 (Sancak, Nellist et al. 2005, Hoogeveen-Westerveld, Wentink et al. 2011). 
Table II-6: Pathogenicity criteria for classification of TSC2 missense ( $N=20)$ and in-frame deletion ( $N=5$ ) DNA variants with low population frequency (Richards, Aziz et al. 2015

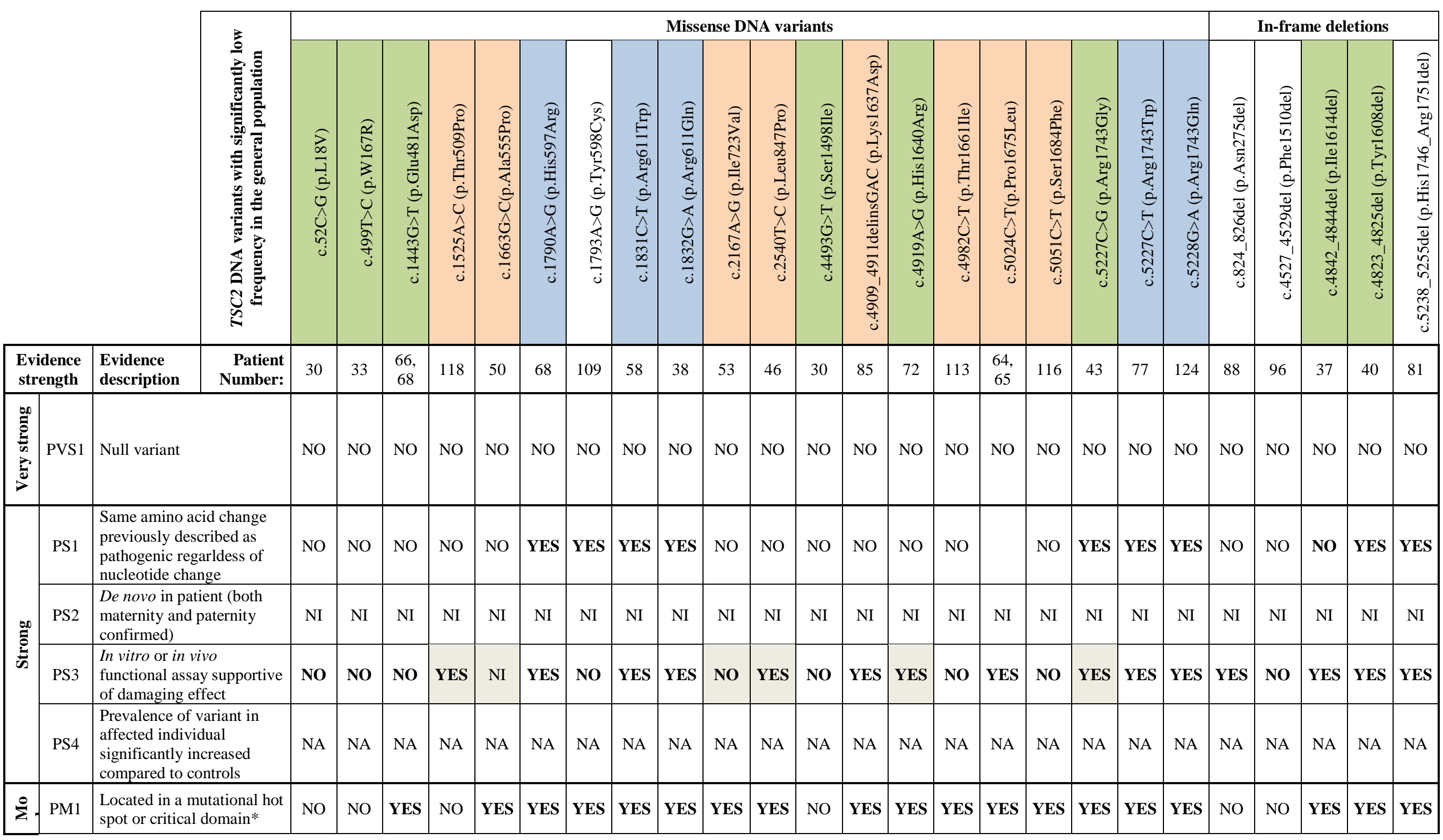




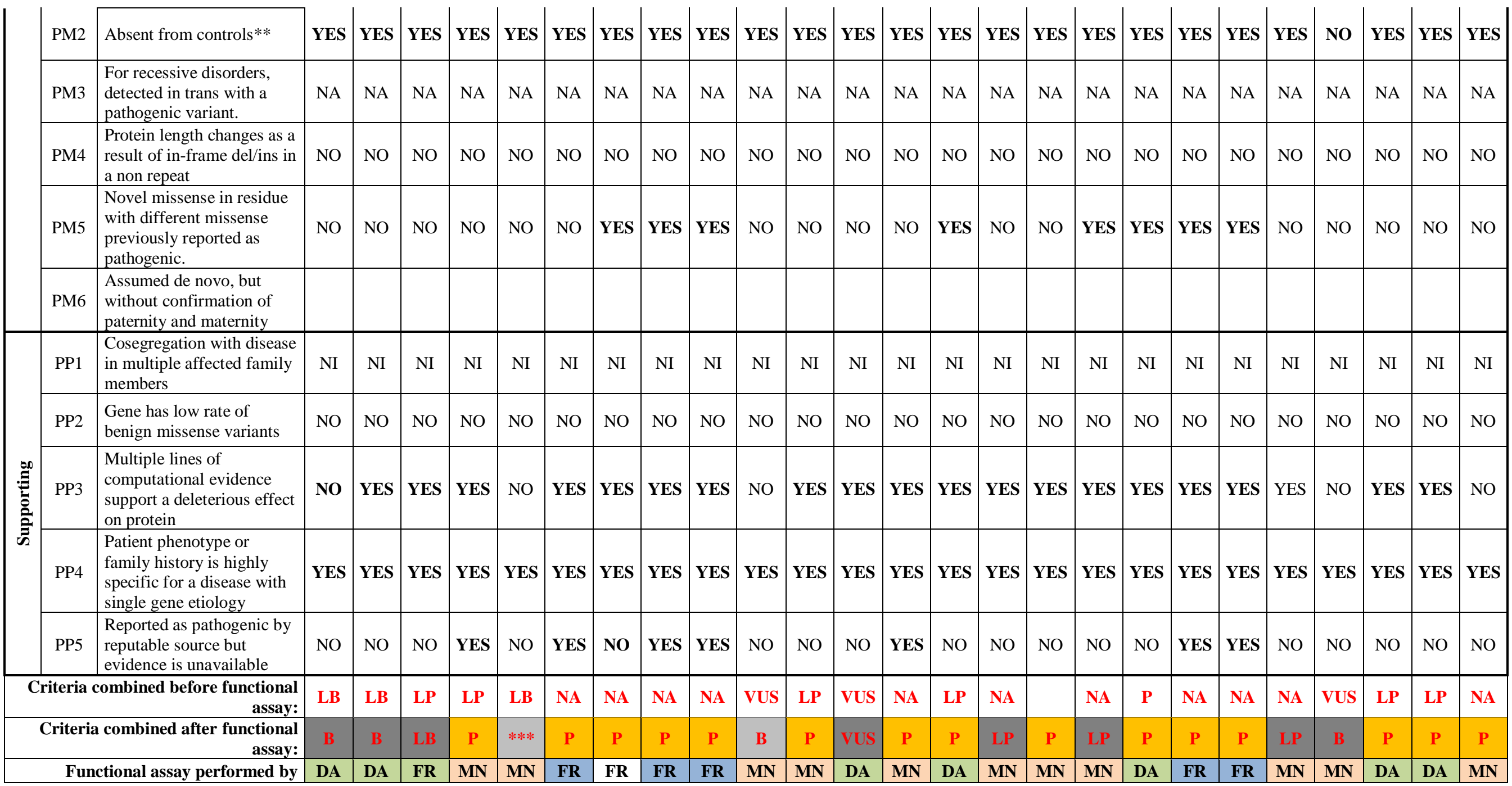

NO: negative criterion; YES: positive criterion; NA: criterion does not apply; NI: criterion not investigated

P: pathogenic; LP: likely pathogenic; B: benign; LB: likely benign; VUS: variant of uncertain significance.

DA: Dufner-Almeida, this study. MN: Mark Nellist, personal communication. FR: former reports as referenced on the text.

*TSC2 domains (approximate amino acid numbers): DUF3384 (55-469); Tuberin domain - hamartin binding (555-903); Rap-GAP domain (1562-1748).

**1000 genomes, Exome Variation and EXAc. 
The mTORC1 activity-based functional assay was employed to evaluate the pathogenicity of five missense variants - c.52C>G (p.Leu18Val), c.499T>C (p.Trp167Arg), c.4493G>T (p.Ser1498lle), c.5227C>G (p.Arg1743Gly) and c.4919A>G (p.His1640Arg), a splicing variant leading to in-frame deletion of exon 27 c.3132-3T>G (p.1044del50) - and two in-frame deletions identified in the present study c.4842_4844del (p.lle1614del) and c.4823_4825del (p.Tyr1608del). TSC2 variants Ser1498Ile, His1640Arg and 1608del had TSC2 levels significantly decreased to WT-TSC2 (Figure II-7B). No variant impaired TSC1 levels as Arg611GIn did (Figure II-7C). For all variants tested, total S6K levels were not different from those of the WT-TSC2 (Figure II-7D). Finally, 1044del50, 1614del, His1640Arg, 1608del and Arg1734Gly had relative S6K-Thr $389 /$ S6K signal ratio similar to the Arg611GIn and were significantly higher than TSC2-WT (Figure II-7E), thereby considered pathogenic variants. On the other hand, the relative S6K-Thr ${ }^{389} / \mathrm{S6K}$ signal for Ser1698lle was significantly reduced comparatively to Arg611GIn ( $p=0.002$; Student's t-test with Bonferroni correction), and nearly fourfold increased comparatively to WT-TSC2 as although without statistic significance $(p=0.042)$, hence remaining a variant of unclear significance (VUS). Three missense variants have been later tested by Dr Nellist group using the same functional assay. Two had pathogenicity confirmed (Thr509Pro and Leu847Pro) and one (lle723Val) was confirmed as benign (data not shown).

In summary, the functional assay confirmed pathogenicity for a putative pathogenic DNA variant (Arg1743Gly), disclosed pathogenicity for three likely pathogenic missense DNA variant (His1640Arg, ), and did not change Ser1498Ile classification as VUS nor three likely benign variants (Leu18Val,Trp167Arg and Ile723Val). Two missense variants remain to be functionally assessed (Glu481Asp and Ala555Pro). Thus, 11 out of 18 functionally tested missense DNA variants in TSC2 had pathogenicity confirmed. Among the twelve pathogenic missense variants ascertained according to the ACMG pathogenicity criteria (Table II-6), ten were confirmed by the functional assay and one had a borderline result (Figure II-6). 


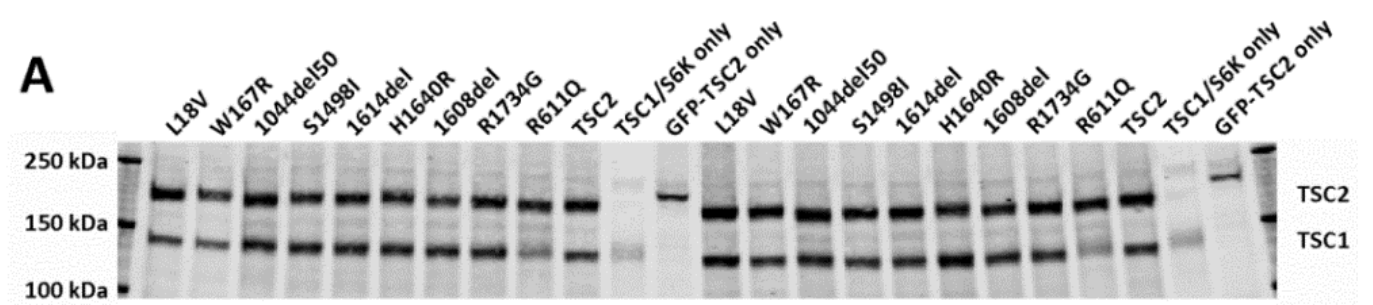

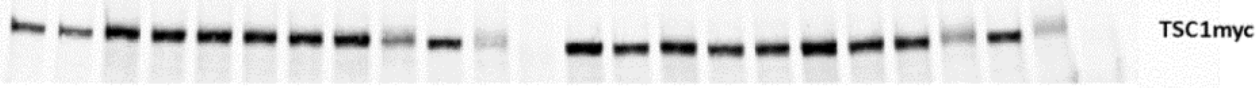

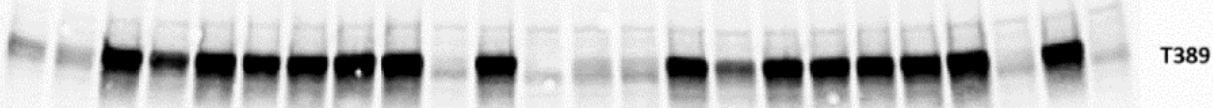
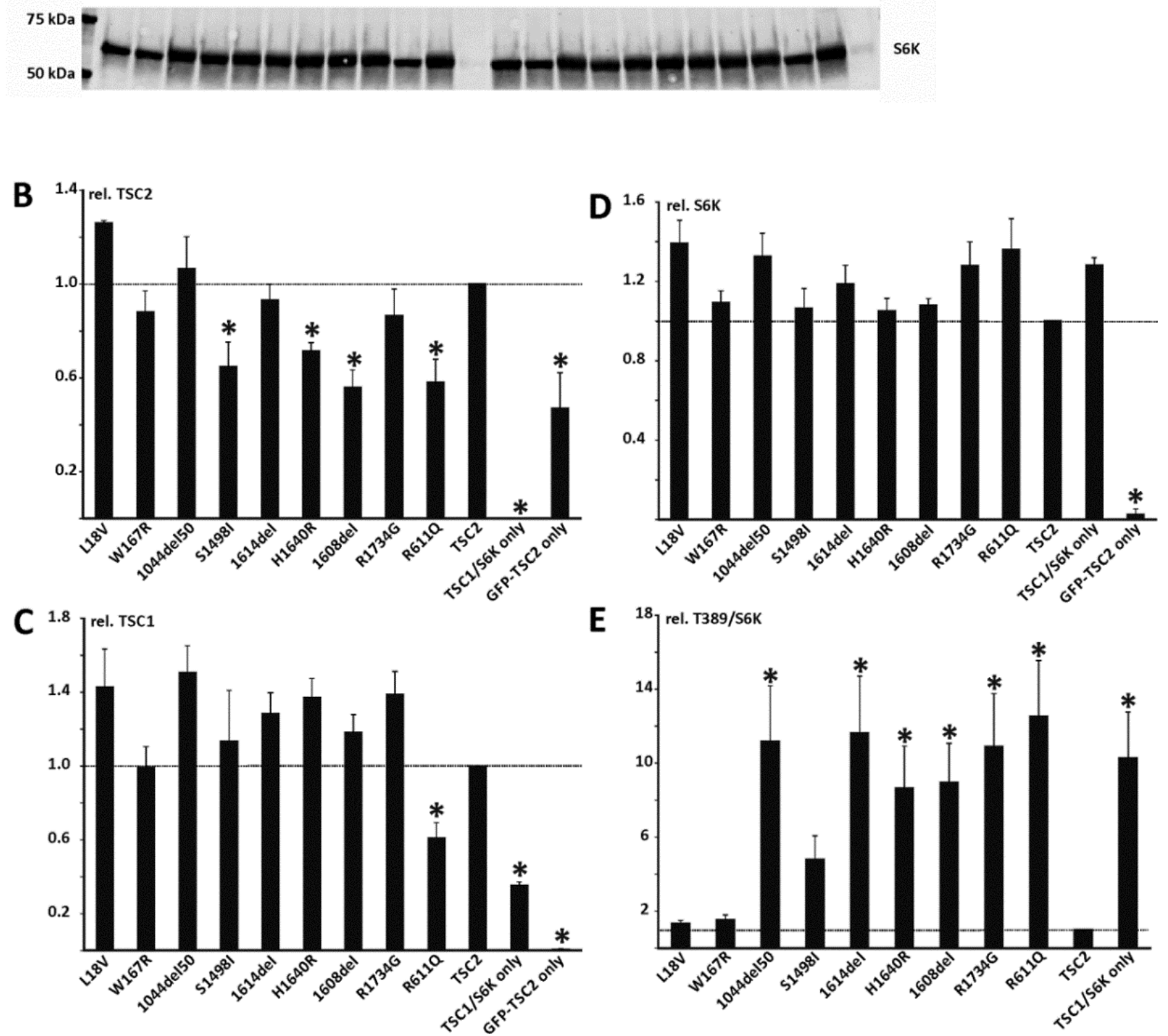

Figure II-7: Functional assessment of the TSC2 (NM_000548.3) variants c.52C>G (p.Leu18Val), c.499T>C (p.Trp167Arg), c.3132_3282del (p.1044_1094del), c.4493G>T (p.Ser1498lle), c.4840_4842del (p.1614del), c.4919A>G (p.His1640Arg), c.4823_4825del (p.1680del) and c.5227C>G (p.Arg1743Gly). 3H9-1B1 (TSC2/TSC1 double knockout HEK 293T) cells were transfected with the indicated TSC2 variant expression constructs, together with expression constructs for myc-tagged TSC1 and S6K. The known pathogenic TSC2 c.1832G>A (p.Arg611Gln) variant (R611Q) and cells not expressing any TSC2 (TSC1/S6K only) were included as controls. To monitor transfection efficiency, cells were transfected with an expression construct for GFP-TSC2 (GFP-TSC2 only). Twenty-four hours after transfection cells were harvested and the cleared cell lysates analyzed by immunoblotting (A). The signals for TSC2, TSC1, total S6K (S6K) and T389- 
phosphorylated S6K (T389) were determined per variant, relative to the wild-type control (TSC2) in two independent, duplicate experiments. The mean TSC2 (B), TSC1 (C) and S6K (D) signals and mean T389/S6K ratio (E) are shown for each variant. In each case the dotted line indicates the signal/ratio for wild-type TSC2 (1.0). Error bars represent the standard error of the mean. TSC2 variants for which the TSC2, TSC1 or S6K signals were significantly reduced compared to wild-type TSC2, or for which the T389/S6K was significantly increased compared to wild-type TSC2, are indicated with an asterisk ( $\mathrm{P}<0.05$; Student's paired t-test). Amino acid changes are given according to TSC2 CDNA reference transcript sequence NM_000548.3.

\section{Variants that affect splicing}

Sixteen TSC2 pathogenic splice variants were identified in the cohort. Fifteen variants mapped to canonical splice sites: eight at splice donor site and seven at splice acceptor site. Cloning and sequencing of c.482-1G>A and c.482-3C >T variants from the same patient disclosed they were in trans. Therefore, c.482$3 \mathrm{C}>\mathrm{T}$ should be considered a rare SNV. One variant mapped to a deep intronic sequence (Figure II-8) and has been detected in two patients (Table II-5).

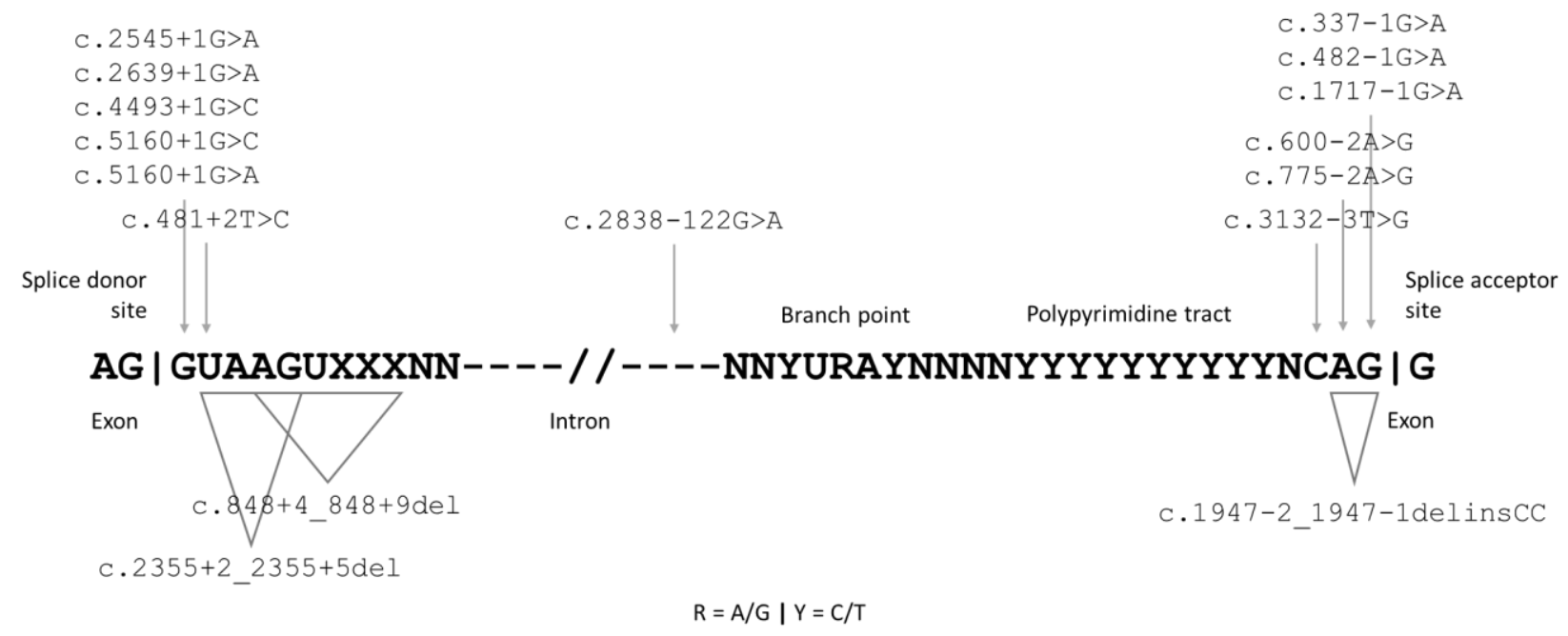

Figure II-8: Schematic representation of distribution of pathogenic TSC2 splice variants. Intron canonical splice sites are indicated (donor and acceptor) as well as conserved branch point and downstream polypyrimidine tract. Vertical bar: border between exon and intron.

The DNA variant c.4493G>T (p.Ser1498Ile) disclosed a non-pathogenic result in the functional mTORbased assay (Figure II-7). On the other hand, splicing-based softwares predicted the c.4493G>T variant could potentially impact on TSC2 canonical splicing site choice leading to skipping of exon 34 . Therefore, we interrogated whether c.4493G $>$ T could affect splicing of TSC2 exon 34. The genomic segment of TSC2 exon 33 through the end of exon 34 from the heterozygous patient DNA was cloned in PCDNA3 vector, and each clone, wild-type or harbouring the nucleotide substitution, was used to transfect the human cell line HEK293. Twenty-four hours after transfection, RT-PCR was performed with vector primers. A 210-bp product was observed for the non-recombinant vector cDNA. If the interrogated exon 34 had been skipped upon splicing of the mini-gene primary transcript, the expected RT-PCR products would have $408 \mathrm{bp}$, whereas constitutive splicing products would be expected with $896 \mathrm{bp}$. 
We did not detect products with the length corresponding to the skipped exon. The constitutive splicing product was present for the wild-type allele. Moreover, an additional RT-PCR reaction was included as an endogenous control, limiting a 327 bp product from TSC2 exon 8 to 10 mRNA. The endogenous internal control was equally amplified in all samples, including the non-recombinant vector sample (Figure II-9). Slowmigrating bands were observed for all samples. All RT-PCR bands were isolated from agarose gel and Sanger sequencing confirmed them as the canonical splicing product of exon 34 mini-gene. We concluded that, in HEK293 cells, the TSC2 variant c.4493T allele did not affect the splicing of the mini-gene. This variant found in patient 14 was observed in patient 50, classified as NMI. As there the frequency of the in the population and two homozygous individuals have already been observed according to the gnomAD database.

A

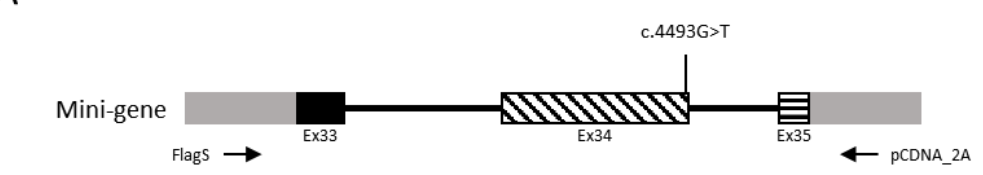

Non-recombinant | $210 \mathrm{bp}$

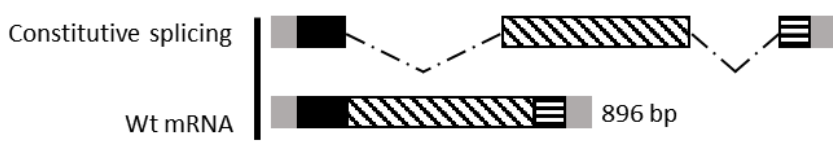

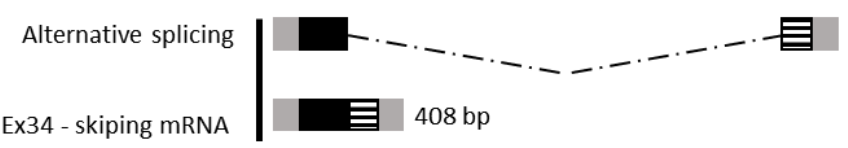

B

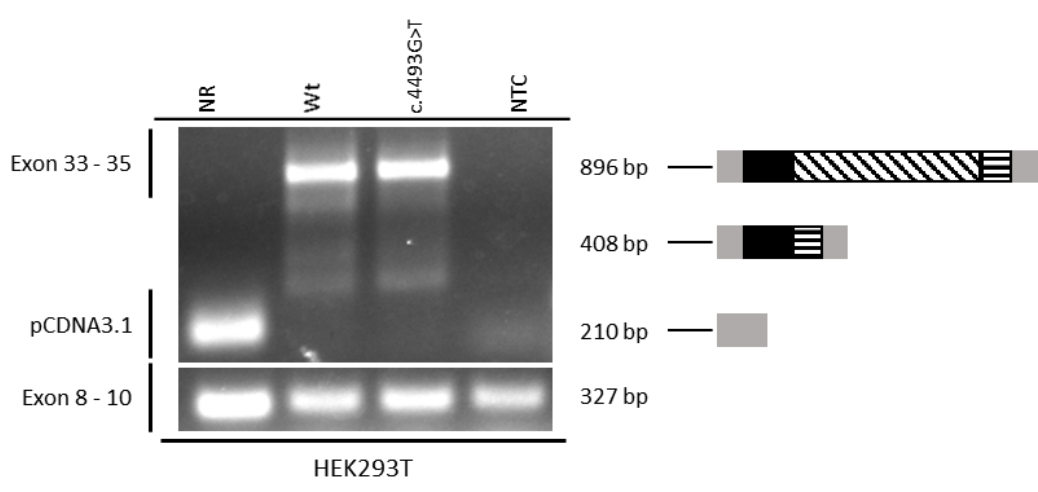

Figure II-9: Functional evaluation of a substitution DNA variant in the TSC2 gene (c.4493G>T p.Ser1498Ile variant). (A) Scheme of predicted constitutive and alternative splicing skipping exon 34 of the mini-gene. The top segment refers to a scheme of the mini-gene: grey blocks are part of vector sequence flanking the mini-gene insert, black blocks are external exons, striped block is the interrogated exon, and black lines are introns. Primer location and identification are indicated by arrows. The location of patient DNA va riant that originated the mutant clones, c.4493G $>T$, is indicated. The expected RT-PCR products of cDNA from cells transfected with either wildtype or deletion clones are indicated, as well as for the non-recombinant clone. (B) Electrophoresis in 1.5\% agarose gel of RT-PCR products from HEK293 cell line transfected with non-recombinant vector (NR), wild-type mini-gene (WT), deletion clones and NTC (notemplate control). 'Exon $33-35$ ' indicates the gel region expected to contain the bands corresponding to the RT-PCR from constitutive and alternative splicing of the mini-gene. Exon 8-10 indicate the RT-PCR internal control. 
As mentioned, one deep intronic DNA variant (c.2838-122G >A) was considered pathogenic as it had been functionally assessed. It altered splicing of the minigene primary transcript, consequently shifting the reading frame (p.Ser946Argfs*6) (Nellist, Brouwer et al. 2015). In an NMI patient, a variant of uncertain significance (c.1361+54_1361+57del) was identified in TSC2 intron 13. This DNA variant has not, to our knowledge, been observed in the general population. In spite of the lack of prediction by the Human Splicing Finder tool, both computational tools Acescan2/Rescue and Spliceaid disclosed the 4-bp sequence c.1361+54_1361+57 embedded within a putative intronic splicing enhancer. Of note, Spliceaid recognized it as a potential binding site for the trans-acting protein factor CUG triplet repeat RNA-binding protein 1 / ELAVlike family member 1(CUGBP1/CELF1). The genomic segment of TSC2 exon 12 through the end of exon 14 from the heterozygous patient DNA was cloned in pCDNA3 vector, and each clone, wild-type or harbouring the deletion, was used to transfect the human cell lines HEK293. If the exon 12 was skipped upon splicing of the mini-gene primary transcript, the expected RT-PCR products would be $430 \mathrm{bp}$, whereas constitutive splicing products would be expected to be $534 \mathrm{bp}$. We observed no products with the length corresponding to the skipped exon. The constitutive splicing product was present for the wild-type. The endogenous control from TSC2 exons 8 to 10 mRNA was equally amplified in all samples (Figure II-10). All RT-PCR bands were isolated form agarose gel and Sanger sequencing confirmed it as the constitutive splicing product of exon 12-14 minigene. We conclude that in HEK293 cells, the TSC2 variant c.1361+54_1361+57del allele did not change the splicing output of the mini-gene. Therefore, that variant was considered benign. 
A

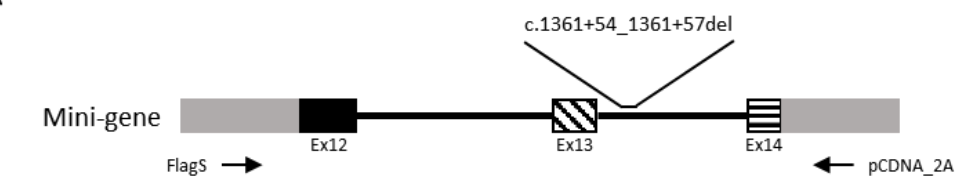

Non-recombinant | $210 \mathrm{bp}$

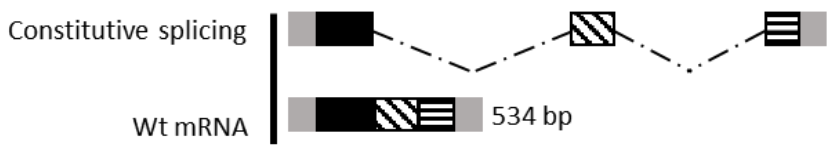

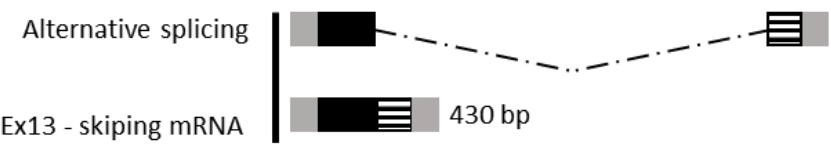

B

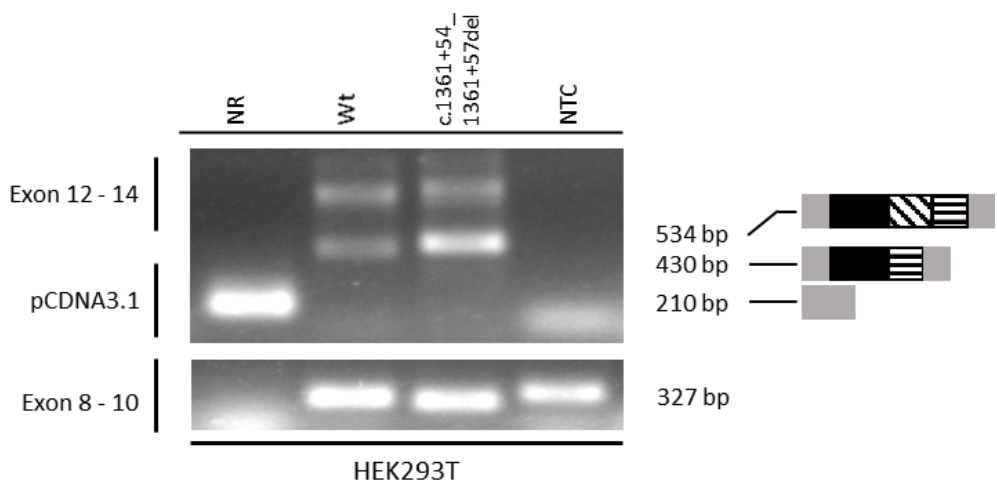

Figure II-10: Functional evaluation of a deep intronic DNA variation in the TSC2 gene (c.1361+54_1361+57del variant). (A) Scheme of predicted constitutive and alternative splicing, involving skipping of exon 12, of the c.1361+54_1361+57del variant mini-gene. In The top segment refers to a scheme of the mini-gene is shown: grey blocks are part of vector sequence flanking the mini-gene insert, black blocks are external exons, the striped block is the interrogated exon to be tested, and black lines are introns. Primers location and identification are indicated by arrows. The location of the patient DNA variant that originated the mutant clones, c.1361+54_1361+57del, is indicated. The expected RT-PCR products of cDNA from cells transfected with either wild-type or deletion clones are indicated, as well as for the non-recombinant clone. (B) Electrophoresis in 1.5\% agarose gel of RT-PCR products from HEK293 cell line transfected with non-recombinant vector (NR), wild-type mini-gene (WT), deletion clones and NTC (no-template control). 'Exon $12-14$ ' indicates the gel region expected to contain the bands corresponding to the RT-PCR from constitutive and alternative splicing of the mini-gene. Exon $8-10$ indicate the RT-PCR internal control.

Data recently available at gnomAD (Massachusetts Institute of Technology, MIT, Cambridge, MA, USA) revealed the frequency of the c.1361+54_1361+57del allele as $0.2 \%$, as it has been detected in 467 out of 207,454 individuals.

\section{Large segmental gene deletions and duplications}

After Sanger sequencing, DNA from 43 patients with missense variants, in-frame deletions or no mutation identified (NMI) was submitted to MLPA of both TSC1 and TSC2 genes. Nine copy number variations 
(CNV) were identified in NMI patients: two in the TSC1 and seven in the TSC2 genes. All CNV were validated by quantitative PCR (Q-PCR). MLPA and corresponding Q-PCR results are presented in Table II-7 and Figure II-11. Both methods inferred somatic mosaicism for the deletions in patients \#25 (TSC1 c.-234-?_-144+?del), \# 12 (TSC2 c.(975+628)_(1716+41)del), and \#51 (TSC2 c.(5068+1_5069-1)_(*102_?)del) (Table II-7).

Table II-7: MLPA results and Q-PCR validation of TSC1 and TSC2 CNV.

\begin{tabular}{|c|c|c|c|c|c|}
\hline \multirow[b]{2}{*}{ Patient } & \multicolumn{2}{|c|}{ MLPA } & \multirow[b]{2}{*}{ ps; p } & \multicolumn{2}{|r|}{ Q-PCR } \\
\hline & CNV & Position & & Position & rq (sem); p \\
\hline & \multicolumn{5}{|c|}{$T S C 1$} \\
\hline 25 & c.-234-?_-144+?del & Exon 1 & $0.80 ;<0.01$ & Exon 1 & $0.79(0.05) ;<0.01$ \\
\hline \multirow[t]{2}{*}{29} & c. $\left(737+1 \_738-1\right) \_\left(* 1+\_?\right)$ del a & Exon $9-23$ & $0.60 ;<0.01$ & Exon 17 & $0.40(0.01) ;<0.01$ \\
\hline & \multicolumn{5}{|c|}{$\mathrm{TSC} 2$} \\
\hline 97 & c.(774+1_775-1)_(848+1_849-1)del a & Exon 9 & $0.58 ;<0.01$ & Exon 9 & $0.45(0.03) ;<0.01$ \\
\hline 12 & c. $(975+628) \_(1716+41) \mathrm{del}$ a & Exon $11-16$ & $0.74 ;<0.01$ & Exon 12 & $0.62(0.07) ; 0.03$ \\
\hline 59 & c.(1599+1_1600-1)_(2545+1_2546-1)del & Exon $16-22$ & $0.56 ;<0.01$ & Exon 19 & $0.54(0.01) ;<0.01$ \\
\hline 84 & c. $\left(1716+1 \_1717-1\right) \_\left(2545+1 \_2546-1\right)$ del & Exon $17-22$ & $0.56 ;<0.01$ & Exon 19 & $0.63(0.02) ;<0.01$ \\
\hline 89 & c. $\left(2355+1 \_2356-1\right) \_\left(* 102 \_?\right)$ dup & Exon $22-42$ & $1.47 ;<0.01$ & Exon 41 & $2.43(0.07) ;<0.01$ \\
\hline 51 & c. $\left(5068+1 \_5069-1\right) \_\left(* 102 \_?\right)$ del & Exon 39 - PKD1 & $0.68 ;<0.01$ & Exon 41 & $0.75(0.02) ;<0.01$ \\
\hline 61 & c. $\left(? \_-30\right) \_\left(* 102 \_?\right)$ del & $\mathrm{TSC} 2$ & $0.54 ;<0.01$ & Exon 12 & $0.50(0.03) ;<0.01$ \\
\hline
\end{tabular}

MLPA position refers to the extension of the detected deletion or duplication, while Q-PCR position refers to exon tested to validate MLPA.

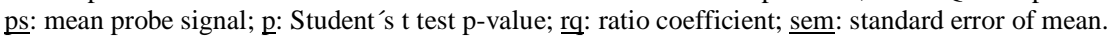

${ }^{\text {a }}$ First description of this variant

NextGENe analysis of TSC1 and TSC2 NGS data from three patients with segmental deletions confirmed the large TSC1 deletion encompassing exons 9 through 23 (patient \#29), and the TSC2 deletion between exons 11 and 16 (patient \#12). The mosaic segmental deletion containing TSC1 exon 1 (patient \#25) was not identified as this exon was not covered by the Nextera probe set. In addition, no other DNA variant was identified by NGS in these patients.

Due to the somatic mosaicism of the TSC1 exon 1 deletion, the absence of coding region in this exon, and previous reports interrogating its clinical significance (van den Ouweland, Elfferich et al. 2011), it was not classified as pathogenic, and patient \#25 remained with NMI. The other eight CNV classified as pathogenic. 
A
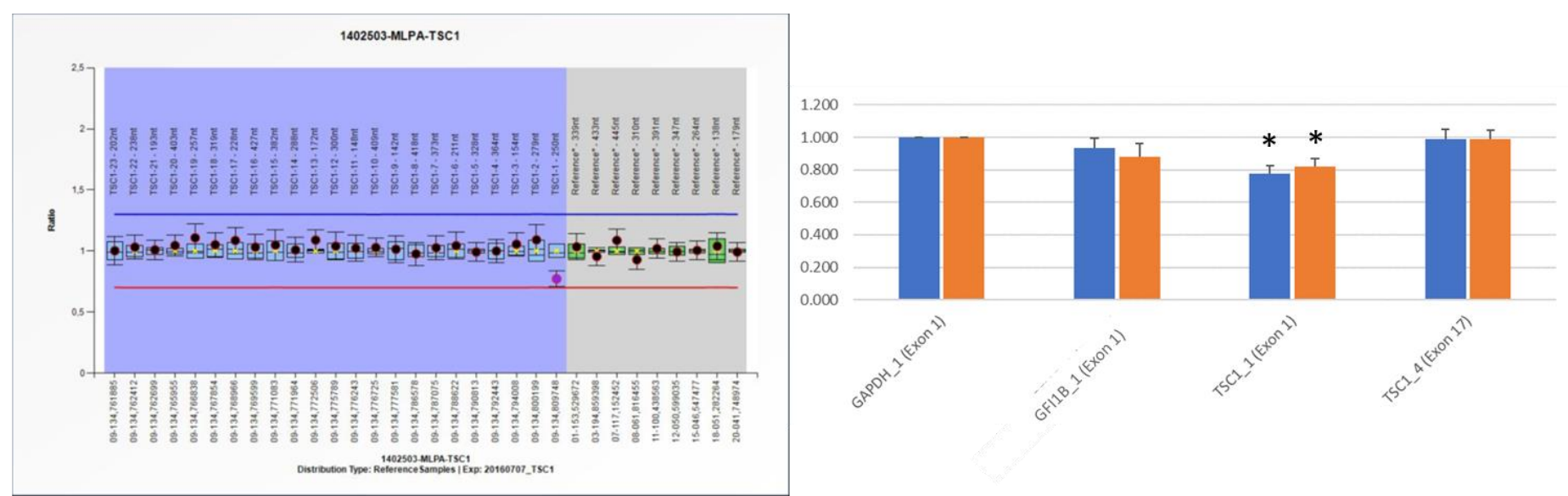

B
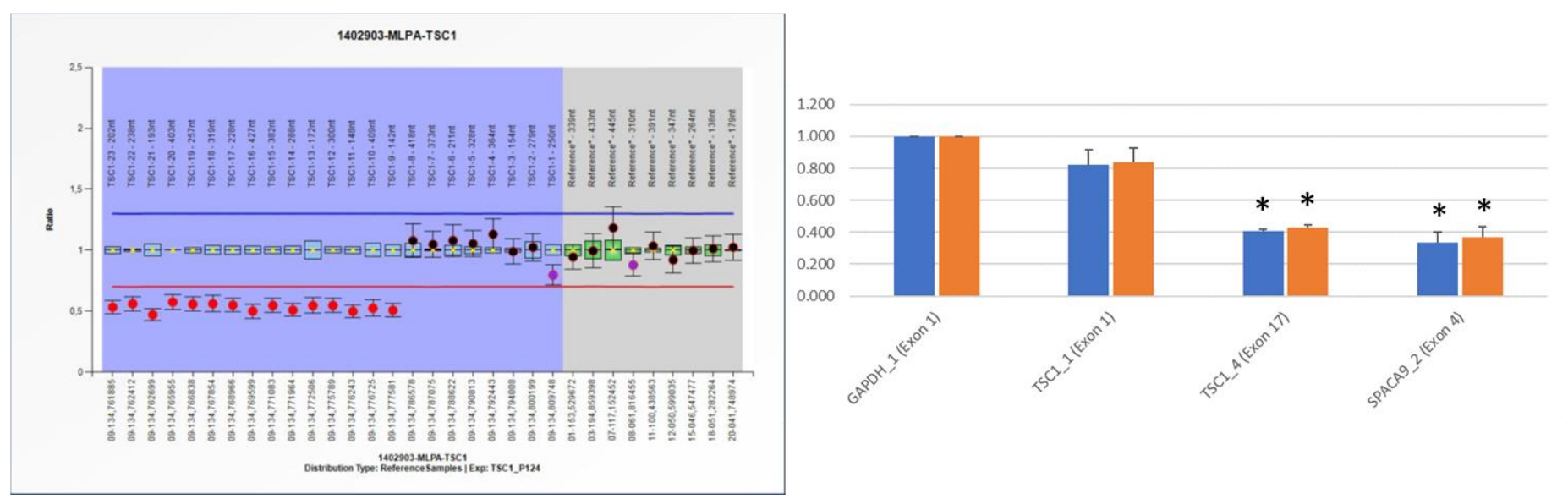

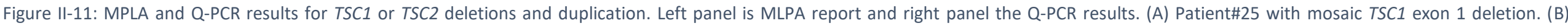

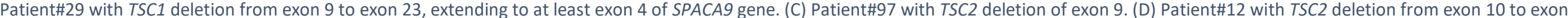

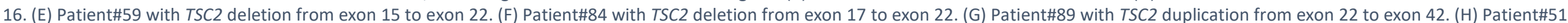

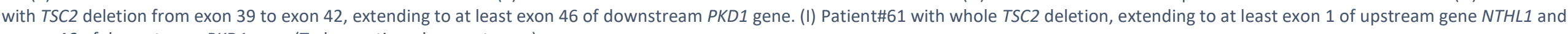
o exon 46 of downstream PKD1 gene (To be continued on next page). 


\section{C}

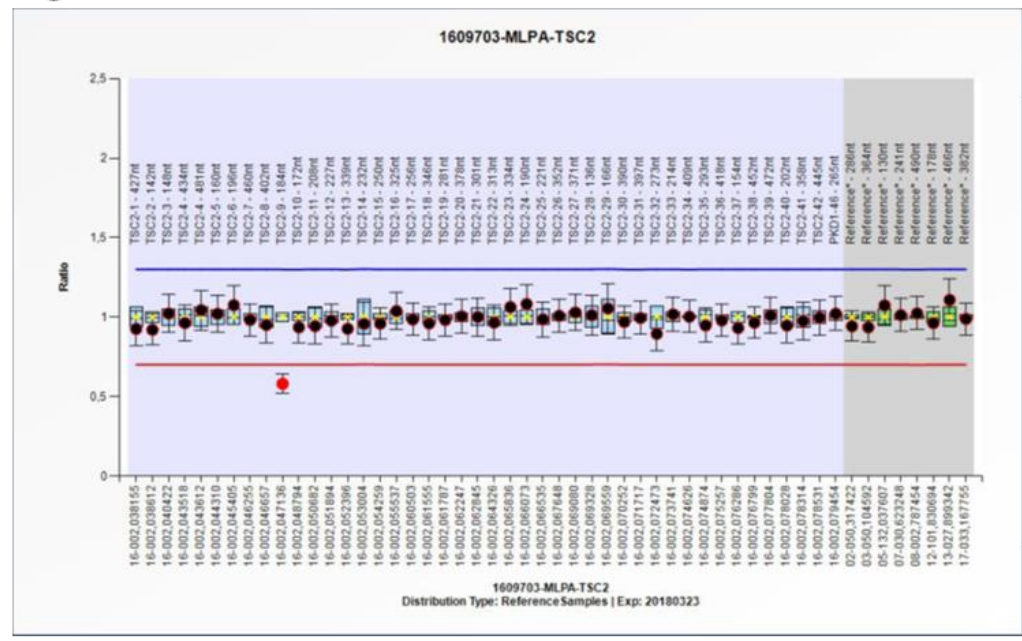

D

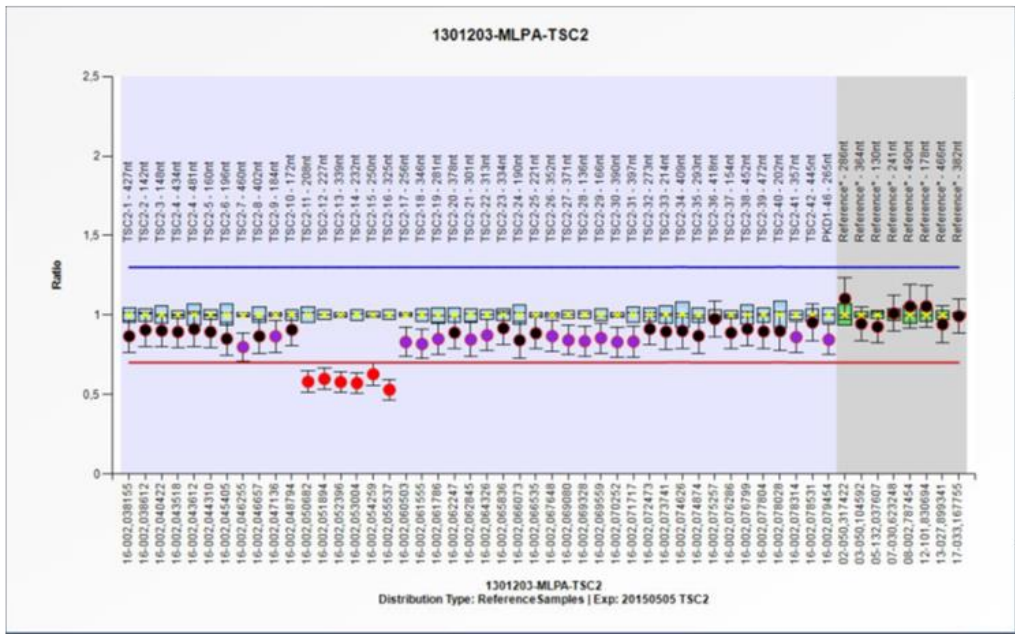

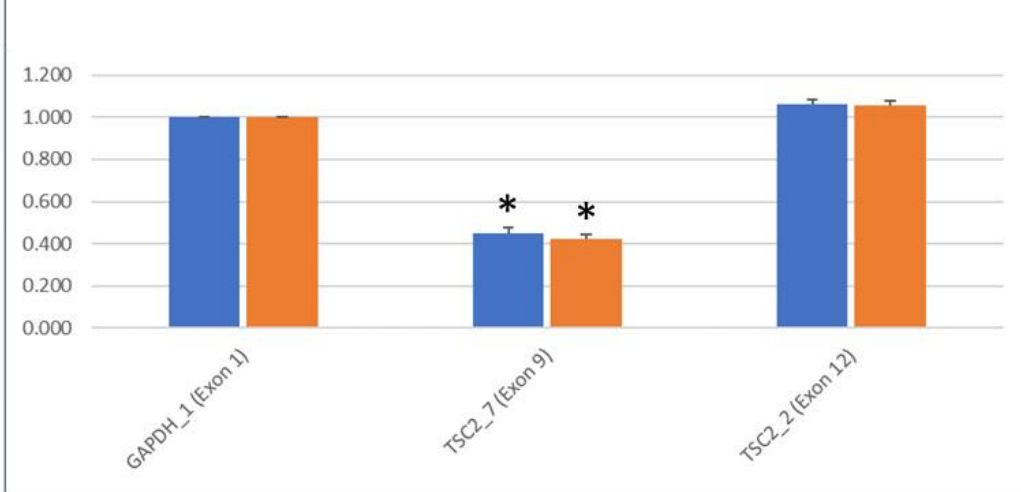

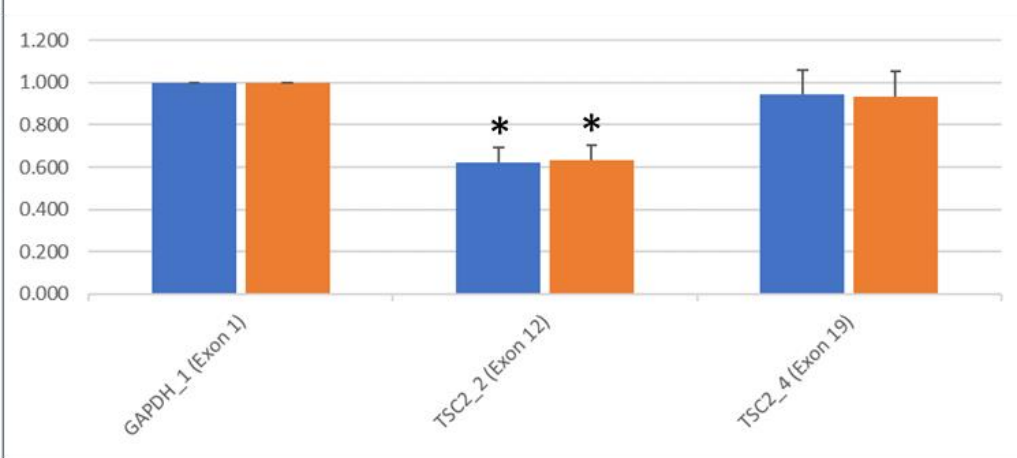




\section{E}
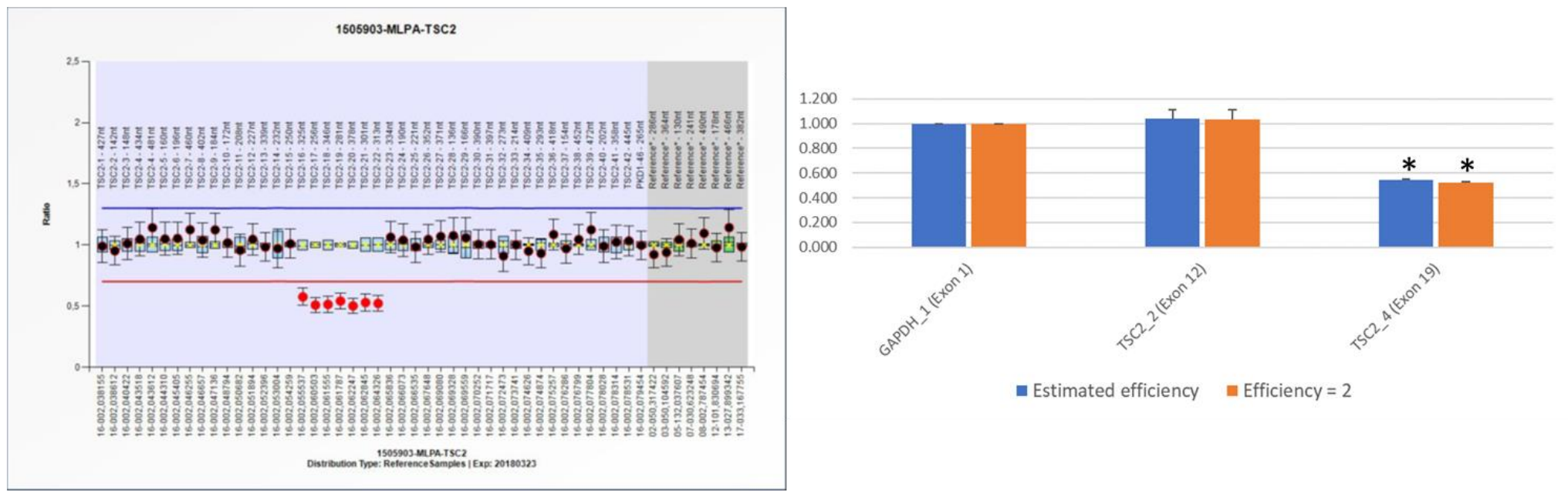

$\mathbf{F}$

1608403-MLPA-TSC
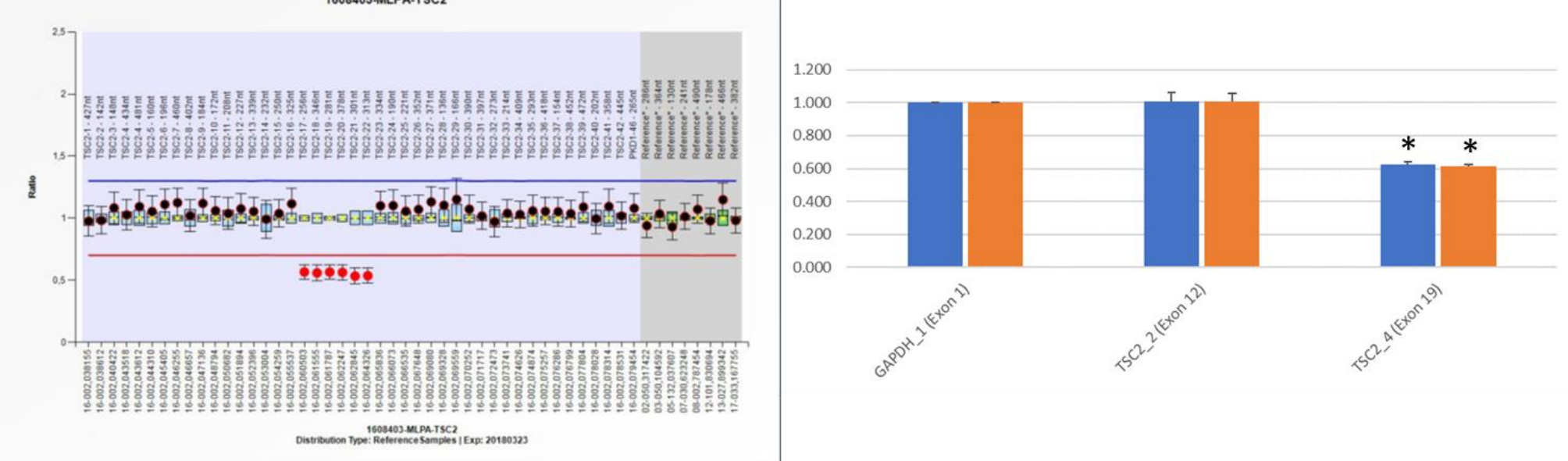

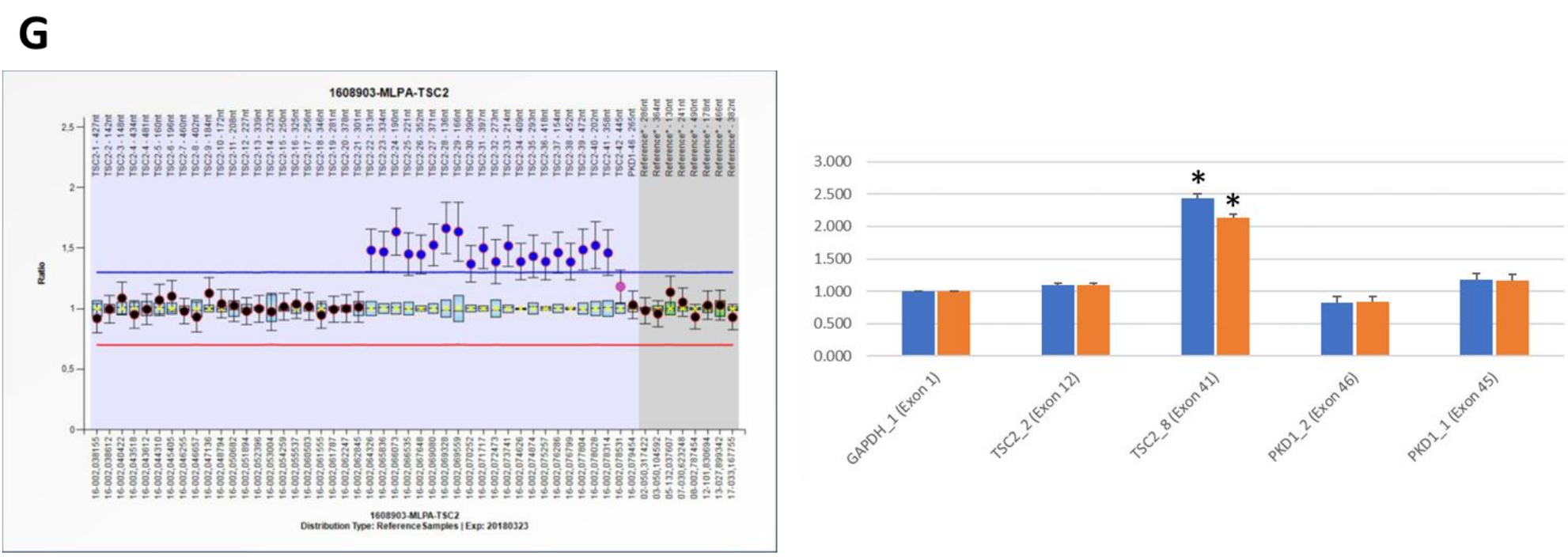

\section{H}
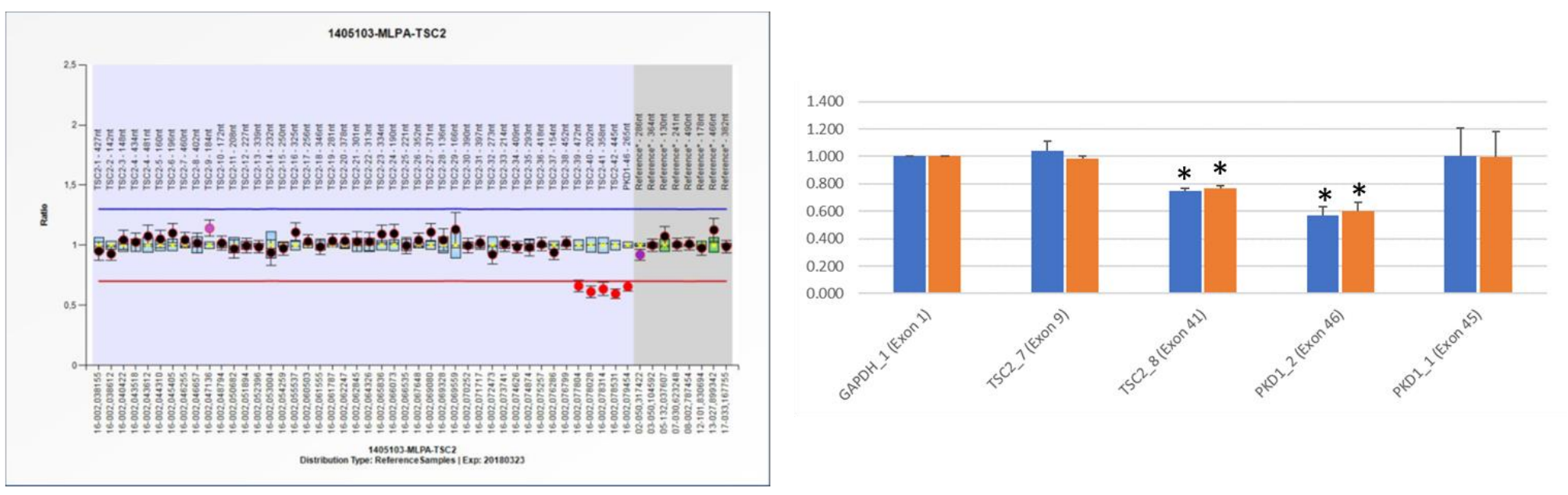

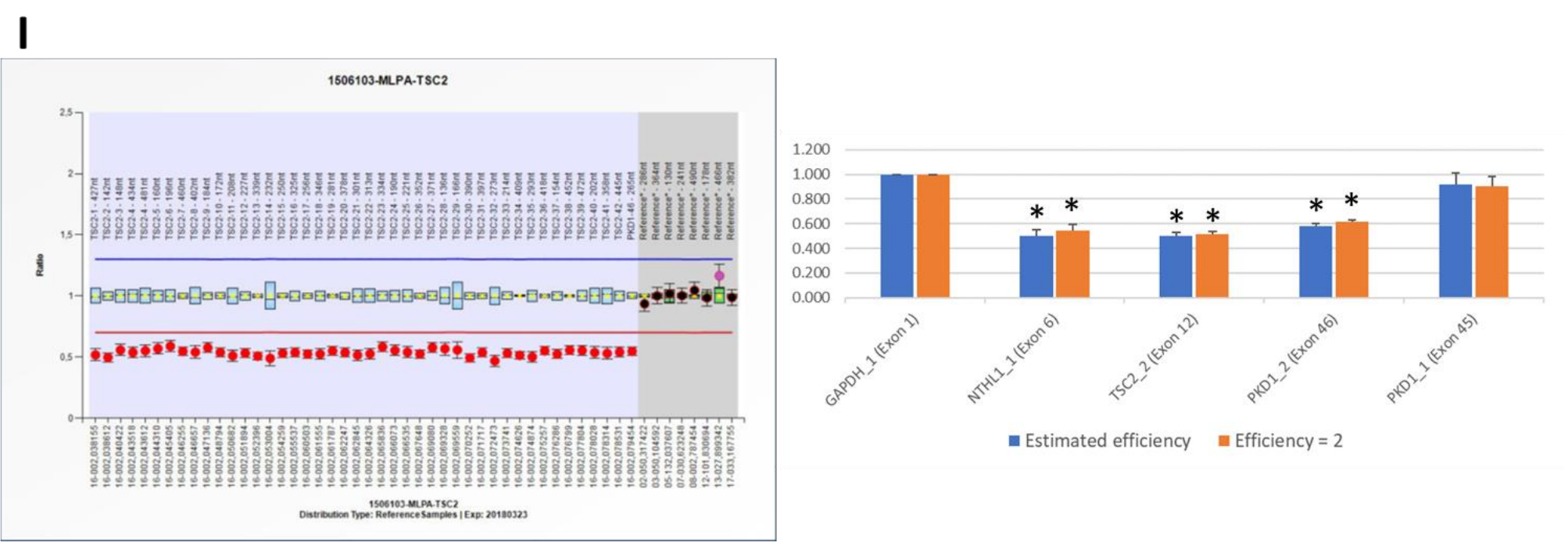
We attempted to identify the extension of the eight segmental deletions and the TSC2 duplication. GFI1B (Growth Factor-Independent 1B Transcription Repressor - NM_004188.6), a gene positioned in a headto-head fashion $34 \mathrm{~kb}$ upstream TSC1, was employed to investigate the boundary of patient \#25 mosaic deletion of TSC1 exon 1. GFIIB exon 1 deletion was ruled out by $Q-P C R(r q=0.93$; sem $=0.06 ; p=0.34$ ) (Figure II-11A). The long extension between TSC1 exon 1 and upstream GFI1B exon 1 (nearly $34 \mathrm{~Kb}$ ) or downstream TSC1 exon 2 (TSC1 intron 1 of $6 \mathrm{~Kb}$ ) impaired the identification of the breakpoints by PCR.

The extension of TSC1 deletion c.(737+1_738-1)_(*1+_?)del from patient \#29 was analyzed by Q-PCR. The SPACA9 (Sperm acrosome-associated 9 - NM_001316898.1) gene is approximately 2 kb downstream of TSC1 polyadenylation signal. Q-PCR of TSC1 3'-UTR ( $r q=0.49 ;$ sem $\left.=0.03 ; p=4.1 \times 10^{-2}\right)$ and of SPACA9 exon 4 $\left(r q=0.38 ;\right.$ sem $\left.=0.06 ; p=9.3 \times 10^{-3}\right)$ confirmed the deletion comprised both segments (Figure II-11B).

Four internal deletions have been detected by MLPA on TSC2 (Table II-8; patients \#97, 12, 59, and 84). Table II-8 indicates the expected locations of upstream and downstream breakpoints that generated those deletions.

Table II-8: TSC2 internal deletion breakpoint mapping

\begin{tabular}{|c|c|c|c|c|c|c|}
\hline \multirow{3}{*}{ فㄹㄹㄹ } & \multirow{3}{*}{ CNV } & \multirow{3}{*}{ Position } & \multicolumn{4}{|c|}{ Breakpoint location } \\
\hline & & & \multicolumn{2}{|c|}{ Expected } & \multicolumn{2}{|c|}{ Observed } \\
\hline & & & Upstream & Downstream & Upstream & Downstream \\
\hline 97 & c.(774+1_775-1)_(848+1_849-1)del & Exon 9 & Intron 8 & Intron 9 & Inc & Inc \\
\hline 12 & c. $(975+628) \_(1716+41)$ del & Exon 11-16 & Intron 10 & Intron 16 & Intron 10 & Intron 16 \\
\hline 59 & c.(1599+1_1600-1)_(2545+1_2546-1)del & Exon $16-22$ & Intron 15 & Intron 22 & Inc & Inc \\
\hline 84 & c.(1716+1_1717-1)_(2545+1_2546-1)del & Exon $17-22$ & Intron 16 & Intron 22 & Inc & Inc \\
\hline
\end{tabular}

Various forward and reverse PCR primers were designed to anneal respectively on upstream and downstream sites as an approach to sequence the junction of breakpoints. This strategy was successful to identify the breakpoints of TSC2 intragenic deletion c.(975+628)_(1716+41)del from patient \#12. Sequencing of PCR products amplified with primers annealing to introns 9 and 17 disclosed the deletion spans $6.2 \mathrm{~kb}$, from c. $975+627$ (intron 10) to c.1716+41 (intron 16). The breakpoint in intron 10 maps to an Alu repeat flanked by other Alu units, whereas the intron 16 breakpoint is $296 \mathrm{bp}$ upstream of a L1 repeat with three Alu repeats downstream. TSC2 intron 10 and intron 16 sequences, respectively 1,796 bp and 4,820 bp long, have only 11\% similarity. However, the 909-bp segment encompassing the short interspersed nuclear element (SINE) sequences within intron 10 and 16 breakpoints are 66\% identical (Figure II-12). A defined PCR product spanning the breakpoint junction was not obtained from DNA of patients \#97, 59 or 84 (Table II-8). 
A

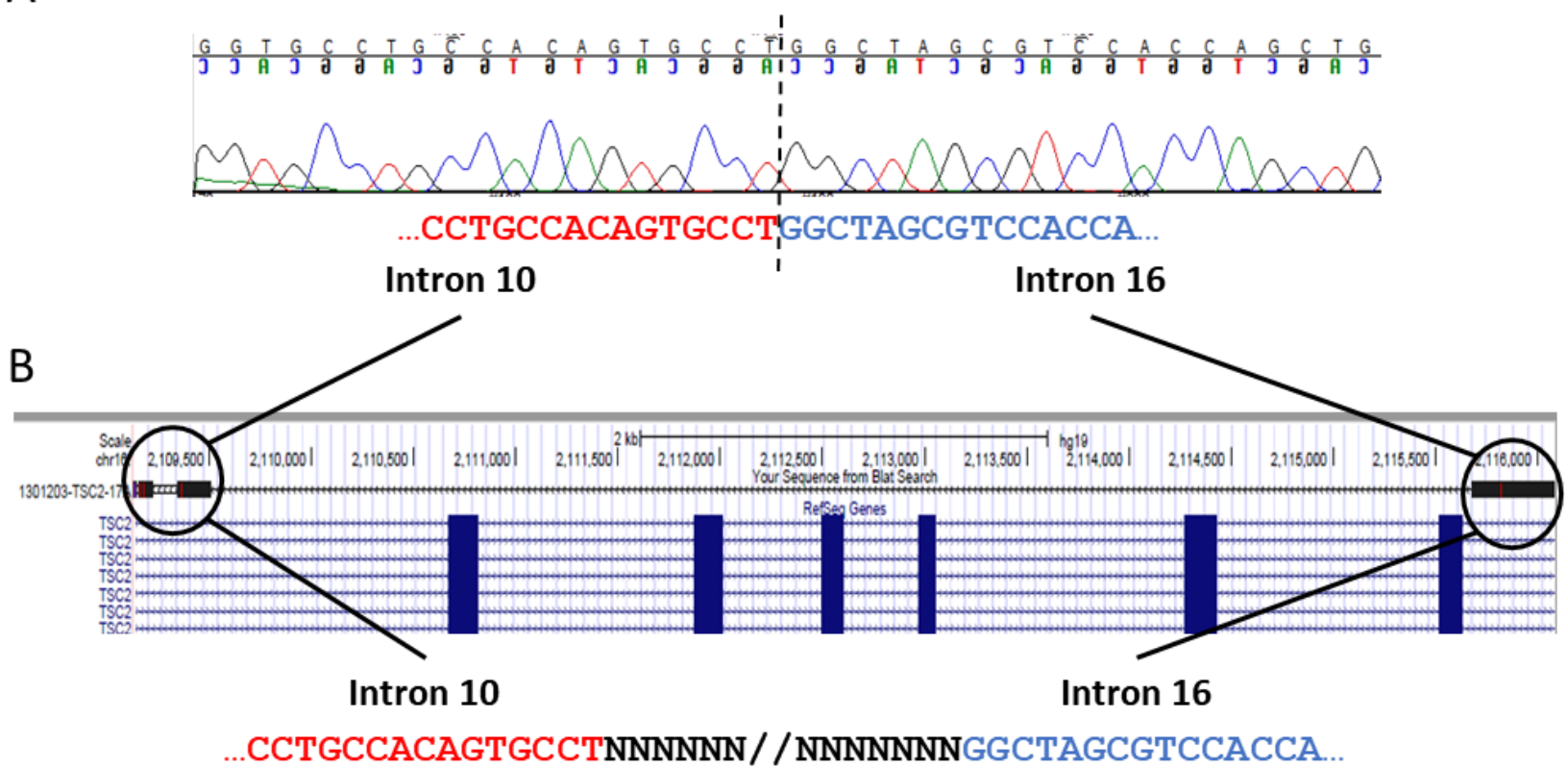

C

LINE/L1 class $\Rightarrow$ SINE/Alu class
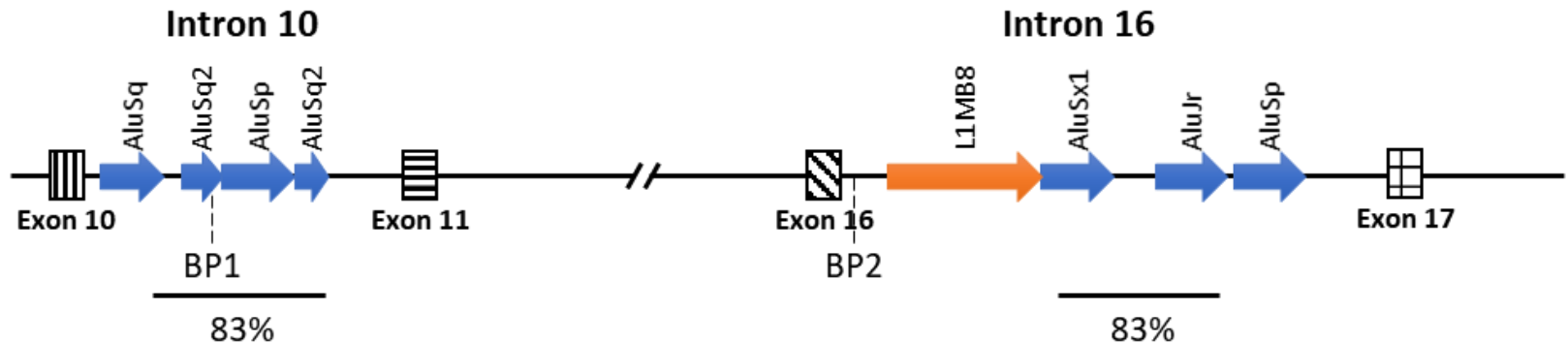

Figure II-12: Breakpoint mapping of the TSC2 intragenic deletion c.(975+_976-)_(1716+_1717-)del . (A) Sanger sequencing of the DNA from the patient with the intragenic TSC2 deletion c.(975+_976-)_(1716+_1717-)del. (B) UCSC genome browser mapping of the breakpoints in TSC2 introns 10 and 16. (C) Diagram illustrating the positions of SINE/Alu and LINE/L1 repeat sequences in TSC2 introns 10 and 16 , respectively within and adjacent to each breakpoint. The regions of $83 \%$ similarity between introns are indicated.

One segmental duplication and two additional deletions were identified in the TSC2 gene (Table II-7 and Figure II-11). TSC2 is tail-to-tail to PKD1 (Polycystic Kidney Disease 1 gene, NM_001009944). In other to evaluate if the CNV spanned through PKD1, its most 3' exons, 45 and 46, were tested by Q-PCR in those three cases. In the case of c.(2355+1_2356-1)_(*102_?)dup (Patient \#89), neither exon 45 or 46 was duplicated (exon 45: $r q=1.18$, sem $=0.1 ; p=0.14 ;$ exon 46: $r q=0.83$; sem $=0.09 ; p=0.19$ ) (Figure II-11G).

Patient \#51 TSC2 deletion of exon 39 (c.(5068+1_5069-1)_(*102_?)del) extended through PKD1 exon 46 , as validated by $Q-P C R(r q=0.57$; sem $=0.06 ; p=0.02)$, though exon 45 was preserved $(r q=1.0$; sem $=0.2$; $p=0.99)($ Figure II-11H). Hence, upstream and downstream breakpoints should be respectively at TSC2 intron 38 and PKD1 intron 45. Similarly, the deletion of full TSC2 gene c.(?_-30)_(*102_?)del (patient \#61) encompassed $P K D 1$ exon 46 as detected by $\mathrm{Q}-\mathrm{PCR}\left(\mathrm{rq}=0.58\right.$; sem $\left.=0.02 ; \mathrm{p}=2.2 \times 10^{-3}\right)$, preserving exon 45 ( $\mathrm{Q}$ - 
PCR; $r q=0.92 ;$ sem $=0.1 ; p=0.42$ ). The NTHL1 (Endonuclease III-Like 1, MIM\#602656) lies upstream TSC2 in a tail-to-head fashion. Q-PCR of NTHL1 exon $6\left(r q=0.50\right.$; sem $\left.=0.05 ; p=8.6 \times 10^{-3}\right)$ disclosed that the deletion affected the $3^{\prime}$ end of this gene as well. 
In summary, Figure II-13 and 16 illustrate the eight CNV identified as TSC1 (1) or TSC2 (7) pathogenic alterations, as well as the mosaic TSC1 exon 1 deletion, which was considered an alteration of uncertain significance. Their estimated extension is presented according to the gene exon location.

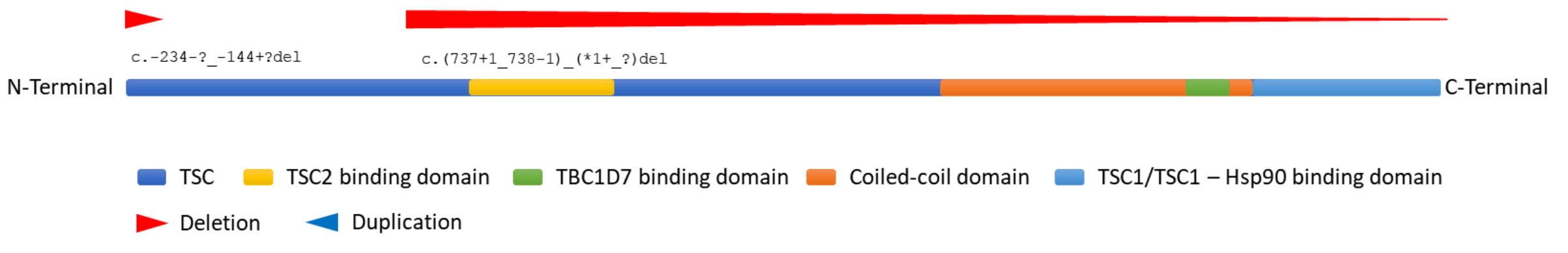

Figure II-13: Schematic representation of TSC1 and the segmental deletion c.-234-?_-144+?del of exon 1 on patient\#25 c.(737+1_738-1)_(*1+_?)del from exon 9 to exon 23 fond on patient\#29. 


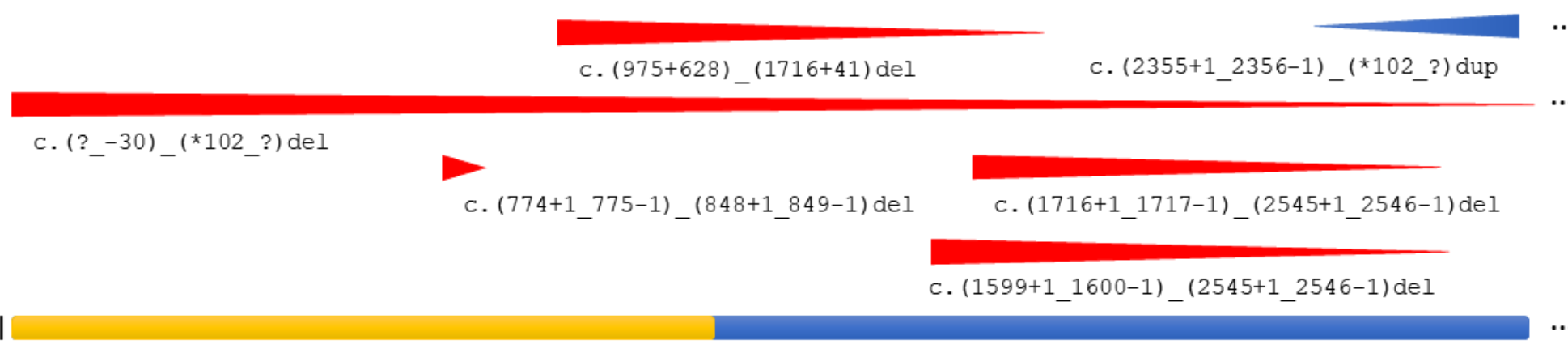

$\mathrm{N}$-Terminal

c. $\left(1599+1 \_1600-1\right) \_\left(2545+1 \_2546-1\right)$ del

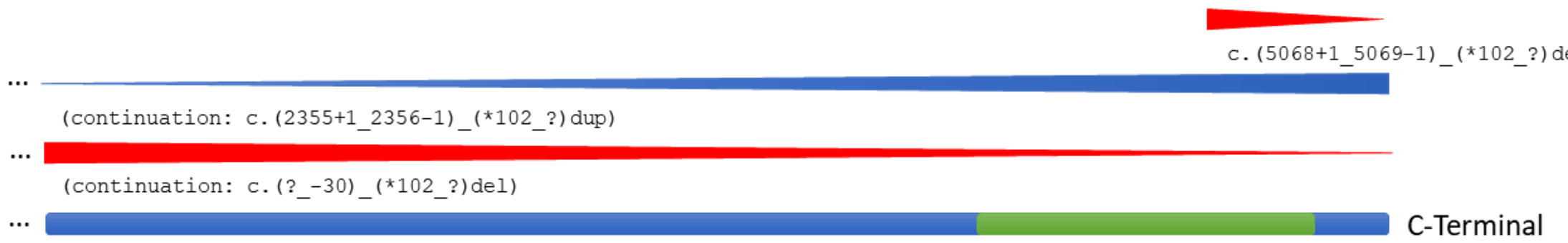

\section{$\square$ TSC2 $\square$ TSC1 biding domain $\square$ GAP domain \\ Deletion Duplication}

Figure II-14: Schematic representation of TSC2 and the segmental deletion c.(975+628)_(1716+41)del from exon 11 to exon 16, c.(5068+1_5069-1)_*102_?)del from exon 39 to exon 42, c.(1599+1_16001)_(2545+1_2546-1)del from exon 16 to exon 22, c.(??-30)_*102_?)del whole TSC2, c.(1716+1_1717-1)_(2545+1_2546-1)del from exon 17 to exon 22, c.(774+1_775-1)_(848+1_849-1)del from exon 22 to exon 42, and duplication c.(2355+1_2356-1)_(*102_?)dup form exon 22 to 42. 


\section{E. Single nucleotides variants}

Finally, we identified 176 distinct, single nucleotide variants (SNV; frequency higher than 0.01) without clinical significance, 48 on TSC1 and 128 on TSC2. Of those, 53 variants were identified within exonic segments (Supplementary Table II-11) and 113 in introns (Supplementary Table II-12). Nineteen variants were not present in 1000 Genomes, dbSNP and COSMIC data banks, and occurred in patients with pathogenic variants (Supplementary Table II-11 and 12).

NMI patients presented ten rare DNA alterations that classified as VUS, three in TSC1 (c.-234-?_144+?del, c.738-197T>A and c. ${ }^{* 1 G>A}$ ), and in TSC2: c.1119G>A (p.=), c.1443G>T (p.Glu481Asp), c.1663G>C (p.Ala555Pro), c.4493G>T (p.Ser1498Ile), c.5051C>T (p.Ser1684Phe), c.4005+153T>A and c.*44_*45insGTCA (Table II-4 and 7). 


\section{Discussion}

We analyzed DNA from 117 patients with definite clinical diagnosis of TSC (Northrup, Krueger et al. 2013). They were from the states of São Paulo (SP), Paraná (PR) or Santa Catarina (SC), in the South and Southeast regions of Brazil; and clinically followed up at three large, tertiary referral University hospitals (HSCM-SP, GRAACC-SP, and HC-UFPR). The clinical evaluation of these patients has been the subject of other graduate research studies (Juliana P Almeida Master's dissertation - Hospital Santa Casa de Misericórdia, 2016, Laís Masulk Cardoso, Master's dissertation - Complexo de Hospital de Clínicas da Universidade Federal do Paraná, Laís Masulk ; and Nasjla Saba, unpublished), and will thus not be discussed here. The vast majority of samples has been first assessed by Sanger sequencing $(\mathrm{N}=116)$, whereas one sample was initially analyzed by NGS. Pathogenic DNA variants identified by NGS have been confirmed by Sanger sequencing. MLPA was carried out in a subset of patient DNA ( $N=38)$, and any altered result further assessed by Q-PCR.

We report a mutation detection rate of $87.2 \%$ (102/117), which is similar to previous reports that sequenced both TSC1 and TSC2 coding sequence and intronic boundaries, and assessed exonic copy number variation (Jones, Shyamsundar et al. 1999, Dabora, Jozwiak et al. 2001, Rendtorff, Bjerregaard et al. 2005, Sancak, Nellist et al. 2005, Hung, Su et al. 2006, Au, Williams et al. 2007). Despite the high number of distinct TSC-causing mutations already described worldwide in both TSC1 and TSC2 genes (LOVD-TSC1, LOVD-TSC2), we report 41 novel pathogenic DNA variants (Table II-4 and 5) as well as 19 novel SNV (Supplementary Table II-11 and 12).

It has been reported that patients with mutation identified, either on TSC1 or TSC2 genes, are generally more severely affected than patients with NMI, who present a lower incidence of brain findings on imaging studies, neurological features, renal findings and lower incidence of seizures than those with TSC1 or TSC2 mutations (Camposano, Greenberg et al. 2009). Our mutation detection rate by currently standard sequencing approaches lies in the average rate as other studies. Ninety-eight patients were from Neurological services from two tertiary referral hospitals (HC-UFPR and HSCM, SP), and the remaining 19 patients were from the Oncology service of another tertiary referral University hospital (UNIFESP, São Paulo, SP). Tertiary referral hospitals tend to retain the most severe cases.

In regard to TSC de novo cases, when patients with TSC2 mutations are compared to those with TSC1 mutations, they present higher mean number of cerebral cortical tubers and subependymal nodules, mean grade of kidney angiomyolipomas, as well as frequency of moderate/severe cases of intellectual disability and retinal hamartomas (Dabora, Franz et al. 2011). Accordingly, Sancak, Nellist et al. (2005) and Au, Williams et al. (2007) reported intellectual disability, angiomyolipoma and retinal phacoma more frequently among TSC patients with TSC2 than TSC1 mutations. As mentioned, all patients from our cohort came from tertiary referral hospitals, which tend to assist more severe cases. This could justify the higher numbers of TSC2 
pathogenic variants observed by us. However, if we estimate this ratio specifically for each hospital, we observe that $71.4 \%(60 / 84)$ of all TSC2 pathogenic variants in the present study are from HC-UFPR while TSC1/TSC2 ratio from HSCM-SP is nearly 1:1.5 (Table II-3). At enrolment in this study, familial cases have been informed based solely on parental report. Among the 102 cases with pathogenic alteration identified, familial cases are expected to be 30\% of all TSC cases (Jones, Shyamsundar et al. 1999, Dabora, Jozwiak et al. 2001, $\mathrm{Au}$, Williams et al. 2007). It remains to be evaluated if more familial cases could be part of the HSCM-SP cohort of 23 patients, what could explain the 1:1.5 TSC1:TSC2 partition ratio for pathogenic DNA alterations.

Frameshift and nonsense variants were the alteration types most commonly observed among TSC1 and TSC2 pathogenic DNA alterations. Altogether nonsense and frameshifting pathogenic variants corresponded to $94.4 \%(17 / 18)$ and 50\% (42/84) of all genetic alterations, respectively for TSC1 and TSC2 genes. In our study, missense, in-frame deletions and splicing DNA variants have been detected only in the TSC2 gene. Segmental gene deletions and duplications comprehended 7.8\% (8/102) of all pathogenic DNA alterations, 5.5\% (1/18) and 8.3\% (7/84) respectively for TSC1 and TSC2. Of note, 2.5\% of TSC patients with a mutation identified had TSC2 deletions extending through PKD1 gene, defining cases with the contiguous gene deletion syndrome (Martignoni, Bonetti et al. 2002). Our data are similar to another Brazilian study (Rosset, Vairo et al. 2017) as well as to reports from many countries (Rendtorff, Bjerregaard et al. 2005, Hung, Su et al. 2006, Au, Williams et al. 2007, Kozlowski, Roberts et al. 2007, Sasongko, Wataya-Kaneda et al. 2008, Chopra, Lawson et al. 2011, Niida, Wakisaka et al. 2013). Because our study does not aim to estimate the frequency of TSC in Brazil, we should not compare our data to previous reports that reported on the lack of ethnic or geographical bias of TSC distribution (Wiederholt, Gomez et al. 1985, Sampson, Scahill et al. 1989, Osborne, Fryer et al. 1991, O'Callaghan, Shiell et al. 1998).

\section{A. $\quad$ TSC2 missense and in-frame deletion effect on mTOR}

Missense DNA variants cause amino acid substitution in the encoded protein. As missense variants are not considered a priori a loss-of-function variant, the interpretation of their clinical significance is not straight forward, and relies on several bioinformatics tools, although based on common biological aspects. PlolyPhen2 involves comparison of biochemical and genetic properties between the wild-type (ancestral, normal) allele and those of the mutant (derived, disease-causing) allele (Adzhubei, Schmidt et al. 2010). Mutation Taster integrates information from different biomedical databases and uses established analysis tools, comprising evolutionary conservation, splice-site changes, loss of protein features and changes that might affect the amount of mRNA (Schwarz, Rodelsperger et al. 2010). SIFT and PROVEAN predict whether a protein sequence variation affects protein function (Ng and Henikoff 2003, Choi and Chan 2015).

Among 20 missense variants and five rare in-frame deletion variants identified, at least two in silico bioinformatics tools predicted damaging effects, except for c.2167A>G (p.lle723Val) for which Mutation Taster 
was the only one to predict a deleterious output. Moreover, splicing analysis using Human Splicing Finder also predicted for almost all variants submitted to analysis the potential to disrupt splicing. The only exceptions were c.1663G>C (p.Ala555Pro), c.1831C>T (p.Arg611Trp) and c.2167A>G (p.lle723Val).

Our in silico predictions agreed with previous functional assessments for variants c.1831C>T (p.Arg611Trp), c.1832G>A (p.Arg611GIn), c.5227C>T (p.Arg1743Trp) and c.5228G>A (p.Arg1743GIn) (Hoogeveen-Westerveld, Ekong et al. 2013). However, TSC2 variants c.499T>C (p.Trp167Arg) and c.1793A>G (p.Tyr598Cis) had been predicted as pathogenic by all in silico analysis, but disclosed no inactivating effects on mTOR by the functional assessment. Moreover, our functional assay disagrees with in silico prediction for c.52C>G (p.Leu18Val) and c.499T>C (p.Trp167Arg), which classified as deleterious but did not activate the mTORC1 pathway (Figure II-7). Finally, c.4493G>T (p.Ser1498Ile), c.4909_4911delinsGAC (p.Lys1637Asp), c.4919A>G (p.His1640Arg) and c.5227C>G (p.Arg1743Gly) were predicted to be deleterious by all in silico analysis, supported by our functional assessment (Figure II-7). The remaining variants are under investigation.

Well-established in vitro or in vivo functional assays supportive of a damaging effect on the gene product or pathway have been classified as a strong criterion for pathogenicity classification of missense DNA variants (Richards, Aziz et al. (2015), Supplementary Table II-9).Based on the recognized repressive role of TSC1/2 complex on mTORC1 kinase activity, a functional assay has been developed (Hoogeveen-Westerveld, Wentink et al. 2011, Hoogeveen-Westerveld, Ekong et al. 2012, Hoogeveen-Westerveld, Ekong et al. 2013, Kwiatkowski, Palmer et al. 2015) and further improved (Dufner-Almeida, L. G. et. al. submitted). It relies on reporting the phosphorylation status of S6K-Thr ${ }^{389}$, a target of mTORC1 (Goncharova, Goncharov et al. 2002, Tee, Fingar et al. 2002). We employed the mTORC1 activity-based assay to functionally assess five TSC2 missense variants, two TSC2 in-frame deletions, and one splicing variant (Figure II-7). A genetically modified HEK293T cell line (3H9-1B1) having both TSC1 and TSC2 genes fully inactivated by CRISPR/Cas9 (Figure II-7; Dufner-Almeida et al., manuscript submitted) was used in the assay. For each assay, the 3H9-1B1 cell line was transfected with three different full-length cDNA clones (WT or mutant TSC2, WT TSC1, and WT RPS6KB1 $S 6 K)$. This additional step leads to higher efficiency in reporting S6K-Thr ${ }^{389}$ phosphorylation due to higher stability of overexpressed TSC1/2 complex than its endogenous levels (Dufner-Almeida, L. G. et. al. submitted). TSC1 and S6K protein levels were not affected by the overexpression of any TSC2 variant tested in the present study (Figure II-7C and D). TSC2 levels were reduced by Ser1498Ile and 1608del variants (Figure II-7B) with no effect on TSC1 and S6K. Because all three proteins, TSC1, TSC2 and S6K, are co-overexpressed, TSC2 levels are unlikely to be solely affected by contaminants from transfection. Hence, variants Ser1498lle and 1608del may render TSC2 unstable, possibly due to incorrect folding and faster degradation than wild-type TSC2. Moreover, as both variants are outside the TSC1-binding domain, the TSC1-TSC2 interaction should not be destabilized (Chong-Kopera, Inoki et al. 2006), thus preserving TSC1 levels. 
A

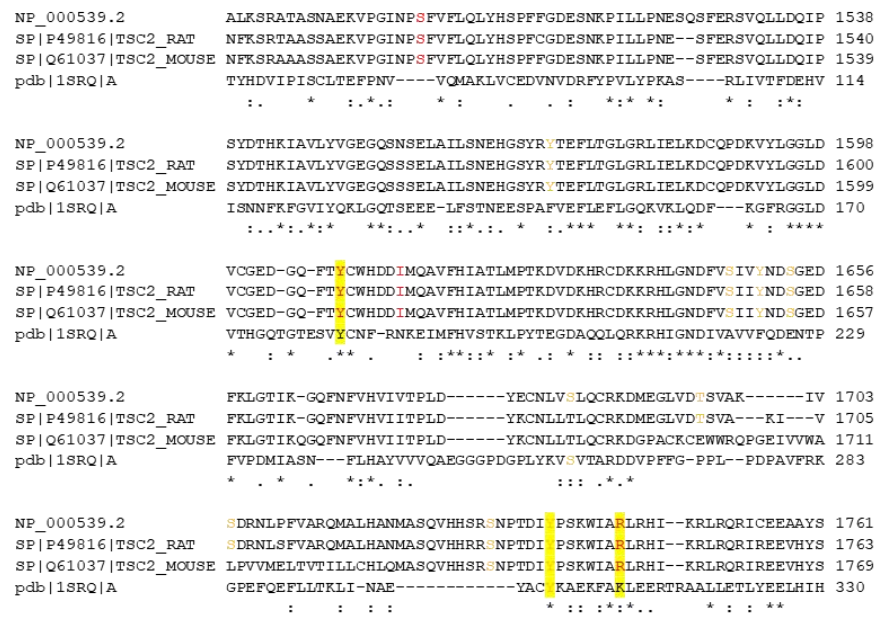

B

$\mathrm{C}$
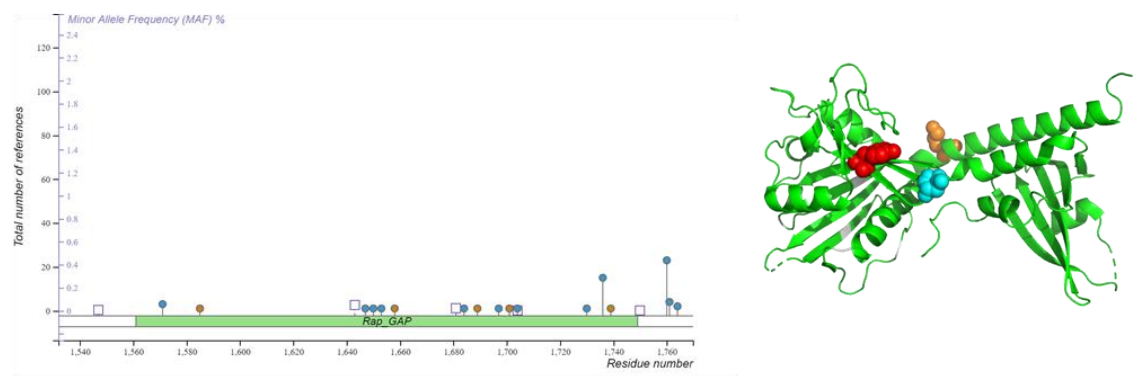

D

$\mathrm{E}$
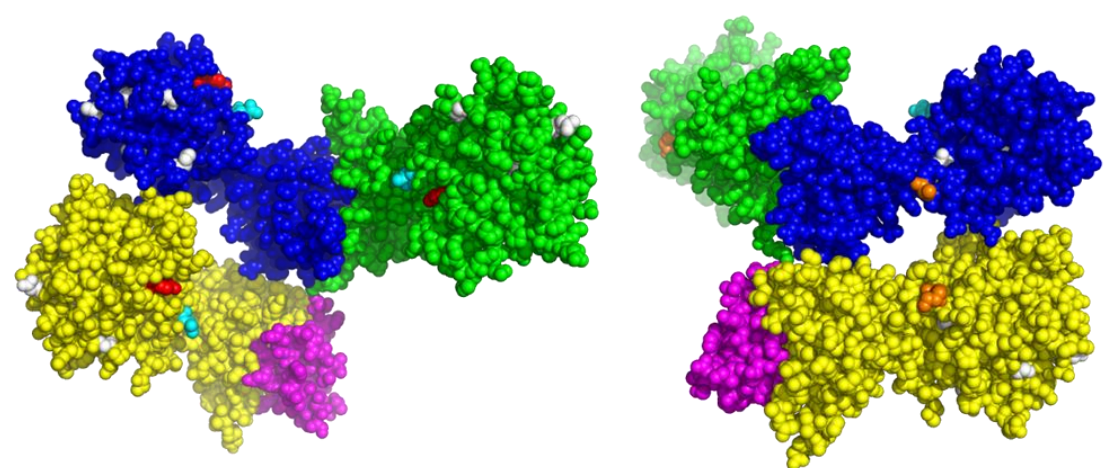

Figure II-15: Global alignment of TSC2, phosphorylation site of GAP Domain and positioning of TSC2:p.Tyr1608del, p.lle1614del and p.Arg1743Gly variants on predicted Homo sapiens GAP domain structure (PDB: 1SRQ). (A) Global alignment of human (NP_000539.2), rat (P49816), mouse (Q61037) TSC2 C-terminal and Rap1GAP catalytic domain (Homo sapiens, pdb|1SRQ|A) (Daumke et al., 2004). Red letters represent amino acids conserved among human, rat and mouse, and red letters highlighted in yellow represent the a mino acids conserved between the three organisms and the predicted 1 SRQ sequence. Below the alignment, asterisk $\left({ }^{*}\right)$ indicates positions which have a single, fully conserved residue, colon (:) indicates conservation between groups of strongly similar properties (scoring > 0.5 in the Gonnet PAM 250 matrix), period (.) indicates conservation between groups of weakly similar properties (scoring $=<0.5$ in the Gonnet PAM 250 matrix) and none indicates none conservation between groups. (B) Graphic representation of phosphorylation sites on C-Terminal domain of TSC2. (C) Cartoon representation of tertiary structure of 1SRQ|A generated with PyMOL software. Red sphere represents the TSC2:p.Tyr1608del variant, cyan sphere represent TSC2:p.lle1614del variant, orange sphere represent TSC2:p.Arg1743Gly variant and white sphere the phosphorylated residues. (D) and (E), respectively front and back schematic representation of 1SRQ crystal structure of the Rap1GAP catalytic domain (Homo sapiens) and the position of TSC2:p.Tyr1608del, p.lle1614del and p.Arg1743Gly variants.

We identified five variants that significantly increased $\mathrm{S} 6 \mathrm{~K}-\mathrm{Thr}^{389} / \mathrm{S} 6 \mathrm{~K}$ ratios, a result from reduced TSC1-TSC2 inhibition on mTORC1 activity (Tee, Fingar et al. 2002, Inoki, Li et al. 2003, Zhang, Cicchetti et al. 
2003, Riemenschneider, Betensky et al. 2006). Two missense variants (His1640Arg and Arg1743Gly), one inframe exon skipping due to splice variant (1044del50), and two in-frame deletions (Tyr1608del and Ile1614del) were thus classified as pathogenic (Figure II-7). TSC-causing missense variants have been described more often in the coding sequence for TSC1 or TSC2 domains, notably TSC2 GAP domain and TSC2/TSC1-interacting domain, such as Arg611GIn in the latter (Nellist, Sancak et al. 2005, Hoogeveen-Westerveld, van Unen et al. 2012). The missense Arg1743Gly variant and the two in-frame deletions (1608del and 1614del) locate within TSC2 GAP domain. Residues Ile1498, Thy1608, lle1614 and Arg1743 are conserved among human, rat and mouse TSC2 (Figure II-15A). None of those is close to phosphorylation sites (Figure II-15B). However, analysis of TSC2 GAP domain (pdb:1SRQ) by pymol shows that Thy1608 and lle1614 are close and both are towards the inner face of the structure, while the Arg1743 locates outside the domain folding and faces a probable catalytic pore (Figure II-15D and E). The spatial prediction of each variant and the possible function on activity of TSC2 GAP together with functional assessment (Figure II-7) suggest these residues are important for the structural stability of TSC2 GAP domain.

In two other cases (Leu18Val and Trp167Arg), the S6K-Thr $389 /$ S6K ratios were not significantly different from wild-type TSC2. The variants then classified as non-pathogenic. Variant c.499T>C (p.Trp167Arg) has been detected in an individual with a nonsense mutation. The functional assay corroborates it is a benign substitution. The missense Trp167Arg is conserved among human, rat and mouse TSC2 (Figure II-16A) and are not close to phosphorylation site (Figure II-16B). Moreover, Trp167Arg variant are located within alpha-helix (Figure II-16C) but embedded inside the predicted TSC1 binding domain (pdb|5HIU, Figure II-16D) of Chaetomium thermophilum. The spatial prediction and functional assessment suggests these residues are not important for TSC1/2 complex. 
A

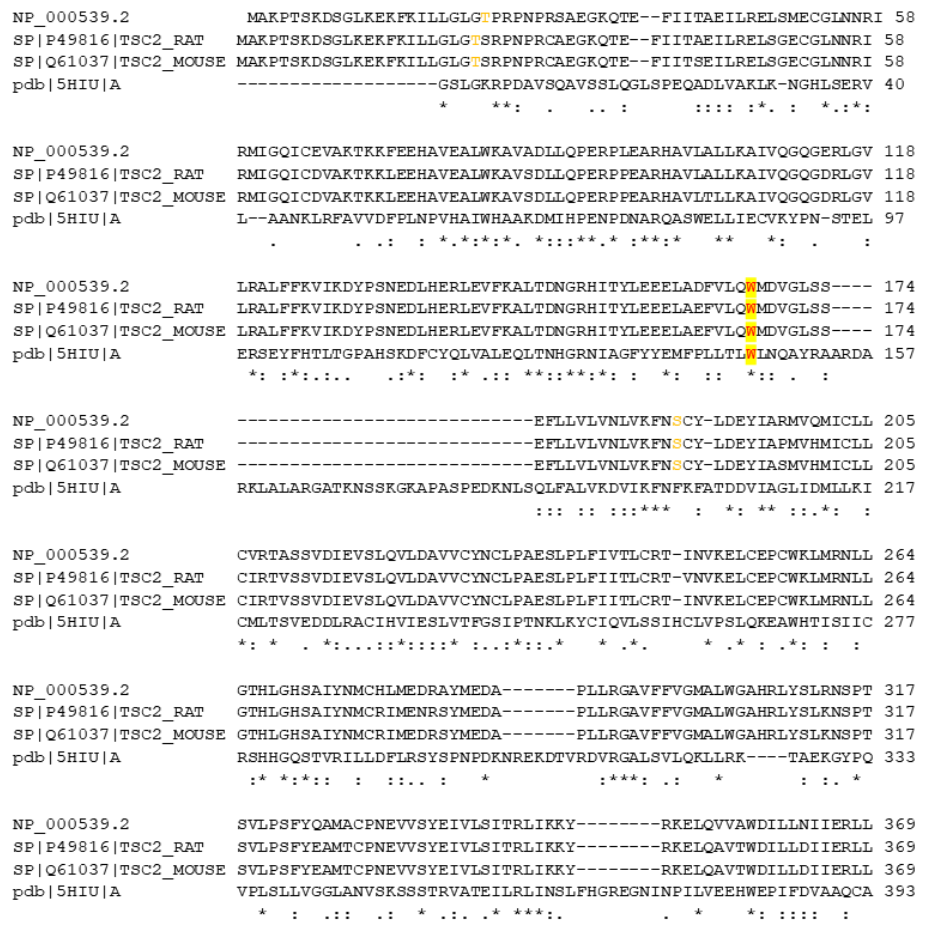

B

C

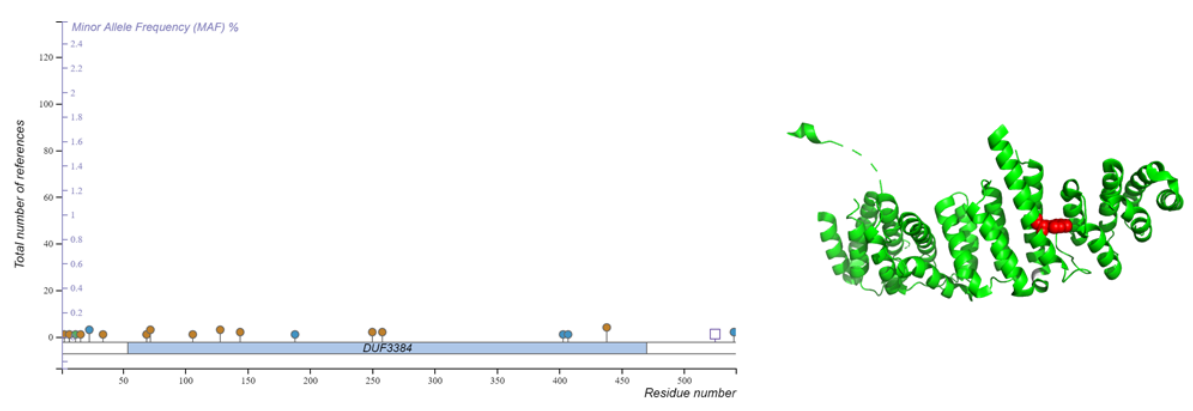

D

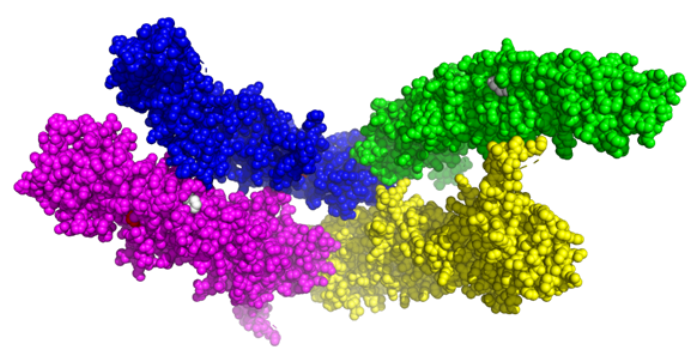

Figure II-16: Global alignment of TSC2, phosphorylation site of TSC1 binding domain and positioning of TSC2:p.Trp167Arg variant on predicted Chaetomium thermophilum TSC1 binding domain structure (PDB:5HIU). (A) Global alignment of human (NP_000539.2), rat (P49816), mouse (Q61037) TSC2 N-terminal and TSC1 binding domain (Chaetomium thermophilum, pdb|5HIU|A) (Zech, R., 2016). Red letters represent amino acids conserved among human, rat and mouse, and red letters highlighted in yellow represent the amino acids conserved between the three organisms and the predicted $5 \mathrm{HIU}$ sequence. Below the alignment, asterisk $\left({ }^{*}\right)$ indicates positions which have a single, fully conserved residue, colon (:) indicates conservation between groups of strongly similar properties (scoring $>0.5$ in the Gonnet PAM 250 matrix), period (.) indicates conservation between groups of weakly similar properties (scoring $=<0.5$ in the Gonnet PAM 250 matrix) and none indicates none conservation between groups. (B) Graphic representation of phosphorylation sites on N-Terminal domain of TSC2. (C) Cartoon representation of tertiary structure of 5HIU|A generated with PyMOL software. Red sphere represents the TSC2:p.Tyr1608del variant, cyan sphere represent TSC2:p.Trp167Arg and white sphere the phosphorylated residues. (D) Representation of 5HIU crystal structure of the TSC1 binding domain (Chaetomium thermophilum) and the position of TSC2:p.Trp167Arg. 


\section{B. 'Miscalled' missense DNA variants?}

One result of the mTOR-based functional assay, c.4493G>T (p.Ser1498/le), disclosed S6K-Thr ${ }^{389} / \mathrm{S} 6 \mathrm{~K}$ ratio similar to both, wild-type and Arg611GIn TSC2. This borderline result led us to consider c.4493G $>$ T with uncertain significance. As they did not significantly affect S6K phosphorylation (Figure II-7), we further evaluated the potential of c.4493G>T (p.Ser1498Ile) to affect pre-mRNA splicing. This substitution of unclear significance is on the last nucleotide of exon 34. Nucleotide substitutions, as in putative missense and synonymous variants, may lead to splicing abnormalities if they abolish exonic splicing enhancers (ESE) or silencers (ESS) or activate cryptic exon splice sites (reviewed by Dufner-Almeida et al. 2018). Variant c.4493G>T (p.Ser1498Ile) was predicted by ALAMUT to disrupt splice site (Supplementary Figure II-2). ALAMUT employs different softwares to predict effect on pre-mRNA splicing. Acescan2 identifying candidate cis-elements in alternative and constitutive splicing in mammalian exons and within the flanking introns. SpliceAid2 searches for motifs versus a database of experimentally assessed target RNA sequences, that can predict the effect of the DNA mutations at the level of the target sequences of the RNA-binding proteins that determine the pattern of mRNA splicing. Finally, HSF combines algorithms based on PWM matrices, Maximum Entropy principle or Motif Comparison to identify and predict the variant effect on splicing RNA motifs.

To functionally assess the effect of c.4493G >T on the splicing of TSC2 exon 34, the mini-gene strategy was employed by cloning and expressing the genomic DNA segment from exon 33 through exon 35 . The internal exon (34) was interrogated to contain an element that regulates its splicing. The expression of the mini-gene in transfected eukaryotic cells produces the processed mRNA. If internal transcribed elements of the mini-gene are recognized by the cell nuclear proteome, differential splicing may take place. We did not obtain evidence for an effect of the c.4493G>T variant on splicing on HEK293 cells. However, as transcription output depends on the combinatorial effect of trans-acting elements, splicing variants may have as well different effects according to tissue.

A 4-bp deletion (TSC2:c.1361+54_1361+57del) from one NMI patient was not identified on 1000 Genomes, but twice on dbSNP (rs137854304) and once on ClinVar (ID 49694) without clinical significance reported. The four bases are embedded within a putative intronic splicing enhancer potentially recognized by a trans-acting protein factor CUGBP1/CELF1 that is widely expressed in over 20 human tissues (Fagerberg, Hallstrom et al. 2014). Tissue-specific transcriptional factors could change the splicing pattern of this segment, then the deletion of $4 \mathrm{bp}$ could disrupt the canonical splicing in some tissues, whereas other remain normal.

\section{TSC1 and TSC2 CNV and genetic counseling implications}

Seven segmental deletions and one segmental duplication have been identified in this study as pathogenic alterations (8/102; 7.84\%). One deleted segment extended from TSC1 intron 8 to at least the 
downstream gene (SPACA9), having a length of more than $23 \mathrm{~Kb}$. Although its breakpoints have not been sequenced, the segment overlaps part of a deletion reported by van den Ouweland, Elfferich et al. (2011) with a breakpoint mapping to nearly 1,400 bases downstream intron 8 and exon 9 junction, and extending downstream for at least $119,000 \mathrm{~kb}$.

The deletion of full-length TSC2 (TSC2:c.(?_-30)_(*102_?)del) extended downstream to PKD1 exons 46 but not exon 45, and upstream to exon 6 of NTHL1. Homozygosis pathogenic DNA variants in this gene relate to an autosomal recessive cancer predisposition syndrome, often with progression to colorectal cancer (Weren, Ligtenberg et al. 2015). In this report, the most frequent NTHL1 mutation had a frequency of 1:250, constituting a high risk for the patient that already has one allele deleted. Therefore, sequencing the most frequent NTHL1 pathogenic DNA variant should be an alternative for homozygosity verification in this patient.

Four intragenic deletions were identified on TSC2. In only one case the two breakpoints could be sequenced. Of those that break point were not sequenced, the length of flanking introns prevented the sequencing of break point, even in the deletion of exon 9. However, the TSC2 internal deletion which had both breakpoints sequenced mapped to introns 10 and 16. Each intron breakpoint had SINE nearby (Figure II-17). Alu high copy number in the human genome and similarity with members from this SINE family allow for unequal recombination (Gu, Yuan et al. 2015). Alu repeats from TSC2 introns 10 and 16 are 66\% identical. The relatively long distance between them (deletion size of $6.2 \mathrm{~Kb}$ ) rules out the possibility of DNA replicationmediated deletion, and reinforces unequal recombination as a likely pathway to have generated the deletion (Abeysinghe, Chuzhanova et al. 2003, Abo-Dalo, Kutsche et al. 2010, Gu, Yuan et al. 2015). Moreover, deletion breakpoints from both cancer and inherited disease are commonly associated to AT-rich sequences (Abeysinghe, Chuzhanova et al. 2003), as found in Alu sequences such as those from TSC2 introns 10 and 16. 
A

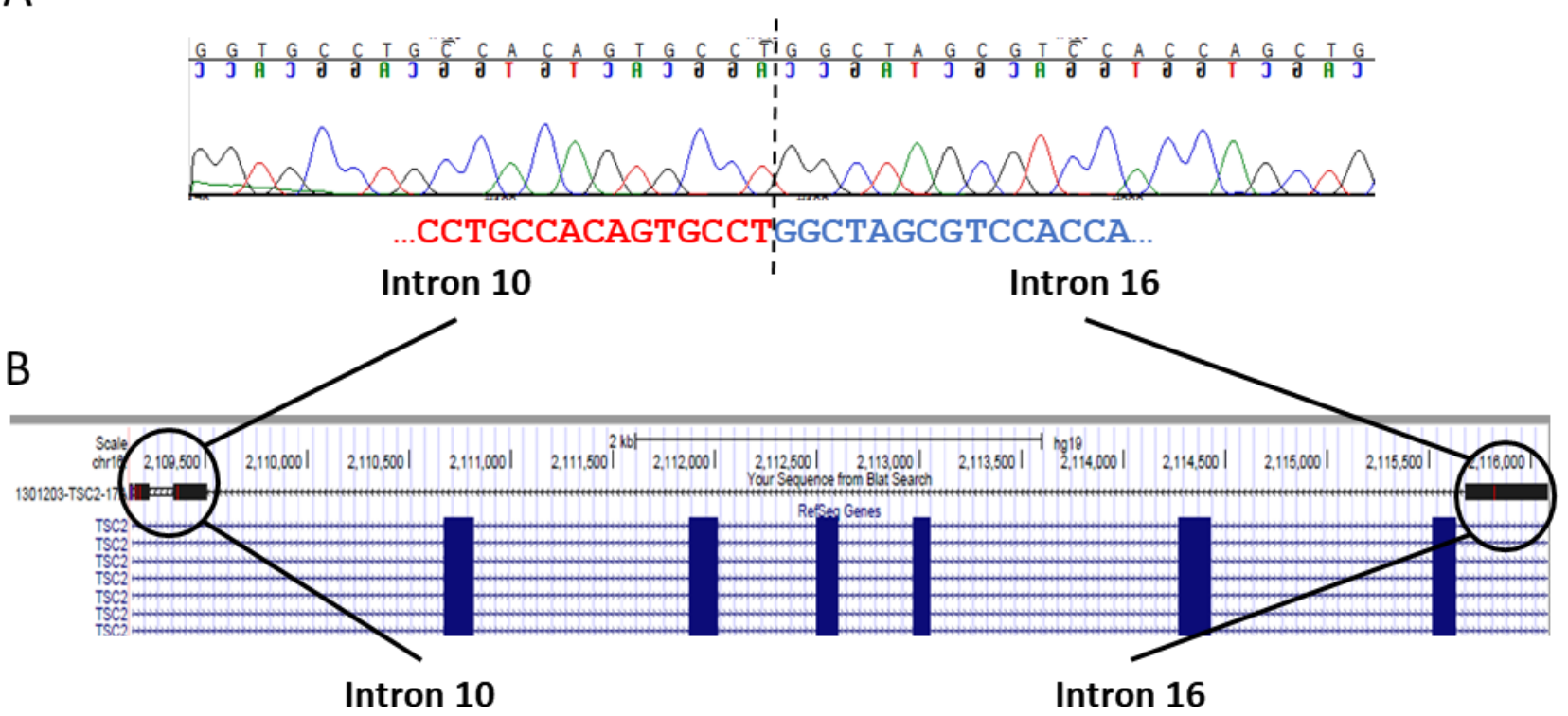

...CCTGCCACAGTGCCTNNNNNN/ / NNNNNNNGGCTAGCGTCCACCA..

C

$$
\text { LINE/L1 class } \Rightarrow \text { SINE/Alu class }
$$

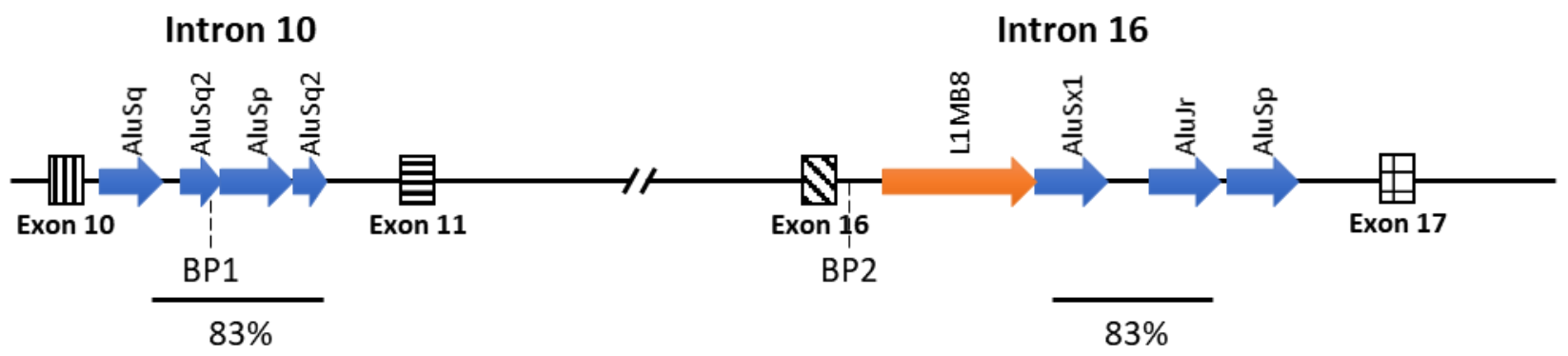

Figure II-17: Breakpoint mapping of the TSC2 intragenic deletion c.(975+_976-)_(1716+_1717-)del . (A) Sanger sequencing of the DNA from the patient with the intragenic TSC2 deletion c.(975+_976-)_(1716+_1717-)del. (B) UCSC genome browser mapping of the breakpoints in TSC2 introns 10 and 16. (C) Diagram illustrating the positions of SINE/Alu and LINE/L1 repeat sequences in TSC2 introns 10 and 16, respectively within and adjacent to each breakpoint. The regions of $66 \%$ similarity between introns are indicated. (D) Steps representing the predicted intra-chromosomal crossing-over leading to deletion. 1: normal chromosomal pairing; 2: intrachromosomal pairing. Red cross represents the crossing over. 3: Daughter cells with normal and TSC2 intragenic deleted chromosome (continue on next page). 
D

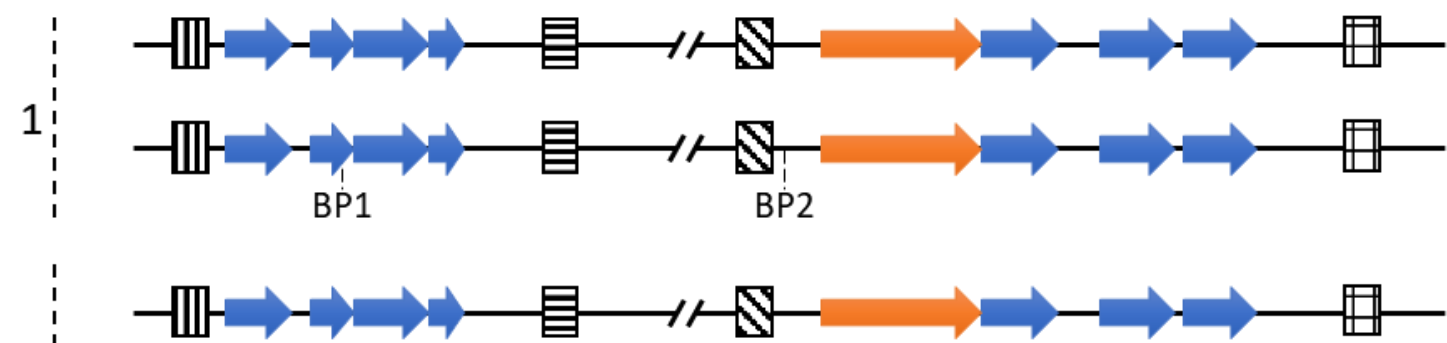

\section{BP1}
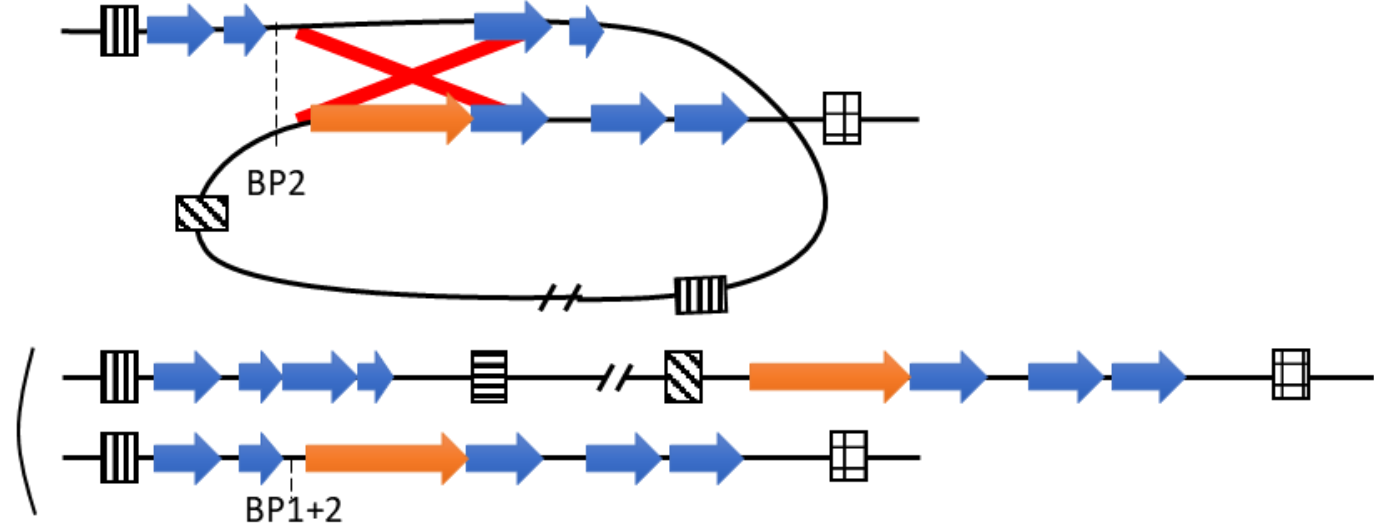

3

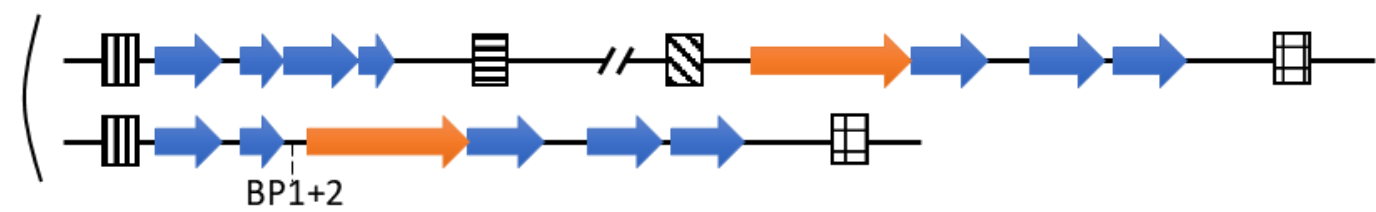

\section{NMI patients}

An NMI patient presented a mosaic TSC1 exon 1 deletion. The mosaic deletion was confirmed by QPCR, and deletion of upstream gene GFI1B has been ruled out. MLPA false-positive deletion of only one exon can be due to SNV impairing probe hybridization (van Dijk, Rombout et al. 2005, Kim, Cho et al. 2016). However, no DNA variant has been detected by Sanger sequencing of the patient TSC1 exon 1. Due to the long extent of TSC1 intron 1 (9,447 bp) and intergenic segment upstream (nearly $34 \mathrm{~Kb}$ ), we could not map the breakpoints of TSC1 exon 1 deletion from the patient by a PCR-based approach with different primer pairs (data not shown). A study by van den Ouweland, Elfferich et al. (2011) identified five out of 327 TSC patients with deletions affecting TSC1 exon 1, preserving exon 2. Upstream breakpoints from four deletions mapped differently between TSC1 and GFI1B genes. Likewise, downstream breakpoints from the four cases mapped on TSC1 intron 1 between 300 and more than $4 \mathrm{~Kb}$ away the exon-intron junction. In one case of non-mosaic TSC1 exon 1 deletion, only the wild-type allele was expressed, suggesting the deletion inactivated transcription of the gene (van den Ouweland, Elfferich et al. 2011). As we could not evaluate the TSC1 mRNA levels from our patient, the patient classified as NMI and the mosaic TSC1 exon 1 deletion of uncertain significance. If this 
is definitely not the TSC-causing mutation, it remains a possibility that the patient may have a pathogenic alteration in mosaicism that could not have been detected by sequencing due to limited sensitivity.

The second NMI patient had the c.1361+54_1361+57del variant in TSC2 gene intron 13, which did not affect the splicing of the minigene primary transcript in HEK293 cell line (Figure II-10). Since the patient mother has facial angiofibromas, we ruled out the possibility that the disease in the affected child is due to a novel mutation in mosaicism. As the patient had no segmental alteration on TSC1 or TSC2 detected by Sanger sequencing, NGS-Panel and MLPA, it is more likely that a DNA variant belongs to deep intronic sequence do not cover by our primer designed or to sequence that potentially altering TSC1/2 gene expression regulation causes the disease.

NMI patients tend to correspond to less severe TSC cases (Camposano, Greenberg et al. 2009). This class of patient should be submitted to whole gene-targeted NGS to access the deep intronic regions and possible mosaics (Nellist, Brouwer et al. 2015, Tyburczy, Dies et al. 2015). Currently, six VUS are under functional investigation: c.1119G >A (p.=), c.1443G>T (p.Glu481Asp), c.1663G>C (p.Ala555Pro), c.4493G>T (p.Ser1498Ile), c.5051C>T (p.Ser1684Phe) and c.5228G>C (p.Arg1743Pro).

Of note, one splicing mutation is a deep intronic variant (c.2838-122G>A) previously reported as pathogenic by a functional assay (Nellist, Brouwer et al. 2015) and appear in two unrelated patients. As demonstrated by recent reports (Nellist, Brouwer et al. 2015, Tyburczy, Dies et al. 2015), deep intronic DNA variants affecting splicing along with somatic mosaicism explains part of the NMI cases. Identification of deep intronic variants was possible because in our study many primers designed for Sanger sequencing annealed on intronic sequences at least 200 bp away the exon/intron junctions.

Somatic mosaicism has been described in many individuals with TSC. The likely overestimate of the percent of mosaicism in some studies because are caused by the sensitivity of the pathogenic variant screening methods and the difficulty of detecting low-level mosaicism. It has been estimated that levels of mosaicism greater than $20 \%$ in lymphocyte DNA can be detected with confidence by Sanger sequencing and nextgeneration sequencing methods (Qin, Kozlowski et al. 2010). Using targeted deep next-generation sequencing of TSC1 and TSC2, somatic mosaic pathogenic variants at $9.5 \%$ in an affected individual who did not have a pathogenic variant identified by exon sequence analysis or gene-targeted deletion/duplication analysis was reported (Nellist, Brouwer et al. 2015). However, it has not yet been determined whether levels of somatic mosaicism below $9.5 \%$ can be detected by deep next-generation sequencing. Although detection of somatic mosaicism between $1 \%$ and $9.5 \%$ has been reported (Tyburczy, Dies et al. 2015). 


\section{Conclusions}

We analyzed DNA from 117 patients with definite clinical diagnosis of TSC. We report a mutation detection rate of $87,2 \%(102 / 117,41$ novel pathogenic DNA variants as well as 19 novel SNV. Nonsense and frameshifting pathogenic variants corresponded altogether to $94.4 \%$ and $50 \%$, respectively, of TSC1 and TSC2 pathogenic DNA alterations. Missense, in-frame deletion and splicing DNA variants have been detected only in the TSC2 gene. Segmental gene deletions and duplications comprehended $7.8 \%$ of all pathogenic DNA alterations. Primers designed more deeply in introns could identify four deep intronic DNA variants. These variants may explain part of NMI patients but require experimental assessment for pathogenicity confirmation. Our rate of pathogenic alteration detection could have been lower without a deeper analysis of intronic DNA sequence.

Among 20 rare missense variants and five rare in-frame deletion variants, at least two in silico bioinformatics tools predicted damaging effects. The mTOR-based functional assay disclosed non-overlapping results for in silico prediction of two missense variants but confirmed pathogenicity of five variants. 


\section{References}

Abeysinghe, S. S., N. Chuzhanova, M. Krawczak, E. V. Ball and D. N. Cooper (2003). "Translocation and gross deletion breakpoints in human inherited disease and cancer I: Nucleotide composition and recombination-associated motifs." Hum Mutat 22(3): 229-244.

Abo-Dalo, B., K. Kutsche, V. Mautner and L. Kluwe (2010). "Large intragenic deletions of the NF2 gene: breakpoints and associated phenotypes." Genes Chromosomes Cancer 49(2): 171-175.

Adzhubei, I. A., S. Schmidt, L. Peshkin, V. E. Ramensky, A. Gerasimova, P. Bork, A. S. Kondrashov and S. R. Sunyaev (2010). "A method and server for predicting damaging missense mutations." Nat Methods 7(4): 248-249.

Ambar, G. and S. Chiavegatto (2009). "Anabolic-androgenic steroid treatment induces behavioral disinhibition and downregulation of serotonin receptor messenger RNA in the prefrontal cortex and amygdala of male mice." Genes Brain Behav 8(2): 161-173.

Au, K. S., J. A. Rodriguez, J. L. Finch, K. A. Volcik, E. S. Roach, M. R. Delgado, E. Rodriguez, Jr. and H. Northrup (1998). "Germ-line mutational analysis of the TSC2 gene in 90 tuberous-sclerosis patients." Am J Hum Genet 62(2): 286-294.

Au, K. S., A. T. Williams, E. S. Roach, L. Batchelor, S. P. Sparagana, M. R. Delgado, J. W. Wheless, J. E. Baumgartner, B. B. Roa, C. M. Wilson, T. K. Smith-Knuppel, M. Y. Cheung, V. H. Whittemore, T. M. King and H. Northrup (2007). "Genotype/phenotype correlation in 325 individuals referred for a diagnosis of tuberous sclerosis complex in the United States." Genet Med 9(2): 88-100.

Beauchamp, R. L., A. Banwell, P. McNamara, M. Jacobsen, E. Higgins, H. Northrup, P. Short, K. Sims, L. Ozelius and V. Ramesh (1998) "Exon scanning of the entire TSC2 gene for germline mutations in 40 unrelated patients with tuberous sclerosis." Hum Mutat 12(6): 408416.

Camposano, S. E., E. Greenberg, D. J. Kwiatkowski and E. A. Thiele (2009). "Distinct clinical characteristics of tuberous sclerosis complex patients with no mutation identified." Ann Hum Genet 73(2): 141-146.

Choi, Y. and A. P. Chan (2015). "PROVEAN web server: a tool to predict the functional effect of amino acid substitutions and indels." Bioinformatics 31(16): 2745-2747.

Chong-Kopera, H., K. Inoki, Y. Li, T. Zhu, F. R. Garcia-Gonzalo, J. L. Rosa and K. L. Guan (2006). "TSC1 stabilizes TSC2 by inhibiting the interaction between TSC2 and the HERC1 ubiquitin ligase." J Biol Chem 281(13): 8313-8316.

Consortium, T. E. C. T. S. (1993). "Identification and characterization of the tuberous sclerosis gene on chromosome 16." Cell 75(7): $1305-1315$.

Dabora, S. L., D. N. Franz, S. Ashwal, A. Sagalowsky, F. J. DiMario, Jr., D. Miles, D. Cutler, D. Krueger, R. N. Uppot, R. Rabenou, S Camposano, J. Paolini, F. Fennessy, N. Lee, C. Woodrum, J. Manola, J. Garber and E. A. Thiele (2011). "Multicenter phase 2 trial of sirolimus for tuberous sclerosis: kidney angiomyolipomas and other tumors regress and VEGF- D levels decrease." PLoS One 6(9): e23379.

Dabora, S. L., S. Jozwiak, D. N. Franz, P. S. Roberts, A. Nieto, J. Chung, Y. S. Choy, M. P. Reeve, E. Thiele, J. C. Egelhoff, J. Kasprzyk-Obara, D. Domanska-Pakiela and D. J. Kwiatkowski (2001). "Mutational analysis in a cohort of 224 tuberous sclerosis patients indicates increased severity of TSC2, compared with TSC1, disease in multiple organs." Am J Hum Genet 68(1): 64-80.

Desmet, F. O., D. Hamroun, M. Lalande, G. Collod-Beroud, M. Claustres and C. Beroud (2009). "Human Splicing Finder: an online bioinformatics tool to predict splicing signals." Nucleic Acids Res 37(9): e67.

Dufner-Almeida, L. (2014). Estudo mutacional em pacientes com o complexo da esclerose tuberosa. Dissertação de Mesteado Dissertação de Mestrado, Universidade de São Paulo.

Fagerberg, L., B. M. Hallstrom, P. Oksvold, C. Kampf, D. Djureinovic, J. Odeberg, M. Habuka, S. Tahmasebpoor, A. Danielsson, K. Edlund A. Asplund, E. Sjostedt, E. Lundberg, C. A. Szigyarto, M. Skogs, J. O. Takanen, H. Berling, H. Tegel, J. Mulder, P. Nilsson, J. M. Schwenk, C. 
Lindskog, F. Danielsson, A. Mardinoglu, A. Sivertsson, K. von Feilitzen, M. Forsberg, M. Zwahlen, I. Olsson, S. Navani, M. Huss, J. Nielsen, F. Ponten and M. Uhlen (2014). "Analysis of the human tissue-specific expression by genome-wide integration of transcriptomics and antibody-based proteomics." Mol Cell Proteomics 13(2): 397-406.

Freeman, P. J., R. K. Hart, L. J. Gretton, A. J. Brookes and R. Dalgleish (2018). "VariantValidator: Accurate validation, mapping, and formatting of sequence variation descriptions." Hum Mutat 39(1): 61-68.

Frey, U. H., H. S. Bachmann, J. Peters and W. Siffert (2008). "PCR-amplification of GC-rich regions: 'slowdown PCR'." Nat Protoc 3(8): 1312-1317.

Garami, A., F. J. T. Zwartkruis, T. Nobukuni, M. Joaquin, M. Roccio, H. Stocker, S. C. Kozma, E. Hafen, J. L. Bos and G. Thomas (2003). "Insulin Activation of Rheb, a Mediator of mTOR/S6K/4E-BP Signaling, Is Inhibited by TSC1 and 2." Molecular Cell 11(6): 1457-1466.

Gerstein, M. B., C. Bruce, J. S. Rozowsky, D. Zheng, J. Du, J. O. Korbel, O. Emanuelsson, Z. D. Zhang, S. Weissman and M. Snyder (2007) "What is a gene, post-ENCODE? History and updated definition." Genome Res 17(6): 669-681.

Goncharova, E. A., D. A. Goncharov, A. Eszterhas, D. S. Hunter, M. K. Glassberg, R. S. Yeung, C. L. Walker, D. Noonan, D. J. Kwiatkowski, M. M. Chou, R. A. Panettieri, Jr. and V. P. Krymskaya (2002). "Tuberin regulates p70 S6 kinase activation and ribosomal protein S6 phosphorylation. A role for the TSC2 tumor suppressor gene in pulmonary lymphangioleiomyomatosis (LAM)." J Biol Chem 277(34): 30958-30967.

Gu, S., B. Yuan, I. M. Campbell, C. R. Beck, C. M. Carvalho, S. C. Nagamani, A. Erez, A. Patel, C. A. Bacino, C. A. Shaw, P. Stankiewicz, S. W. Cheung, W. Bi and J. R. Lupski (2015). "Alu-mediated diverse and complex pathogenic copy-number variants within human chromosome 17 at p13.3." Hum Mol Genet 24(14): 4061-4077.

Hoogeveen-Westerveld, M., R. Ekong, S. Povey, I. Karbassi, S. D. Batish, J. T. den Dunnen, A. van Eeghen, E. Thiele, K. Mayer, K. Dies, L. Wen, C. Thompson, S. P. Sparagana, P. Davies, C. Aalfs, A. van den Ouweland, D. Halley and M. Nellist (2012). "Functional assessment of TSC1 missense variants identified in individuals with tuberous sclerosis complex." Hum Mutat 33(3): 476-479.

Hoogeveen-Westerveld, M., R. Ekong, S. Povey, K. Mayer, N. Lannoy, F. Elmslie, M. Bebin, K. Dies, C. Thompson, S. P. Sparagana, P. Davies, A. M. van Eeghen, E. A. Thiele, A. van den Ouweland, D. Halley and M. Nellist (2013). "Functional assessment of TSC2 variants identified in individuals with tuberous sclerosis complex." Hum Mutat 34(1): 167-175.

Hoogeveen-Westerveld, M., L. van Unen, A. van den Ouweland, D. Halley, A. Hoogeveen and M. Nellist (2012). "The TSC1-TSC2 complex consists of multiple TSC1 and TSC2 subunits." BMC Biochem 13: 18.

Hoogeveen-Westerveld, M., M. Wentink, D. van den Heuvel, M. Mozaffari, R. Ekong, S. Povey, J. T. den Dunnen, K. Metcalfe, S. Vallee, S. Krueger, J. Bergoffen, V. Shashi, F. Elmslie, D. Kwiatkowski, J. Sampson, C. Vidales, J. Dzarir, J. Garcia-Planells, K. Dies, A. Maat-Kievit, A. van den Ouweland, D. Halley and M. Nellist (2011). "Functional assessment of variants in the TSC1 and TSC2 genes identified in individuals with Tuberous Sclerosis Complex." Hum Mutat 32(4): 424-435.

Hornbeck, P. V., B. Zhang, B. Murray, J. M. Kornhauser, V. Latham and E. Skrzypek (2015). "PhosphoSitePlus, 2014: mutations, PTMs and recalibrations." Nucleic Acids Res 43(Database issue): D512-520.

Hung, C. C., Y. N. Su, S. C. Chien, H. H. Liou, C. C. Chen, P. C. Chen, C. J. Hsieh, C. P. Chen, W. T. Lee, W. L. Lin and C. N. Lee (2006). "Molecular and clinical analyses of 84 patients with tuberous sclerosis complex." BMC Med Genet 7: 72.

Inoki, K., Y. Li, T. Xu and K. L. Guan (2003). "Rheb GTPase is a direct target of TSC2 GAP activity and regulates mTOR signaling." Genes Dev 17(15): 1829-1834.

Jones, A. C., M. M. Shyamsundar, M. W. Thomas, J. Maynard, S. Idziaszczyk, S. Tomkins, J. R. Sampson and J. P. Cheadle (1999). "Comprehensive mutation analysis of TSC1 and TSC2-and phenotypic correlations in 150 families with tuberous sclerosis." Am J Hum Genet 64(5): 1305-1315. 
Kim, M. J., S. I. Cho, J. H. Chae, B. C. Lim, J. S. Lee, S. J. Lee, S. H. Seo, H. Park, A. Cho, S. Y. Kim, J. Y. Kim, S. S. Park and M. W. Seong (2016). "Pitfalls of Multiple Ligation-Dependent Probe Amplifications in Detecting DMD Exon Deletions or Duplications." J Mol Diagn 18(2): 253259.

Knudson, A. G., Jr. (1971). "Mutation and cancer: statistical study of retinoblastoma." Proc Natl Acad Sci U S A 68(4): 820-823.

Kozlowski, P., P. Roberts, S. Dabora, D. Franz, J. Bissler, H. Northrup, K. S. Au, R. Lazarus, D. Domanska-Pakiela, K. Kotulska, S. Jozwiak and D. J. Kwiatkowski (2007). "Identification of 54 large deletions/duplications in TSC1 and TSC2 using MLPA, and genotype-phenotype correlations." Hum Genet 121(3-4): 389-400.

Kumar, P., S. Henikoff and P. C. Ng (2009). "Predicting the effects of coding non-synonymous variants on protein function using the SIFT algorithm." Nat Protoc 4(7): 1073-1081.

Kwiatkowski, D. J., M. R. Palmer, S. Jozwiak, J. Bissler, D. Franz, S. Segal, D. Chen and J. R. Sampson (2015). "Response to everolimus is seen in TSC-associated SEGAs and angiomyolipomas independent of mutation type and site in TSC1 and TSC2." Eur J Hum Genet 23(12): 1665-1672.

Li, H. and R. Durbin (2009). "Fast and accurate short read alignment with Burrows-Wheeler Transform. Bioinformatics." Bioinformatics 25: 7 .

Li, W., L. H. Zhou, B. D. Gao, L. Y. Li, C. G. Zhong, F. Gong, H. M. Xiao, T. Song and G. X. Lu (2011). "[Mutation screening and prenatal diagnosis of tuberous sclerosis complex]." Zhonghua Yi Xue Yi Chuan Xue Za Zhi 28(4): 361-366.

Martignoni, G., F. Bonetti, M. Pea, R. Tardanico, M. Brunelli and J. N. Eble (2002). "Renal disease in adults with TSC2/PKD1 contiguous gene syndrome." Am J Surg Pathol 26(2): 198-205.

McKenna, A., M. Hanna, E. Banks, A. Sivachenko, K. Cibulskis, A. Kernytsky, K. Garimella, D. Altshuler, S. Gabriel, M. Daly and M. A. DePristo (2010). "The Genome Analysis Toolkit: a MapReduce framework for analyzing next-generation DNA sequencing data." Genome Res 20(9): 1297-1303.

Moavero, R., M. Pinci, R. Bombardieri and P. Curatolo (2011). "The management of subependymal giant cell tumors in tuberous sclerosis: a clinician's perspective." Childs Nerv Syst 27(8): 1203-1210.

Nellist, M., R. W. Brouwer, C. E. Kockx, M. van Veghel-Plandsoen, C. Withagen-Hermans, L. Prins-Bakker, M. Hoogeveen-Westerveld, A Mrsic, M. M. van den Berg, A. E. Koopmans, M. C. de Wit, F. E. Jansen, A. J. Maat-Kievit, A. van den Ouweland, D. Halley, A. de Klein and I. W. F. van (2015). "Targeted Next Generation Sequencing reveals previously unidentified TSC1 and TSC2 mutations." BMC Med Genet 16: 10.

Nellist, M., O. Sancak, M. A. Goedbloed, C. Rohe, D. van Netten, K. Mayer, A. Tucker-Williams, A. M. van den Ouweland and D. J. Halley (2005). "Distinct effects of single amino-acid changes to tuberin on the function of the tuberin-hamartin complex." Eur J Hum Genet 13(1): 59-68

Ng, P. C. and S. Henikoff (2006). "Predicting the effects of amino acid substitutions on protein function." Annu Rev Genomics Hum Genet 7: $61-80$.

Niida, Y., N. Lawrence-Smith, A. Banwell, E. Hammer, J. Lewis, R. L. Beauchamp, K. Sims, V. Ramesh and L. Ozelius (1999). "Analysis of bothTSC1 andTSC2 for germline mutations in 126 unrelated patients with tuberous sclerosis." Human Mutation 14(5): 412-422.

Niida, Y., A. O. Stemmer-Rachamimov, M. Logrip, D. Tapon, R. Perez, D. J. Kwiatkowski, K. Sims, M. MacCollin, D. N. Louis and V. Ramesh (2001). "Survey of somatic mutations in tuberous sclerosis complex (TSC) hamartomas suggests different genetic mechanisms for pathogenesis of TSC lesions." Am J Hum Genet 69(3): 493-503. 
Northrup, H., D. A. Krueger and G. International Tuberous Sclerosis Complex Consensus (2013). "Tuberous sclerosis complex diagnostic criteria update: recommendations of the 2012 linternational Tuberous Sclerosis Complex Consensus Conference." Pediatr Neurol 49(4): 243-254.

O'Callaghan, F. J., A. W. Shiell, J. P. Osborne and C. N. Martyn (1998). "Prevalence of tuberous sclerosis estimated by capture-recapture analysis." Lancet 351(9114): 1490.

O'Callaghan, T. J., J. A. Edwards, M. Tobin and B. K. Mookerjee (1975). "Tuberous sclerosis with striking renal involvement in a family." Archives of Internal Medicine 135(8): 1082-1087.

Osborne, J. P., A. Fryer and D. Webb (1991). "Epidemiology of tuberous sclerosis." Ann N Y Acad Sci 615: 125-127.

Padma Priya, T. and A. B. Dalal (2012). "Tuberous sclerosis: diagnosis and prenatal diagnosis by MLPA." Indian J Pediatr 79(10): 13661369.

Peron, A., K. S. Au and H. Northrup (2018). "Genetics, genomics, and genotype-phenotype correlations of TSC: Insights for clinical practice." Am J Med Genet C Semin Med Genet 178(3): 281-290.

Piva, F., M. Giulietti, A. B. Burini and G. Principato (2012). "SpliceAid 2: a database of human splicing factors expression data and RNA target motifs." Hum Mutat 33(1): 81-85.

Pujol, P., P. Vande Perre, L. Faivre, D. Sanlaville, C. Corsini, B. Baertschi, M. Anahory, D. Vaur, S. Olschwang, N. Soufir, N. Bastide, S. Amar, M. Vintraud, O. Ingster, S. Richard, P. Le Coz, J. P. Spano, O. Caron, P. Hammel, E. Luporsi, A. Toledano, X. Rebillard, A. Cambon-Thomsen, O. Putois, J. M. Rey, C. Herve, C. Zorn, K. Baudry, V. Galibert, J. Gligorov, D. Azria, B. Bressac-de Paillerets, N. Burnichon, M. Spielmann, D. Zarca, I. Coupier, O. Cussenot, A. P. Gimenez-Roqueplo, S. Giraud, A. S. Lapointe, P. Niccoli, I. Raingeard, M. Le Bidan, T. Frebourg, A. Rafii and D. Genevieve (2018). "Guidelines for reporting secondary findings of genome sequencing in cancer genes: the SFMPP recommendations." Eur J Hum Genet.

Qin, W., J. A. Chan, H. V. Vinters, G. W. Mathern, D. N. Franz, B. E. Taillon, P. Bouffard and D. J. Kwiatkowski (2010). "Analysis of TSC cortical tubers by deep sequencing of TSC1, TSC2 and KRAS demonstrates that small second-hit mutations in these genes are rare events." Brain Pathol 20(6): 1096-1105.

Qin, W., P. Kozlowski, B. E. Taillon, P. Bouffard, A. J. Holmes, P. Janne, S. Camposano, E. Thiele, D. Franz and D. J. Kwiatkowski (2010). "Ultra deep sequencing detects a low rate of mosaic mutations in tuberous sclerosis complex." Hum Genet 127(5): 573-582.

Rakowski, S. K., E. B. Winterkorn, E. Paul, D. J. Steele, E. F. Halpern and E. A. Thiele (2006). "Renal manifestations of tuberous sclerosis complex: Incidence, prognosis, and predictive factors." Kidney Int 70(10): 1777-1782.

Rendtorff, N. D., B. Bjerregaard, M. Frodin, S. Kjaergaard, H. Hove, F. Skovby, K. Brondum-Nielsen, M. Schwartz and G. Danish Tuberous Sclerosis (2005). "Analysis of 65 tuberous sclerosis complex (TSC) patients by TSC2 DGGE, TSC1/TSC2 MLPA, and TSC1 long-range PCR sequencing, and report of 28 novel mutations." Hum Mutat 26(4): 374-383.

Richards, S., N. Aziz, S. Bale, D. Bick, S. Das, J. Gastier-Foster, W. W. Grody, M. Hegde, E. Lyon, E. Spector, K. Voelkerding, H. L. Rehm and A. L. Q. A. Committee (2015). "Standards and guidelines for the interpretation of sequence variants: a joint consensus recommendation of the American College of Medical Genetics and Genomics and the Association for Molecular Pathology." Genet Med 17(5): $405-424$.

Riemenschneider, M. J., R. A. Betensky, S. M. Pasedag and D. N. Louis (2006). "AKT activation in human glioblastomas enhances proliferation via TSC2 and S6 kinase signaling." Cancer Res 66(11): 5618-5623.

Roach, E. S., M. R. Gomez and H. Northrup (1998). "Tuberous sclerosis complex consensus conference: revised clinical diagnostic criteria." J Child Neurol 13(12): 624-628. 
Rosset, C., F. Vairo, I. C. Bandeira, R. L. Correia, F. V. de Goes, R. T. B. da Silva, L. S. M. Bueno, M. C. S. de Miranda Gomes, H. C. R. Galvao, J. Neri, M. I. Achatz, C. B. O. Netto and P. Ashton-Prolla (2017). "Molecular analysis of TSC1 and TSC2 genes and phenotypic correlations in Brazilian families with tuberous sclerosis." PLoS One 12(10): e0185713.

Rozen, S. and H. J. Skaletsky (2000). "Primer3 on the WWW for general users and for biologist programmers. In: Krawetz S, Misener S (eds) Bioinformatics Methods and Protocols: Methods in Molecular Biology." Humana Press: 2.

Sampson, J. R., S. J. Scahill, J. B. Stephenson, L. Mann and J. M. Connor (1989). "Genetic aspects of tuberous sclerosis in the west of Scotland." J Med Genet 26(1): 28-31.

Sancak, O., M. Nellist, M. Goedbloed, P. Elfferich, C. Wouters, A. Maat-Kievit, B. Zonnenberg, S. Verhoef, D. Halley and A. van den Ouweland (2005). "Mutational analysis of the TSC1 and TSC2 genes in a diagnostic setting: genotype--phenotype correlations and comparison of diagnostic DNA techniques in Tuberous Sclerosis Complex." Eur J Hum Genet 13(6): 731-741.

Schwarz, J. M., C. Rodelsperger, M. Schuelke and D. Seelow (2010). "MutationTaster evaluates disease-causing potential of sequence alterations." Nat Methods 7(8): 575-576.

Stapleton, F. B., D. Johnson, G. W. Kaplan and W. Griswold (1980). "The cystic renal lesion in tuberous sclerosis." The Journal of Pediatrics 97(4): 574-579.

Tee, A. R., D. C. Fingar, B. D. Manning, D. J. Kwiatkowski, L. C. Cantley and J. Blenis (2002). "Tuberous sclerosis complex-1 and -2 gene products function together to inhibit mammalian target of rapamycin (mTOR)-mediated downstream signaling." Proc Natl Acad Sci U S A 99(21): 13571-13576

Tee, A. R., B. D. Manning, P. P. Roux, L. C. Cantley and J. Blenis (2003). "Tuberous Sclerosis Complex Gene Products, Tuberin and Hamartin, Control mTOR Signaling by Acting as a GTPase-Activating Protein Complex toward Rheb." Current Biology 13(15): 1259-1268.

Tyburczy, M. E., K. A. Dies, J. Glass, S. Camposano, Y. Chekaluk, A. R. Thorner, L. Lin, D. Krueger, D. N. Franz, E. A. Thiele, M. Sahin and D. J. Kwiatkowski (2015). "Mosaic and Intronic Mutations in TSC1/TSC2 Explain the Majority of TSC Patients with No Mutation Identified by Conventional Testing." PLoS Genet 11(11): e1005637.

Tyburczy, M. E., J. A. Wang, S. Li, R. Thangapazham, Y. Chekaluk, J. Moss, D. J. Kwiatkowski and T. N. Darling (2013). "Sun exposure causes somatic second-hit mutations and angiofibroma development in tuberous sclerosis complex." Human Molecular Genetics 23(8): 20232029

van den Ouweland, A. M., P. Elfferich, B. A. Zonnenberg, W. F. Arts, T. Kleefstra, M. D. Nellist, J. M. Millan, C. Withagen-Hermans, A. J. Maat-Kievit and D. J. Halley (2011). "Characterisation of TSC1 promoter deletions in tuberous sclerosis complex patients." Eur J Hum Genet 19(2): 157-163

van Dijk, M. C., P. D. Rombout, S. H. Boots-Sprenger, H. Straatman, M. R. Bernsen, D. J. Ruiter and J. W. Jeuken (2005). "Multiplex ligationdependent probe amplification for the detection of chromosomal gains and losses in formalin-fixed tissue." Diagn Mol Pathol 14(1): 9 16

van Slegtenhorst, M., R. de Hoogt, C. Hermans, M. Nellist, B. Janssen, S. Verhoef, D. Lindhout, A. van den Ouweland, D. Halley, J. Young, M. Burley, S. Jeremiah, K. Woodward, J. Nahmias, M. Fox, R. Ekong, J. Osborne, J. Wolfe, S. Povey, R. G. Snell, J. P. Cheadle, A. C. Jones, M. Tachataki, D. Ravine, J. R. Sampson, M. P. Reeve, P. Richardson, F. Wilmer, C. Munro, T. L. Hawkins, T. Sepp, J. B. Ali, S. Ward, A. J. Green, J. R. Yates, J. Kwiatkowska, E. P. Henske, M. P. Short, J. H. Haines, S. Jozwiak and D. J. Kwiatkowski (1997). "Identification of the tuberous sclerosis gene TSC1 on chromosome 9q34." Science 277(5327): 805-808.

van Slegtenhorst, M., M. Nellist, B. Nagelkerken, J. Cheadle, R. Snell, A. van den Ouweland, A. Reuser, J. Sampson, D. Halley and P. van der Sluijs (1998). "Interaction between hamartin and tuberin, the TSC1 and TSC2 gene products." Hum Mol Genet 7(6): 7. 
van Slegtenhorst, M. A., A. Verhoef, A. Tempelaars, L. Bakker, Q. Wang, M. Wessels, R. Bakker, M. Nellist, D. Lindhout, D. Halley and A. van den Ouweland (1999). "Mutational spectrum of the TSC1 gene in a cohort of 225 tuberous sclerosis complex patients: no evidence for genotype-phenotype correlation." J Med Genet 36(4): 5.

Wang, K., M. Li and H. Hakonarson (2010). "ANNOVAR: functional annotation of genetic variants from high-throughput sequencing data." Nucleic Acids Research 38(16): e164-e164.

Weren, R. D., M. J. Ligtenberg, C. M. Kets, R. M. de Voer, E. T. Verwiel, L. Spruijt, W. A. van Zelst-Stams, M. C. Jongmans, C. Gilissen, J. Y. Hehir-Kwa, A. Hoischen, J. Shendure, E. A. Boyle, E. J. Kamping, I. D. Nagtegaal, B. B. Tops, F. M. Nagengast, A. Geurts van Kessel, J. H. van Krieken, R. P. Kuiper and N. Hoogerbrugge (2015). "A germline homozygous mutation in the base-excision repair gene NTHL1 causes adenomatous polyposis and colorectal cancer." Nat Genet 47(6): 668-671.

Wiederholt, W. C., M. R. Gomez and L. T. Kurland (1985). "Incidence and prevalence of tuberous sclerosis in Rochester, Minnesota, 1950 through 1982." Neurology 35(4): 3.

Wildeman, M., E. van Ophuizen, J. T. den Dunnen and P. E. Taschner (2008). "Improving sequence variant descriptions in mutation databases and literature using the Mutalyzer sequence variation nomenclature checker." Hum Mutat 29(1): 6-13.

Wilson, P. J., V. Ramesh, A. Kristiansen, C. Bove, S. Jozwiak, D. J. Kwiatkowski, M. P. Short and J. L. Haines (1996). "Novel Mutations Detected in the TSC2 Hene From Both Sporadic and Familial TSC Patients." Human Molecular Genetics 5(2): 249-256.

Zerbino, D. R., P. Achuthan, W. Akanni, M. R. Amode, D. Barrell, J. Bhai, K. Billis, C. Cummins, A. Gall, C. G. Giron, L. Gil, L. Gordon, L. Haggerty, E. Haskell, T. Hourlier, O. G. Izuogu, S. H. Janacek, T. Juettemann, J. K. To, M. R. Laird, I. Lavidas, Z. Liu, J. E. Loveland, T. Maurel, W. McLaren, B. Moore, J. Mudge, D. N. Murphy, V. Newman, M. Nuhn, D. Ogeh, C. K. Ong, A. Parker, M. Patricio, H. S. Riat, H. Schuilenburg, D. Sheppard, H. Sparrow, K. Taylor, A. Thormann, A. Vullo, B. Walts, A. Zadissa, A. Frankish, S. E. Hunt, M. Kostadima, N. Langridge, F. J. Martin, M. Muffato, E. Perry, M. Ruffier, D. M. Staines, S. J. Trevanion, B. L. Aken, F. Cunningham, A. Yates and P. Flicek (2018). "Ensembl 2018." Nucleic Acids Res 46(D1): D754-D761.

Zhang, H., G. Cicchetti, H. Onda, H. B. Koon, K. Asrican, N. Bajraszewski, F. Vazquez, C. L. Carpenter and D. J. Kwiatkowski (2003). "Loss of Tsc1/Tsc2 activates mTOR and disrupts PI3K-Akt signaling through downregulation of PDGFR." J Clin Invest 112(8): 1223-1233. 


\section{Supplementary Tables}

Supplementary Table II-1: PCR and Sanger sequencing primers for the TSC1 (NG_012386.1).

\begin{tabular}{|c|c|c|}
\hline Targeted segment & Name & Oligonucleotide sequence $\left(5^{\prime}-3^{\prime}\right)$ \\
\hline \multirow{4}{*}{ Promoter + exon 1} & gTSC1_1S & AAATGTTTAGCCCAGGAAGGA \\
\hline & gTSC1_1A & GCCGGAGATAGCGTGTAATAA \\
\hline & gTSC1_2A & CATCTTGGACGTACAGCACCT \\
\hline & gTSC1_2S & CCGTCTATCCTTCCTTTCGAG \\
\hline \multirow{2}{*}{ Exon 2} & gTSC1_3S & TTGGATTTTAACCCGGAACTC \\
\hline & gTSC1_3A & TCAGGCACTGAATACAAGCAA \\
\hline \multirow{2}{*}{ Exon 3} & gTSC1_4A & GGGGTTCACTGCATGATTCT \\
\hline & gTSC1_4S & ССТСТTCATAAAСTCGCCAAAG \\
\hline \multirow{2}{*}{ Exon 4} & gTSC1_5S & CAGAACTGTAATGCTGCACAAA \\
\hline & gTSC1_5A & TTCAAGAATCATGGGTCCTACA \\
\hline \multirow{2}{*}{ Exon 5} & gTSC1_6S & TTTTATCTGCATGACCCTTGC \\
\hline & gTSC1_6A & CCATACTTGCATGGACAAGGT \\
\hline \multirow[b]{2}{*}{ Exon 6} & gTSC1_7S & CAGTAGAGTTGGGGCTCAGTG \\
\hline & gTSC1_7A & GCACCCAAGATATTCCCTCA \\
\hline \multirow{4}{*}{ Exons 7+8 } & gTSC1_8S & CTGAAGAGGAGGGCAGAAGTT \\
\hline & gTSC1_8A & ATTAGTCCTCCGCCTGTGAA \\
\hline & gTSC1_9A & АATTTCCCTGTCTGCCGTTA \\
\hline & gTSC1_29S & CAATCCCTAGGCAGCCACTA \\
\hline \multirow[b]{2}{*}{ Exon 9} & gTSC1_10S & TTTCCATTTTGAGGCTACACC \\
\hline & gTSC1_10A & TTCCAGAGACAAAGTTGCAAAA \\
\hline \multirow{2}{*}{ Exon 10} & gTSC1_28S & АССТАAАACСАСАСАСТАACCС \\
\hline & gTSC1_11A & GGAATGCTAAGTCATCCACGA \\
\hline \multirow{4}{*}{ Exons 11+12 } & gTSC1_12S & GGGAAAATTTCACACTGCTCA \\
\hline & gTSC1_12A & CACACCTTGAGAGCAGCTTGT \\
\hline & gTSC1_13A & CCCAGGGATTTGCAATAAGT \\
\hline & gTSC1_13S & CGGCAGTTTTTCTAATAGTTGG \\
\hline \multirow{4}{*}{ Exons 13+14 } & gTSC1_14S & CATCCCAACAATTTGAGAATCA \\
\hline & gTSC1_14A & GGCATCACTTTACCTGGCATA \\
\hline & gTSC1_15A & TCCCAGAATTTCCTTGTTTCC \\
\hline & gTSC1_15S & CCATGTCCAGCCTTCTCTGT \\
\hline \multirow{4}{*}{ Exon 15} & gTSC1_16S & GGATGCCACTTTTTCTCCTCT \\
\hline & gTSC1_16A & TCCCAATTTAGGTGCACAGAG \\
\hline & gTSC1_17A & GATGACAAAATGATGGGCTGT \\
\hline & gTSC1_17S & CACACCAAAGCAAGCCTTTAC \\
\hline \multirow{4}{*}{ Exons 16+17 } & gTSC1_18S & TTATGCCATTGCAGATTTTGAC \\
\hline & gTSC1_18A & GGAAGGACTGGGAACTCTGAC \\
\hline & gTSC1_19A & ACTTGGCAACACTTGAGATCCT \\
\hline & gTSC1_19S & AAGCTAACAACACATGGGAAGG \\
\hline \multirow{2}{*}{ Exon 18} & gTSC1_20S & GCAAACTGATCCCTGAGAAGA \\
\hline & gTSC1_20A & AGTTGGGGAACCTCTGTCCTA \\
\hline \multirow{2}{*}{ Exon 19} & gTSC1_21S & CAGAATCTTTCTGCAGCATCC \\
\hline & gTSC1_21A & CAGCACCAAAAACATGAACCT \\
\hline \multirow{2}{*}{ Exon 20} & gTSC1_22S & CCATTATGTCAGGGACTGTGAA \\
\hline & gTSC1_22A & TAGCTGGACCACGGAGTAGTG \\
\hline \multirow{4}{*}{ Exons 21+22 } & gTSC1_23S & GCTTGGGGATAGATTTCAAGG \\
\hline & gTSC1_23A & ACACGGAGTGAGCTGAGTGTT \\
\hline & gTSC1_24A & TGCAGCTGTCCTCTGAAAGAT \\
\hline & gTSC1_24S & GTCAAACTCCAGGCAAGGTAA \\
\hline \multirow{4}{*}{ Exon 23} & gTSC1_25S & CATATGGCCACAGGAAGTGTT \\
\hline & gTSC1_28A & CCGTCCCATTTCCACACATG \\
\hline & gTSC1_26A & CAGAAAGGCTACTGGTCATGC \\
\hline & gTSC1_26S & GGGAGACGACTATGGGAGAAG \\
\hline
\end{tabular}


Supplementary Table II-2: PCR and Sanger sequencing primers for the TSC2 (NG_005895.1).

\begin{tabular}{|c|c|c|}
\hline Targeted segment & Name & Oligonucleotide sequence $\left(5^{\prime}-3^{\prime}\right)$ \\
\hline \multirow{4}{*}{ Promoter + exon 1} & gTSC2_1S & CGAGGACAGCAAGTTCACTG \\
\hline & $\mathrm{gTSC} 2 \_1 \mathrm{~A}$ & GTTTGCCGTCTCTCCTCTACC \\
\hline & gTSC2_2A & GAGCTTGCTGGGAGTTGTAGTT \\
\hline & $\mathrm{gTSC} 2 \_2 \mathrm{~S}$ & СTACCTGCTGCAGCCTCTCT \\
\hline \multirow{2}{*}{ Exon 2} & gTSC2_3S & GGTAGAGGAGAGACGGCAAAC \\
\hline & gTSC2_3A & AAGTGTGCCTGAACCAGGTC \\
\hline \multirow{2}{*}{ Exon 3} & gTSC2_4S & CGGCTCGTCAAGTGAATCTT \\
\hline & gTSC2_4A & GTCAGCTGTCAACCATGTTCC \\
\hline \multirow{2}{*}{ Exon 4} & gTSC2_5S & TGAGACTGTCCCATGACTTCC \\
\hline & $\mathrm{gTSC} 2 \_5 \mathrm{~A}$ & AGGGCAAAACAACACCGTAG \\
\hline \multirow{2}{*}{ Exon 5} & gTSC2_43S & CCTGCCCTGTACAATGCTGA \\
\hline & gTSC2_43A & CAAGCCCCAGAGACTCACAG \\
\hline \multirow{2}{*}{ Exon 6} & gTSC2_44S & GATCCTAGTGTCCGTGCGTAG \\
\hline & gTSC2_44A & CGGAGCTGAACTTAGGACCAT \\
\hline \multirow{2}{*}{ Exon 7} & gTSC2_8S & GCTCTCATCTGATGTCTTGGTT \\
\hline & gTSC2_8A & GTCATTGATGCTGTCATCCAC \\
\hline \multirow[b]{2}{*}{ Exon 8} & gTSC2_9S & GTCCCCCATGTAAGTCAGGAT \\
\hline & gTSC2_9A & АTСTССТCССAAAGACAGAGG \\
\hline \multirow{2}{*}{ Exon 9} & gTSC2_10S & CTGTCTCCCATGAATGGTTGT \\
\hline & gTSC2_10A & GGCTAAGTAGTTGGGGAGCAC \\
\hline \multirow{2}{*}{ Exon 10} & gTSC2_45S & GTGTTACTGCTGGCCTCTGT \\
\hline & gTSC2_11A & CAGCTCACTGCACACAGAAAC \\
\hline \multirow{2}{*}{ Exon 11} & gTSC2_12S & GGATTCAGTTGCTGGTCTGTC \\
\hline & gTSC2_12A & ACTAATGCGGTCCTCCAAAGT \\
\hline \multirow[b]{2}{*}{ Exon 12} & gTSC2_46S & СCTCTGGTGCCAAGTCCATG \\
\hline & gTSC2_45A & CCCTAAGCTGAGTGTTCCTGG \\
\hline \multirow{2}{*}{ Exon 13} & gTSC2_14S & CAGTTTCCTCCCACCTGTGT \\
\hline & gTSC2_14A & GGAGCATCTCTCCAGACGAC \\
\hline \multirow{2}{*}{ Exon 14} & gTSC2_15S & GTGCTAGCTTGCTTTCCAGTC \\
\hline & gTSC2_15A & AGACTGGCTGAAACGAACTCA \\
\hline \multirow{2}{*}{ Exon 15} & gTSC2_16S & GCTGCTCCTTGTGAGTTGTG \\
\hline & gTSC2_16A & ACTGTGCAGAAACCAAAATGC \\
\hline \multirow{2}{*}{ Exon 16} & gTSC2_17S & CTCAGAACCATGAGCCTGTGT \\
\hline & gTSC2_17A & AGCGTGTGCTACTGGTATGCT \\
\hline & gTSC2_18S & GTTGATGACTGCCCTGATGAT \\
\hline Exon 17 & gTSC2_18A & TTAGAGCGACAAGCCACAGAT \\
\hline & gTSC2_19S & CAGAGTCCTGTTCAGCCTGTC \\
\hline Exon 18 & gTSC2_19A & GAAGCAAGAGAAGCAGCTGAG \\
\hline & gTSC2_20S & CTACATGTACGCGGGACCTC \\
\hline Exon 19 & gTSC2_20A & GCCTTCTGGACCCTAGAGACA \\
\hline & gTSC2_21S & GTGCCCTACTCCCTGCTCTT \\
\hline Exon 20 & gTSC2_46A & GCTCGCAGTCTTTTGGGGAA \\
\hline & gTSC2_47S & GTGTGTTACTTGGCAGGCAC \\
\hline Exon 21 & gTSC2_22A & GTGGACAGGGAACACTGGAT \\
\hline & gTSC2_23S & GAGTCTGCTCGGGTAGCTCA \\
\hline Exon 22 & gTSC2_23A & ACCTGAGCTCCTGAAGTCACA \\
\hline & gTSC2_24S & TCACGGATCACACAAATGGTA \\
\hline $\operatorname{Exon} 23$ & gTSC2_24A & GAGCCCACCTTAGTGATGAAA \\
\hline & gTSC2_25S & CGCACCTCTACAGGAACTTTG \\
\hline Exon 24 & gTSC2_25A & GAGTGAGCACACCCAGACAGT \\
\hline & gTSC2_26S & TCATCACTAAGGTGGGCTCAG \\
\hline Exon 25 & gTSC2_26A & AACCCCCAATTCCACAAGTAG \\
\hline & gTSC2_27S & ACCCACACACGTTTAATTTGC \\
\hline $\operatorname{Exon} 26$ & gTSC2_27A & GAATACGAAAAGGCCAAAACC \\
\hline & gTSC2_28S & AATGTGGTCCACGTGATTCTC \\
\hline Exons $27+28$ & gTSC2_28A & GACTTAGTCCCCAGGCTGGTA \\
\hline & gTSC2_29S & CGCTCCCTGTCTTCTAGGTCT \\
\hline Exon 29 & gTSC2_29A & CAGAGAAGGGCTCCAGGACT \\
\hline & gTSC2_48S & CTTGAGGCTGGTGGTTTTGC \\
\hline Exon 30 & gTSC2_47A & AGAGGGCCAAGTCTGCAATC \\
\hline & gTSC2_31S & TGAGGGGTGCAAAGAGTAGG \\
\hline Exon 31 & gTSC2_31A & GGAGAACAATGGTGCTGAGG \\
\hline
\end{tabular}




\begin{tabular}{|c|c|c|}
\hline Exon 32 & $\begin{array}{l}\text { gTSC2_32S } \\
\text { gTSC2_32A }\end{array}$ & $\begin{array}{l}\text { GACGTCTATTCACGGGAGGA } \\
\text { CTAAACAGCTGCCACCCATC }\end{array}$ \\
\hline \multirow{2}{*}{ Exon 33} & gTSC2_33S & GTTACGAGGGCTGGTTTCAG \\
\hline & gTSC2_33A & ACACTGCGTGAGCAGAGGTAT \\
\hline \multirow{4}{*}{ Exon 34} & gTSC2_34S & ATACCTCTGCTCACGCAGTGT \\
\hline & gTSC2_34A & AGCTGCAGGAACACGAAACT \\
\hline & gTSC2_35A & СTCTTTAAGGCGTCCСTCTCT \\
\hline & gTSC2_35S & CTGTGGACCTCTCCTTCCAG \\
\hline \multirow{2}{*}{ Exon 35} & gTSC2_36S & AGCCTCCAATGCAGAGAAAGT \\
\hline & gTSC2_36A & GTGTCGTATGATGGGATCTGG \\
\hline \multirow[b]{2}{*}{ Exon 36} & gTSC2_37S & TGTTCCTGCAGCTCTACCATT \\
\hline & gTSC2_37A & TGTCAGCTCACTGACCAACAG \\
\hline \multirow{2}{*}{ Exon 37} & gTSC2_38S & GAGGGAAGAGAGGGAGTCAAG \\
\hline & gTSC2_38A & GGCACCTCCTGATTACTCCA \\
\hline \multirow{2}{*}{ Exon 38} & gTSC2_39S & CTCCCATCCAGTCCTGCTAC \\
\hline & gTSC2_39A & TCTGCACTTGCCAGTTACTCC \\
\hline \multirow{2}{*}{ Exons $39+40$} & gTSC2_49S & CAGAGGGGAAAGTTCAGGGG \\
\hline & gTSC2_40A & GTAGATATCGGTGGGGTTGGA \\
\hline \multirow{2}{*}{ Exon 41} & gTSC2_41S & AAGTCTCCCCAGACATGGAG \\
\hline & gTSC2_41A & CACAAACTCGGTGAAGTCCTC \\
\hline \multirow{2}{*}{ Exon 42} & gTSC2_42S & CCGATATCTACCCCTCCAAGT \\
\hline & gTSC2_48A & CTTCTAGAGCCTCGACACCC \\
\hline
\end{tabular}

Supplementary Table II-3: TSC1 exon 1 slowdown PCR cycle program.

\begin{tabular}{cccc}
\hline Cycles & Time & Temperature & Ramp \\
\hline \multirow{2}{*}{$1 \mathrm{X}$} & $5 \mathrm{~min}$ & $95^{\circ} \mathrm{C}$ & $2,5^{\circ} \mathrm{s}$ \\
\hline \multirow{2}{*}{$53 \mathrm{X}$} & $30 \mathrm{sec}$. & $95^{\circ} \mathrm{C}$ & $2,5^{\circ} \mathrm{s}$ \\
\cline { 2 - 4 } & $30 \mathrm{sec}$. & $70^{\circ} \mathrm{C}(-0,3 \%$ cycle $)$ & $1,5^{\circ} / \mathrm{s}$ \\
\cline { 2 - 4 } & $40 \mathrm{sec}$. & $72^{\circ} \mathrm{C}$ & $2,5^{\circ} / \mathrm{s}$ \\
\hline \multirow{2}{*}{$15 \mathrm{X}$} & $30 \mathrm{sec}$. & $95^{\circ} \mathrm{C}$ & $2,5^{\circ} / \mathrm{s}$ \\
\cline { 2 - 4 } & $30 \mathrm{sec}$. & $58^{\circ} \mathrm{C}$ & $1,5^{\circ} / \mathrm{s}$ \\
\cline { 2 - 4 } & $40 \mathrm{sec}$. & $72^{\circ} \mathrm{C}$ & $2,5^{\circ} / \mathrm{s}$ \\
\hline $1 \mathrm{X}$ & $7 \mathrm{~min}$ & $72^{\circ} \mathrm{C}$ & - \\
\hline $1 \mathrm{X}$ & $\infty$ & $10^{\circ} \mathrm{C}$ & - \\
\hline
\end{tabular}

Supplementary Table II-4: Standard PCR cycle program.

\begin{tabular}{ccc}
\hline Cycles & Time & Temperature \\
\hline $1 \mathrm{X}$ & $5 \mathrm{~min}$ & $95^{\circ} \mathrm{C}$ \\
\hline \multirow{2}{*}{$30 \mathrm{X}$} & $1 \mathrm{~min}$ & $95^{\circ} \mathrm{C}$ \\
\cline { 2 - 3 } & $1 \mathrm{~min}$ & $60^{\circ} \mathrm{C}$ \\
\cline { 2 - 3 } & $1 \mathrm{~min}$ & $72^{\circ} \mathrm{C}$ \\
\hline $1 \mathrm{X}$ & $8 \min$ & $72^{\circ} \mathrm{C}$ \\
\hline $1 \mathrm{X}$ & $\infty$ & $4{ }^{\circ} \mathrm{C}$ \\
\hline
\end{tabular}


Supplementary Table II-5: Q-PCR primers for TSC1 (NM_000368.4), TSC2 (NM_000548.3), GAPDH (NM_002046), GFI1B (NM_004188.6), SPACA9 (NM_001316898.1), NTHL1 (NM_002528) and PKD1 (NM_001009944).

\begin{tabular}{|c|c|c|c|c|c|}
\hline Segment & Name & Oligonucleotide sequence $\left(5^{\prime}-3^{\prime}\right)$ & $\begin{array}{l}\text { Product } \\
\text { size (bp) }\end{array}$ & $\begin{array}{c}\text { DNA } \\
\text { concentration } \\
(\mathrm{ng} / \mu \mathrm{L})\end{array}$ & Efficiency \\
\hline \multicolumn{6}{|c|}{ TSC1 } \\
\hline Exon 1 & $\begin{array}{l}\text { qPCRgTSC1_1S } \\
\text { qPCRgTSC1_1A }\end{array}$ & $\begin{array}{l}\text { AGGGACTGTGAGGTAAACAGC } \\
\text { AGGAAGCCCCCATAAAAAGGAG }\end{array}$ & 85 & 3.125 & 2.0111 \\
\hline Exon 17 & $\begin{array}{l}\text { qPCRgTSC1_4S } \\
\text { qPCRgTSC1_4A }\end{array}$ & $\begin{array}{l}\text { CAGATGAGATCCGCACCCTC } \\
\text { AGCTGCTGCTTTGATCACCT }\end{array}$ & 131 & 3.125 & 2.0324 \\
\hline \multicolumn{6}{|c|}{ TSC2 } \\
\hline Exon 9 & $\begin{array}{l}\text { qPCRgTSC2_7S } \\
\text { qPCRgTSC2_7A }\end{array}$ & $\begin{array}{l}\text { CCACAGCGCCATCTACAACA } \\
\text { AAGTCATGGGCCTCGCTCTA }\end{array}$ & 195 & 3.125 & 1.9019 \\
\hline Exon 12 & $\begin{array}{l}\text { qPCRgTSC2_2S } \\
\text { qPCRgTSC2_2A }\end{array}$ & $\begin{array}{l}\text { TCCATGACCTGTTGACCACG } \\
\text { CGCACATCTCTCCACCAGTT }\end{array}$ & 95 & 3.125 & 2.0527 \\
\hline Exon 19 & $\begin{array}{l}\text { qPCRgTSC2_4S } \\
\text { gPCRgTSC2_4A }\end{array}$ & $\begin{array}{l}\text { CTGAGAAGAAGACCAGCGGC } \\
\text { GAAGAGCAGGGAGTAGGGCA }\end{array}$ & 107 & 6.25 & 1.9124 \\
\hline Exon 41 & $\begin{array}{l}\text { qPCRgTSC2_8S } \\
\text { qPCRgTSC2_8A }\end{array}$ & $\begin{array}{l}\text { TCACAGGTGCATCATAGCCG } \\
\text { CCCATATTCCCTACCCGCTG } \\
\end{array}$ & 107 & 3.125 & 2.2134 \\
\hline \multicolumn{6}{|c|}{ GADPH } \\
\hline Intron 1 & $\begin{array}{l}\text { gGAPDH_1S } \\
\text { gGAPDH_1A }\end{array}$ & $\begin{array}{l}\text { GCTCCCACCTTTCTCATCC } \\
\text { CTGCAGCGTACTCCCCAC } \\
\end{array}$ & 147 & 6.25 & 2.0545 \\
\hline \multicolumn{6}{|c|}{ GFIIB } \\
\hline Exon 1 & $\begin{array}{l}\text { qPCRgGFI1B_1S } \\
\text { qPCRgGFI1B_1A } \\
\end{array}$ & $\begin{array}{l}\text { TATTGCGAGGCTGAGTATGATGG } \\
\text { TTCATATCCCACAGTTCGACTTCA }\end{array}$ & 96 & 3.125 & 1.9485 \\
\hline \multicolumn{6}{|c|}{ SPACA9 } \\
\hline Exon 4 & $\begin{array}{l}\text { qPCRgSPACA9_2S } \\
\text { qPCRgSPACA9_2A }\end{array}$ & $\begin{array}{l}\text { GAGGCCCGGAACCACTAC } \\
\text { GAGTGGGCGATCCATTCTTTC }\end{array}$ & 111 & 3.125 & 2.1000 \\
\hline \multicolumn{6}{|c|}{ NTHL1 } \\
\hline Exon 6 & $\begin{array}{l}\text { qPCRgNTHL1_1S } \\
\text { qPCRgNTHL1_1A } \\
\end{array}$ & $\begin{array}{l}\text { TCAATGGACTCTTGGTGGGC } \\
\text { AAAGCCACTTCACAGACGGT } \\
\end{array}$ & 163 & 6.25 & 2.1350 \\
\hline \multicolumn{6}{|c|}{ PKD1 } \\
\hline Exon 45 & $\begin{array}{l}\text { qPCRgPKD1_1S } \\
\text { qPCRgPKD1_1A } \\
\end{array}$ & $\begin{array}{l}\text { GGCTCTCTACCCTGTGTCCT } \\
\text { CGGAGAATAACAGCCCCCAG } \\
\end{array}$ & 112 & 3.125 & 2.1407 \\
\hline Exon 46 & $\begin{array}{l}\text { qPCRgPKD1_2S } \\
\text { gPCRgPKD1_2A }\end{array}$ & $\begin{array}{l}\text { TAGGTGTGGTGGCGTTATGG } \\
\text { CTCTGGGGTGATGAGAGTGC }\end{array}$ & 107 & 6.25 & 2.1537 \\
\hline
\end{tabular}

Supplementary Table II-6: Q-PCR cycle program.

\begin{tabular}{ccccc}
\hline \multicolumn{2}{c}{ Cycling } & Time & Temperature & Ramp \\
\hline \multirow{2}{*}{ Holding } & $1 \mathrm{X}$ & $2 \mathrm{~min}$ & $50^{\circ} \mathrm{C}$ & $100 \%$ \\
\cline { 2 - 5 } & $1 \mathrm{X}$ & $10 \mathrm{~min}$ & $95^{\circ} \mathrm{C}$ & $100 \%$ \\
\hline \multirow{2}{*}{ Cycling } & $35 \mathrm{X}$ & $15 \mathrm{seg}$ & $95^{\circ} \mathrm{C}$ & $100 \%$ \\
\cline { 2 - 5 } & & $1 \mathrm{~min}$ & $60^{\circ} \mathrm{C}$ & $100 \%$ \\
\hline \multirow{3}{*}{ Melting } & $1 \mathrm{X}$ & $15 \mathrm{seg}$ & $95^{\circ} \mathrm{C}$ & $100 \%$ \\
\cline { 2 - 5 } & $1 \mathrm{X}$ & $1 \mathrm{~min}$ & $60^{\circ} \mathrm{C}$ & $100 \%$ \\
\cline { 2 - 5 } & $1 \mathrm{X}$ & $30 \mathrm{seg}$ & $95^{\circ} \mathrm{C}$ & $1 \%$ \\
\cline { 2 - 5 } & $1 \mathrm{X}$ & $15 \mathrm{seg}$ & $60^{\circ} \mathrm{C}$ & $100 \%$ \\
\hline
\end{tabular}


Supplementary Table II-7: List of softwares and websites consulted.

\begin{tabular}{|c|c|}
\hline Program & Web site \\
\hline Primer3 & http://primer3.ut.ee/ \\
\hline Primer-BLAST & https://www.ncbi.nlm.nih.gov/tools/primer-blast/ \\
\hline BLAST & https://blast.ncbi.nlm.nih.gov/Blast.cgi \\
\hline Beacon Designer Free Edition & http://www.premierbiosoft.com/qOligo/Oligo.jsp?PID=1 \\
\hline BLAT & http://genome.ucsc.edu/cgi-bin/hgBlat?command=start \\
\hline Human Genome Variation Society & http://varnomen.hgvs.org/ \\
\hline Variant Validator & https://variantvalidator.org/ \\
\hline Mutalyzer & http://www.lovd.nl/mutalyzer/ \\
\hline Leiden Open Variation Database TSC1 & http://chromium.lovd.n1/LOVD2/TSC/home.php?select_db=TSC1 \\
\hline Leiden Open Variation Database TSC2 & http://chromium.lovd.n1/LOVD2/TSC/home.php?select_db=TSC2 \\
\hline Ensembl & http://www.ensembl.org/index.html \\
\hline PolyPhen-2 & http://genetics.bwh.harvard.edu/pph2/ \\
\hline Mutation Taster & http://www.mutationtaster.org/ \\
\hline SIFT and PROVEAN & http://provean.jcvi.org/genome_submit_2.php \\
\hline PhosphositePlus & $\begin{array}{l}\text { https://www.phosphosite.org/homeAction.action?jsessionid=D937EFB } \\
\text { 1B37CAF55C7289FE52C813AA1 }\end{array}$ \\
\hline Acescan2 & http://genes.mit.edu/acescan2/index.html \\
\hline SpliceAid2 & http://193.206.120.249/splicing_tissue.html \\
\hline Human Splicing Finder 3.1 & http://www.umd.be/HSF3/ \\
\hline RepeatMasker & http://www.repeatmasker.org/cgi-bin/WEBRepeatMasker \\
\hline UniProtKB BLAST & https://www.uniprot.org/help/uniprotkb \\
\hline
\end{tabular}


Supplementary Table II-8: Primer pairs for function assessment of splicing variant.

\begin{tabular}{lll}
\hline \multicolumn{1}{c}{ Segment } & \multicolumn{1}{c}{ Name } & \multicolumn{1}{c}{ Oligonucleotide sequence (5' -3') } \\
\hline \multirow{2}{*}{ Mini-gene Exon 12-14 } & gTSC2_51S & GCCCATGGGGACCACCTTGGACAGCCCGGAGC \\
& gTSC2_50A & TCTAGAACTCGAGCTCATAGAACTGCCTGTTGATGAGC \\
\hline \multirow{2}{*}{ Mini-gene Exon 13-35 } & gTSC2_53S & GCCCATGGGGATCCACTCCGCCGTGGTCATGGAG \\
& gTSC2_52A & TCTAGAAGCGGCCGCCTCATTGGGCAGCAGGATTGGC \\
\hline \multirow{2}{*}{ Endogenous Exons 8-10 } & cTSC2_2S & TGGTCTGCTACAACTGCCTG \\
& cTSC2_1A & CGGCGAGTTCCTGAGAGAAT \\
\hline $\begin{array}{l}\text { BamHI restriction site: GGATTC } \\
\text { XhoI restriction site: CTCGAG } \\
\text { NotI restriction site: GCGGCCGC }\end{array}$ & &
\end{tabular}

Supplementary Table II-9: Rules for combining criteria to classify sequence variants according to ACMG (Richards, Aziz et al. 2015).

Rules for combining criteria to classify sequence variants

\begin{tabular}{|c|c|c|}
\hline \multirow[t]{3}{*}{ Pathogenic } & (i) 1 Very strong (PVS1) AND & $\begin{array}{l}\text { (a) } \geq 1 \text { Strong (PS1-PS4) OR } \\
\text { (b) } \geq 2 \text { Moderate (PM1-PM6) OR } \\
\text { (c) } 1 \text { Moderate (PM1-PM6) and } 1 \text { supporting (PP1-PP5) OR } \\
\text { (d) } \geq 2 \text { Supporting (PP1-PP5) OR }\end{array}$ \\
\hline & \multicolumn{2}{|l|}{ (ii) $\geq 2$ Strong (PS1-PS4) OR } \\
\hline & (iii) 1 Strong (PS1-PS4) AND & $\begin{array}{l}\text { (a) } \geq 3 \text { Moderate (PM1-PM6) OR } \\
\text { (b) } 2 \text { Moderate (PM1-PM6) AND } \geq 2 \text { Supporting (PP1-PP5) OR } \\
\text { (c)1 Moderate (PM1-PM6) AND } \geq 4 \text { supporting (PP1-PP5). }\end{array}$ \\
\hline Likely pathogenic & \multicolumn{2}{|c|}{$\begin{array}{l}\text { (i) } 1 \text { Very strong (PVS1) AND } 1 \text { moderate (PM1-PM6) OR } \\
\text { (ii) } 1 \text { Strong (PS1-PS4) AND } 1-2 \text { moderate (PM1-PM6) OR } \\
\text { (iii) } 1 \text { Strong (PS1-PS4) AND } \geq 2 \text { supporting (PP1-PP5) OR } \\
\text { (iv) } \geq 3 \text { Moderate (PM1-PM6) OR } \\
\text { (v) } 2 \text { Moderate (PM1-PM6) AND } \geq 2 \text { supporting (PP1-PP5) OR } \\
\text { (vi) } 1 \text { Moderate (PM1-PM6) AND } \geq 4 \text { supporting (PP1-PP5) }\end{array}$} \\
\hline Benign & \multicolumn{2}{|l|}{$\begin{array}{l}\text { (i) } 1 \text { Stand-alone (BA1) OR } \\
\text { (ii) } \geq 2 \text { Strong (BS1-BS4). }\end{array}$} \\
\hline Likely benign & \multicolumn{2}{|c|}{$\begin{array}{l}\text { (i) } 1 \text { Strong (BS1-BS4) and } 1 \text { supporting (BP1-BP7) OR } \\
\text { (ii) } \geq 2 \text { Supporting (BP1-BP7). }\end{array}$} \\
\hline Uncertain significance & \multicolumn{2}{|c|}{$\begin{array}{l}\text { (i) Other criteria shown above are not met OR } \\
\text { (ii) the criteria for benign and pathogenic are contradictory. }\end{array}$} \\
\hline
\end{tabular}


Supplementary Table II-10: Evidence of benign impact according to ACMG (Richards, Aziz et al. 2015).

Evidence of benign impact

这

BA1: Allele frequency is $>5 \%$ in Exome Sequencing Project, 1000 Genomes Project, or Exome Aggregation Consortium

BS1: Allele frequency is greater than expected for disorder (see Table 6)

20 BS2: Observed in a healthy adult individual for a recessive (homozygous), dominant (heterozygous), or X-linked (hemizygous)

disorder, with full penetrance expected at an early age

क BS3: Well-established in vitro or in vivo functional studies show no damaging effect on protein function or splicing BS4: Lack of segregation in affected members of a family

BP1: Missense variant in a gene for which primarily truncating variants are known to cause disease

BP2: Observed in trans with a pathogenic variant for a fully penetrant dominant gene/disorder or observed in cis with a pathogenic variant in any inheritance pattern

vo BP3: In-frame deletions/insertions in a repetitive region without a known function

BP4: Multiple lines of computational evidence suggest no impact on gene or gene product (conservation, evolutionary, splicing

impact, etc.)

BP5: Variant found in a case with an alternate molecular basis for disease

B BP6 Reputable source recently reports variant as benign, but the evidence is not available to the laboratory to perform an independent evaluation

BP7 A synonymous (silent) variant for which splicing prediction algorithms predict no impact to the splice consensus sequence nor the creation of a new splice site AND the nucleotide is not highly conserved 
Supplementary Table II-11: TSC1 and TSC2 exonic single nucleotide variants.

1000 Genomes

Variant Exon Existing variation

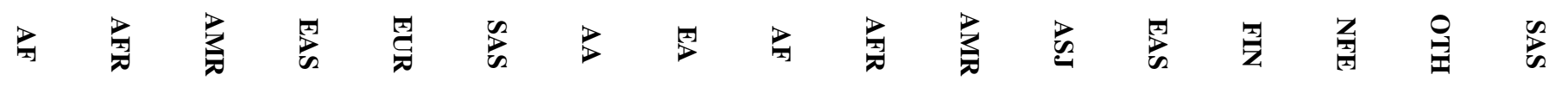

\begin{tabular}{|c|c|c|c|c|c|c|c|c|c|c|c|c|c|c|c|c|c|c|c|}
\hline & & & & & & & & TSC1 & & & & & & & & & & & \\
\hline c. $-99 \mathrm{C}>\mathrm{T}$ & $02 / 23$ & rs114755636 & 0.004 & 0.015 & 0.001 & 0.000 & 0.000 & 0.000 & - & - & - & - & - & - & - & - & - & - & - \\
\hline c. $-129 A>T$ & $02 / 23$ & rs116951280 & 0.005 & 0.000 & 0.013 & 0.000 & 0.014 & 0.001 & - & - & - & - & - & - & - & - & - & - & - \\
\hline $\begin{array}{l}\text { c. } 840 A>G \\
(\text { p. }=)\end{array}$ & $09 / 23$ & rs1171852730 & - & - & - & - & - & - & - & - & 0.000 & 0.000 & 0.000 & 0.000 & 0.000 & 0.000 & 0.000 & 0.000 & 0.000 \\
\hline $\begin{array}{l}\text { c. } 965 \mathrm{~T}>\mathrm{C} \\
\text { (p.Met322Thr) }\end{array}$ & $10 / 23$ & rs1073123 & 0.135 & 0.234 & 0.094 & 0.074 & 0.131 & 0.098 & 0.220 & 0.138 & 0.125 & 0.217 & 0.086 & 0.141 & 0.088 & 0.102 & 0.138 & 0.126 & 0.104 \\
\hline $\begin{array}{l}\text { c. } 1342 C>T \\
\text { (p.Pro448Ser) }\end{array}$ & $14 / 23$ & rs118203518 & 0.004 & 0.015 & 0.001 & 0.000 & 0.000 & 0.000 & 0.011 & 0.000 & 0.001 & 0.011 & 0.001 & 0.000 & 0.000 & 0.000 & 0.000 & 0.000 & 0.000 \\
\hline $\begin{array}{l}\text { c. } 1335 \mathrm{~A}>\mathrm{G} \\
\text { (p.=) }\end{array}$ & $14 / 23$ & rs7862221 & 0.139 & 0.233 & 0.097 & 0.074 & 0.144 & 0.101 & 0.219 & 0.146 & 0.132 & 0.218 & 0.088 & 0.150 & 0.088 & 0.115 & 0.148 & 0.135 & 0.107 \\
\hline $\begin{array}{l}\text { c. } 1760 A>G \\
\text { (p.Lys587Arg) }\end{array}$ & $15 / 23$ & rs118203576 & 0.018 & 0.000 & 0.128 & 0.000 & 0.002 & 0.000 & 0.002 & 0.000 & 0.026 & 0.002 & 0.175 & 0.000 & 0.000 & 0.012 & 0.000 & 0.026 & 0.001 \\
\hline $\begin{array}{l}\text { c. } 2829 \mathrm{C}>\mathrm{T} \\
(\mathrm{p} .=)\end{array}$ & $22 / 23$ & rs4962081 & 0.062 & 0.095 & 0.097 & 0.023 & 0.064 & 0.032 & 0.091 & 0.082 & 0.079 & 0.089 & 0.154 & 0.053 & 0.028 & 0.046 & 0.081 & 0.076 & 0.051 \\
\hline c. $* 4437 \mathrm{G}>\mathrm{A}$ & $23 / 23$ & rs 11553763 & 0.103 & 0.214 & 0.123 & 0.040 & 0.068 & 0.043 & - & - & - & - & - & - & - & - & - & - & - \\
\hline \multirow[t]{2}{*}{ c. $* 3679 \mathrm{G}>\mathrm{A}$} & $23 / 23$ & rs 1050700 & 0.726 & 0.595 & 0.648 & 0.825 & 0.749 & 0.832 & - & - & - & - & - & - & - & - & - & - & - \\
\hline & $23 / 23$ & rs739441 & - & 0.660 & 0.752 & 0.821 & 0.830 & 0.834 & - & - & - & - & - & - & - & - & - & - & - \\
\hline c. $* 1488 \mathrm{C}>\mathrm{T}$ & $23 / 23$ & rs739442 & 0.451 & 0.056 & 0.496 & 0.581 & 0.612 & 0.651 & - & - & - & - & - & - & - & - & - & - & - \\
\hline c. $* 1362 \mathrm{G}>\mathrm{T}$ & $23 / 23$ & - & - & - & - & - & - & - & - & - & - & - & - & - & - & - & - & - & - \\
\hline c. $* 1275 \mathrm{~T}>\mathrm{G}$ & $23 / 23$ & rs 2809244 & - & 0.778 & 0.738 & 0.682 & 0.753 & 0.780 & - & - & - & - & - & - & - & - & - & - & - \\
\hline c. $* 289 \mathrm{delT}$ & $23 / 23$ & rs 11323835 & 0.439 & 0.137 & 0.473 & 0.566 & 0.515 & 0.616 & - & - & - & - & - & - & - & - & - & - & - \\
\hline c.*191dupC & $23 / 23$ & - & - & - & - & - & - & - & - & - & - & - & - & - & - & - & - & - & - \\
\hline c. $* 107 \mathrm{~T}>\mathrm{C}$ & $23 / 23$ & rs116917669 & 0.017 & 0.002 & 0.020 & 0.002 & 0.044 & 0.024 & - & - & - & - & - & - & - & - & - & - & - \\
\hline c. $* 1 \mathrm{G}>\mathrm{A}$ & $23 / 23$ & - & - & - & - & - & - & - & - & - & - & - & - & - & - & - & - & - & - \\
\hline $\begin{array}{l}\text { c. } 3324 \mathrm{C}>\mathrm{T} \\
(\mathrm{p} .=)\end{array}$ & $23 / 23$ & rs 35593170 & 0.016 & 0.003 & 0.095 & 0.006 & 0.002 & 0.001 & 0.001 & 0.001 & 0.018 & 0.002 & 0.125 & 0.000 & 0.003 & 0.002 & 0.001 & 0.013 & 0.001 \\
\hline $\begin{array}{l}\text { c. } 3282 G>A \\
(p .=)\end{array}$ & $23 / 23$ & rs116747861 & 0.005 & 0.017 & 0.001 & 0.000 & 0.000 & 0.000 & 0.013 & 0.001 & 0.002 & 0.014 & 0.002 & 0.005 & 0.000 & 0.000 & 0.001 & 0.002 & 0.000 \\
\hline
\end{tabular}




\begin{tabular}{|c|c|c|c|c|c|c|c|c|c|c|c|c|c|c|c|c|c|c|c|}
\hline $\begin{array}{l}\text { c.3127_3129del } \\
\text { (p.Ser1043del) }\end{array}$ & $23 / 23$ & rs397514812 & - & - & - & - & - & $\overline{-}$ & - & - & 0.000 & 0.000 & 0.000 & 0.000 & 0.000 & 0.000 & 0.000 & 0.000 & 0.000 \\
\hline \multicolumn{20}{|c|}{ TSC2 } \\
\hline $\begin{array}{l}\text { c.52C>G } \\
\text { (p.Leu18Val) }\end{array}$ & $02 / 42$ & - & - & - & - & - & - & - & - & - & - & - & - & - & - & - & - & - & - \\
\hline $\begin{array}{l}\text { c. } 499 \mathrm{~T}>\mathrm{C} \\
\text { (p.Trp167Arg) }\end{array}$ & $06 / 42$ & - & - & - & - & - & - & - & - & - & - & - & - & - & - & - & - & - & - \\
\hline $\begin{array}{l}\text { c. } 881 \mathrm{G}>\mathrm{A} \\
\text { (p.Gly294Glu) }\end{array}$ & $10 / 42$ & rs45487497 & - & - & - & - & - & - & - & - & - & - & - & - & - & - & - & - & - \\
\hline $\begin{array}{l}\text { c. } 1100 \mathrm{G}>\mathrm{A} \\
\text { (p.Arg367Gln) }\end{array}$ & $11 / 42$ & rs1800725 & 0.009 & 0.002 & 0.016 & 0.000 & 0.014 & 0.017 & 0.005 & 0.020 & 0.014 & 0.003 & 0.011 & 0.027 & 0.000 & 0.007 & 0.018 & 0.015 & 0.017 \\
\hline $\begin{array}{l}\text { c. } 1443 \mathrm{G}>\mathrm{T} \\
\text { (p.Glu481Asp) }\end{array}$ & $14 / 42$ & CS091000 & - & - & - & - & - & - & - & - & - & - & - & - & - & - & - & - & - \\
\hline $\begin{array}{l}\text { c. } 1578 \mathrm{C}>\mathrm{T} \\
\text { (p.=) }\end{array}$ & $15 / 42$ & rs34012042, & 0.035 & 0.002 & 0.046 & 0.000 & 0.073 & 0.070 & 0.015 & 0.070 & 0.057 & 0.011 & 0.033 & 0.100 & 0.000 & 0.086 & 0.067 & 0.064 & 0.070 \\
\hline $\begin{array}{l}c .1731 \mathrm{C}>\mathrm{T} \\
(\mathrm{p} .=)\end{array}$ & $17 / 42$ & rs 144122318 & 0.000 & 0.000 & 0.000 & 0.000 & 0.001 & 0.000 & - & - & 0.000 & 0.000 & 0.000 & 0.000 & 0.000 & 0.000 & 0.000 & 0.000 & 0.000 \\
\hline $\begin{array}{l}\text { c. } 2073 \mathrm{C}>\mathrm{T} \\
(\mathrm{p} .=)\end{array}$ & $19 / 42$ & rs 45512398 & 0.000 & 0.001 & 0.000 & 0.001 & 0.000 & 0.000 & 0.000 & 0.001 & 0.001 & 0.000 & 0.000 & 0.011 & 0.000 & 0.000 & 0.000 & 0.001 & 0.001 \\
\hline $\begin{array}{l}\text { c.2580T }>C \\
(\text { p. }=)\end{array}$ & $23 / 42$ & rs 13337626 & 0.063 & 0.093 & 0.042 & 0.002 & 0.088 & 0.074 & 0.087 & 0.086 & 0.070 & 0.088 & 0.035 & 0.077 & 0.001 & 0.111 & 0.082 & 0.069 & 0.063 \\
\hline $\begin{array}{l}\text { c. } 3126 \mathrm{G}>\mathrm{C} \\
(\text { p. }=)\end{array}$ & $27 / 42$ & rs 36078782 & 0.013 & 0.047 & 0.003 & 0.000 & 0.000 & 0.000 & 0.037 & 0.000 & 0.003 & 0.043 & 0.003 & 0.000 & 0.000 & 0.000 & 0.000 & 0.003 & 0.000 \\
\hline $\begin{array}{l}\text { c.3431T>A } \\
\text { (p.Val1144Glu) }\end{array}$ & $30 / 42$ & - & - & - & - & - & - & - & - & - & - & - & - & - & - & - & - & - & - \\
\hline $\begin{array}{l}\text { c.3557A>G } \\
\text { (p.Tyr1186Cys) }\end{array}$ & $30 / 42$ & rs 137854421 & 0.000 & 0.001 & 0.000 & 0.000 & 0.000 & 0.000 & 0.000 & 0.000 & 0.000 & 0.000 & 0.000 & 0.000 & 0.000 & 0.000 & 0.000 & 0.000 & 0.001 \\
\hline $\begin{array}{l}\text { c. } 3768 \mathrm{G}>A \\
(\mathrm{p} .=)\end{array}$ & $31 / 42$ & rs201599540 & 0.000 & 0.000 & 0.000 & 0.001 & 0.000 & 0.000 & - & - & 0.000 & 0.000 & 0.000 & 0.000 & 0.000 & 0.000 & 0.000 & 0.000 & 0.000 \\
\hline $\begin{array}{l}\text { c.3889G >A } \\
\text { (p.Ala1297Thr) }\end{array}$ & $33 / 42$ & rs 45517319 & 0.001 & 0.000 & 0.000 & 0.000 & 0.003 & 0.002 & 0.002 & 0.004 & 0.003 & 0.001 & 0.001 & 0.004 & 0.000 & 0.005 & 0.004 & 0.002 & 0.002 \\
\hline $\begin{array}{l}\text { c.3914C }>\text { T } \\
\text { (p.Pro1305Leu) }\end{array}$ & $33 / 42$ & rs 45517320 & 0.009 & 0.033 & 0.003 & 0.000 & 0.000 & 0.000 & 0.021 & 0.000 & 0.002 & 0.026 & 0.002 & 0.000 & 0.000 & 0.000 & 0.000 & 0.000 & 0.000 \\
\hline $\begin{array}{l}\text { c. } 3915 G>A \\
(p .=)\end{array}$ & $33 / 42$ & rs 11551373 & 0.022 & 0.077 & 0.006 & 0.001 & 0.002 & 0.000 & 0.066 & 0.000 & 0.005 & 0.068 & 0.004 & 0.000 & 0.000 & 0.000 & 0.000 & 0.002 & 0.000 \\
\hline $\begin{array}{l}\text { c.3986G >A } \\
\text { (p.Arg1329His) }\end{array}$ & $33 / 42$ & rs45517323, & 0.020 & 0.070 & 0.007 & 0.001 & 0.002 & 0.000 & 0.048 & 0.001 & 0.005 & 0.054 & 0.002 & 0.001 & 0.001 & 0.011 & 0.001 & 0.004 & 0.000 \\
\hline $\begin{array}{l}\text { c.4316G >A } \\
\text { (p.Gly1439Asp) }\end{array}$ & $34 / 42$ & rs 150397923 & 0.005 & 0.020 & 0.000 & 0.000 & 0.000 & 0.000 & 0.013 & 0.000 & 0.001 & 0.017 & 0.001 & 0.000 & 0.000 & 0.000 & 0.000 & 0.000 & 0.000 \\
\hline $\begin{array}{l}c .4536 C>T \\
(p .=)\end{array}$ & $35 / 42$ & rs 35986575 & 0.000 & 0.000 & 0.001 & 0.000 & 0.000 & 0.000 & 0.001 & 0.005 & 0.003 & 0.000 & 0.001 & 0.021 & 0.000 & 0.002 & 0.004 & 0.005 & 0.002 \\
\hline c. $4716 \mathrm{G}>\mathrm{T}$ & $37 / 42$ & - & - & - & - & - & - & _- & - & - & - & - & - & - & - & - & - & - & - \\
\hline
\end{tabular}




\begin{tabular}{|c|c|c|c|c|c|c|c|c|c|c|c|c|c|c|c|c|c|c|c|}
\hline$(\mathrm{p} .=)$ & & & & & & & & & & & & & & & & & & & \\
\hline $\begin{array}{l}\mathrm{c} .4908 \mathrm{C}>\mathrm{T} \\
(\mathrm{p} .=)\end{array}$ & $38 / 42$ & rs115200071 & 0.002 & 0.008 & 0.000 & 0.000 & 0.000 & 0.000 & 0.007 & 0.001 & 0.001 & 0.007 & 0.000 & 0.000 & 0.000 & 0.000 & 0.000 & 0.000 & 0.000 \\
\hline $\begin{array}{l}\text { c. } 4959 \mathrm{C}>\mathrm{T} \\
(\mathrm{p} .=)\end{array}$ & $38 / 42$ & rs 45517384 & 0.003 & 0.002 & 0.009 & 0.000 & 0.008 & 0.000 & 0.003 & 0.015 & 0.010 & 0.003 & 0.007 & 0.001 & 0.000 & 0.020 & 0.014 & 0.010 & 0.001 \\
\hline $\begin{array}{l}\text { c.4982C>T } \\
\text { (p.Thr1661Ile) }\end{array}$ & $38 / 42$ & rs776541842 & - & - & - & - & - & - & - & - & 0.000 & 0.000 & 0.000 & 0.000 & 0.000 & 0.000 & 0.000 & 0.000 & 0.000 \\
\hline $\begin{array}{l}\text { c. } .5010 C>T \\
(p .=)\end{array}$ & $39 / 42$ & rs376306544 & - & - & - & - & - & - & 0.000 & 0.000 & 0.000 & 0.000 & 0.000 & 0.000 & 0.000 & 0.000 & 0.000 & 0.000 & 0.000 \\
\hline $\begin{array}{l}\text { c. } 5025 \mathrm{G}>A \\
(\mathrm{p} .=)\end{array}$ & $39 / 42$ & rs 35118875 & 0.004 & 0.014 & 0.003 & 0.000 & 0.000 & 0.000 & 0.016 & 0.000 & 0.001 & 0.014 & 0.001 & 0.000 & 0.000 & 0.000 & 0.000 & 0.001 & 0.000 \\
\hline $\begin{array}{l}\text { c. } 5051 \mathrm{C}>\mathrm{T} \\
\text { (p.Ser1684Phe) }\end{array}$ & $39 / 42$ & - & - & - & - & - & - & - & - & - & - & - & - & - & - & - & - & - & - \\
\hline $\begin{array}{l}\text { c. } 5202 \mathrm{~T}>\mathrm{C} \\
(\mathrm{p} .=)\end{array}$ & $41 / 42$ & rs 1748 & 0.276 & 0.748 & 0.199 & 0.000 & 0.184 & 0.074 & 0.634 & 0.178 & 0.178 & 0.662 & 0.112 & 0.210 & 0.000 & 0.207 & 0.177 & 0.179 & 0.081 \\
\hline $\begin{array}{l}\text { c. } 5312 \mathrm{C}>\mathrm{T} \\
\text { (p.Pro1771Leu) }\end{array}$ & $42 / 42$ & rs 137854214 & 0.001 & 0.004 & 0.000 & 0.000 & 0.000 & 0.000 & 0.001 & 0.000 & 0.000 & 0.002 & 0.000 & 0.000 & 0.000 & 0.000 & 0.000 & 0.000 & 0.000 \\
\hline $\begin{array}{l}\text { c.5397G }>C \\
(p .=)\end{array}$ & $42 / 42$ & rs 1051771 & 0.041 & 0.002 & 0.055 & 0.000 & 0.101 & 0.064 & 0.019 & 0.099 & 0.073 & 0.015 & 0.033 & 0.094 & 0.000 & 0.144 & 0.096 & 0.075 & 0.058 \\
\hline c. $* 26 \mathrm{G}>\mathrm{A}$ & $42 / 42$ & rs 13332015 & 0.039 & 0.138 & 0.016 & 0.000 & 0.000 & 0.000 & 0.110 & 0.000 & 0.009 & 0.122 & 0.006 & 0.000 & 0.000 & 0.000 & 0.000 & 0.005 & 0.000 \\
\hline c. $* 35 \mathrm{G}>\mathrm{A}$ & $42 / 42$ & rs200025534 & 0.002 & 0.006 & 0.000 & 0.000 & 0.000 & 0.000 & 0.008 & 0.000 & 0.001 & 0.008 & 0.000 & 0.000 & 0.000 & 0.000 & 0.000 & 0.001 & 0.000 \\
\hline c. $* 61 \_* 62$ delAA & $42 / 42$ & rs 36032671 & - & - & - & - & - & - & - & - & - & - & - & - & - & - & - & - & - \\
\hline
\end{tabular}


Supplementary Table II-12: TSC1 and TSC2 intronic single nucleotide variants.

1000 Genomes

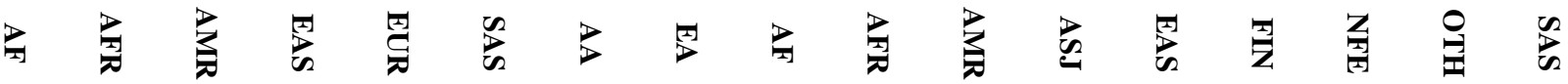

\section{TSC1}

\begin{tabular}{|c|c|c|c|c|c|c|c|c|c|c|c|c|c|c|c|c|c|c|c|}
\hline c. $2976-43 G>C$ & $22 / 22$ & rs7853849 & 0.025 & 0.084 & 0.016 & 0.000 & 0.003 & 0.000 & 0.066 & 0.001 & 0.007 & 0.077 & 0.009 & 0.000 & 0.000 & 0.000 & 0.001 & 0.008 & 0.000 \\
\hline c. $2976-54 \mathrm{G}>\mathrm{A}$ & $22 / 22$ & rs118203740 & 0.007 & 0.019 & 0.007 & 0.000 & 0.003 & 0.000 & - & - & - & - & - & - & - & - & - & - & - \\
\hline c. $2625+68 \mathrm{G}>\mathrm{A}$ & $20 / 22$ & rs 1076160 & 0.412 & 0.055 & 0.450 & 0.559 & 0.511 & 0.618 & - & - & - & - & - & - & - & - & - & - & - \\
\hline c. $2502+131 \mathrm{C}>\mathrm{T}$ & $19 / 22$ & rs7020175 & 0.082 & 0.107 & 0.059 & 0.072 & 0.086 & 0.073 & - & - & - & - & - & - & - & - & - & - & - \\
\hline c. $2502+51 \mathrm{~A}>\mathrm{G}$ & $19 / 22$ & rs75802666 & 0.080 & 0.004 & 0.042 & 0.198 & 0.067 & 0.099 & 0.009 & 0.050 & 0.073 & 0.009 & 0.079 & 0.024 & 0.197 & 0.124 & 0.054 & 0.067 & 0.080 \\
\hline c. $2392-35 \mathrm{~T}>\mathrm{C}$ & $18 / 22$ & rs11243931 & 0.175 & 0.363 & 0.108 & 0.074 & 0.146 & 0.101 & 0.346 & 0.148 & 0.142 & 0.348 & 0.094 & 0.150 & 0.088 & 0.117 & 0.149 & 0.138 & 0.107 \\
\hline c. $2392-222 \mathrm{C}>\mathrm{T}$ & $18 / 22$ & rs141011030 & 0.005 & 0.000 & 0.013 & 0.000 & 0.014 & 0.000 & - & - & - & - & - & - & - & - & - & - & - \\
\hline c. $2391+59 \mathrm{G}>\mathrm{C}$ & $18 / 22$ & rs117301441 & 0.003 & 0.000 & 0.004 & 0.000 & 0.012 & 0.000 & - & - & - & - & - & - & - & - & - & - & - \\
\hline c. $2391+34 \mathrm{G}>\mathrm{A}$ & $18 / 22$ & rs116518821 & 0.010 & 0.027 & 0.013 & 0.001 & 0.004 & 0.000 & 0.022 & 0.003 & 0.004 & 0.025 & 0.008 & 0.017 & 0.000 & 0.000 & 0.002 & 0.008 & 0.000 \\
\hline c. $2041+268 A>G$ & $16 / 22$ & rs12345576 & 0.175 & 0.364 & 0.108 & 0.074 & 0.146 & 0.101 & - & - & - & - & - & - & - & - & - & - & - \\
\hline c. $1439-37 \mathrm{C}>\mathrm{T}$ & $14 / 22$ & rs10901220 & 0.139 & 0.234 & 0.097 & 0.074 & 0.144 & 0.101 & 0.204 & 0.132 & 0.133 & 0.224 & 0.090 & 0.152 & 0.090 & 0.118 & 0.153 & 0.135 & 0.107 \\
\hline c. $1334-55 C>G$ & $13 / 22$ & rs 7872606 & 0.082 & 0.106 & 0.059 & 0.072 & 0.086 & 0.073 & - & - & - & - & - & - & - & - & - & - & - \\
\hline c. $1334-174 \mathrm{~T}>\mathrm{C}$ & $13 / 22$ & rs117147322 & 0.017 & 0.002 & 0.020 & 0.001 & 0.042 & 0.024 & - & - & - & - & - & - & - & - & - & - & - \\
\hline c. $1333+209 \mathrm{C}>\mathrm{T}$ & $13 / 22$ & rs 7872860 & 0.139 & 0.235 & 0.097 & 0.074 & 0.144 & 0.101 & - & - & - & - & - & - & - & - & - & - & - \\
\hline c. $1333+187 \mathrm{~A}>\mathrm{G}$ & $13 / 22$ & rs114683952 & 0.010 & 0.027 & 0.013 & 0.000 & 0.004 & 0.000 & - & - & - & - & - & - & - & - & - & - & - \\
\hline c.1142-26_1142-25del & $11 / 22$ & - & - & - & - & - & - & - & - & - & - & - & - & - & - & - & - & - & - \\
\hline c. $1142-33 A>G$ & $11 / 22$ & rs6597586 & 0.135 & 0.232 & 0.094 & 0.074 & 0.131 & 0.098 & 0.218 & 0.138 & 0.124 & 0.217 & 0.086 & 0.142 & 0.088 & 0.102 & 0.138 & 0.126 & 0.103 \\
\hline c. $1141+63 \mathrm{G}>\mathrm{A}$ & $11 / 22$ & rs77891974 & 0.011 & 0.038 & 0.006 & 0.000 & 0.000 & 0.000 & - & - & - & - & - & - & - & - & - & - & - \\
\hline c. $738-197 \mathrm{~T}>\mathrm{A}$ & $08 / 22$ & rs1336366611 & - & - & - & - & - & - & - & - & - & - & - & - & - & - & - & - & - \\
\hline c. $-80-71 \mathrm{~T}>\mathrm{A}$ & $02 / 22$ & rs45607538 & 0.001 & 0.000 & 0.004 & 0.000 & 0.002 & 0.001 & - & - & - & - & - & - & - & - & - & - & - \\
\hline \multicolumn{20}{|c|}{ TSC2 } \\
\hline c. $226-101 \mathrm{C}>\mathrm{T}$ & $03 / 41$ & rs144408387 & 0.008 & 0.002 & 0.027 & 0.000 & 0.017 & 0.001 & - & - & - & - & - & - & - & - & - & - & - \\
\hline
\end{tabular}




\begin{tabular}{|c|c|c|c|c|c|c|c|c|c|c|c|c|c|c|c|c|c|c|c|}
\hline c. $226-82 C>T$ & $03 / 41$ & rs7185742 & 0.003 & 0.000 & 0.003 & 0.001 & 0.011 & 0.003 & - & - & - & - & - & - & - & - & - & - & - \\
\hline c. $336+33 \mathrm{G}>\mathrm{T}$ & $04 / 41$ & rs45517104 & 0.009 & 0.034 & 0.001 & 0.000 & 0.000 & 0.000 & 0.033 & 0.000 & 0.002 & 0.040 & 0.001 & 0.000 & 0.000 & 0.000 & 0.000 & 0.002 & 0.000 \\
\hline c. $336+66 \mathrm{G}>\mathrm{C}$ & $04 / 41$ & rs920782009 & - & - & - & - & - & - & - & - & - & - & - & - & - & - & - & - & - \\
\hline c. $482-114 \mathrm{~T}>\mathrm{C}$ & $05 / 41$ & rs77037371 & 0.012 & 0.041 & 0.009 & 0.000 & 0.000 & 0.000 & - & - & - & - & - & - & - & - & - & - & - \\
\hline c. $482-68 \mathrm{C}>\mathrm{G}$ & $05 / 41$ & rs 2516734 & 0.153 & 0.374 & 0.151 & 0.000 & 0.136 & 0.032 & - & - & - & - & - & - & - & - & - & - & - \\
\hline c. $482-59 \mathrm{G}>\mathrm{A}$ & $05 / 41$ & rs200833855 & - & - & - & - & - & - & - & - & - & - & - & - & - & - & - & - & - \\
\hline c. $600-84 \mathrm{C}>\mathrm{T}$ & $06 / 41$ & rs35364892 & 0.007 & 0.008 & 0.001 & 0.000 & 0.003 & 0.020 & - & - & 0.009 & 0.007 & 0.003 & 0.036 & 0.000 & 0.006 & 0.008 & 0.010 & 0.019 \\
\hline c. $648+12 C>A$ & $07 / 41$ & - & - & - & - & - & - & - & - & - & - & - & - & - & - & - & - & - & - \\
\hline c. $848+113 A>G$ & $09 / 41$ & rs56077830 & 0.011 & 0.038 & 0.004 & 0.000 & 0.000 & 0.000 & - & - & - & - & - & - & - & - & - & - & - \\
\hline c. $848+143 \mathrm{G}>\mathrm{T}$ & $09 / 41$ & rs78160478 & 0.046 & 0.042 & 0.076 & 0.000 & 0.099 & 0.022 & - & - & - & - & - & - & - & - & - & - & - \\
\hline c. $976-100 \mathrm{C}>\mathrm{G}$ & $10 / 41$ & rs2074968 & - & 0.398 & 0.499 & 0.714 & 0.547 & 0.665 & - & - & - & - & - & - & - & - & - & - & - \\
\hline c. $976-63 \mathrm{G}>\mathrm{A}$ & $10 / 41$ & rs 12927333 & 0.035 & 0.002 & 0.046 & 0.000 & 0.075 & 0.064 & - & - & - & - & - & - & - & - & - & - & - \\
\hline c.1257+109dupG & $12 / 41$ & - & - & - & - & - & - & - & - & - & - & - & - & - & - & - & - & - & - \\
\hline c. $1258-223 \mathrm{G}>\mathrm{A}$ & $12 / 41$ & rs924724434 & - & - & - & - & - & - & - & - & - & - & - & - & - & - & - & - & - \\
\hline c. $1361+54 \_1361+57 \mathrm{del}$ & $13 / 41$ & rs137854304 & 0.001 & - & - & - & - & - & - & - & - & - & - & - & - & - & - & - & - \\
\hline c. $1362-151 \mathrm{G}>\mathrm{T}$ & $13 / 41$ & rs545796760 & 0.000 & 0.000 & 0.000 & 0.000 & 0.002 & 0.000 & - & - & - & - & - & - & - & - & - & - & - \\
\hline c. $1443+65 \mathrm{~T}>\mathrm{G}$ & $14 / 41$ & rs115599333 & 0.007 & 0.027 & 0.000 & 0.000 & 0.000 & 0.000 & - & - & - & - & - & - & - & - & - & - & - \\
\hline c. $1444-201 G>A$ & $14 / 41$ & rs60763712 & 0.051 & 0.181 & 0.017 & 0.000 & 0.002 & 0.000 & - & - & - & - & - & - & - & - & - & - & - \\
\hline c. $1599+85 \_1599+87 \mathrm{del}$ & $15 / 41$ & - & - & - & - & - & - & - & - & - & - & - & - & - & - & - & - & - & - \\
\hline c. $1599+162 C>T$ & $15 / 41$ & rs74819416 & 0.009 & 0.030 & 0.006 & 0.000 & 0.000 & 0.000 & - & - & - & - & - & - & - & - & - & - & - \\
\hline c. $1599+216 \mathrm{~T}>\mathrm{G}$ & $15 / 41$ & rs 17654678 & 0.038 & 0.003 & 0.042 & 0.001 & 0.112 & 0.044 & - & - & - & - & - & - & - & - & - & - & - \\
\hline c.1599+282_1599+283insCTGGGG & $15 / 41$ & - & - & - & - & - & - & - & - & - & - & - & - & - & - & - & - & - & - \\
\hline c. $1716+183 A>G$ & $16 / 41$ & rs8063461 & 0.615 & 0.350 & 0.716 & 0.839 & 0.590 & 0.698 & - & - & - & - & - & - & - & - & - & - & - \\
\hline c. $1717-121 \mathrm{G}>\mathrm{A}$ & $16 / 41$ & rs1275330311 & - & - & - & - & - & - & - & - & - & - & - & - & - & - & - & - & - \\
\hline c. $1717-55 \mathrm{~T}>\mathrm{C}$ & $16 / 41$ & rs7187438 & 0.393 & 0.714 & 0.297 & 0.221 & 0.371 & 0.229 & - & - & - & - & - & - & - & - & - & - & - \\
\hline c. $1840-172 \mathrm{G}>\mathrm{A}$ & $17 / 41$ & rs532002248 & 0.000 & 0.000 & 0.001 & 0.001 & 0.000 & 0.000 & - & - & - & - & - & - & - & - & - & - & - \\
\hline c. $1840-136 \mathrm{~T}>\mathrm{C}$ & $17 / 41$ & rs113749674 & 0.013 & 0.046 & 0.006 & 0.000 & 0.000 & 0.000 & - & - & - & - & - & - & - & - & - & - & - \\
\hline c. $2097+43 \mathrm{~A}>\mathrm{G}$ & $19 / 41$ & rs 186681035 & 0.003 & 0.001 & 0.001 & 0.000 & 0.010 & 0.001 & 0.001 & 0.010 & 0.009 & 0.003 & 0.003 & 0.012 & 0.000 & 0.026 & 0.013 & 0.009 & 0.005 \\
\hline c. $2098-114 A>G$ & $19 / 41$ & rs6600185 & 0.110 & 0.393 & 0.040 & 0.000 & 0.002 & 0.000 & - & - & - & - & - & - & - & - & - & - & - \\
\hline
\end{tabular}




\begin{tabular}{|c|c|c|c|c|c|c|c|c|c|c|c|c|c|c|c|c|c|c|c|}
\hline c. $2221-28 \mathrm{~A}>\mathrm{G}$ & $20 / 41$ & rs7196184 & 0.109 & 0.392 & 0.040 & 0.000 & 0.002 & 0.000 & 0.330 & 0.001 & 0.026 & 0.354 & 0.019 & 0.005 & 0.000 & 0.000 & 0.001 & 0.017 & 0.001 \\
\hline c. $2356-63 A>G$ & $21 / 41$ & - & - & - & - & - & - & - & - & - & - & - & - & - & - & - & - & - & - \\
\hline c. $2545+26 \mathrm{G}>\mathrm{A}$ & $22 / 41$ & rs45517242 & 0.000 & 0.000 & 0.000 & 0.000 & 0.002 & 0.000 & 0.002 & 0.005 & 0.003 & 0.001 & 0.002 & 0.001 & 0.000 & 0.004 & 0.004 & 0.005 & 0.000 \\
\hline c. $2545+31 \mathrm{C}>\mathrm{T}$ & $22 / 41$ & rs45517243 & 0.005 & 0.014 & 0.007 & 0.000 & 0.000 & 0.000 & 0.015 & 0.000 & 0.001 & 0.017 & 0.001 & 0.000 & 0.001 & 0.000 & 0.000 & 0.000 & 0.000 \\
\hline c. $2545+95$ dupG & $22 / 41$ & rs397515216 & 0.063 & - & - & - & - & - & - & - & - & - & - & - & - & - & - & - & - \\
\hline c. $2545+135 \mathrm{C}>\mathrm{T}$ & $22 / 41$ & rs139579913 & 0.004 & 0.012 & 0.003 & 0.000 & 0.000 & 0.000 & - & - & - & - & - & - & - & - & - & - & - \\
\hline c. $2545+157 \mathrm{G}>\mathrm{C}$ & $22 / 41$ & rs9941195 & 0.056 & 0.201 & 0.020 & 0.000 & 0.000 & 0.000 & - & - & - & - & - & - & - & - & - & - & - \\
\hline c. $2545+194 A>G$ & $22 / 41$ & rs 7185909 & 0.035 & 0.123 & 0.013 & 0.000 & 0.002 & 0.000 & - & - & - & - & - & - & - & - & - & - & - \\
\hline c. $2545+240 \mathrm{C}>\mathrm{T}$ & $22 / 41$ & rs9921591 & 0.056 & 0.201 & 0.020 & 0.000 & 0.000 & 0.000 & - & - & - & - & - & - & - & - & - & - & - \\
\hline c. $2545+244 C>G$ & $22 / 41$ & rs143553665 & - & 0.012 & 0.001 & 0.000 & 0.000 & 0.000 & - & - & - & - & - & - & - & - & - & - & - \\
\hline c. $2546-12 \mathrm{C}>\mathrm{T}$ & $22 / 41$ & rs 13331451 & 0.206 & 0.620 & 0.107 & 0.000 & 0.085 & 0.052 & 0.521 & 0.074 & 0.095 & 0.552 & 0.063 & 0.074 & 0.000 & 0.098 & 0.072 & 0.083 & 0.048 \\
\hline c. $2639+44 C>G$ & $23 / 41$ & rs 1800715 & 0.215 & 0.624 & 0.115 & 0.000 & 0.097 & 0.074 & 0.526 & 0.087 & 0.107 & 0.557 & 0.069 & 0.102 & 0.000 & 0.111 & 0.085 & 0.099 & 0.063 \\
\hline c. $2639+44 C>T$ & $23 / 41$ & rs 1800715 & - & - & - & - & - & - & - & - & 0.000 & 0.000 & 0.000 & 0.000 & 0.000 & 0.000 & 0.000 & 0.000 & 0.000 \\
\hline c. $2640-74 \mathrm{G}>\mathrm{A}$ & $23 / 41$ & rs143128054 & 0.004 & 0.014 & 0.003 & 0.000 & 0.000 & 0.000 & - & - & - & - & - & - & - & - & - & - & - \\
\hline c. $2640-65 C>T$ & $23 / 41$ & rs138967036 & 0.004 & 0.014 & 0.003 & 0.000 & 0.000 & 0.000 & - & - & - & - & - & - & - & - & - & - & - \\
\hline c. $2640-26 \mathrm{G}>\mathrm{T}$ & $23 / 41$ & rs45483797 & 0.003 & 0.012 & 0.001 & 0.000 & 0.000 & 0.000 & 0.013 & 0.000 & 0.001 & 0.011 & 0.001 & 0.000 & 0.000 & 0.000 & 0.000 & 0.000 & 0.000 \\
\hline c. $2743-40 A>G$ & $24 / 41$ & rs 45517265 & 0.004 & 0.000 & 0.006 & 0.000 & 0.015 & 0.002 & 0.002 & 0.010 & 0.007 & 0.002 & 0.007 & 0.004 & 0.000 & 0.001 & 0.010 & 0.010 & 0.007 \\
\hline c. $2837+93 \mathrm{G}>\mathrm{A}$ & $25 / 41$ & - & - & - & - & - & - & - & - & - & - & - & - & - & - & - & - & - & - \\
\hline c. $2838-4 A>G$ & $25 / 41$ & rs 45517272 & 0.000 & 0.000 & 0.000 & 0.000 & 0.002 & 0.000 & 0.000 & 0.001 & 0.001 & 0.000 & 0.000 & 0.003 & 0.000 & 0.000 & 0.001 & 0.001 & 0.001 \\
\hline c.2966+92_2966+94dup & $26 / 41$ & rs397515128 & - & - & - & - & - & - & - & - & - & - & - & - & - & - & - & - & - \\
\hline c. $2966+217 \mathrm{G}>\mathrm{T}$ & $26 / 41$ & rs118124428 & 0.008 & 0.002 & 0.009 & 0.000 & 0.030 & 0.001 & - & - & - & - & - & - & - & - & - & - & - \\
\hline c. $2967-41 \mathrm{C}>\mathrm{T}$ & $26 / 41$ & rs972958530 & - & - & - & - & - & - & - & - & 0.000 & 0.000 & 0.000 & 0.000 & 0.000 & 0.000 & 0.000 & 0.000 & 0.000 \\
\hline c. $3131+34 \mathrm{C}>\mathrm{T}$ & $27 / 41$ & rs45487103 & 0.000 & 0.000 & 0.000 & 0.001 & 0.000 & 0.000 & 0.000 & 0.001 & 0.001 & 0.000 & 0.001 & 0.002 & 0.000 & 0.000 & 0.001 & 0.001 & 0.000 \\
\hline c. $3132-30 A>G$ & $27 / 41$ & rs45517283 & 0.003 & 0.012 & 0.001 & 0.000 & 0.000 & 0.000 & 0.013 & 0.000 & 0.001 & 0.012 & 0.001 & 0.000 & 0.000 & 0.000 & 0.000 & 0.000 & 0.000 \\
\hline c. $3397+126 C>T$ & $29 / 41$ & rs115243225 & 0.051 & 0.184 & 0.019 & 0.000 & 0.000 & 0.000 & - & - & - & - & - & - & - & - & - & - & - \\
\hline c. $3610+46$ dupC & $30 / 41$ & - & - & - & - & - & - & - & - & - & - & - & - & - & - & - & - & - & - \\
\hline c. $3611-136 \mathrm{C}>\mathrm{T}$ & $30 / 41$ & rs376053649 & - & - & - & - & - & - & - & - & - & - & - & - & - & - & - & - & - \\
\hline c. $3611-100 \mathrm{~T}>\mathrm{C}$ & $30 / 41$ & rs370512781 & - & - & - & - & - & - & - & - & - & - & - & - & - & - & - & - & - \\
\hline c. $3611-28 \mathrm{C}>\mathrm{T}$ & $30 / 41$ & rs45517303 & - & - & - & - & - & - & - & - & 0.000 & 0.000 & 0.000 & 0.000 & 0.000 & 0.000 & 0.000 & 0.000 & 0.000 \\
\hline
\end{tabular}




\begin{tabular}{|c|c|c|c|c|c|c|c|c|c|c|c|c|c|c|c|c|c|c|c|}
\hline c. $3815-124 C>T$ & $31 / 41$ & rs143001593 & 0.003 & 0.009 & 0.001 & 0.000 & 0.001 & 0.000 & - & - & - & - & - & - & - & - & - & - & - \\
\hline c. $3815-38 \mathrm{C}>\mathrm{T}$ & $31 / 41$ & rs371592734 & - & - & - & - & - & - & 0.000 & 0.000 & 0.000 & 0.000 & 0.000 & 0.001 & 0.000 & 0.000 & 0.000 & 0.000 & 0.000 \\
\hline c. $3883+8 \mathrm{C}>\mathrm{G}$ & $32 / 41$ & rs 45517316 & 0.012 & 0.043 & 0.004 & 0.000 & 0.000 & 0.000 & 0.033 & 0.000 & 0.003 & 0.039 & 0.003 & 0.000 & 0.000 & 0.000 & 0.000 & 0.002 & 0.000 \\
\hline c. $3883+66 \mathrm{G}>\mathrm{A}$ & $32 / 41$ & rs74002774 & 0.011 & 0.042 & 0.003 & 0.000 & 0.000 & 0.000 & 0.035 & 0.000 & - & - & - & - & - & - & - & - & - \\
\hline c. $3883+78 \mathrm{G}>\mathrm{A}$ & $32 / 41$ & rs 1800705 & 0.011 & 0.003 & 0.017 & 0.000 & 0.027 & 0.011 & - & - & - & - & - & - & - & - & - & - & - \\
\hline c. $3883+127 \mathrm{G}>\mathrm{A}$ & $32 / 41$ & rs562317274 & 0.001 & 0.004 & 0.000 & 0.000 & 0.000 & 0.000 & - & - & - & - & - & - & - & - & - & - & - \\
\hline c. $3883+226 \mathrm{G}>\mathrm{A}$ & $32 / 41$ & rs9928737 & 0.188 & 0.409 & 0.180 & 0.000 & 0.188 & 0.088 & - & - & - & - & - & - & - & - & - & - & - \\
\hline c. $3884-68 \mathrm{C}>\mathrm{T}$ & $32 / 41$ & rs114209035 & 0.004 & 0.014 & 0.003 & 0.000 & 0.000 & 0.000 & - & - & 0.001 & 0.012 & 0.001 & 0.000 & 0.000 & 0.000 & 0.000 & 0.001 & 0.000 \\
\hline c. $3884-56 \mathrm{C}>\mathrm{G}$ & $32 / 41$ & rs 1800724 & 0.036 & 0.001 & 0.038 & 0.000 & 0.078 & 0.079 & - & - & 0.060 & 0.009 & 0.029 & 0.092 & 0.000 & 0.110 & 0.071 & 0.059 & 0.066 \\
\hline c. $4005+84 \mathrm{C}>\mathrm{T}$ & $33 / 41$ & rs 30259 & 0.040 & 0.002 & 0.053 & 0.046 & 0.074 & 0.041 & - & - & - & - & - & - & - & - & - & - & - \\
\hline c. $4005+153 \mathrm{~T}>\mathrm{A}$ & $33 / 41$ & rs28535326 & 0.019 & 0.070 & 0.006 & 0.000 & 0.000 & 0.000 & - & - & - & - & - & - & - & - & - & - & - \\
\hline c. $4006-60 \mathrm{C}>\mathrm{A}$ & $33 / 41$ & rs376519405 & - & - & - & - & - & - & - & - & - & - & - & - & - & - & - & - & - \\
\hline c. $4006-8 \mathrm{C}>\mathrm{T}$ & $33 / 41$ & rs 45517325 & 0.002 & 0.000 & 0.004 & 0.000 & 0.003 & 0.002 & 0.000 & 0.004 & 0.002 & 0.001 & 0.004 & 0.000 & 0.000 & 0.000 & 0.004 & 0.004 & 0.001 \\
\hline c. $4493+110 \mathrm{G}>\mathrm{A}$ & $34 / 41$ & - & - & - & - & - & - & - & - & - & - & - & - & - & - & - & - & - & - \\
\hline c. $4569+44 C>T$ & $35 / 41$ & rs142227748 & 0.010 & 0.039 & 0.000 & 0.000 & 0.000 & 0.000 & 0.025 & 0.000 & 0.002 & 0.032 & 0.001 & 0.000 & 0.000 & 0.000 & 0.000 & 0.001 & 0.000 \\
\hline c. $4569+46 C>T$ & $35 / 41$ & rs45482793 & 0.004 & 0.001 & 0.003 & 0.000 & 0.018 & 0.001 & 0.003 & 0.018 & 0.010 & 0.002 & 0.005 & 0.010 & 0.000 & 0.008 & 0.017 & 0.011 & 0.003 \\
\hline c. $4569+47 \mathrm{G}>\mathrm{A}$ & $35 / 41$ & rs45517351 & 0.019 & 0.065 & 0.013 & 0.000 & 0.000 & 0.001 & 0.059 & 0.001 & 0.005 & 0.059 & 0.003 & 0.006 & 0.000 & 0.000 & 0.000 & 0.004 & 0.000 \\
\hline c. $4570-38 \mathrm{G}>\mathrm{A}$ & $35 / 41$ & rs770778907 & - & - & - & - & - & - & - & - & 0.000 & 0.000 & 0.000 & 0.000 & 0.000 & 0.000 & 0.000 & 0.000 & 0.000 \\
\hline c. $4662+199 \mathrm{G}>\mathrm{A}$ & $36 / 41$ & rs1379483764 & - & - & - & - & - & - & - & - & - & - & - & - & - & - & - & - & - \\
\hline c. $4663-88 \mathrm{C}>\mathrm{T}$ & $36 / 41$ & rs146465188 & 0.006 & 0.017 & 0.009 & 0.000 & 0.000 & 0.000 & - & - & - & - & - & - & - & - & - & - & - \\
\hline c. $4663-56 C>G$ & $36 / 41$ & - & - & - & - & - & - & - & - & - & - & - & - & - & - & - & - & - & - \\
\hline c. $4849+75 \mathrm{C}>\mathrm{T}$ & $37 / 41$ & rs76029733 & 0.028 & 0.011 & 0.026 & 0.043 & 0.031 & 0.035 & - & - & - & - & - & - & - & - & - & - & - \\
\hline c. $4849+76 \mathrm{G}>\mathrm{A}$ & $37 / 41$ & rs144647393 & 0.015 & 0.057 & 0.001 & 0.000 & 0.001 & 0.000 & - & - & - & - & - & - & - & - & - & - & - \\
\hline c. $4849+97 \mathrm{delA}$ & $37 / 41$ & rs142421783 & 0.056 & 0.201 & 0.020 & 0.001 & 0.000 & 0.000 & - & - & - & - & - & - & - & - & - & - & - \\
\hline c. $4850-133 \mathrm{C}>\mathrm{T}$ & $37 / 41$ & - & - & - & - & - & - & - & - & - & - & - & - & - & - & - & - & - & - \\
\hline c. $4850-109 \mathrm{~T}>\mathrm{C}$ & $37 / 41$ & rs 13335638 & 0.241 & 0.595 & 0.195 & 0.000 & 0.198 & 0.090 & - & - & - & - & - & - & - & - & - & - & - \\
\hline c. $4990-7 \mathrm{C}>\mathrm{T}$ & $38 / 41$ & rs45457095 & 0.009 & 0.027 & 0.012 & 0.000 & 0.000 & 0.000 & 0.028 & 0.001 & 0.002 & 0.025 & 0.002 & 0.006 & 0.000 & 0.000 & 0.000 & 0.002 & 0.001 \\
\hline c. $5160+37 \mathrm{C}>\mathrm{T}$ & $40 / 41$ & rs 45517400 & 0.001 & 0.000 & 0.001 & 0.000 & 0.003 & 0.000 & 0.000 & 0.001 & 0.000 & 0.000 & 0.001 & 0.000 & 0.000 & 0.000 & 0.001 & 0.000 & 0.000 \\
\hline c. $5160+43 C>T$ & $40 / 41$ & rs45517402 & - & - & - & - & - & - & - & - & 0.000 & 0.000 & 0.000 & 0.000 & 0.000 & 0.000 & 0.000 & 0.000 & 0.000 \\
\hline
\end{tabular}




\begin{tabular}{|c|c|c|c|c|c|c|c|c|c|c|c|c|c|c|c|c|c|c|c|}
\hline c.5161-28_5161-25delTGAG & $40 / 41$ & rs1799758 & 0.005 & - & - & - & - & - & - & - & - & - & - & - & - & - & - & - & - \\
\hline c. $5161-10 \mathrm{~A}>\mathrm{C}$ & $40 / 41$ & rs 1800718 & 0.257 & 0.616 & 0.213 & 0.000 & 0.236 & 0.090 & 0.543 & 0.219 & 0.200 & 0.563 & 0.128 & 0.250 & 0.000 & 0.258 & 0.214 & 0.217 & 0.097 \\
\hline c. $5260-49 \mathrm{C}>\mathrm{T}$ & $41 / 41$ & rs 13332221 & 0.168 & 0.458 & 0.140 & 0.000 & 0.116 & 0.020 & 0.397 & 0.102 & 0.106 & 0.410 & 0.083 & 0.142 & 0.000 & 0.114 & 0.103 & 0.125 & 0.028 \\
\hline c. $5260-25 C>G$ & $41 / 41$ & rs13332222 & 0.201 & 0.576 & 0.153 & 0.000 & 0.118 & 0.020 & 0.478 & 0.105 & 0.114 & 0.507 & 0.089 & 0.142 & 0.000 & 0.115 & 0.106 & 0.130 & 0.029 \\
\hline c. $5260-15 \mathrm{C}>\mathrm{T}$ & $41 / 41$ & rs 45517416 & 0.002 & 0.004 & 0.000 & 0.000 & 0.001 & 0.002 & 0.002 & 0.001 & 0.002 & 0.002 & 0.001 & 0.000 & 0.000 & 0.001 & 0.002 & 0.002 & 0.002 \\
\hline
\end{tabular}


IX. Supplementary Figures
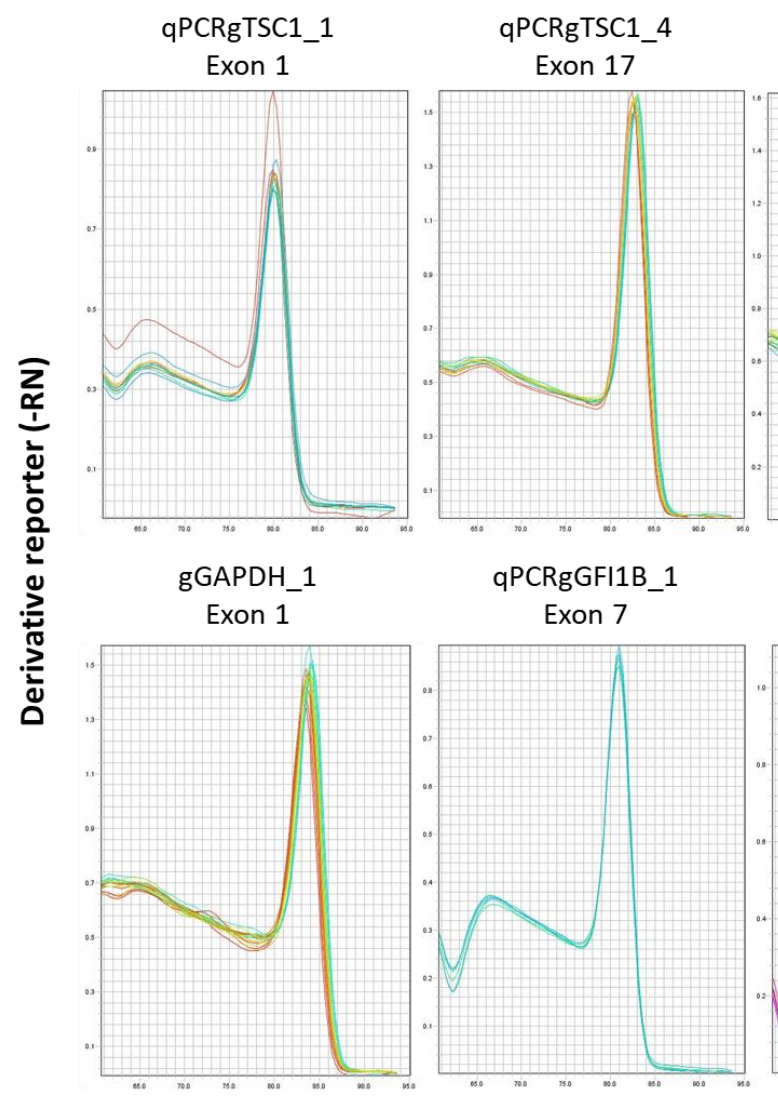

qPCRgTSC2_7

Exon 9

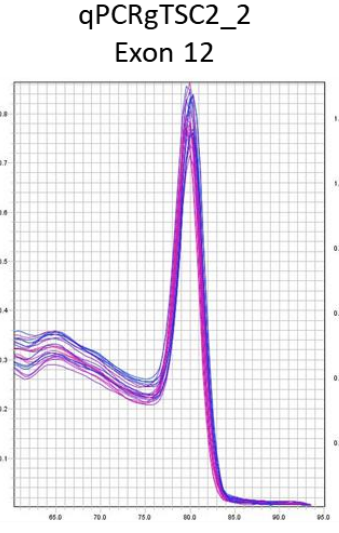

qPCRgTSC2_4

Exon 19

qPCRgTSC2_8

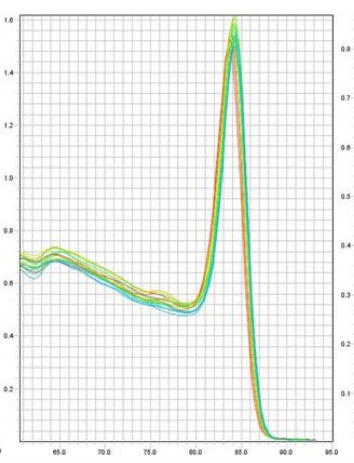

CRgGFI1B_

qPCRgSPACA9_2

qPCRgNTHL1_

Exon 4

Exon 6

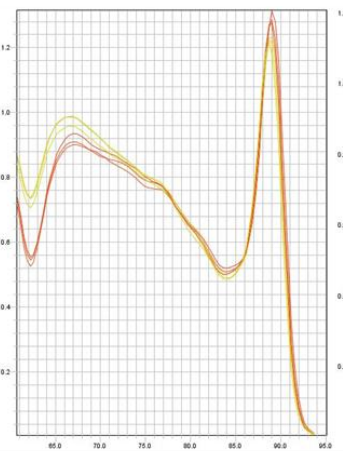

qPCRgPKD1_1

Exon 45
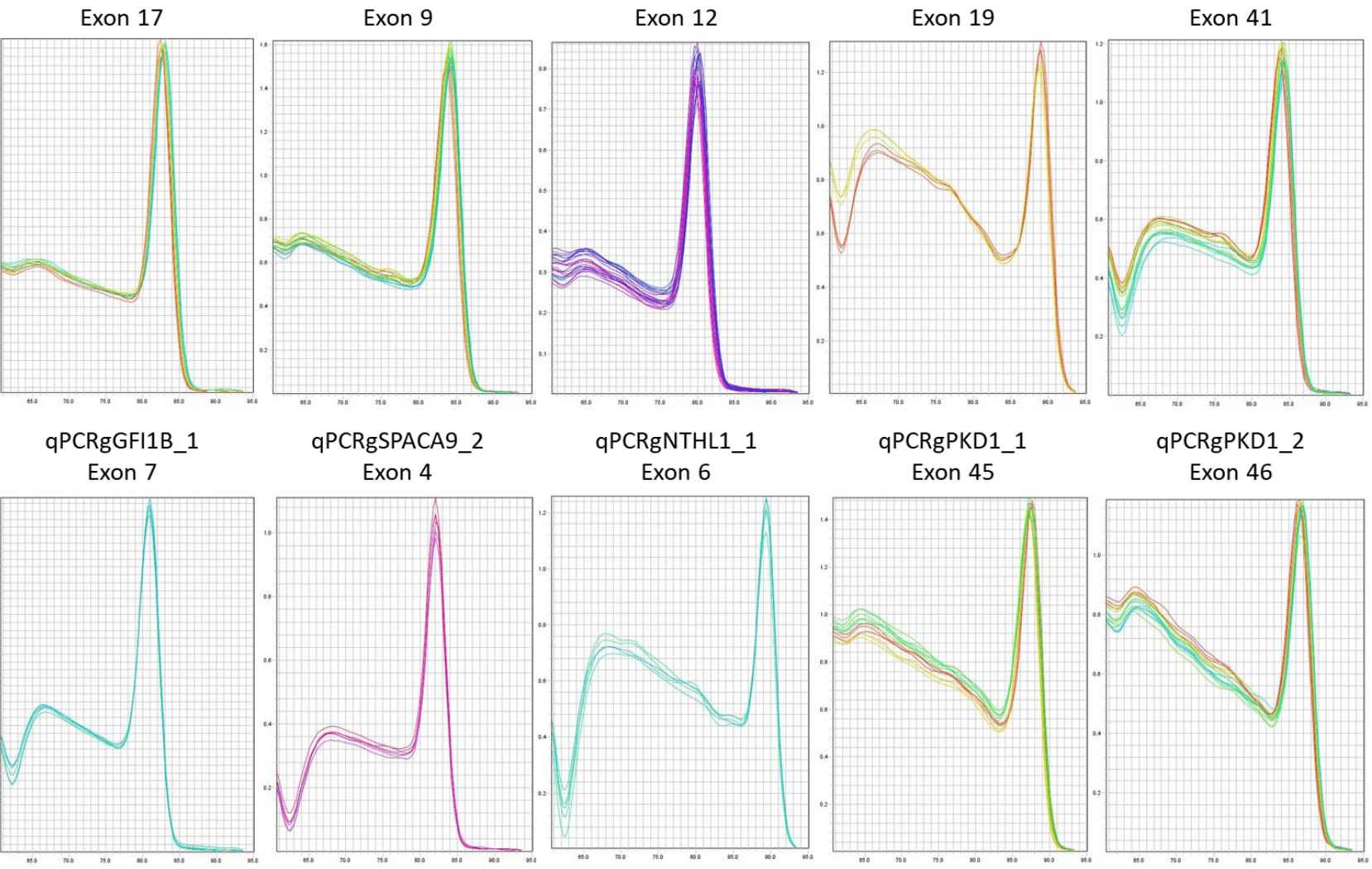

qPCRgPKD1_2

Exon 46

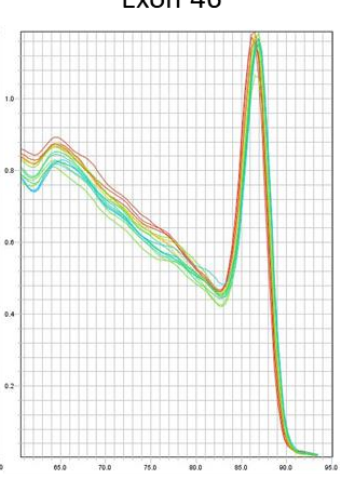

Temperature $\left({ }^{\circ} \mathrm{C}\right)$

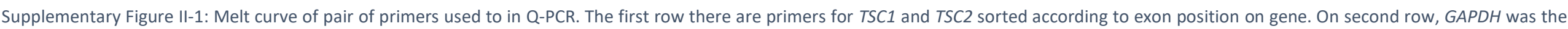

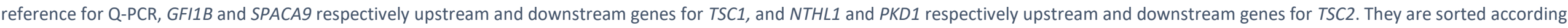
to exon position on gene 


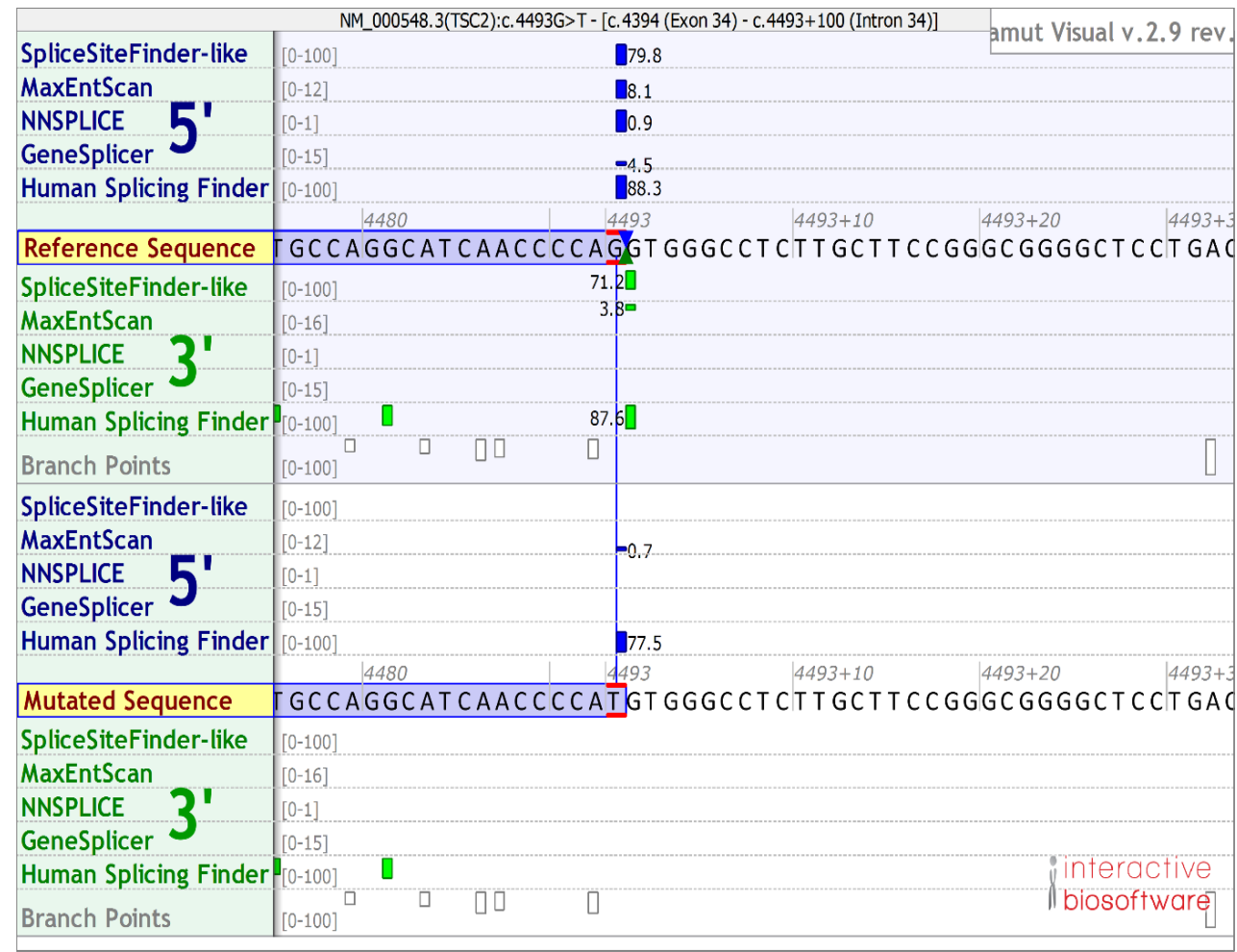

Supplementary Figure II-2: ALAMUT splicing site prediction analysis for TSC2 (NM_000548.3) c.4493G>T substitution. 


\section{Chapter III}

\section{Assessment of regulatory relationships between TSC1 and TSC2 mRNA and protein}




\section{List of Acronyms}

3'-UTR: 3'- untranslated region

5'-UTR: 5'-untranslated regions

DMEMplus: DMEM supplemented with $5 \%$ calf serum and penicillin-streptomycin

DMEMminus: DMEM with no supplementation

DPBS: Dulbecco's Phosphate Buffered Saline

DMEM: Dulbecco's Modified Eagle's Medium

EDTA: Ethylenediamine tetra-acetic acid

FDR: False discovery rate

GO: Gene ontology

HSP90: Heat shock protein 90-alpha

O/N: Overnight

TPM: Transcript per million

UTR: Untranslated region 


\section{List of Tables}

Table III-1: Total of samples per cell line for each treatment - DMEMplus or DMEMminus. 134

Table III-2: Observed TSC1 and TSC2 mRNA and protein levels for each knock-out cell line comparatively to HEK293T. 143

Table III-3: Number of genes down- or up-regulated in 1C2 cell line in regard to HEK293T in DMEMplus or DMEMminus groups. 143

Table III-4: Gene sets significantly enriched in down-regulated genes in 1C2 cell line in regard to HEK293T, in DMEMminus group. 144

Table III-5: Gene sets significantly enriched in down-regulated genes in 1C2 cell line in regard to HEK293T, in DMEMplus group. 144

Table III-6 Gene sets significantly enriched in up-regulated genes in 1C2 cell line in regard to HEK293T, in DMEMminus group. 144

Table III-7: Gene sets significantly enriched in up-regulated genes in 1C2 cell line in regard to HEK293T, in DMEMplus group. . .145

Table III-8: Number of genes observed when groups assigned in Table 13 were intersected. 145

Table III-9: Gene sets significantly enriched in down-regulated genes in 1C2 cell line in regard to HEK293T, independently on the serum presence. 146

Table III-10: Gene sets significantly enriched in down-regulated genes in 1C2 cell line in regard to HEK293T, detected in DMEMplus but not in DMEMminus samples. 146

Table III-11: Gene sets significantly enriched in up-regulated genes in 1C2 cell line in regard to HEK293T, detected in DMEMminus but not in DMEMplus samples.

Table III-12: Gene sets significantly enriched in up-regulated genes in 1C2 cell line in regard to HEK293T, detected in DMEMplus but not in DMEMminus samples....

Table III-13: Gene sets significantly enriched in down-regulated genes in DMEMminus group in regard to DMEMplus, in the $1 C 2$ cell line. 148

Table III-14: Gene sets significantly enriched in up-regulated genes in DMEMminus group in regard to DMEMplus, in the $1 C 2$ cell line. 148 


\section{List of Figures}

Figure III-1: TSC1 locus and mRNA expression. 126

Figure III-2: TSC2 locus and mRNA expression. 127

Figure III-3: Cladogram and genotype of wildtype cell line and six edited cell lines by the CRISPR-Cas9.......132

Figure III-4: Workflow of cell culture, DNA isolation, RNA Isolation and protein isolation from all seven cell lines. O/N: overnight. 133

Figure III-5: TSC1, TSC2, AKT and S6 protein levels assessed by Western blotting. .137

Figure III-6: Specific protein profile from genetically modified cell lines.... 139

Figure III-7: TSC1 and TSC2 mRNA RPKM upon DMEMplus and DMEMminus treatments 140

Figure III-8: Log2FoldChange of TSC1 (A) and TSC2 (B) transcript amount upon DMEMplus treatment. Asterisk $=p$-value $<0.05$. 141

Figure III-9: Log2FoldChange of TSC1 (A) and TSC2 (B) transcript amount upon DMEMminus treatment. Asterisk $=p$-value $<0.05$. 


\section{Summary}

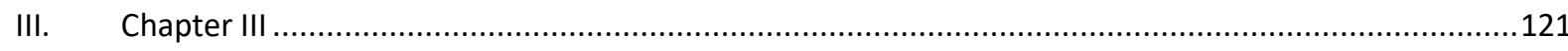

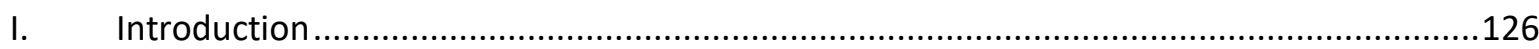

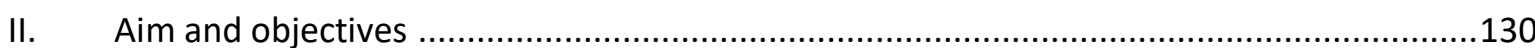

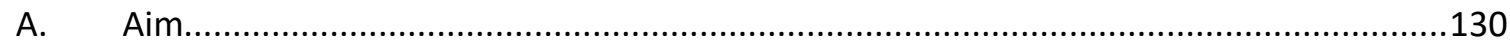

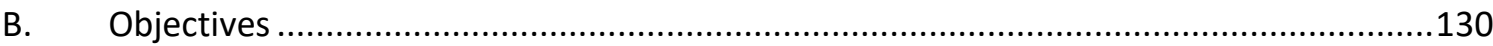

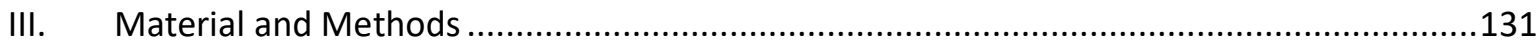

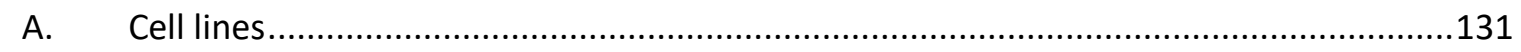

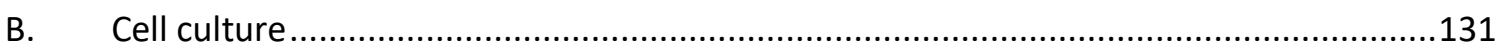

C. Protein isolation and Western Blotting .......................................................... 133

D. Total RNA isolation and RNA sequencing ...................................................... 133

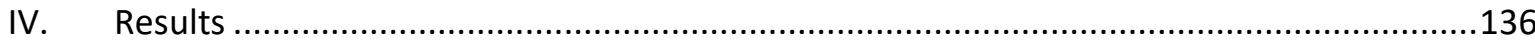

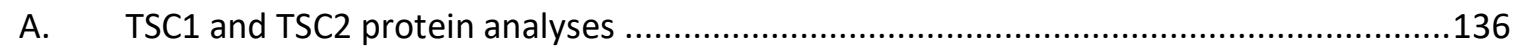

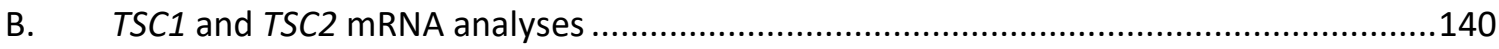

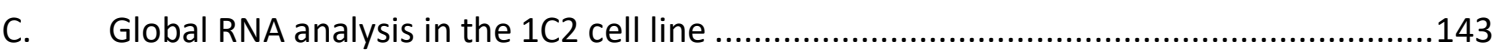

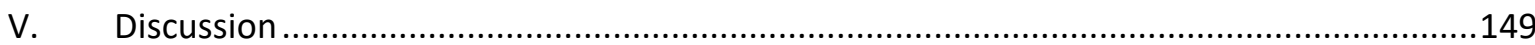

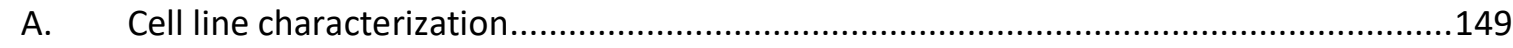

B. TSC1 and TSC2 gene expression regulation.................................................. 151

C. Protein stress response in TSC1-negative cell line ............................................152

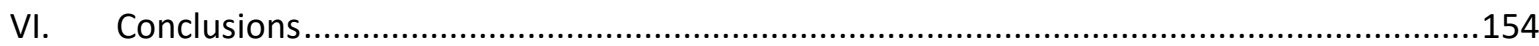

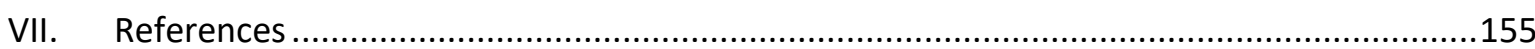

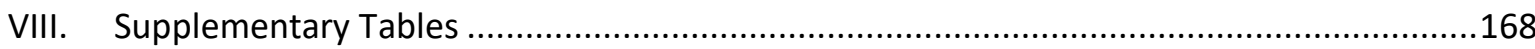

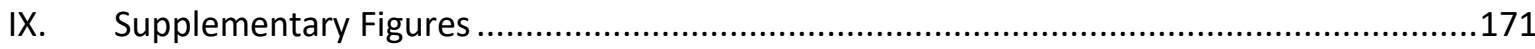




\section{Introduction}

Human TSC1 (NG_012386.1) exons 1, 2 and the first 80 bp of exon 3 comprise the $5^{\prime}$ untranslated region (UTR) of its mRNA. TSC1 3'-UTR corresponds to the last 4,887 bp of exon 23 (Figure III-1A). There are $68 \%$ identity between human and mouse TSC1 3'-UTR, suggesting it lays as a platform for potential binding sites for translation and stability regulatory factors. Full-length TSC1 mature mRNA (NM_000368.4) consists of 8,626 nucleotides, and is expressed in nearly all tissues. RNA-Seq expression data from the Genotype-Tissue Expression (GTEx) project reveals TSC1 highest median expression of transcript per million (TPM) in the cerebellum (Figure III-1B).

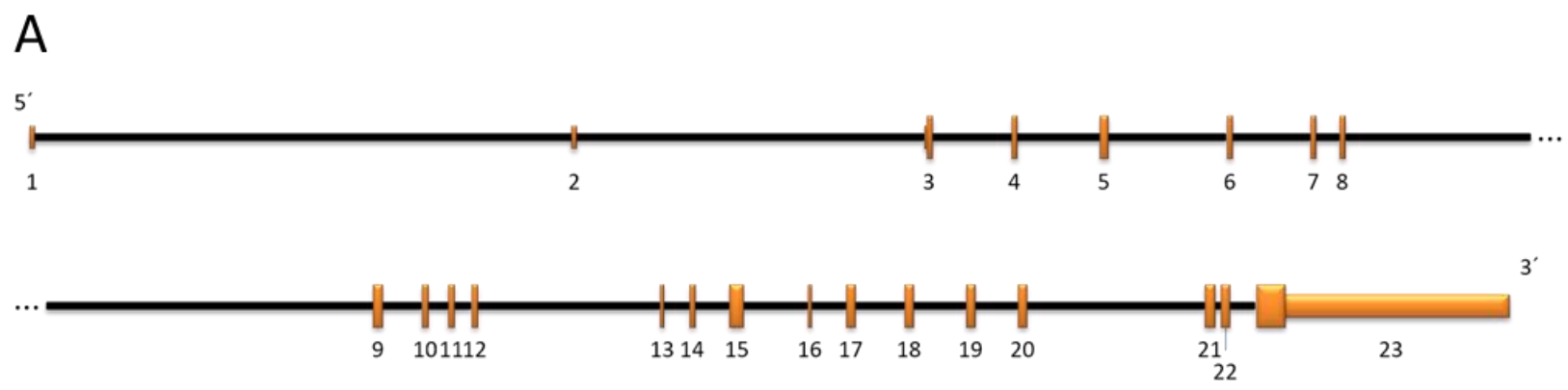

B

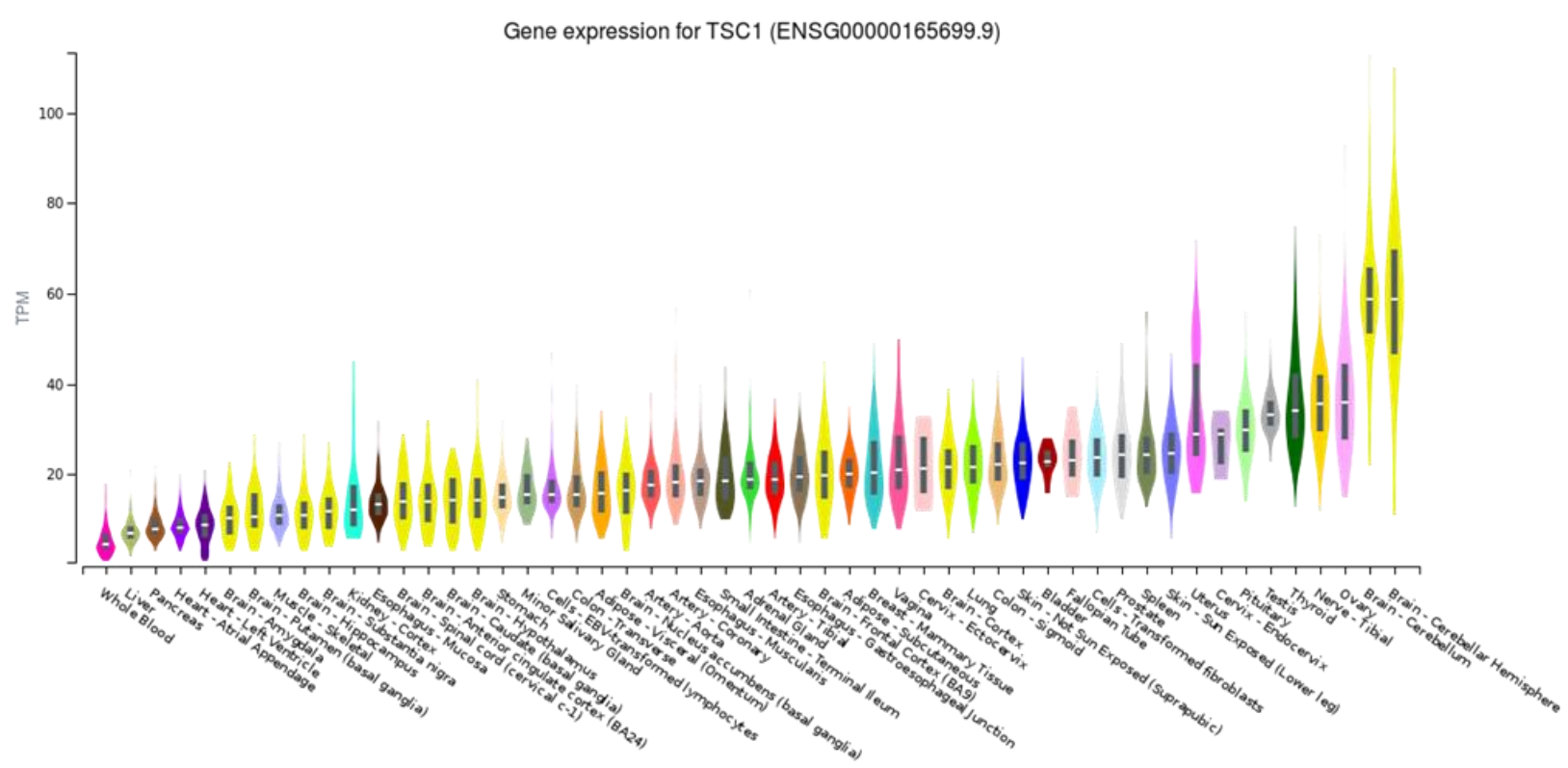

Figure III-1: TSC1 locus and mRNA expression. (A) Schematic diagram of the TSC1 gene. Black lines represent introns, broad and narrow orange bars highlight respectively coding exons and the UTR regions of the gene. (B) Plot of TSC1 RNA-Seq expression data from the GTEx project (https://www.gtexportal.org/home/gene/TSC1). TPM: transcripts per million.

TSC2 (NG_005895.1) exon 1 and the first 29 bp of exon 2 encompass the 5 '-UTR of its mRNA. The 3'UTR comprises the last 102 bp of exon 42. The full-length TSC2 transcript (NM_000548.3) consists of 5,675 
nucleotides and, similar to TSC1, is expressed in most tissues, with the highest mRNA amount in the cerebellum (Figure III-2B). Overall TSC2 mRNA levels are nearly twice that observed for TSC1 (Figure III-1B and Figure III-2B).
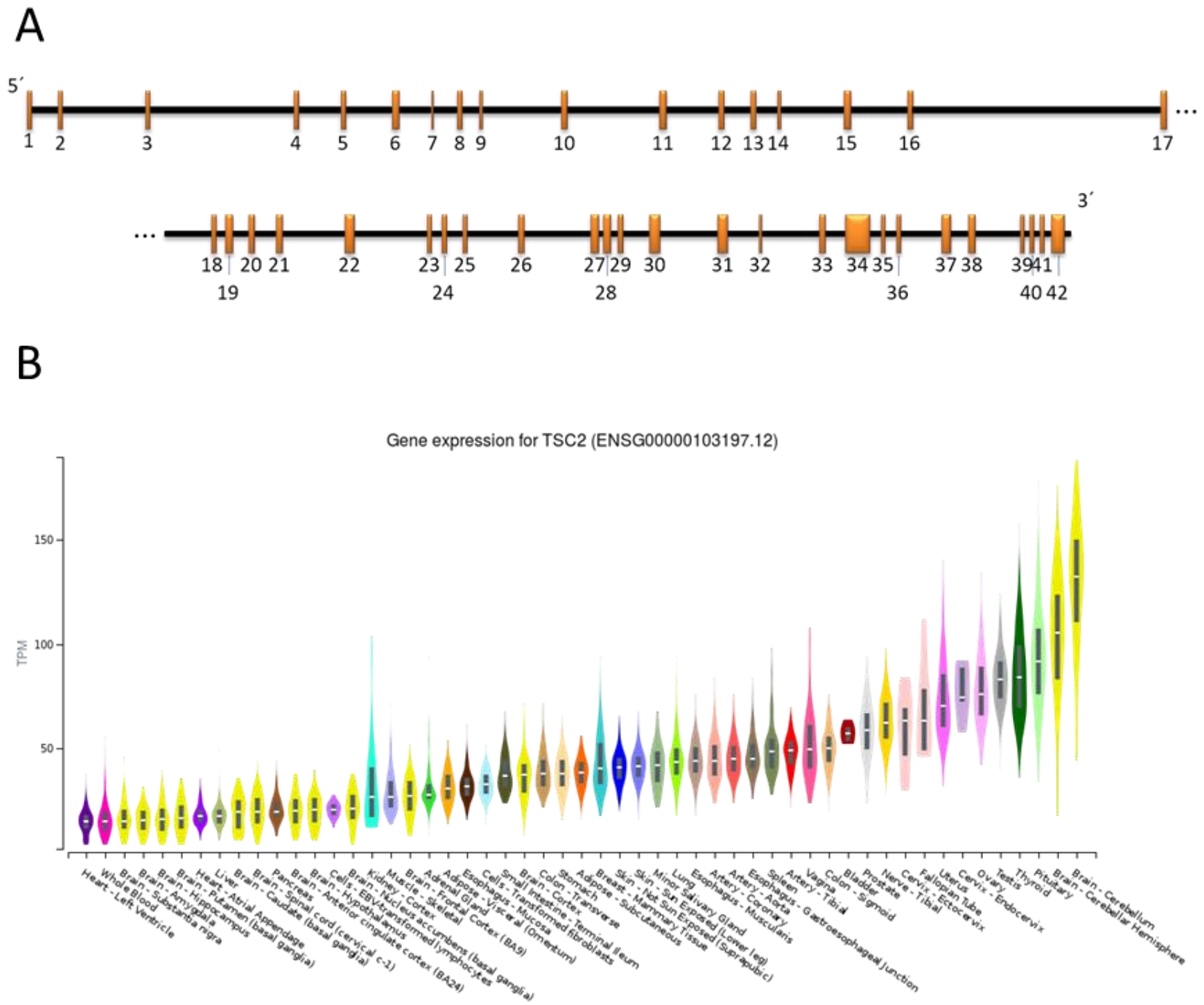

Figure III-2: TSC2 locus and mRNA expression. (A) Schematic diagram of the TSC2 gene exons and introns. Black lines represent introns, broad and narrow orange bars highlight coding exons and the UTR regions, respectively. (B) TSC2 RNA-Seq expression data from the GTEx project (https://www.gtexportal.org/home/gene/TSC2). TPM: transcripts per million.

TSC1 protein (NP_000359.1) is a 130-kDa (1,164 amino acids) hydrophilic protein displaying a Cterminal domain required for interaction with TSC2 (residues 762-848) (van Slegtenhorst, Nellist et al. 1998), predicted as a coiled-coil region. The 200 kDa TSC2 protein (NP_000539.2) has 1,807 amino acids, an Nterminal domain (residues 555-903) required for interaction with TSC1, and a C-terminal 163-residue GTPase activating (GAP) domain (GAP-Rheb residues 1562 to 1764) (Plank, Yeung et al. 1998, van Slegtenhorst, Nellist et al. 1998). Under conditions of amino acid depletion, Rag GTPase recruits TSC2 to the lysosome, interacting with Rheb. mTORC1 is thus released from the lysosome and becomes inactivated. However, upon amino acid 
withdrawal, cells with pathogenic variants on TSC2 cannot completely release TORC1 from the lysosome, hence failing to adjust to physiological conditions (Demetriades, Doumpas et al. 2014).

In cells lacking the TSC2 protein, TSC1 homodimers are prone to self-aggregation in detergentresistant complexes, presenting a coarsely dotted cytoplasmic distribution by immunofluorescence analysis (Nellist, Sancak et al. 2005). If both genes are co-overexpressed, TSC1 protein becomes solubilized with a more homogeneous cytoplasmic distribution. Thus, it has been proposed that TSC2 may act as TSC1 chaperone (Nellist, van Slegtenhorst et al. 1999). On the other hand, Benvenuto, Li et al. (2000) suggested that TSC1 stabilizes TSC2.

TBC1D7, which has been reported as a protein from the TSC1/2 complex, stabilizes TSC1 dimers (Gai, Chu et al. 2016). The crystal structure of TBC1D7 in complex with the C-terminal part of TSC1 (TSC1-CC, residues 939-992, encoded by exon 22) revealed that two units of TSC1-CC form a parallel homodimer - two symmetric surfaces for interaction with TBC1D7 (Santiago Lima, Hoogeveen-Westerveld et al. 2014, Gai, Chu et al. 2016, Qin, Wang et al. 2016), stabilizing the TSC complex. Furthermore, the molecular chaperone heatshock protein 90-alpha (HSP90, NP_001017963.2), an essential component of the cellular homeostatic machinery in eukaryotes, may interact with TSC1 in the absence or presence of TSC2. The association between TSC1 and TSC2 decreases under heat shock, which does not affect the association between TSC1 and HSP90 (Inoue, Uyama et al. 2010). TSC1 has been proposed as HSP90 co-chaperone (Woodford, 2017; Sager 2018a, 2018b), as HSP90 binds in vitro and in vivo to denatured proteins and displays ATP-dependent anti-aggregation properties (Panaretou, Prodromou et al. 1998).

TSC2 had been identified as a target of AKT kinase regulation (Potter, Huang et al. 2001, Dan, Sun et al. 2002, Manning, Tee et al. 2002, Inoki, Li et al. 2003). The pathway of phosphatidylinositol-3 and AKT kinases was characterized as the main physiological regulation of TSC complex turnover, leading to a decrease in the affinity between TSC1 and TSC2, and degradation of both proteins mediated by their phosphorylation (Potter, Huang et al. 2001, Dan, Sun et al. 2002, Inoki, Li et al. 2003). The observed response to heat shock appears to be related to the phosphorylation of TSC2 by AKT, stimulating its degradation by the proteasome (Inoue, Uyama et al. 2010). The interaction of TSC1 with HSP9O is required for its localization in the outer membrane of the mitochondria, with or without TSC2, in a manner dependent on the phosphorylation of TSC1-Thr ${ }^{417}$ (Inoue, Uyama et al. 2010). These data are corroborated by other observations that the knockout of Tsc2 maintains Tsc1 at reasonable levels (Zhang, Gao et al. 2003, Pollizzi, Malinowska-Kolodziej et al. 2009). On the other hand, Western blotting of mouse $\mathrm{TsC1}^{\%}$ embryonic fibroblast protein has shown reduced levels of the Tsc2 protein (Astrinidis, Senapedis et al. 2006), suggesting that lack of expression of both Tsc1 alleles may subject Tsc2 to poly-ubiquitination and degradation by the proteasome. Overexpression of TSC1 in Rat-2 cells or rat embryonic fibroblasts elevates the level of TSC2, and binding of TSC1 to TSC2 inhibits TSC2 ubiquitination. Finally, inhibition of the proteasome represses TSC2 degradation (Benvenuto, Li et al. 2000). 
The stability of TSC1-TSC2 protein complex is central for the control of mTORC1 signaling. Further understanding of the complexity of TSC1 and TSC2 gene regulation is important as heterologous gene replacement can be a potential future therapy alternative for specific TSC lesions (Prabhakar, Zhang et al. 2015). However, it is as yet unknown whether in the absence of the TSC1 or TSC2 protein there may be transcriptional or post-transcriptional feedback control of TSC1 and TSC2 gene expression. Although it is clear that there should be inter-regulation of TSC1 and TSC2 protein stability, it is necessary to specifically understand the levels and mechanisms of regulation of endogenous gene expression. Hence, changes in mRNA amount may build a feedback control loop on TSC gene transcription, e. g., absence of TSC1 gene expression may potentially affect the TSC2 mRNA or TSC2 protein. On the other hand, similar amounts of TSC1 mRNA would refute the hypothesis of transcriptional or mRNA half-life regulation. Moreover, the absence of TSC1 should compromise the cell homeostasis under amino acid withdrawal due to lack of interaction with TSC2 or Hsp90. In this study, we intend to start understanding the regulatory relationship between TSC1 and TSC2 mRNA and protein. 


\section{Aim and objectives}

\section{A. Aim}

To assess TSC1 and TSC2 gene product levels in search for possible transcriptional or translational feedback regulatory loops in cells lacking TSC1 or TSC2 proteins, and transcriptome response of TSC1 knockout cell line on deprivation environment.

\section{B. Objectives}

1. To compare TSC1 and TSC2 protein levels between the reference human cell line and human cell lines with bi-allelic inactivation of TSCl and/or TSC2 genes;

2. To assess the activity of the mTORC1 pathway reported by the phosphorylation of S6 protein $\operatorname{Ser}^{235}$ and $\mathrm{Ser}^{236}$ residues; having nutrient starvation, insulin treatment and rapamycin supplementation as proof-ofconcept controls;

3. To compare the transcriptome between cells with bi-allelic inactivation of TSCl the reference human cell; and

4. To compare the transcriptome of cells with bi-allelic inactivation of $\mathrm{TSCl}$ growing under complete medium and without supplementation. 


\section{Material and Methods}

Experiments were conducted in the Netherlands, mostly at Erasmus Medical Centre, Rotterdam, Amsterdam University or at the GenomeScan (Leiden, the Netherland). Data analyses was performed at Instituto de Biociências, Universidade de São Paulo (São Paulo, Brazil) and Hospital Sírio-Libanês, São Paulo, Brazil.

\section{A. Cell lines}

Seven cell lines employed had been generated before: HEK293T cell line, and six cell lines derived from HEK293T in which TSC1 or TSC2 gene genomic segments have been genetically edited by the CRISPR-Cas9 strategy (Dufner-Almeida et al., submitted). Figure III-3 depicts the outflow of genetic modifications, and Table III-1 presents cell line names and TSC1 and TSC2 genotypes.

\section{B. Cell culture}

Cell lines were seeded in round tissue culture dish (6 cm; Sigma-Aldrich, St. Louis, Missouri, USA) in Dulbecco's Modified Eagle's Medium (DMEM, BioWhittaker, Radnor, Pennsylvania, USA), supplemented with $5 \%$ fetal calf serum and penicillin-streptomycin, constituting the complete medium or DMEMplus, and maintained at $37^{\circ} \mathrm{C}$ and $10 \% \mathrm{CO}_{2}$. After splitting to ten round dishes, cells were kept under the same culturing conditions until $80 \%$ confluence. Cells from three plates (group DMEMplus) were harvested and submitted to DNA, RNA and protein isolation. After washing the cells three times in PBS (Dulbecco's Phosphate Buffer Saline $(0.0095 \mathrm{M})$ - BioWhittaker - Radnor, Pennsylvania, USA), medium from the seven remaining plates was switched to DMEM minimum medium (without supplementation), considered as DMEMminus. The starvation period lasted for 16 hours (ON - overnight), after which RNA and protein were isolated from two plates (group DMEMminus). Rapamycin (Cell Signaling Technology, Danvers, Massachusetts, USA) suspended in DMSO was added to cells from the five remainder dishes to final concentration of $10 \mathrm{nM}$. After one hour, RNA and protein were isolated from two plates (group DMEMminus - RAPA-1h) while culture continued for further 15 hours in three plates. On the fourth day, RNA and protein were isolated from two plates (DMEMminus - RAPA O/N) and cells in the final plate were treated with insulin at $200 \mathrm{nM}$ suspended in HEPES buffer (Sigma-Aldrich, St. Louis, Missouri, USA) for 10 minutes (group DMEMminus - RAPA O/N - INS), washed and harvested for protein isolation (Figure II-2). All seven cell lines were simultaneously submitted to the same protocol, and the experiment was repeated at least three times. 
A

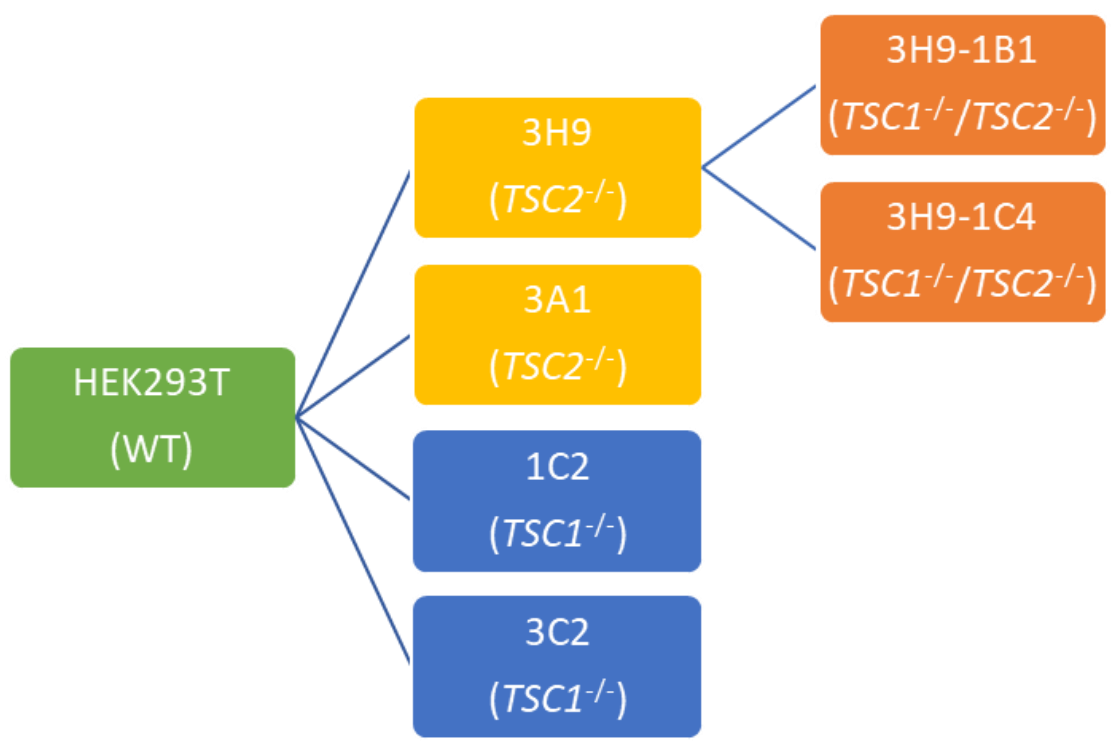

B

\begin{tabular}{|c|c|c|}
\hline Cell Line & TSC1 genotype & TSC2 genotype \\
\hline HEK293T (WT) & c.965T>C (p.M322T) heterozygote & wild-type \\
\hline $\begin{array}{l}1 \mathrm{C} 2 \\
\left(\mathrm{TSC}^{-\%}\right)\end{array}$ & $\begin{array}{l}\text { c.-13_5del homozygote } \\
\text { c.965T>C (p.M322T) heterozygote } \\
\text { c.3194_3206del (p.T1065Kfs*22) homozygote }\end{array}$ & wild-type \\
\hline $\begin{array}{l}3 \mathrm{C} 2 \\
(\operatorname{TSC} 1 \%)\end{array}$ & $\begin{array}{l}\text { c.989_3199del (p.S331_M1067del) heterozygote } \\
\text { c.1002_3204del (p.T335Kfs*22) heterozygote } \\
\text { c.1002dup (p.T335Dfs*6) homozygote } \\
\text { c.3196insT (p.T1066lfs*33) homozygote }\end{array}$ & wild-type \\
\hline $\begin{array}{l}3 \mathrm{~A} 1 \\
\left(\mathrm{TSC} 2^{-\%}\right)\end{array}$ & c.965T>C (p.M322T) heterozygote & $\begin{array}{l}\text { c.121dup (p.T41Nfs*26) homozygote } \\
\text { c.116_4989+358del }\left(p .139 R^{*}{ }^{*} 42\right) \text { heterozygote }\end{array}$ \\
\hline $\begin{array}{l}3 \mathrm{H} 9 \\
\left(\mathrm{TSC2}^{-/}\right)\end{array}$ & c.965T>C (p.M322T) heterozygote & c.122_4990-284del38842 (p.T41Rfs*42) homozygote \\
\hline 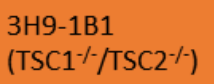 & c.-8_3196del (p.?) homozygote & c.122_4990-284del38842 (p.T41Rfs*42) homozygote \\
\hline $\begin{array}{l}3 \mathrm{H} 9-1 \mathrm{C} 4 \\
\left(\mathrm{TSC}^{-1-} / \mathrm{TSC}^{-1} \text { ) }\right.\end{array}$ & c.-8_3196del (p.?) homozygote & c.122_4990-284del38842 (p.T41Rfs*42) homozygote \\
\hline
\end{tabular}

Figure III-3: Cladogram and genotype of wildtype cell line and six edited cell lines by the CRISPR-Cas9. (A) Schematic representation of parental wild type HEK293T cell line and derived cell line. (B) Genotype of each seven cell lines. 


\section{Protein isolation and Western Blotting}

Cells were lysed in $50 \mathrm{mM}$ Tris- $\mathrm{HCl}(\mathrm{pH} 7.6), 100 \mathrm{mM} \mathrm{NaCl}, 50 \mathrm{mM} \mathrm{NaF}, 1 \%$ Triton-X-100, $1 \mathrm{mM}$ ethylenediamine tetra-acetic acid (EDTA) and complete protease inhibitor cocktail (Roche, Basileia, Switzerland). Proteins lysate were submitted to Criterion ${ }^{\text {TM }}$ TGX ${ }^{\mathrm{TM}}$ Precast Gel (4 -15\%; Bio-Rad, Hercules, California, USA) electrophoresis at 100V for 10-15 minutes followed by one hour at 120V; and transferred to nitrocellulose filters in the Trans-blot ${ }^{\circledR}$ Turbo $^{\mathrm{TM}}$ (Bio-Rad, Hercules, California, USA) using the pre-set program for high molecular weight (2.5 Amp, $25 \mathrm{~V}, 10 \mathrm{~min}$.). Finally, the membrane proteins were blocked in $5 \%$ powder milk in DPBS (Dulbecco's Phosphate Buffer Saline (0.0095M) - BioWhittaker - Radnor, Pennsylvania, USA) for 30 minutes, followed by specific primary and secondary antibody incubations, and fluorescence captured under the conditions described in chapter 2. The experiment was repeated at least three times and Student's $t$ test was used for pairwise comparison.

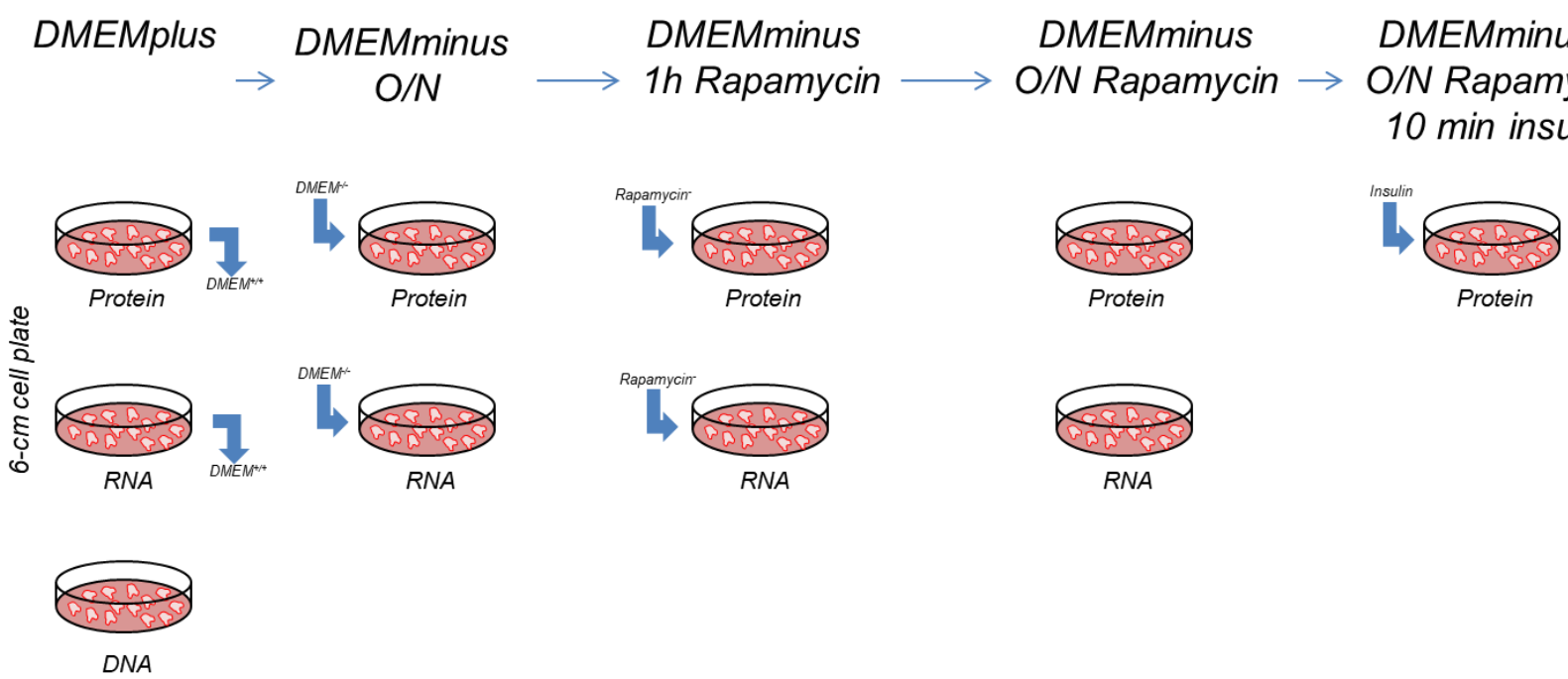

Figure III-4: Workflow of cell culture, DNA isolation, RNA Isolation and protein isolation from all seven cell lines. O/N: overnight.

\section{Total RNA isolation and RNA sequencing}

Total RNA was isolated with RNeasy ${ }^{\circledR}$ Mini Kit (QIAGEN, Hilden, Germany) according to the manufacturer conditions. Briefly, cells were washed in DPBS, and then lysis buffer - $1 \% \beta$-mercaptoethanol in RTL buffer (QIAGEN, Hilden, Germany) - was added. Cells were detached and transferred to a 1.5-mL tube. One volume of $70 \%$ ethanol was added, homogenized, and the solution was transferred to an RNeasy ${ }^{\circledR}$ minispin column, and centrifuged for 15 seconds at $8,000 \times \mathrm{g}$. Column was washed with buffer RW1 and centrifuge for $15 \mathrm{sec}$ at 12000g., and contents incubated with DNase I for 15 minutes at room temperature. Finally, the column was washed three times and RNA eluted in $50 \mu \mathrm{L}$ of RNase-free water. RNA concentration and quality 
were measured using the NanoDrop spectrophotometer (Thermo Fisher Scientific, Waltham, Massachusetts, USA) and BioAnalyzer (Thermo Fisher Scientific, Waltham, Massachusetts, USA) ().

A total of 30 RNA samples (Table III-1) were used for library construction and Illumina HiSeq 4000 sequencing at GenomeScan (Leiden, The Netherlands). To assess the quality of the samples, concentration was determined using the Fragment Analyzer. Sample preparation was performed according to NEBNext Ultra Directional RNA Library Prep Kit for Illumina protocol (NEB \#E7420S/L; New England Biolabs, Ipswich, Massachusetts, USA). Briefly, mRNA was isolated from total RNA using oligo-dT magnetic beads. After mRNA fragmentation on broad peak between 300-500 bp, cDNA was synthesized following kit specifications. Ligation with sequencing adapters followed, and the resulting product was submitted to PCR bridge amplification. Product quality and yield after sample preparation was measured with the Fragment Analyzer (Supplementary Figure III-2). The size of the resulting products was consistent with the expected size distribution (a broad peak between 300-500 nucleotides). A cDNA concentration of 3.0 nM was used (Supplementary Table III-2). Clustering and cDNA sequencing using the Illumina cBot and HiSeq 4000 were performed according to the manufacturer's protocols (Supplementary Table III-3).

Table III-1: Total of samples per cell line for each treatment - DMEMplus or DMEMminus.

\begin{tabular}{lcc}
\hline \multirow{2}{*}{ Cell Line } & \multicolumn{2}{c}{ Treatment } \\
& DMEMplus & DMEMminus \\
\hline HEK293T (WT) & 3 & 3 \\
1C2 $\left(\mathrm{TSC}^{-/-}\right)$ & 2 & 2 \\
$3 \mathrm{C} 2\left(\mathrm{TSC1}^{-/-}\right)$ & 2 & 2 \\
$3 \mathrm{~A} 1\left(\mathrm{TSC}^{-/-}\right)$ & 2 & 2 \\
$3 \mathrm{H} 9\left(\mathrm{TSC}^{-/-}\right)$ & 2 & 2 \\
$3 \mathrm{H} 9-1 \mathrm{~B} 1\left(\mathrm{TSC1}^{-/-} / \mathrm{TSC}^{-/-}\right)$ & 2 & 2 \\
$3 \mathrm{H} 9-1 \mathrm{C} 4\left(\mathrm{TSC1}^{-/-} / \mathrm{TSC}^{-/-}\right)$ & 2 & 2 \\
\hline
\end{tabular}

The mRNA-Seq pipeline starts with quality filtering and trimming of the sequence of optimized standard thresholds: Illumina sequencing adapters were removed, bases with read Q-score below 22 trimmed off, base reads with at least 36 nucleotide-long kept in the data set. Alignment was based on Burrows-Wheeler Transform to ensure maximum sensitivity for mapping reads to exons of genes. All the genes found in the reference annotation were included, even if they do not have any associated expression. Each expressed gene is listed separately per Ensembl Gene ID.

Statistical analysis and comparison between treatment and between samples were performed using DEseq2 script (Love, Huber et al. 2014) on R program (Version 3.5.1, ). Briefly, average of the normalized count values divided by size factors, taken over all samples (baseMean); expression changed due to treatment or cell lines (log2FoldChange), standard error estimate for the log2 fold change (IfCSE), pvalue and padj (BenjaminiHochberg $\mathrm{BH}$-adjusted pvalues for false positive results) were obtained. All pvalues in results' table assigned 
as NA were excluded from analysis because it contained an extreme count outlier, or the expression was not detected. Only genes with padj<0.1 were considered differentially expressed.

Comparisons between gene sets identified from RNA-Seq analysis between 1C2 and HEK293T cell lines from DMEMplus or DMEMminus groups or from 1C2 (DMEMplus) and 1C2 (DMEMminus) groups yielded differentially expressed genes under significance of adjusted $p$ value $<0.1$. Differentially up- or down-regulated genes were submitted to gene ontology (GO) grouping for non-redundant biological processes, employing the over-representation enrichment analysis at WEB-based GEne SeT AnaLysis Toolkit (2019 Version; http://webgestalt.org), a set of computational tools developed and maintained at Dr. Bing Zhang laboratory at the Baylor College of Medicine (Houston, TX, USA). Gene ID was gene symbol, and the reference set for enrichment analysis was the human genome. Additionally, the following cut-off values were considered: at least five genes for a GO category, a maximum of 2,000 genes for a GO category, ten GO categories expected from set cover, and significance level for false discovery rate (FDR) $<0.05$ among the top $10 \mathrm{GO}$ categories (Benjamini statistical test corrected by Hochberg). 


\section{Results}

\section{A. TSC1 and TSC2 protein analyses}

In the present study, we screened six HEK293T-derived cell lines with bi-allelic inactivation of the TSC1 and/or TSC2 genes (Figure III-3B) for the expression of these genes at both mRNA and protein levels. As presented in Figure III-5 (panels A-C), under DMEMplus conditions, the protein encoded by each CRISPR-Cas9mediated knocked-out gene is not detected, as expected. Knocking-out TSC1 or TSC2 genes in homozygosity does not appear to affect the expression of AKT or S6 proteins (Figure III-5, D and E), but to relate to at least $50 \%$ reduction of the partnering protein, e.g., TSC2 when TSC1 is inactivated, or TSC1 when TSC2 is knocked out (Figure III-5, B and C). 


\section{A}
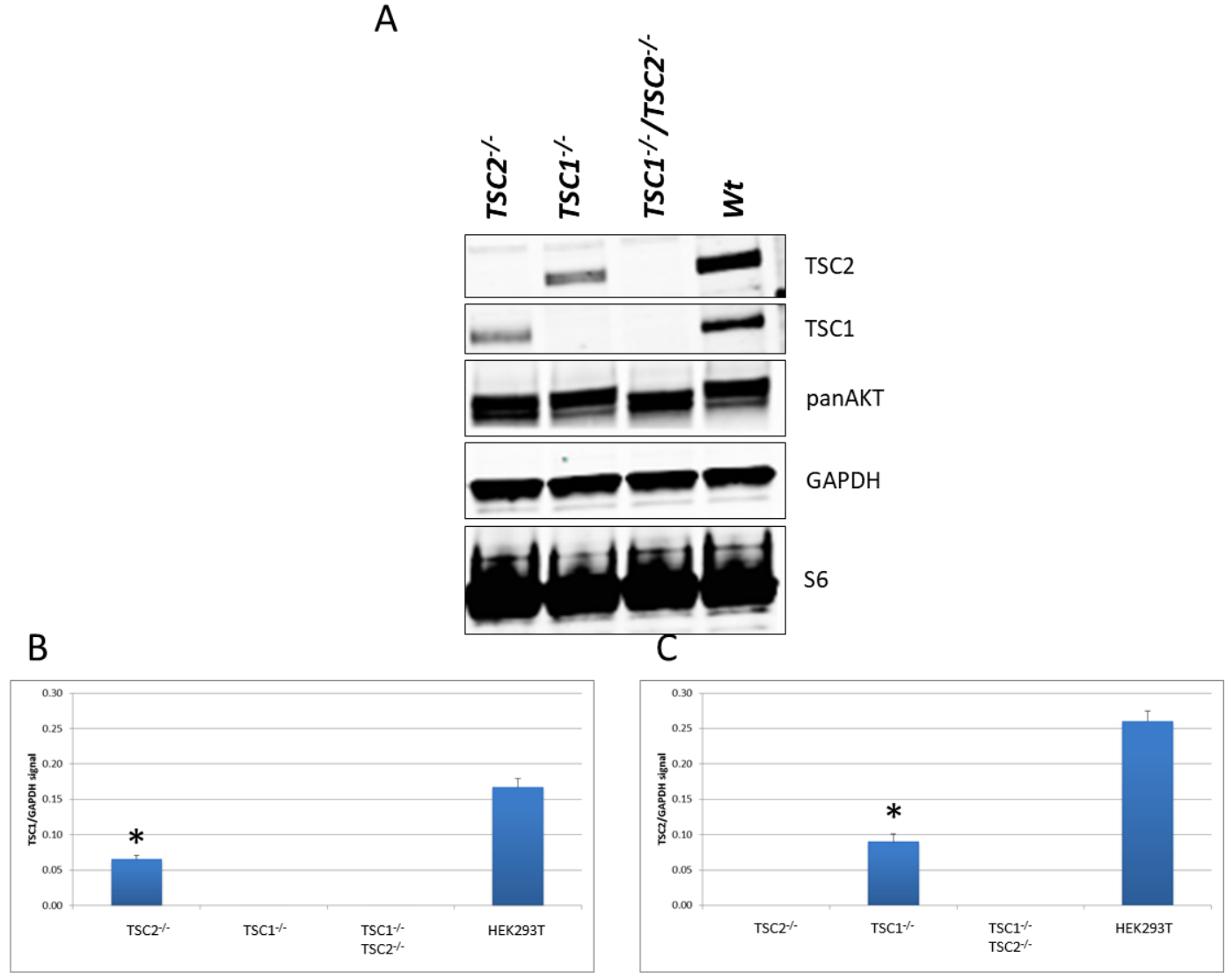

D

\section{$\mathrm{E}$}
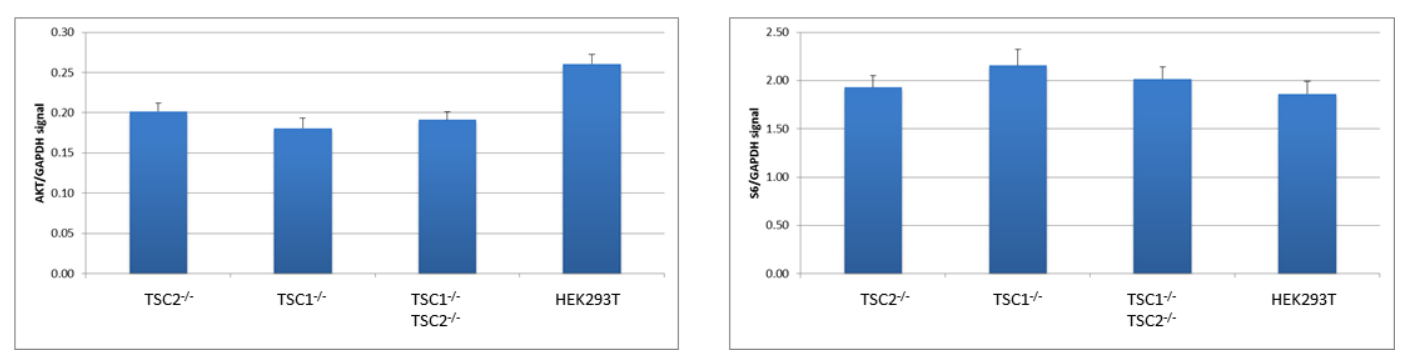

Figure III-5: TSC1, TSC2, AKT and S6 protein levels assessed by Western blotting. (A) Western blots showing protein bands for TSC2, TSC1, panAKT, GAPDH and 66 protein, as shown on the right side, respectively from HEK293T-derived cell lines $\mathrm{TSC}^{-} \%, \mathrm{TSC}_{1} \%$, $\mathrm{TSC}^{-} \%$ /TSC1\% and HEK293T reference. (B-E) Signal relative to GAPDH signal respectively for TSC1 (B), TSC2 (C), AKT (D) and S6 (E) for HEK293Tderived cell lines $\mathrm{TSC}_{2} \%, \mathrm{TSC}_{1} \%, \mathrm{TSC}^{2} \% / \mathrm{TSC}_{1} \%$ and HEK293T reference. All values were normalized to GAPDH. Asterisk represents a statistically significant difference between $\mathrm{TSC}^{\%}$ or $\mathrm{TSC}_{2} \%$ under the same culturing condition to HEK293T $(p$-value $<0.05)$.

TSC1 and TSC2 proteins were reassessed on blots in all seven cell lines in a single electrophoresis for each culturing condition. Lack of TSC1 and TSC2 proteins was reconfirmed for the respective knock-out genotypes (Figure III-6). As expected, lack of TSC1 or TSC2 protein directly related to the levels of $\mathrm{S} 6 / \mathrm{Ser}^{235} / \mathrm{Ser}^{236}$ phosphorylation, reporting mTORC1 hyperactivity (Figure III-6). For instance, all cells from DMEMplus group and the knocked-out cells from DMEMminus group had $\mathrm{S} 6 / \mathrm{Ser}^{235} / \mathrm{Ser}^{236}$ hyperphosphorylated (Figure III-6, first and second panels from second bottom row). On the contrary, DMEMminus reference HEK293T cells did not (Figure III-6, second panel from second bottom row). Addition 
of rapamycin, for one or 16 hours, down-regulated mTORC1 activity as shown by hypophosphorylation of the reporter S6/Ser $235 / \mathrm{Ser}^{236}$ (Figure III-6, third to fifth panels from second bottom row).

The PI3 kinase -Akt pathway is activated under growth conditions. Akt activity depends on two phosphorylated residues; one located in the catalytic domain (threonine 308 - Akt-Thr ${ }^{308}$ ), and the second, serine 473 (Akt-Ser ${ }^{473}$ ), in the C-terminal regulatory domain (Nicholson and Anderson 2002). Thus, Akt residues $\mathrm{Thr}^{308}$ and $\mathrm{Ser}^{473}$ are phosphorylated in response to insulin-dependent PI3K activity. As observed in figure 6, complete medium (left panel) and ten-minute insulin treatment (far right panel) of cells associate with positive blot signals for both Akt phosphorylated residues. Serum withdrawal did not change Akt activity, as reported by the blot signals observed for antibodies specific for the phosphorylated residues (Figure 6, second panel). Signals for both phosphorylated residues reporting AKT activity decreased when cells were treated overnight with rapamycin for 16 hours (Figure III-6, second right panel), though they appeared unchanged when rapamycin treatment lasted for only one hour (Figure III-6, middle panel). As Akt is upstream to the TSC1/2 complex, its residues $\mathrm{Thr}^{308}$ and $\mathrm{Ser}^{473}$ were expected to be phosphorylated in all cell lines. However, 3H9-1B1 cell line showed unphosphorylated Akt under all conditions, except for insulin treatment. 


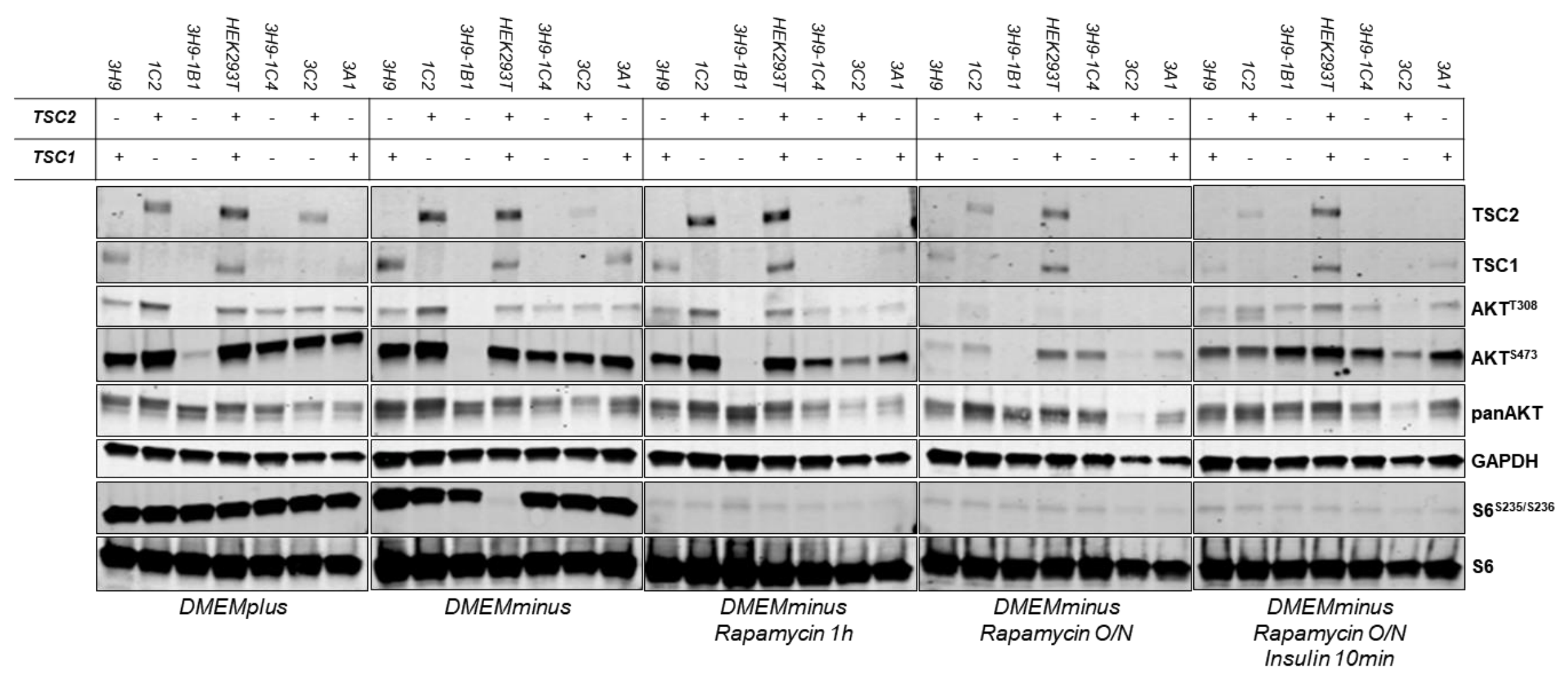

Figure III-6: Specific protein profile from genetically modified cell lines. (A) Table of cell lines showing if there is expression (+) or knock-out (-) of HEK293T TSC1 and TSC2 genes. (B) Western Blot of proteins isolated from cell lines according to table (A) expressing (+) TSC1 and TSC2 or not (-). Antibodies employed on blots detect proteins identified on the right end side (TSC2, TSC1, phosphorylated Thr ${ }^{308}$ from AKT, phosphorylated Ser ${ }^{473}$ from AKT, panAKT, GAPDH, both phosphorylated Ser ${ }^{235}$ and Ser ${ }^{236}$ from S6; and S6. The five blot panels represent distinct cell growth media and treatments, as labeled from left to right: DMEMplus, DMEMminus, DMEMminus and rapamycin for 1 hour, DMEMminus and rapamycin for 16 hours (O/N), and DMEMminus, rapamycin for 16 hours $(\mathrm{O} / \mathrm{N})$ and 10-minute treatment with insulin. 
An apparent decrease was observed for TSC1 protein amount in serum-deprived cells treated with rapamycin, in the lack of TSC2 expression (Figure III-6, cell lines 3H9 and 3A1, three right panels). Moreover, treatment with rapamycin for 16 hours also apparently reduced TSC2 protein levels in the two cell lines without TSC1 protein (1C2 and 3C2). However, gel lanes seem to have different amount of lysate protein, causing an apparent reduction of total protein levels.

\section{B. TSC1 and TSC2 mRNA analyses}

Reference HEK293T cells, under complete (DMEMplus) or minimum (DMEMminus) medium, disclosed TSC2 mRNA RPKM values respectively 2.13 and 2.70 times higher than TSC1 transcript RPKM (Figure III-7). These data show that independently on the presence of calf serum TSC2 mRNA levels are at least twice that of TSC1 mRNA in the reference HEK293T cell line, as previously reported in databases for other cell lines and tissue (Figure III-1 and Figure III-2).

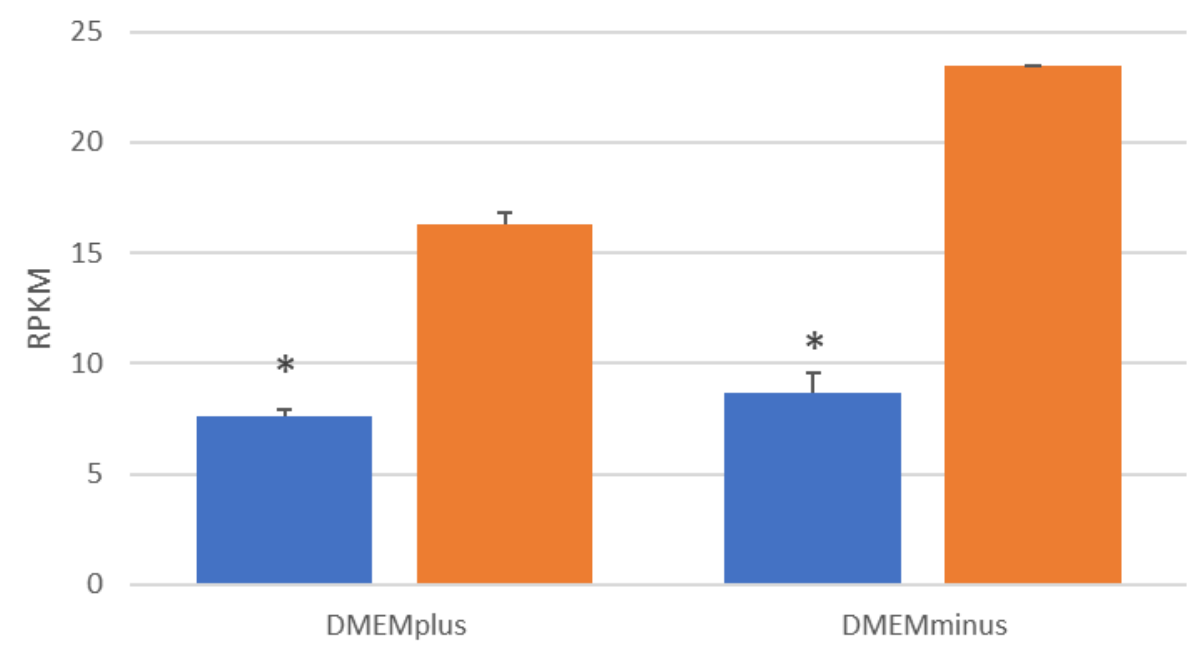

-TSC1 $\square$ TSC2

Figure III-7: TSC1 and TSC2 mRNA RPKM upon DMEMplus and DMEMminus treatments. Student $t$-test was calculated, and asterisk represents a statistically significant difference between TSC1 and TSC2 RPKM, under the same culturing condition $(p$-value $<0.05)$.

As TSC2-to-TSC1 mRNA ratio appeared similar to RNA-Seq reports on other tissues and cell lines, we subsequently analyzed their mRNA amount in HEK293T-derived cell lines for two groups, DMEMplus and DMEMminus. For that, we employed DESeq2 pairwise comparisons to the reference HEK293T cell line, a test based on binary logarithmic fold change of the gene total mRNA read count. In DMEMplus group, in the lack of the TSC1 protein (cell lines $1 \mathrm{C} 2$ and 3C2, and 3H9-1B1 and 3H9-1C4), there is a significant reduction of TSC1 mRNA (Figure III-8A). The fold change of those reductions was in average $39 \%$ (34\% to $44 \%$ ) as the binary logarithm varied between -0.60 and -0.83 (Figure III-8A). 
A

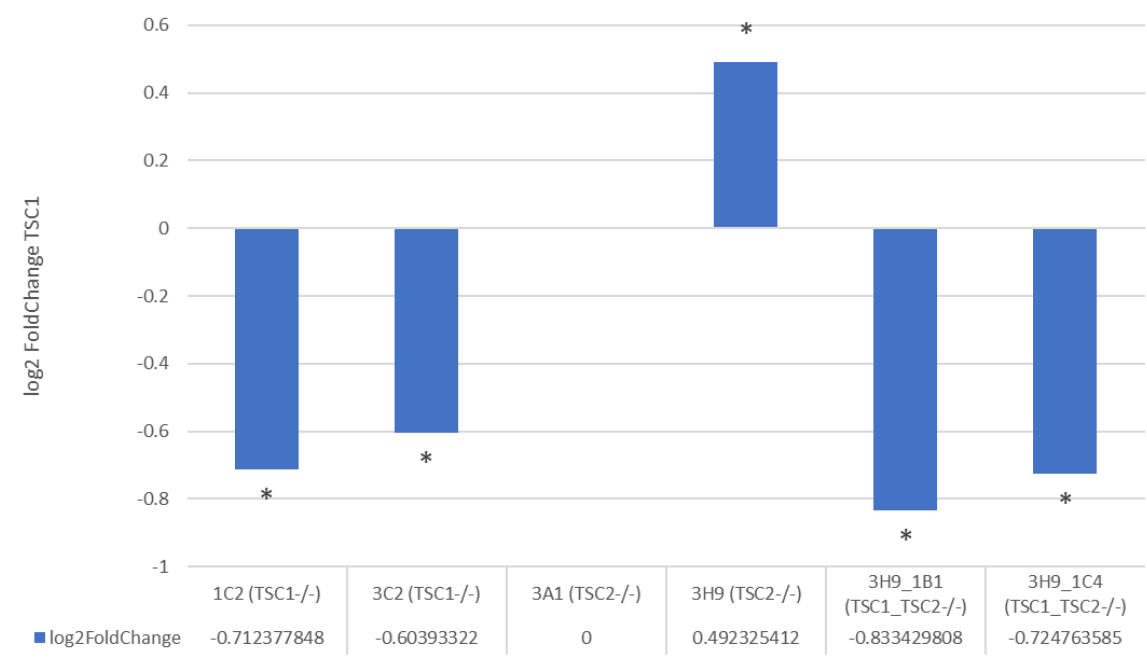

B

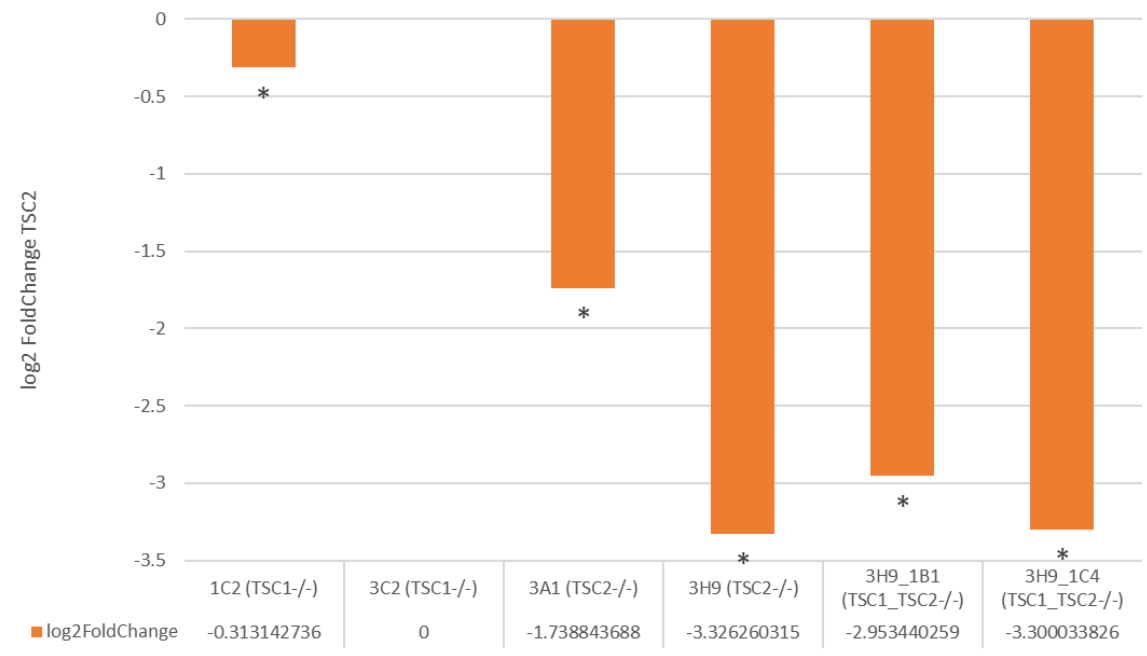

Figure III-8: Log2FoldChange of TSC1 (A) and TSC2 (B) transcript amount upon DMEMplus treatment. Asterisk $=p$-value $<0.05$.

The absence of the TSC2 protein (cell lines 3A1, 3H9, 3H9-1B1 and 3H9-1C4) related to a significant decrease in TSC2 mRNA amount (Figure III-8B). Notably, the fold change of these TSC2 mRNA reductions were considerably higher, in average $84 \%(70 \%$ to $90 \%)$ as the binary logarithm varied between -1.74 and -3.33 (Figure III-8B). The limited length of the TSC2 mRNA from the cell lines 3A1, 3H9, 3H9-1B1 and 3H9-1C4 (85\% the size of the full-length wild-type TSC2 mRNA) generates a relatively lower number of reads. Thus, when adjusted to the wild-type transcript length and data size (RKPM) a drastic reduction is expected. Remarkably, $3 \mathrm{~A} 1$ cell line, which is heterozygous for the gross TSC2 deletion, presented a less severe reduction of TSC2 mRNA (70\%; Figure III-8B).

When the effect of one protein absence was evaluated on the mRNA levels from the partnering gene, cell lines with the same knock-out genotype were discordant. Cells without the TSC1 protein had no effect or 
discrete reduction of TSC2 mRNA (1C2 and 3C2; Figure III-8B). On the other hand, cells lacking the TSC2 protein had equal amounts or significantly more TSC1 mRNA than the reference HEK293T cell line (Figure III-8A).

When the same mRNA analysis was conducted for the DMEMminus group, we observed fold-change reductions of TSC2 mRNA in the absence of TSC2 protein similar to the comparisons from the DMEMplus group, in average $88 \%$ reduction ( $78 \%$ to $93 \%$ ). The lowest reduction (78\%) was from the cell line with one allele with TSC2 gross deletion. We additionally disclosed a concordant, significant, more discrete reduction of TSC2 mRNA in both cell lines (1C2 and 3C2) that have no TSC1 protein (Figure III-9B). In the DMEMminus group, four cell lines had undetectable change in TSC1 mRNA levels in the binary logarithmic scale. One cell line without TSC1 protein (1C2) had a significant reduction of TSC1 mRNA (39\% decrease), as observed for the DMEMplus group (Figure III-9A). In another cell line, which lacks TSC2 protein (3H9), TSC1 mRNA increased nearly 70\% when compared to the reference HEK293T cell line (Figure III-9A).

A

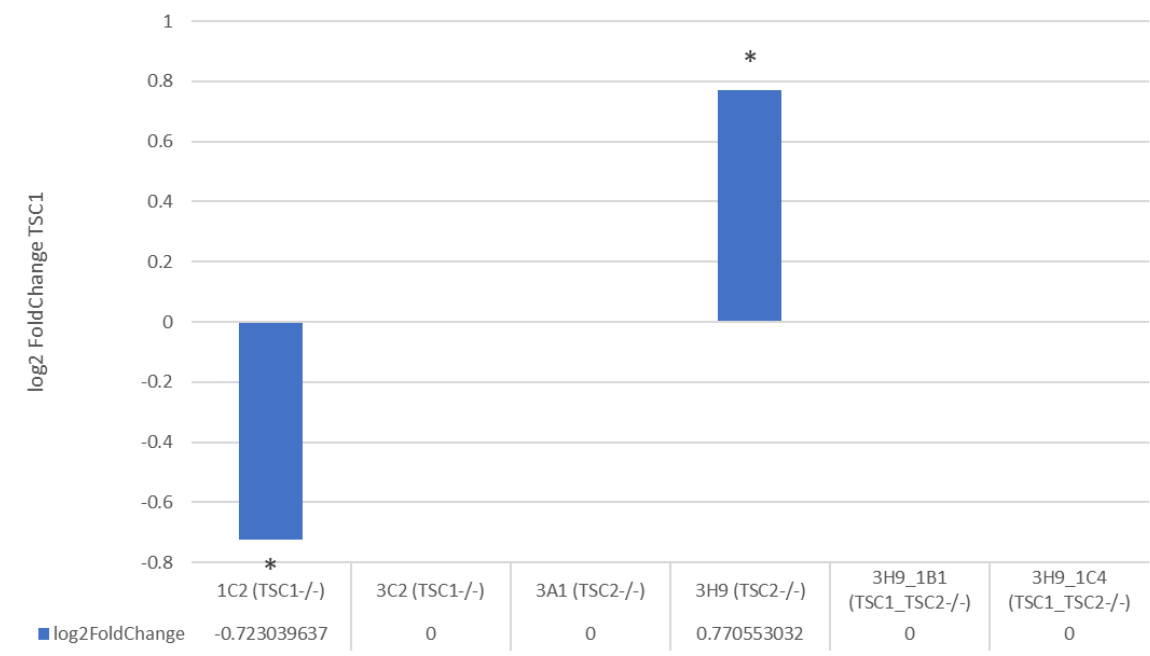

B

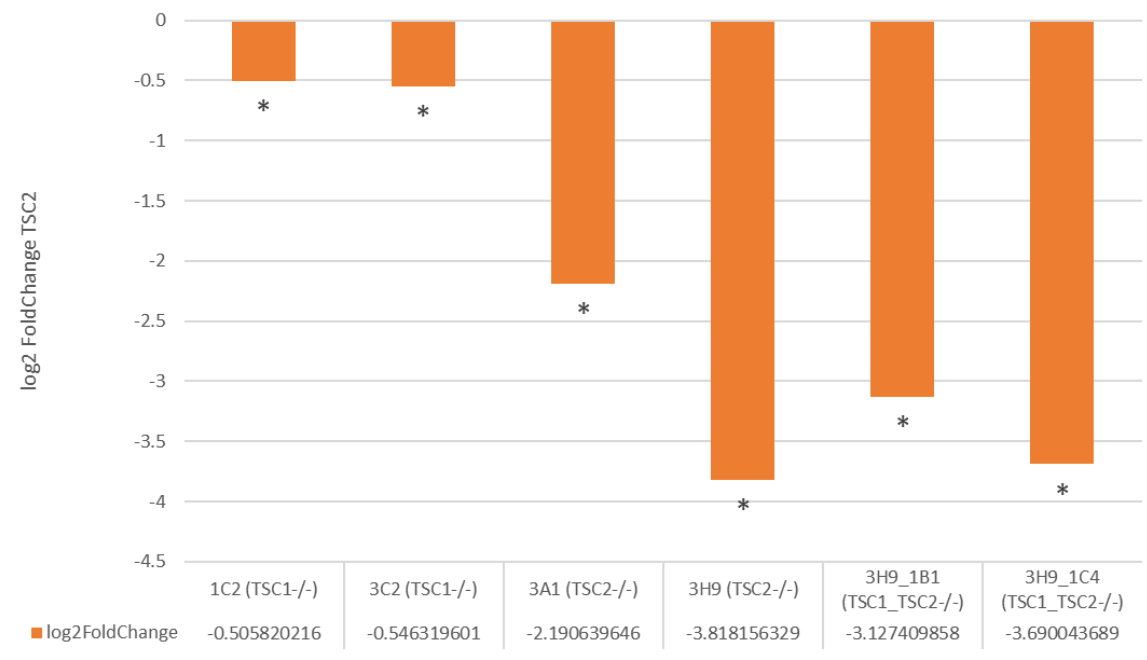

Figure III-9: Log2FoldChange of TSC1 (A) and TSC2 (B) transcript amount upon DMEMminus treatment. Asterisk $=p$-value $<0.05$. 
Table III-2: Observed TSC1 and TSC2 mRNA and protein levels for each knock-out cell line comparatively to HEK293T.

\begin{tabular}{|c|c|c|c|c|c|c|c|}
\hline \multirow{2}{*}{\multicolumn{2}{|c|}{$\begin{array}{c}\text { Knocked-out gene(s) } \\
\text { Cell line }\end{array}$}} & \multicolumn{2}{|c|}{$T S C 1$} & \multicolumn{2}{|c|}{ TSC2 } & \multicolumn{2}{|c|}{ TSC1 and TSC2 } \\
\hline & & $1 \mathrm{C} 2$ & $3 \mathrm{C} 2$ & 3A1 & $3 \mathrm{H} 9$ & 3H9-1B1 & 3H9-1C4 \\
\hline \multirow{2}{*}{ Protein } & TSC1 & - & - & $\downarrow$ & $\downarrow$ & - & - \\
\hline & TSC2 & $\downarrow$ & $\downarrow$ & - & - & - & - \\
\hline \multirow{2}{*}{$\begin{array}{l}\text { mRNA } \\
\text { (DMEMplus) }\end{array}$} & TSC1 & $\downarrow$ & $\downarrow$ & - & $\uparrow$ & $\downarrow$ & $\downarrow$ \\
\hline & TSC2 & $\downarrow$ & - & $\downarrow$ & $\downarrow$ & $\downarrow$ & $\downarrow$ \\
\hline \multirow{2}{*}{$\begin{array}{l}\text { mRNA } \\
\text { (DMEMminus) }\end{array}$} & TSC1 & $\downarrow$ & - & - & $\uparrow$ & - & - \\
\hline & $T S C 2$ & $\downarrow$ & $\downarrow$ & $\downarrow$ & $\downarrow$ & $\downarrow$ & $\downarrow$ \\
\hline
\end{tabular}

(-) unchanged or not observed; $(\downarrow)$ reduced; $(\uparrow)$ increased.

As observed in Table III-2, 1C2 was the only cell line that independently on the medium consistently decreased TSC1 and TSC2 mRNA levels. This cell line expresses no TSC1 protein. It presented decreased TSC2 protein as well as TSC1 and TSC2 mRNA amounts. The two CRISPR/Cas9-generated deletions in 1C2 are fairly small, in homozygosity. One deletion abolishes the translational start codon, and the other generates a frameshift, with no significant impact on the TSC1 mRNA length. Therefore, we selected the 1C2 cell line for further experiments, aiming at global analyses of the effects of the lack of TSC1 protein on the cell transcriptome.

\section{Global RNA analysis in the 1C2 cell line}

The number of differentially expressed genes in the 1C2 cell line compared to HEK293T (reference) gene set is indicated in Table III-3 for both DMEMminus and DMEMplus groups. 1C2 genes with less mRNA or more mRNA than the reference cell line were considered respectively down- or up-regulated. In all comparisons, Benjamini-Hochberg test was employed with FDR $<0.05$.

Table III-3: Number of genes down- or up-regulated in 1C2 cell line in regard to HEK293T in DMEMplus or DMEMminus groups.

\begin{tabular}{lcc}
\hline Groups compared & $\begin{array}{c}\text { Number of down-regulated genes and labeled } \\
\text { group ID* }\end{array}$ & $\begin{array}{c}\text { Number of up-regulated genes and labeled } \\
\text { group ID* }\end{array}$ \\
\hline 1C2 X HEK293T (DMEMminus) & $645(\mathrm{~B} 2)$ & $474(\mathrm{C} 2)$ \\
\hline 1C2 X HEK293T (DMEMplus) & $1,411(\mathrm{~B} 3)$ & $1,746(\mathrm{C} 3)$ \\
\hline
\end{tabular}

*Group ID in parenthesis

Gene ontology (GO) analysis of 645 genes down-regulated in 1C2 in minimum medium (DMEMminus) significantly grouped them in ten sets, as presented in Table III-4. The gene sets at this table is similar to the gene sets identified for down-regulated genes from DMEMplus group ( $\mathrm{N}=1,411$; Table III-5), and relate to posttranscription steps of gene expression. 
Table III-4: Gene sets significantly enriched in down-regulated genes in 1C2 cell line in regard to HEK293T, in DMEMminus group.

\begin{tabular}{lllllll}
\hline Gene Set & Description & Size & Expect & Ratio & pValue & FDR \\
\hline GO:0006401 & RNA catabolic process & 341 & 10.66 & 43.15 & 0 & 0 \\
\hline GO:0090150 & Establishment of protein localization to membrane & 313 & 97.86 & 48.03 & 0 & 0 \\
\hline GO:0006413 & Translational initiation & 192 & 60.03 & 84.96 & 0 & 0 \\
\hline GO:0070972 & Protein localization to endoplasmic reticulum & 137 & 42.83 & 95.72 & 0 & 0 \\
\hline GO:0006605 & Protein targeting & 412 & 12.88 & 38.04 & $5,55 \mathrm{E}-12$ & $9,44 \mathrm{E}-10$ \\
\hline GO:0002181 & Cytoplasmic translation & 92 & 28.76 & 73.01 & $5,84 \mathrm{E}-09$ & $8,28 \mathrm{E}-07$ \\
\hline GO:0022613 & Ribonucleoprotein complex biogenesis & 440 & 13.76 & 28.35 & $4,54 \mathrm{E}-05$ & $5,51 \mathrm{E}-03$ \\
\hline GO:0071826 & Ribonucleoprotein complex subunit organization & 245 & 76.60 & 32.64 & $2,10 \mathrm{E}-03$ & $2,22 \mathrm{E}-5$ \\
\hline GO:0034248 & Regulation of cellular amide metabolic process & 385 & 12.04 & 21.60 & $1,99 \mathrm{E}-4$ & $1,88 \mathrm{E}-2$ \\
\hline GO:0006284 & Base-excision repair & 43 & 13.44 & 52.07 & $3,39 \mathrm{E}-4$ & $2,88 \mathrm{E}-2$ \\
\hline
\end{tabular}

Table III-5: Gene sets significantly enriched in down-regulated genes in 1C2 cell line in regard to HEK293T, in DMEMplus group.

\begin{tabular}{|c|c|c|c|c|c|c|}
\hline Gene Set & Description & Size & Expect & Ratio & pValue & FDR \\
\hline GO:0022613 & Ribonucleoprotein complex biogenesis & 440 & 40.71 & 27.51 & 0 & 0 \\
\hline GO:0006401 & RNA catabolic process & 341 & 31.55 & 29.80 & 0 & 0 \\
\hline GO:0006413 & Translational initiation & 192 & 17.76 & 37.72 & 0 & 0 \\
\hline GO:0070972 & Protein localization to endoplasmic reticulum & 137 & 12.67 & 37.08 & $4,44 \mathrm{E}-12$ & $9,44 \mathrm{E}-10$ \\
\hline GO:0071826 & Ribonucleoprotein complex subunit organization & 245 & 22.66 & 26.91 & 3,63E-09 & $6,00 \mathrm{E}-07$ \\
\hline GO:0002181 & Cytoplasmic translation & 92 & 85.11 & 39.95 & 4,23E-09 & $6,00 \mathrm{E}-07$ \\
\hline GO:0016072 & rRNA metabolic process & 236 & 21.83 & 24.73 & $2,58 \mathrm{E}-06$ & $3,13 \mathrm{E}-04$ \\
\hline GO:0090150 & Establishment of protein localization to membrane & 313 & 28.96 & 21.76 & $2,25 \mathrm{E}-05$ & 2,39E-03 \\
\hline GO:0008380 & RNA splicing & 417 & 38.58 & 19.70 & $5,59 \mathrm{E}-05$ & $5,28 \mathrm{E}-03$ \\
\hline GO:0006605 & Protein targeting & 412 & 38.11 & 19.68 & 7,44E-05 & $6,33 \mathrm{E}-03$ \\
\hline
\end{tabular}

Up-regulated genes were significantly enriched in two gene sets for the DMEMminus group (Table III-6), and in at least ten GO sets for the DMEMplus group (Table III-7). Those gene sets relate to response to topologically incorrect protein and autophagic mechanism in DMEMminus group. The DMEMplus group of upregulated genes was enriched in gene sets that relate to cell growth and morphogenesis.

Table III-6 Gene sets significantly enriched in up-regulated genes in 1C2 cell line in regard to HEK293T, in DMEMminus group.

\begin{tabular}{lllllll}
\hline Gene Set & Description & Size & Expect & Ratio & pValue & FDR \\
\hline GO:0035966 & $\begin{array}{l}\text { Response to topologically incorrect } \\
\text { protein }\end{array}$ & 188 & 43.417 & 36.85 & $7,63 \mathrm{E}-6$ & $6,48 \mathrm{E}-3$ \\
\hline GO:0061919 & $\begin{array}{l}\text { Process utilizing autophagic } \\
\text { mechanism }\end{array}$ & 473 & 10.923 & 22.89 & $1,07 \mathrm{E}-4$ & $4,56 \mathrm{E}-2$ \\
\hline
\end{tabular}


Table III-7: Gene sets significantly enriched in up-regulated genes in 1C2 cell line in regard to HEK293T, in DMEMplus group.

\begin{tabular}{lllllll}
\hline Gene Set & Description & Size & Expect & Ratio & pValue & FDR \\
\hline GO: 0034330 & Cell junction organization & 285 & 19.73 & 23.32 & $5,77 \mathrm{E}-04$ & $4,90 \mathrm{E}-5$ \\
\hline GO: 0022604 & Regulation of cell morphogenesis & 473 & 32.74 & 18.94 & $7,86 \mathrm{E}-03$ & $3,34 \mathrm{E}-4$ \\
\hline GO: 2000147 & Positive regulation of cell motility & 493 & 34.12 & 18.17 & $3,17 \mathrm{E}-6$ & $8,45 \mathrm{E}-4$ \\
\hline GO: 0010975 & $\begin{array}{l}\text { Regulation of neuron projection } \\
\text { development }\end{array}$ & 475 & 32.88 & 18.25 & $3,97 \mathrm{E}-6$ & $8,45 \mathrm{E}-4$ \\
\hline GO: 0060560 & $\begin{array}{l}\text { Developmental growth involved in } \\
\text { morphogenesis }\end{array}$ & 225 & 15.57 & 22.47 & $5,16 \mathrm{E}-6$ & $8,78 \mathrm{E}-4$ \\
\hline GO: 0090066 & $\begin{array}{l}\text { Regulation of anatomical structure } \\
\text { size }\end{array}$ & 487 & 33.71 & 17.80 & $8,74 \mathrm{E}-6$ & $1,12 \mathrm{E}-4$ \\
\hline GO: 0045785 & Positive regulation of cell adhesion & 392 & 27.132 & 18.797 & $9,25 \mathrm{E}-6$ & $1,12 \mathrm{E}-3$ \\
\hline GO: 0048638 & Regulation of developmental growth & 314 & 21.733 & 19.325 & $2,91 \mathrm{E}-5$ & $3,09 \mathrm{E}-3$ \\
\hline GO: 0048732 & Gland development & 434 & 30.039 & 17.644 & $3,70 \mathrm{E}-5$ & $3,49 \mathrm{E}-3$ \\
\hline GO: 0045926 & Negative regulation of growth & 250 & 17.304 & 20.227 & $5,17 \mathrm{E}-5$ & $4,39 \mathrm{E}-3$ \\
\hline
\end{tabular}

As shown in Table III-3, we labeled as B2, B3, C2, and C3 the sets of differentially expressed genes from comparisons between 1C2 and HEK293T cell lines, if they were respectively down-regulated in DMEMminus or DMEMplus groups, or up-regulated in DMEMminus or DMEMplus groups. These gene group identifications were used to show in Table III-8 the intersections that were generated between gene sets from two experimental groups. The number of retrieved genes is indicated in the same table.

Table III-8: Number of genes observed when groups assigned in Table III-3 were intersected.

\begin{tabular}{lc}
\hline Intersected groups & Number of genes from intersection or intersection subtracted \\
\hline $\mathrm{B} 2 \cap \mathrm{B} 3$ & 513 \\
\hline $\mathrm{C} 2 \cap \mathrm{C} 3$ & 326 \\
\hline $\mathrm{B} 2-(\mathrm{B} 2 \cap \mathrm{B} 3)$ & 133 \\
\hline $\mathrm{B} 3-(\mathrm{B} 2 \cap \mathrm{B} 3)$ & 1,234 \\
\hline $\mathrm{C} 2-(\mathrm{C} 2 \cap \mathrm{C} 3)$ & 149 \\
\hline $\mathrm{C} 3-(\mathrm{C} 2 \cap \mathrm{C} 3)$ & 1,086 \\
\hline B2: down-regulated genes in DMEMminus group from 1C2 X HEK293T comparison \\
B3: down-regulated genes in DMEMplus group from 1C2 X HEK293T comparison \\
C2: up-regulated genes in DMEMminus group from 1C2 X HEK293T comparison \\
C3: up-regulated genes in DMEMplus group from 1C2 X HEK293T comparison
\end{tabular}

The intersections between down-regulated gene sets (B2 $\cap$ B3) represent significantly downregulated genes independently on the presence of serum. As shown in Table III-9, the twelve gene sets enriched in this intersection group ( $N=513$ ) relates to post-transcritptional steps of gene expression, notably mRNA translation, and ATP generation. Thus, these biological processes appear down-regulated in the lack of the TSC1 protein, independently on culture serum (trophic factors). Differentially expressed genes uniquely detected in the presence of serum were enriched in gene sets also directly related to gene expression, 
however, mostly to the nuclear steps of gene expression (Table III-10). Down-regulated genes detected independently on serum in DMEMminus but not in DMEMplus samples (B2 - (B2 $\cap$ B3), $N=133$ ) were not significantly enriched in gene sets.

Table III-9: Gene sets significantly enriched in down-regulated genes in 1C2 cell line in regard to HEK293T, independently on the serum presence.

\begin{tabular}{lllllll}
\hline Gene Set & Description & Size & Expect & Ratio & pValue & FDR \\
\hline GO:0090150 & $\begin{array}{l}\text { Establishment of protein localization to } \\
\text { membrane }\end{array}$ & 313 & 79.68 & 55.22 & 0 & \\
\hline GO:0006413 & Translational initiation & 192 & 48.88 & 98.20 & 0 & 0 \\
\hline GO:0070972 & Protein localization to endoplasmic reticulum & 137 & 34.88 & 10.90 & 0 & 0 \\
\hline GO:0006401 & RNA catabolic process & 341 & 86.81 & 47.23 & $1,11 \mathrm{E}-12$ & $2,36 \mathrm{E}-10$ \\
\hline GO:0006605 & Protein targeting & 412 & 10.49 & 41.95 & $5,55 \mathrm{E}-12$ & $9,44 \mathrm{E}-10$ \\
\hline GO:0002181 & Cytoplasmic translation & 92 & 23.42 & 85.39 & $1,24 \mathrm{E}-09$ & $1,76 \mathrm{E}-07$ \\
\hline GO:0022613 & Ribonucleoprotein complex biogenesis & 440 & 11.20 & 32.14 & $6,45 \mathrm{E}-06$ & $7,84 \mathrm{E}-04$ \\
\hline GO:0071826 & Ribonucleoprotein complex subunit organization & 245 & 62.37 & 36.88 & $7,53 \mathrm{E}-04$ & $8,00 \mathrm{E}-6$ \\
\hline GO:0009141 & Nucleoside triphosphate metabolic process & 304 & 77.39 & 24.55 & $3,02 \mathrm{E}-4$ & $2,85 \mathrm{E}-2$ \\
\hline GO:0009123 & Nucleoside monophosphate metabolic process & 321 & 81.72 & 23.25 & $5,91 \mathrm{E}-4$ & $5,02 \mathrm{E}-2$ \\
\hline GO:0006091 & Generation of precursor metabolites and energy & 469 & 11.940 & 20.101 & $9,64 \mathrm{E}-4$ & $7,44 \mathrm{E}-2$ \\
\hline GO:0016072 & rRNA metabolic process & 236 & 60.080 & 24.967 & $1,07 \mathrm{E}-3$ & $7,63 \mathrm{E}-2$ \\
\hline
\end{tabular}

Table III-10: Gene sets significantly enriched in down-regulated genes in 1C2 cell line in regard to HEK293T, detected in DMEMplus but not in DMEMminus samples

\begin{tabular}{lllllll}
\hline Gene Set & Description & Size & Expect & Ratio & pValue & FDR \\
\hline GO:0022613 & Ribonucleoprotein complex biogenesis & 440 & 29.50 & 25.76 & $1,33 \mathrm{E}-10$ & $1,13 \mathrm{E}-07$ \\
\hline GO:0006397 & mRNA processing & 487 & 32.65 & 21.74 & $3,64 \mathrm{E}-06$ & $1,55 \mathrm{E}-03$ \\
\hline GO:0008380 & RNA splicing & 417 & 27.96 & 22.53 & $8,74 \mathrm{E}-06$ & $2,48 \mathrm{E}-03$ \\
\hline GO:0006403 & RNA localization & 228 & 15.29 & 27.47 & $1,70 \mathrm{E}-05$ & $3,61 \mathrm{E}-03$ \\
\hline GO:0006401 & RNA catabolic process & 341 & 22.86 & 23.18 & $7,26 \mathrm{E}-05$ & $1,23 \mathrm{E}-6$ \\
\hline GO:1903311 & Regulation of mRNA metabolic process & 266 & 17.84 & 24.11 & $6,12 \mathrm{E}-04$ & $8,66 \mathrm{E}-6$ \\
\hline GO:0016072 & rRNA metabolic process & 236 & 15.82 & 24.64 & $1,39 \mathrm{E}-03$ & $1,68 \mathrm{E}-5$ \\
\hline GO:0010608 & Posttranscriptional regulation of gene expression & 486 & 32.59 & 19.33 & $3,15 \mathrm{E}-03$ & $3,34 \mathrm{E}-5$ \\
\hline GO:0051169 & Nuclear transport & 356 & 23.87 & 20.95 & $4,94 \mathrm{E}-03$ & $4,66 \mathrm{E}-5$ \\
\hline GO:0071826 & Ribonucleoprotein complex subunit organization & 245 & 16.43 & 23.13 & $1,03 \mathrm{E}-6$ & $8,76 \mathrm{E}-5$ \\
\hline
\end{tabular}

There was no significant enrichment in gene sets for up-regulated genes in 1 C2 cell line when compared to HEK293, independently on the serum presence ( $C 2 \cap C 3, N=326$ ). Gene sets such as response to topologically incorrect protein and regulation of protein stability were enriched in up-regulated genes in 
DMEMminus but not in DMEMplus (Table III-11). Enriched gene sets for genes up-regulated in DMEMplus but not DMEMminus samples relate to cell morphogenesis (Table III-12).

Table III-11: Gene sets significantly enriched in up-regulated genes in 1C2 cell line in regard to HEK293T, detected in DMEMminus but not in DMEMplus samples.

\begin{tabular}{lllllll}
\hline Gene Set & Description & Size & Expect & Ratio & pValue & FDR \\
\hline GO:0035966 & Response to topologically incorrect protein & 188 & 14.47 & 62.19 & $1,45 \mathrm{E}-5$ & $1,23 \mathrm{E}-2$ \\
\hline GO:0031647 & Regulation of protein stability & 267 & 20.55 & 48.65 & $4,02 \mathrm{E}-5$ & $1,70 \mathrm{E}-2$ \\
\hline
\end{tabular}

Table III-12: Gene sets significantly enriched in up-regulated genes in 1 C2 cell line in regard to HEK293T, detected in DMEMplus but not in DMEMminus samples

\begin{tabular}{lllllll}
\hline Gene Set & Description & Size & Expect & Ratio & pValue & FDR \\
\hline GO:0034330 & Cell junction organization & 285 & 15.34 & 26.08 & $2,43 \mathrm{E}-04$ & $2,06 \mathrm{E}-5$ \\
\hline GO:0022604 & Regulation of cell morphogenesis & 473 & 25.46 & 19.64 & $3,70 \mathrm{E}-6$ & $1,57 \mathrm{E}-3$ \\
\hline GO:0060560 & Developmental growth involved in morphogenesis & 225 & 12.11 & 23.95 & $1,12 \mathrm{E}-5$ & $3,18 \mathrm{E}-3$ \\
\hline GO:0048638 & Regulation of developmental growth & 314 & 16.90 & 21.30 & $1,50 \mathrm{E}-5$ & $3,18 \mathrm{E}-3$ \\
\hline GO:0043087 & Regulation of GTPase activity & 472 & 25.40 & 18.50 & $3,40 \mathrm{E}-5$ & $5,79 \mathrm{E}-3$ \\
\hline GO:2000147 & Positive regulation of cell motility & 493 & 26.53 & 18.09 & $4,97 \mathrm{E}-5$ & $7,04 \mathrm{E}-3$ \\
\hline GO:0045785 & Positive regulation of cell adhesion & 392 & 21.10 & 18.96 & $7,60 \mathrm{E}-5$ & $8,59 \mathrm{E}-3$ \\
\hline GO:0010975 & Regulation of neuron projection development & 475 & 25.56 & 17.99 & $8,08 \mathrm{E}-5$ & $8,59 \mathrm{E}-3$ \\
\hline GO:0031589 & Cell-substrate adhesion & 332 & 17.87 & 19.59 & $1,11 \mathrm{E}-4$ & $1,04 \mathrm{E}-2$ \\
\hline GO:0090066 & Regulation of anatomical structure size & 487 & 26.21 & 17.55 & $1,45 \mathrm{E}-4$ & $1,23 \mathrm{E}-2$ \\
\hline
\end{tabular}

When only the $1 C 2$ cell line was considered for global RNA analyses, and the groups DMEMminus and DMEMplus (reference) were compared, we retrieved 72 and 51 genes that were respectively down - and upregulated in 1C2 DMEMminus group. Gene sets that revealed a significant enrichment are presented in Table III-13 and Table III-14. We observed that response to topologically incorrect protein is both down- and upregulated. Response to endoplasmic reticulum stress is down-regulated, whereas protein folding and response to temperature stress are up-regulated, consistently with the role of TSC1 protein as heat-shock protein cochaperone. 
Table III-13: Gene sets significantly enriched in down-regulated genes in DMEMminus group in regard to DMEMplus, in the 1 C2 cell line.

\begin{tabular}{lllllll}
\hline Gene Set & Description & Size & Expect & Ratio & pValue & FDR \\
\hline GO:0034976 & Response to endoplasmic reticulum stress & 268 & 10.47 & 76.22 & $9,07 \mathrm{E}-6$ & $7,71 \mathrm{E}-3$ \\
\hline GO:0006730 & One-carbon metabolic process & 25 & 0.10 & 30.64 & $1,23 \mathrm{E}-4$ & $3,49 \mathrm{E}-2$ \\
\hline GO:0035966 & Response to topologically incorrect protein & 188 & 0.73 & 81.49 & $9,04 \mathrm{E}-5$ & $3,49 \mathrm{E}-2$ \\
\hline GO:0070972 & Protein localization to endoplasmic reticulum & 137 & 0.54 & 93.18 & $1,94 \mathrm{E}-4$ & $4,13 \mathrm{E}-2$ \\
\hline
\end{tabular}

Table III-14: Gene sets significantly enriched in up-regulated genes in DMEMminus group in regard to DMEMplus, in the 1 C2 cell line.

\begin{tabular}{lllllll}
\hline Gene Set & Description & Size & Expect & Ratio & pValue & FDR \\
\hline GO:0035966 & Response to topologically incorrect protein & 188 & 0.49510 & 18.178 & $1,08 \mathrm{E}-05$ & $6,22 \mathrm{E}-03$ \\
\hline GO:0051131 & Chaperone-mediated protein complex assembly & 20 & 0.052671 & 94.929 & $1,46 \mathrm{E}-05$ & $6,22 \mathrm{E}-03$ \\
\hline GO:0006457 & Protein folding & 210 & 0.55304 & 12.657 & $1,09 \mathrm{E}-6$ & $3,09 \mathrm{E}-4$ \\
\hline GO:0009266 & Response to temperature stimulus & 201 & 0.52934 & 11.335 & $1,30 \mathrm{E}-5$ & $2,76 \mathrm{E}-3$ \\
\hline GO:0070841 & Inclusion body assembly & 22 & 0.057938 & 51.780 & $2,51 \mathrm{E}-5$ & $4,27 \mathrm{E}-3$ \\
\hline GO:0046677 & Response to antibiotic & 316 & 0.83220 & 72.098 & $1,62 \mathrm{E}-4$ & $2,29 \mathrm{E}-2$ \\
\hline GO:0044839 & Cell cycle G2/M phase transition & 213 & 0.56094 & 89.136 & $2,27 \mathrm{E}-4$ & $2,75 \mathrm{E}-2$ \\
\hline GO:0071559 & Response to transforming growth factor beta & 238 & 0.62678 & 79.773 & $3,78 \mathrm{E}-4$ & $4,02 \mathrm{E}-2$ \\
\hline
\end{tabular}




\section{Discussion}

\section{A. Cell line characterization}

In this study, we employed six cell lines derived from HEK293T cell line by CRISPR-Cas9 editing of the TSC1 (chromosome 9q34.1) and/or TSC2 (chromosome 16p13.3) genes. HEK293 cell line has been first obtained after immortalization by adenovirus transduction of primarily cultured human embryonic kidney (HEK) cells (Graham, Smiley et al. 1977). Although the identity of the cell that originally derived the HEK293 line is unknown, later analyses disclosed that HEK293 cells exhibit some adrenal cell properties, including neuronal-like morphophysiology (Lin, Boone et al. 2014). Therefore, the original HEK293 line should not be considered as a kidney-derived cell line, but a complex, heterogeneous cell line, likely derived from cells of the adrenal glands, which are anatomically related to the kidneys (Lin, Boone et al. 2014). Among further genomic modifications these cells have undergone, HEK293T cell line, employed in our study, originated upon SV40 transfection of the HEK293 cell line (Rio, Clark et al. 1985). HEK293T presents a combination of karyotypes with some chromosomes in three copies, leading to the classification of the cell line as hypotriploid or pseudotriploid (Lin, Boone et al. 2014). In 19 HEK293T chromosome metaphases examined by Lin, Boone et al. (2014), six cells presented $t(9,17)$ translocations, five had duplicated chromosome 9 short arm, two cells had deleted 9q, one cell had a 9q duplication, and two cells had a translocation involving chromosome 16 $(t(16,21)$ and $t(16,22)$. Although Lin, Boone et al. (2014) did not demonstrate the translocation breakpoints nor 9q34.1 and 16p13.3 trisomy in HEK293T cells, their data tend to suggest a situation of eventual di- or monosomy for the TSC1 and TSC2 genes. As TSC1 and TSC2 genes are considered haplosufficient in nonneuronal cells, we believe that HEK293T chromosome 9 and 16 ploidies are not a concern for the present study.

CRISPR-Cas9-edited HEK293T cell lines analyzed in the present study have been previously produced in the Nellist laboratory (Erasmus Medical Centre, Rotterdam, The Netherlands), and have TSC1 and TSC2 genotypes indicated in Figure III-3. Our Western blot analyses have shown that all cell lines that had either gene inactivated did not express the respective protein (Figure III-5 and Figure III-6), and had mTORC1 hyperactivated, as presented in the functional assay by S6-phosphorylation reporter (Figure III-6). Hyperactivated mTOR was observed under both DMEMplus and DMEMminus conditions, similarly to reports from the literature (Tee, Fingar et al. 2002, Garami, Zwartkruis et al. 2003). Amino acids, such as leucine and arginine, activate mTORC1 independently on growth factors from serum in cells where TSC1 or TSC2 is knocked out (Byfield, Murray et al. 2005, Nobukuni, Joaquin et al. 2005, Smith, Finn et al. 2005). As DMEM minimum medium contains 15 out of the 20 amino acids, it is believed that the growth factor-independent, amino-aciddependent mTORC1 activation pathway should be undertaken. This pathway is regulated by the Rag GTPases, which allow mTORC1 to interact with the lysosome membrane. Therefore, in DMEMminus conditions, cells 
negative for TSC1 or TSC2 present hyperphosphorylated S6-Ser ${ }^{235}$ and Ser ${ }^{236}$ (Figure III-6, second panel). Under conditions of amino acid depletion, Rag GTPases recruit TSC2 to the lysosome, interacting with Rheb. mTORC1 is thus released from the lysosome and becomes inactivated (Bar-Peled, Schweitzer et al. 2012). As seen in Figure III-6 (third and fourth panels), mTORC1 activity was inhibited upon rapamycin treatment for one or 16 hours, as shown by hypophosphorylation of the reporter $\mathrm{S6}-\mathrm{Ser}^{235} / \mathrm{Ser}^{236}$.

Upon insulin or insulin-like growth factor (IGF) binding, the insulin/IGF receptor becomes activated by auto-phosphorylation, starting a signalling cascade that activates the PI3 kinase (PI3K)-Akt pathway. PI3K activity on membrane lipids is essential for Akt and PDK1 (phosphoinositide-dependent kinase 1) translocation to the cell membrane. PDK1 has been demonstrated to phosphorylate Akt-Thr ${ }^{308}$, thus inhibiting the TSC1/2 complex (Kwiatkowski and Manning 2005). On the other hand, considerable efforts have been made to understand the roles of different kinases shown to phosphorylate Akt-Ser ${ }^{473}$. For instance, mTORC2 (mechanistic target of rapamycin complex 2) has been reported to phosphorylate Akt-Ser ${ }^{473}$ under a variety of physiological conditions, and to facilitate phosphorylation of AKT-Thr ${ }^{308}$ by PDK1 (Sarbassov, Guertin et al. 2005). Similarly to mTORC1, the large molecular complex mTORC2 contains mTOR and mLST8 (mammalian lethal with Sec13 protein 8), but differs from the former complex as they present different additional partnering proteins, subcellular localization, functions and sensitivity to rapalogs. MTORC2 phosphorylates several members of protein kinase families A, B (Akt), C and G, affecting cytoskeleton remodelling, ion transport and cell survival, growth, proliferation (Reviewed by Menon, 2018).

We observed that serum starvation for 16 hours did not apparently affect the phosphorylation status of Akt residues $\mathrm{Thr}^{308}$ and Ser ${ }^{473}$ not even when followed by one-hour treatment with rapamycin (Figure 6). It is as yet difficult to explain the lack of effect of serum deprivation on Akt dephosphorylation. However, as there should be a reciprocal relation between Akt and the two mTOR complexes, different possibilities could be raised, such as inhibition of specific phosphatases, a delayed response to repress mTORC2 or susceptibility of Akt $\mathrm{Thr}^{308}$ and Ser ${ }^{473}$ to additional kinases.

The $\mathrm{TSC}^{-1}$, $\mathrm{TSC}^{-1-}$ 3H9-1B1 cell line had AKT hypophosphorylated in all treatments, a situation restored upon insulin treatment. A marked reduction in Akt activation was reported in $T s c 2^{-1-} / T p 53^{-/-}$and $\mathrm{Tsc1}^{-/-}$cells in response to serum (Zhang, Cicchetti et al. 2003). The combination of double knock-out 3H9-1B1 cell line and the intrinsic inhibition of p53 in HEK293T (Lilyestrom, Klein et al. 2006) could explain why this cell line did not respond to Akt kinases (Figure III-6). However, 3H9-1B1 cell line did not considerably differ from 3H9-1C4, except for Akt phosphorylation status.

Knocking-out TSC1 or TSC2 genes by CRISPR-Cas9 genome edition did not affect the overall protein levels of upstream AKT protein or downstream S6 protein (Figure III-6). The TSC2 levels in $\mathrm{TSC1}^{-1-}$ cells were reduced relative to the wild-type cell line HEK293T; likewise, the protein levels of TSC1 in TSC2\%- cells (Table 
III-2). Our data do not corroborate observations that the knockout of Tsc2 maintains levels of Tsc1 (Zhang, Gao et al. 2003, Pollizzi, Malinowska-Kolodziej et al. 2009). However, embryonic fibroblasts obtained from murine Tsc1 knockout model (MEF Tsc1-/-) showed reduced levels of Tsc2 (Astrinidis, Senapedis et al. 2006). TSC1 transient transfection increased endogenous levels of TSC2 in wild-type cells, and transient co-transfection of TSC1 with TSC2 in the same cell type increased TSC2 levels (Benvenuto, Li et al. 2000). These observations can be attributed to the Tsc1 property as a co-chaperone of Hsp90 and properly folding of Tsc2 (Woodford, Sager et al. 2017). The absence of TSC1 in human cell line might have the same effects as in mouse cells, what could inhibit the folding of TSC2, thus leading to its ubiquitination and degradation. In the same way, low levels of TSC2 in knocked-out cell lines should lead to ubiquitination and degradation of TSC1. Part of non-degraded TSC1 could accumulate in the cell as partially unfolded, self-aggregated protein, as suggested by Nellist, van Slegtenhorst et al. (1999). As TSC1 protein has been considered a chaperone-allied protein, this scenario considering both protein degradation and aggregation could be closely examined to answer as well if other protein species are also unfolded and present in the TSC1 aggregates.

\section{B. TSC1 and TSC2 gene expression regulation}

The expression of TSC2 has been observed to be higher than that of the TSC1 gene at the mRNA level (Figure III-1 and 2), independently whether cells are growing under complete or minimum medium (Figure III-7). However, the half-life of mRNA depends on transcription activity as well as decay mechanisms. Thus it is recommended to refer to as alteration in MRNA amount or levels, as the responsible mechanisms cannot be inferred without further experiments. Furthermore, one cannot infer a quantitative correlation between mRNA and the respective protein taking into account the mRNA amount, as there are various posttranscriptional steps regulating gene expression.

Regardless of medium conditions, the reduction of TSC2 mRNA expression has been more pronounced than those in TSC1 mRNA levels. This higher decrease in expression of TSC2 may be due to the genomic edition, which subtracted nearly $4.8 \mathrm{~Kb}$ of TSC2 coding sequence (Figure III-3B). Consequently, the $\mathrm{TSC}^{-1-}$ cell lines should provide significantly lower RNA-Seq TSC2 read count, and thus they do not constitute an appropriate model for TSC1/2 gene regulation studies. Furthermore, the unaffected TSC1 mRNA levels in the DMEMminus group (similar to HEK293T TSC1 mRNA levels) suggest that the TSC1 mRNA down-regulation should be sensitive to the presence of growth factors, TSC1 and TSC2 proteins. As mTORC1 has been functionally involved in transcriptional control as well translational regulation, it should be interesting to study if rapamycin treatment affects TSC1 mRNA in the 1C2 cell line. We also disclosed a discrete reduction of TSC2 mRNA in both cell lines that have no TSC1 protein (Figure 9B) under minimum medium condition.

The extension of the mRNA $5^{\prime}$-untranslated regions (5'-UTR) is similar for human TSC1 (234 bp) and TSC2 (200 bp) mRNA. The 230-bp 5'UTR of mouse Tsc1 is $88 \%(202 / 230)$ similar to the human orthologous 
5'UTR. The 119-bp 5'UTR of mouse Tsc2 is 70\% (83/119) similar to the $5^{\prime}$ UTR of the human orthologue. However, human TSC1 (4,887 bp) and TSC2 (109 bp) 3'- untranslated region (3'-UTR) lengths are significantly different. Human and mouse Tsc1 3'-UTR length and sequence are conserved, the latter containing 3,999 bp. On the other hand, although human and mouse Tsc2 3'-UTRs have similar lengths, the latter with $131 \mathrm{bp}$, the sequences display lower similarity $(51 \%, 56 / 109)$. The longer TSC1 3 '-UTR may infer that this mRNA is more responsive to translational regulation and more stable than TSC2 mRNA, as it may harbor cis regulatory motifs in that transcribed segment. Furthermore, changes in mRNA amount may build a feedback control loop on TSC gene transcription. Studies based on quantitative RT-PCR of the TSC1 and TSC2 CDNA, combined with the use of chemical transcriptional, translational or proteasomal inhibitors are likely to clarify the type of regulation to which TSC mRNA and proteins should be submitted. The data presented here, along with the anatomy of the TSC1 gene, suggest that cis elements in the mRNA 3'-UTR region could regulate the stability of the transcript. Finally, if RNA decay and translation appear to regulate the steady state of TSC1 protein and mRNA, it would be worth screening TSC1 mRNA for cis-regulating motifs.

\section{Protein stress response in TSC1-negative cell line}

$\mathrm{TSC}^{-1-} 1 \mathrm{C} 2$ cell line has one deletion encompassing the translational start codon and another causing frameshift, basically with no impact on TSC1 RNA length. Considering that more than $80 \%$ of TSC patients have point or small indel pathogenic variants (Niida, Lawrence-Smith et al. 1999, van Slegtenhorst, Verhoef et al. 1999, Dabora, Jozwiak et al. 2001, Au, Williams et al. 2007), the 1C2 cell line should be a good model study loss-of-function TSC1 mutations. Although it was not an objective of the present work, we were provided with RNA-Seq from the seven cell lines under different growth conditions. As the 1C2 cell line appeared to consistently affect TSC1 and TSC2 mRNA and protein, its RNA-Seq data were analyzed under complete or minimum medium in comparison to HEK293T reference cell line.

Under growth conditions, 1C2 down-regulated mRNAs related to posttranscriptional steps of gene expression, notably RNA processes and translation (Table III-10), and was not considerably different from gene sets enriched in down-regulated genes for starvation conditions (Table III-4). However, the latter group was more represented by translation-associated GOs. It is important to note that the differentially expressed genes have not been identified or studied on an individual basis. Therefore, it is possible that translation-associated GOs be enriched in translation repression genes, which are expected to be down-regulated when mTOR is hyperactivated (Goncharova, Goncharov et al. 2002).

Likewise, it is important to put in evidence that the GO response to unfolded proteins has been identified in both down-regulated and up-regulated gene sets, inferring that, if truly positive evidences, a detailed analysis of each gene function is advised, as positive and negative regulatory functions could be represented. These data, as well as the gene sets for the ER stress response are consistent with the role of 
TSC1 as HSP90 co-chaperone (Woodford, Sager et al. 2017, Sager, Woodford et al. 2018, Sager, Woodford et al. 2018).

The DMEMplus group presented up-regulated genes significantly enriched in cell growth and morphogenesis sets while, in DMEMminus set, topologically incorrect protein and autophagic mechanisms were enriched. Cell size phenotypes have been observed in $T s 1^{-1}$ cells from Drosophila melanogaster that dramatically increased in size, yet differentiated normally (Potter, Huang et al. 2001). Autophagic mechanisms are related to the TSC1/2 proteins, which up-regulate this pathway, which becomes inhibited upon mTORC1 hyperactivation (Martina, Chen et al. 2012, Efeyan, Zoncu et al. 2013).

We did not find significant enrichment in gene sets for up-regulated genes independently on the presence of serum. However, up-regulated gene sets in DMEMplus group did relate to cell morphogenesis. This transcriptional trait for cell morphology of $1 \mathrm{C} 2 \mathrm{TSC}^{-1}$ are in agreement with previously reports, in which overexpression of the TSC1 gene product hamartin results in changes of cell morphology (Benvenuto, Li et al. 2000).

Finally, when only the $1 \mathrm{C} 2$ cell line was considered for global RNA analyses between DMEMminus and DMEMplus groups, more than 50 down-regulated genes have been detected. Similarly to up-regulated genes on DMEMminus group, response to topologically incorrect protein is both down- and up-regulated, which means that incorrect protein folding is an intrinsic characteristic of the $1 \mathrm{CL}^{\mathrm{TSC}} \mathrm{TS}^{-}$cell line. Interestingly, response to endoplasmic reticulum (ER) stress is down-regulated. The ER is the cellular compartment where synthesis of proteins for the secretory pathway takes place. The ER stress response has been observed in mTORC1 activation in TSC2-null Elt3 cells via c-MYC translation (Babcock, Nguyen et al. 2013). mTORC1 hyperactivation increases the basal level of ER stress via an accumulation of unfolded proteins (Clarke, Chambers et al. 2014, Johnson, Dunlop et al. 2018). Taking together, a stress on this cellular compartment due to mTORC1 hyperactivation may cause an accumulation of unfolded proteins leading to a negative feedback and down regulate genes related to post-transcritptional steps, as observed for $1 \mathrm{C} 2$ cell line. It is worth to note that ER stress can develop in TSC-associated cells due to mTORC1-driven protein translation. mTORC1 inhibition of ER stress by RAD001 suppressed angiomyolipoma cell proliferation in a cytostatic manner (Siroky, Yin et al. 2012). Finally, protein folding and response to temperature stress are up-regulated in the $1 C 2$ cell line, consistent with the role of TSC1 protein as a heat-shock co-chaperone protein (Woodford, Sager et al. 2017). 


\section{Conclusions}

In this study, we employed six cell lines derived from HEK293T cell line by CRISPR-Cas9 editing of the TSC1 and/or TSC2 genes. Western blotting analyses have shown no protein expressed by the respective inactivated gene, and mTORC1 hyperactivation. Serum starvation for 16 hours did not affect the phosphorylation status of Akt residues $\mathrm{Thr}^{308}$ and $\mathrm{Ser}^{473}$, as well as the overall protein levels of upstream AKT protein or downstream S6 protein. TSC2 levels were reduced in $\mathrm{TSC1}^{-/}$cells relative to the wild-type cell line HEK293T; likewise, the protein levels of TSC1 decreased in $\mathrm{TSC2}^{-\%}$ cells. The $\mathrm{TSC}^{-/}$cell line $1 \mathrm{C} 2$ was selected for RNA-Seq analyses as it harbored limited TSC1 indel variants produced by CRISP/Cas editing, thus not impacting its mRNA length. It also consistently presented reduction of TSC2 mRNA and protein amount. Upon serum withdrawal, $1 \mathrm{C2}$ cells had significant alterations of mRNA amount of genes involved in protein folding, which clustered in two distinct groups of either up- or down-regulated mRNA levels. The gene IDs in each group are consistent with the role of TSC1 as co-chaperone of heat-shock proteins. 


\section{References}

Abeysinghe, S. S., N. Chuzhanova, M. Krawczak, E. V. Ball and D. N. Cooper (2003). "Translocation and gross deletion breakpoints in human inherited disease and cancer I: Nucleotide composition and recombination-associated motifs." Hum Mutat 22(3): 229-244.

Abo-Dalo, B., K. Kutsche, V. Mautner and L. Kluwe (2010). "Large intragenic deletions of the NF2 gene: breakpoints and associated phenotypes." Genes Chromosomes Cancer 49(2): 171-175.

Adriaensen, M. E., C. M. Schaefer-Prokop, T. Stijnen, D. A. Duyndam, B. A. Zonnenberg and M. Prokop (2009). "Prevalence of subependymal giant cell tumors in patients with tuberous sclerosis and a review of the literature." Eur J Neurol 16(6): 691-696.

Adzhubei, I. A., S. Schmidt, L. Peshkin, V. E. Ramensky, A. Gerasimova, P. Bork, A. S. Kondrashov and S. R. Sunyaev (2010). "A method and server for predicting damaging missense mutations." Nat Methods 7(4): 248-249.

Ambar, G. and S. Chiavegatto (2009). "Anabolic-androgenic steroid treatment induces behavioral disinhibition and downregulation of serotonin receptor messenger RNA in the prefrontal cortex and amygdala of male mice." Genes Brain Behav 8(2): 161-173.

Ashfaq, R., A. G. Weinberg and J. Albores-Saavedra (1993). "Renal angiomyolipomas and HMB-45 reactivity." Cancer 71(10): 3091-3097.

Astrinidis, A., L. Khare, T. Carsillo, T. Smolarek, K. S. Au, H. Northrup and E. P. Henske (2000). "Mutational analysis of the tuberous sclerosis gene TSC2 in patients with pulmonary lymphangioleiomyomatosis." J Med Genet 37(1): 55-57.

Astrinidis, A., W. Senapedis and E. P. Henske (2006). "Hamartin, the tuberous sclerosis complex 1 gene product, interacts with polo-like kinase 1 in a phosphorylation-dependent manner." Hum Mol Genet 15(2): 287-297.

Au, K. S., J. A. Rodriguez, J. L. Finch, K. A. Volcik, E. S. Roach, M. R. Delgado, E. Rodriguez, Jr. and H. Northrup (1998). "Germ-line mutational analysis of the TSC2 gene in 90 tuberous-sclerosis patients." Am J Hum Genet 62(2): 286-294.

Au, K. S., A. T. Williams, E. S. Roach, L. Batchelor, S. P. Sparagana, M. R. Delgado, J. W. Wheless, J. E. Baumgartner, B. B. Roa, C. M. Wilson, T. K. Smith-Knuppel, M. Y. Cheung, V. H. Whittemore, T. M. King and H. Northrup (2007). "Genotype/phenotype correlation in 325 individuals referred for a diagnosis of tuberous sclerosis complex in the United States." Genet Med 9(2): 88-100.

Babcock, J. T., H. B. Nguyen, Y. He, J. W. Hendricks, R. C. Wek and L. A. Quilliam (2013). "Mammalian target of rapamycin complex 1 (mTORC1) enhances bortezomib-induced death in tuberous sclerosis complex (TSC)-null cells by a c-MYC-dependent induction of the unfolded protein response." J Biol Chem 288(22): 15687-15698.

Bar-Peled, L., L. D. Schweitzer, R. Zoncu and D. M. Sabatini (2012). "Ragulator is a GEF for the rag GTPases that signal amino acid levels to mTORC1." Cell 150(6): 1196-1208.

Bastos, A. P. and L. F. Onuchic (2011). "Molecular and cellular pathogenesis of autosomal dominant polycystic kidney disease." Braz J Med Biol Res 44(7): 606-617.

Beauchamp, R. L., A. Banwell, P. McNamara, M. Jacobsen, E. Higgins, H. Northrup, P. Short, K. Sims, L. Ozelius and V. Ramesh (1998). "Exon scanning of the entire TSC2 gene for germline mutations in 40 unrelated patients with tuberous sclerosis." Hum Mutat 12(6): 408416.

Benvenuto, G., S. Li, S. J. Brown, R. Braverman, W. C. Vass, J. P. Cheadle, D. J. Halley, J. R. Sampson, R. Wienecke and J. E. DeClue (2000). "The tuberous sclerosis-1 (TSC1) gene product hamartin suppresses cell growth and augments the expression of the TSC2 product tuberin by inhibiting its ubiquitination." Oncogene 19(54): 6306-6316.

Boronat, S., I. Barber and E. A. Thiele (2017). "Sclerotic bone lesions in tuberous sclerosis complex: A genotype-phenotype study." Am J Med Genet A. 
Bourneville, D. M. (1880). "Sclérose tubéreuse des circonvolutions cérébrales." Arch Neurol 1: 11.

Brown, M. A., K. Foreman, J. Harriss, C. Das, L. Zhu, M. Edwards, S. Shaaban and H. Tucker (2015). "C-terminal domain of SMYD3 serves as a unique HSP90-regulated motif in oncogenesis." Oncotarget 6(6): 4005-4019.

Buccoliero, A. M., A. Franchi, F. Castiglione, C. F. Gheri, F. Mussa, F. Giordano, L. Genitori and G. L. Taddei (2009). "Subependymal giant cell astrocytoma (SEGA): Is it an astrocytoma? Morphological, immunohistochemical and ultrastructural study." Neuropathology 29(1): 25-30.

Bukau, B. and A. L. Horwich (1998). "The Hsp70 and Hsp60 chaperone machines." Cell 92(3): 351-366.

Byfield, M. P., J. T. Murray and J. M. Backer (2005). "hVps34 is a nutrient-regulated lipid kinase required for activation of p70 S6 kinase." J Biol Chem 280(38): 33076-33082.

Calderwood, S. K. and A. Murshid (2017). "Molecular Chaperone Accumulation in Cancer and Decrease in Alzheimer's Disease: The Potential Roles of HSF1." Front Neurosci 11: 192.

Camposano, S. E., E. Greenberg, D. J. Kwiatkowski and E. A. Thiele (2009). "Distinct clinical characteristics of tuberous sclerosis complex patients with no mutation identified." Ann Hum Genet 73(2): 141-146.

Carlisle, C., K. Prill and D. Pilgrim (2017). "Chaperones and the Proteasome System: Regulating the Construction and Demolition of Striated Muscle." Int J Mol Sci 19(1).

Carsillo, T., A. Astrinidis and E. P. Henske (2000). "Mutations in the tuberous sclerosis complex gene TSC2 are a cause of sporadic pulmonary lymphangioleiomyomatosis." Proc Natl Acad Sci U S A 97(11): 6085-6090.

Castro, M., C. W. Shepherd, M. R. Gomez, J. T. Lie and J. H. Ryu (1995). "Pulmonary tuberous sclerosis." Chest 107(1): $189-195$.

Caylor, R. C., L. Grote, I. Thiffault, E. G. Farrow, L. Willig, S. Soden, S. M. Amudhavalli, A. J. Nopper, K. A. Horii, E. Fleming, J. Jenkins, H. Welsh, M. Ilyas, K. Engleman, A. Abdelmoity and C. J. Saunders (2018). "Incidental diagnosis of tuberous sclerosis complex by exome sequencing in three families with subclinical findings." Neurogenetics 19(3): 205-213.

Chiron, C., C. Dumas, I. Jambaqué, J. Mumford and O. Dulac (1997). "Randomized trial comparing vigabatrin and hydrocortisone in infantile spasms due to tuberous sclerosis." Epilepsy Research 26(2): 389-395.

Choi, Y. and A. P. Chan (2015). "PROVEAN web server: a tool to predict the functional effect of amino acid substitutions and indels." Bioinformatics 31(16): 2745-2747.

Chong-Kopera, H., K. Inoki, Y. Li, T. Zhu, F. R. Garcia-Gonzalo, J. L. Rosa and K. L. Guan (2006). "TSC1 stabilizes TSC2 by inhibiting the interaction between TSC2 and the HERC1 ubiquitin ligase." J Biol Chem 281(13): 8313-8316.

Chopra, M., J. A. Lawson, M. Wilson, S. E. Kennedy, P. Taylor, M. F. Buckley, O. Wargon, G. Parasivam, C. Camphausen, D. Yates and D. Mowat (2011). "An Australian tuberous sclerosis cohort: are surveillance guidelines being met?" J Paediatr Child Health 47(10): 711-716.

Chu-Shore, C. J., P. Major, S. Camposano, D. Muzykewicz and E. A. Thiele (2010). "The natural history of epilepsy in tuberous sclerosis complex." Epilepsia 51(7): 1236-1241.

Clarke, H. J., J. E. Chambers, E. Liniker and S. J. Marciniak (2014). "Endoplasmic reticulum stress in malignancy." Cancer Cell 25(5): 563573.

Connor, J. M., J. B. Stephenson and M. D. Hadley (1986). "Non-penetrance in tuberous sclerosis." Lancet 2(8518): 1275. 
Consortium, T. E. C. T. S. (1993). "Identification and characterization of the tuberous sclerosis gene on chromosome 16." Cell 75(7): 1305-1315.

Consugar, M. B., W. C. Wong, P. A. Lundquist, S. Rossetti, V. J. Kubly, D. L. Walker, L. J. Rangel, R. Aspinwall, W. P. Niaudet, S. Ozen, A. David, M. Velinov, E. J. Bergstralh, K. T. Bae, A. B. Chapman, L. M. Guay-Woodford, J. J. Grantham, V. E. Torres, J. R. Sampson, B. D. Dawson, P. C. Harris and C. Consortium (2008). "Characterization of large rearrangements in autosomal dominant polycystic kidney disease and the PKD1/TSC2 contiguous gene syndrome." Kidney Int 74(11): 1468-1479.

Cooper, J. R. (1971). "Brain tumors in hereditary multiple system hamartomatosis (tuberous sclerosis)." J Neurosurg 34(2 Pt 1): 194-202.

Crino, P. B. (2013). "Evolving neurobiology of tuberous sclerosis complex." Acta Neuropathol 125(3): 317-332.

Crundwell, M. (2004). "Pathology and genetics of tumours of the urinary system and male genital organs." BJU International 94(4): 675675.

Curatolo, P., S. Seri, M. Verdecchia and R. Bombardieri (2001). "Infantile spasms in tuberous sclerosis complex." Brain Dev 23(7): 502507.

Dabora, S. L., D. N. Franz, S. Ashwal, A. Sagalowsky, F. J. DiMario, Jr., D. Miles, D. Cutler, D. Krueger, R. N. Uppot, R. Rabenou, S. Camposano, J. Paolini, F. Fennessy, N. Lee, C. Woodrum, J. Manola, J. Garber and E. A. Thiele (2011). "Multicenter phase 2 trial of sirolimus for tuberous sclerosis: kidney angiomyolipomas and other tumors regress and VEGF- D levels decrease." PLoS One 6(9): e23379.

Dabora, S. L., S. Jozwiak, D. N. Franz, P. S. Roberts, A. Nieto, J. Chung, Y. S. Choy, M. P. Reeve, E. Thiele, J. C. Egelhoff, J. Kasprzyk-Obara, D. Domanska-Pakiela and D. J. Kwiatkowski (2001). "Mutational analysis in a cohort of 224 tuberous sclerosis patients indicates increased severity of TSC2, compared with TSC1, disease in multiple organs." Am J Hum Genet 68(1): 64-80.

Dan, H. C., M. Sun, L. Yang, R. I. Feldman, X. M. Sui, C. C. Ou, M. Nellist, R. S. Yeung, D. J. Halley, S. V. Nicosia, W. J. Pledger and J. Q. Cheng (2002). "Phosphatidylinositol 3-kinase/Akt pathway regulates tuberous sclerosis tumor suppressor complex by phosphorylation of tuberin." J Biol Chem 277(38): 35364-35370.

Davidson, M., H. Yoshidome, E. Stenroos and W. G. Johnson (1991). "Neuron-like cells in culture of tuberous sclerosis tissue." Ann N Y Acad Sci 615: 196-210.

Demetriades, C., N. Doumpas and A. A. Teleman (2014). "Regulation of TORC1 in response to amino acid starvation via lysosomal recruitment of TSC2." Cell 156(4): 786-799.

Desmet, F. O., D. Hamroun, M. Lalande, G. Collod-Beroud, M. Claustres and C. Beroud (2009). "Human Splicing Finder: an online bioinformatics tool to predict splicing signals." Nucleic Acids Res 37(9): e67.

Dufner-Almeida, L. (2014). Estudo mutacional em pacientes com o complexo da esclerose tuberosa. Dissertação de Mesteado Dissertação de Mestrado, Universidade de São Paulo.

Eble, J. N. (1998). "Angiomyolipoma of kidney." Semin Diagn Pathol 15(1): 21-40.

Efeyan, A., R. Zoncu, S. Chang, I. Gumper, H. Snitkin, R. L. Wolfson, O. Kirak, D. D. Sabatini and D. M. Sabatini (2013). "Regulation of mTORC1 by the Rag GTPases is necessary for neonatal autophagy and survival." Nature 493(7434): 679-683.

Fagerberg, L., B. M. Hallstrom, P. Oksvold, C. Kampf, D. Djureinovic, J. Odeberg, M. Habuka, S. Tahmasebpoor, A. Danielsson, K. Edlund, A. Asplund, E. Sjostedt, E. Lundberg, C. A. Szigyarto, M. Skogs, J. O. Takanen, H. Berling, H. Tegel, J. Mulder, P. Nilsson, J. M. Schwenk, C. Lindskog, F. Danielsson, A. Mardinoglu, A. Sivertsson, K. von Feilitzen, M. Forsberg, M. Zwahlen, I. Olsson, S. Navani, M. Huss, J. Nielsen, F. Ponten and M. Uhlen (2014). "Analysis of the human tissue-specific expression by genome-wide integration of transcriptomics and antibody-based proteomics." Mol Cell Proteomics 13(2): 397-406. 
Fernandez-Flores, A. (2011). "Perivascular migration: a clue to the histogenesis of PEComas?" Am J Dermatopathol 33(5): 528-529.

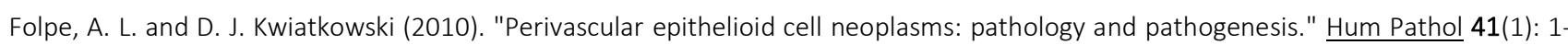
15.

Franz, D. N., J. J. Bissler and F. X. McCormack (2010). "Tuberous sclerosis complex: neurological, renal and pulmonary manifestations." Neuropediatrics 41(5): 199-208.

Freeman, P. J., R. K. Hart, L. J. Gretton, A. J. Brookes and R. Dalgleish (2018). "VariantValidator: Accurate validation, mapping, and formatting of sequence variation descriptions." Hum Mutat 39(1): 61-68.

Frey, U. H., H. S. Bachmann, J. Peters and W. Siffert (2008). "PCR-amplification of GC-rich regions: 'slowdown PCR'." Nat Protoc 3(8): 1312-1317.

Gai, Z., W. Chu, W. Deng, W. Li, H. Li, A. He, M. Nellist and G. Wu (2016). "Structure of the TBC1D7-TSC1 complex reveals that TBC1D7 stabilizes dimerization of the TSC1 C-terminal coiled coil region." J Mol Cell Biol.

Garami, A., F. J. T. Zwartkruis, T. Nobukuni, M. Joaquin, M. Roccio, H. Stocker, S. C. Kozma, E. Hafen, J. L. Bos and G. Thomas (2003). "Insulin Activation of Rheb, a Mediator of mTOR/S6K/4E-BP Signaling, Is Inhibited by TSC1 and 2." Molecular Cell 11(6): 1457-1466.

Gerstein, M. B., C. Bruce, J. S. Rozowsky, D. Zheng, J. Du, J. O. Korbel, O. Emanuelsson, Z. D. Zhang, S. Weissman and M. Sny der (2007). "What is a gene, post-ENCODE? History and updated definition." Genome Res 17(6): 669-681.

Gomez, M., J. Sampson and V. Holtes-Whittemore (1999). "Tuberous Sclerosis Complex." Oxford University Press 3rd edition.

Goncharova, E., D. Goncharov, D. Noonan and V. P. Krymskaya (2004). "TSC2 modulates actin cytoskeleton and focal adhesion through TSC1-binding domain and the Rac1 GTPase." J Cell Biol 167(6): 1171-1182.

Goncharova, E. A., D. A. Goncharov, A. Eszterhas, D. S. Hunter, M. K. Glassberg, R. S. Yeung, C. L. Walker, D. Noonan, D. J. Kwiatkowski, M. M. Chou, R. A. Panettieri, Jr. and V. P. Krymskaya (2002). "Tuberin regulates p70 S6 kinase activation and ribosomal protein S6 phosphorylation. A role for the TSC2 tumor suppressor gene in pulmonary lymphangioleiomyomatosis (LAM)." J Biol Chem 277(34): 30958-30967.

Goodman, M., S. H. Lamm, A. Engel, C. W. Shepherd, O. W. Houser and M. R. Gomez (1997). "Cortical tuber count: a biomarker indicating neurologic severity of tuberous sclerosis complex." J Child Neurol 12(2): 85-90.

Graham, F. L., J. Smiley, W. C. Russell and R. Nairn (1977). "Characteristics of a human cell line transformed by DNA from human adenovirus type 5." J Gen Virol 36(1): 59-74.

Grajkowska, W., K. Kotulska, E. Jurkiewicz and E. Matyja (2010). "Brain lesions in tuberous sclerosis complex. Review." Folia Neuropathol 48(3): 139-149.

Gu, S., B. Yuan, I. M. Campbell, C. R. Beck, C. M. Carvalho, S. C. Nagamani, A. Erez, A. Patel, C. A. Bacino, C. A. Shaw, P. Stankiewicz, S. W. Cheung, W. Bi and J. R. Lupski (2015). "Alu-mediated diverse and complex pathogenic copy-number variants within human chromosome 17 at p13.3." Hum Mol Genet 24(14): 4061-4077.

Hancock, E. and J. Osborne (1998). "Treatment of infantile spasms with high-dose oral prednisolone." Dev Med Child Neurol 40(7): 500

Hasbani, D. M. and P. B. Crino (2018). "Tuberous sclerosis complex." Handb Clin Neurol 148: 813-822.

Hayashi, T., T. Kumasaka, K. Mitani, T. Yao, K. Suda and K. Seyama (2010). "Loss of heterozygosity on tuberous sclerosis complex genes in multifocal micronodular pneumocyte hyperplasia." Mod Pathol 23(9): 1251-1260. 
Holmes, G. L., C. E. Stafstrom and G. Tuberous Sclerosis Study (2007). "Tuberous sclerosis complex and epilepsy: recent developments and future challenges." Epilepsia 48(4): 617-630.

Hoogeveen-Westerveld, M., R. Ekong, S. Povey, I. Karbassi, S. D. Batish, J. T. den Dunnen, A. van Eeghen, E. Thiele, K. Mayer, K. Dies, L. Wen, C. Thompson, S. P. Sparagana, P. Davies, C. Aalfs, A. van den Ouweland, D. Halley and M. Nellist (2012). "Functional assessment of TSC1 missense variants identified in individuals with tuberous sclerosis complex." Hum Mutat 33(3): 476-479.

Hoogeveen-Westerveld, M., R. Ekong, S. Povey, K. Mayer, N. Lannoy, F. Elmslie, M. Bebin, K. Dies, C. Thompson, S. P. Sparagana, P. Davies, A. M. van Eeghen, E. A. Thiele, A. van den Ouweland, D. Halley and M. Nellist (2013). "Functional assessment of TSC2 variants identified in individuals with tuberous sclerosis complex." Hum Mutat 34(1): 167-175.

Hoogeveen-Westerveld, M., L. van Unen, A. van den Ouweland, D. Halley, A. Hoogeveen and M. Nellist (2012). "The TSC1-TSC2 complex consists of multiple TSC1 and TSC2 subunits." BMC Biochem 13: 18.

Hoogeveen-Westerveld, M., M. Wentink, D. van den Heuvel, M. Mozaffari, R. Ekong, S. Povey, J. T. den Dunnen, K. Metcalfe, S. Vallee, S. Krueger, J. Bergoffen, V. Shashi, F. Elmslie, D. Kwiatkowski, J. Sampson, C. Vidales, J. Dzarir, J. Garcia-Planells, K. Dies, A. Maat-Kievit, A. van den Ouweland, D. Halley and M. Nellist (2011). "Functional assessment of variants in the TSC1 and TSC2 genes identified in individuals with Tuberous Sclerosis Complex." Hum Mutat 32(4): 424-435.

Hornbeck, P. V., B. Zhang, B. Murray, J. M. Kornhauser, V. Latham and E. Skrzypek (2015). "PhosphoSitePlus, 2014: mutations, PTMs and recalibrations." Nucleic Acids Res 43(Database issue): D512-520.

Hosoyaa, M., H. Naitoa and K. Nihei (1999). "Neurological prognosis correlated with variations over time in the number of subependymal nodules in tuberous sclerosis." Brain \& Development 21(8): 4.

Houser, O. W. and M. R. Gomez (1992). "CT and MR imaging of intracranial tuberous sclerosis." J Dermatol 19(11): 904-908.

Humphrey, A., J. Williams, E. Pinto and P. F. Bolton (2004). "A prospective longitudinal study of early cognitive development in tuberous sclerosis - a clinic based study." Eur Child Adolesc Psychiatry 13(3): 159-165.

Hung, C. C., Y. N. Su, S. C. Chien, H. H. Liou, C. C. Chen, P. C. Chen, C. J. Hsieh, C. P. Chen, W. T. Lee, W. L. Lin and C. N. Lee (2006). "Molecular and clinical analyses of 84 patients with tuberous sclerosis complex." BMC Med Genet 7: 72.

Huttenlocher, P. R. and R. L. Wollmann (1991). "Cellular neuropathology of tuberous sclerosis." Ann N Y Acad Sci 615: 140-148.

Inoki, K., Y. Li, T. Xu and K. L. Guan (2003). "Rheb GTPase is a direct target of TSC2 GAP activity and regulates mTOR signaling." Genes Dev 17(15): 1829-1834.

Inoue, H., T. Uyama, T. Suzuki, M. Kazami, O. Hino, T. Kobayashi, K. Kobayashi, T. Tadokoro and Y. Yamamoto (2010). "Phosphorylated hamartin-Hsp70 complex regulates apoptosis via mitochondrial localization." Biochem Biophys Res Commun 391(1): 1148-1153.

Ismail, N. F., A. Q. Rani, N. M. Nik Abdul Malik, C. Boon Hock, S. N. Mohd Azlan, S. Abdul Razak, W. T. Keng, L. H. Ngu, A. R. Silawati, N. A. Yahya, N. Mohd Yusoff, T. H. Sasongko and Z. A. Zabidi-Hussin (2017). "Combination of Multiple Ligation-Dependent Probe Amplification and Illumina MiSeq Amplicon Sequencing for TSC1/TSC2 Gene Analyses in Patients with Tuberous Sclerosis Complex." J Mol Diagn 19(2) 265-276.

Jansen, F. E., O. van Nieuwenhuizen and A. C. van Huffelen (2004). "Tuberous sclerosis complex and its founders." J Neurol Neurosurg Psychiatry 75(5): 770.

Johnson, C. E., E. A. Dunlop, S. Seifan, H. D. McCann, T. Hay, G. J. Parfitt, A. T. Jones, P. J. Giles, M. H. Shen, J. R. Sampson, R. J. Errington, D. M. Davies and A. R. Tee (2018). "Loss of tuberous sclerosis complex 2 sensitizes tumors to nelfinavir-bortezomib therapy to intensify endoplasmic reticulum stress-induced cell death." Oncogene 37(45): 5913-5925. 
Johnson, W. G., H. Yoshidome, E. S. Stenroos and M. M. Davidson (1991). "Origin of the neuron-like cells in tuberous sclerosis tissues." Ann N Y Acad Sci 615: 211-219.

Joinson, C., F. J. O'Callaghan, J. P. Osborne, C. Martyn, T. Harris and P. F. Bolton (2003). "Learning disability and epilepsy in an epidemiological sample of individuals with tuberous sclerosis complex." Psychol Med 33(2): 335-344.

Jones, A. C., C. E. Daniells, R. G. Snell, M. Tachataki, S. A. Idziaszczyk, M. Krawczak, J. R. Sampson and J. P. Cheadle (1997). "Molecular Genetic and Phenotypic Analysis Reveals Differences between TSC1 and TSC2 Associated Familial and Sporadic Tuberous Sclerosis." Human Molecular Genetics 6(12): 2155-2161

Jones, A. C., M. M. Shyamsundar, M. W. Thomas, J. Maynard, S. Idziaszczyk, S. Tomkins, J. R. Sampson and J. P. Cheadle (1999). "Comprehensive mutation analysis of TSC1 and TSC2-and phenotypic correlations in 150 families with tuberous sclerosis." Am J Hum Genet 64(5): 1305-1315.

Jozwiak, J., S. Jozwiak and P. Skopinski (2005). "Immunohistochemical and microscopic studies on giant cells in tuberous sclerosis." $\underline{\text { Histol }}$ Histopathol 20(4): 1321-1326.

Kim, M. J., S. I. Cho, J. H. Chae, B. C. Lim, J. S. Lee, S. J. Lee, S. H. Seo, H. Park, A. Cho, S. Y. Kim, J. Y. Kim, S. S. Park and M. W. Seong (2016). "Pitfalls of Multiple Ligation-Dependent Probe Amplifications in Detecting DMD Exon Deletions or Duplications." J Mol Diagn 18(2): 253 259.

Knudson, A. G., Jr. (1971). "Mutation and cancer: statistical study of retinoblastoma." Proc Natl Acad Sci U S A 68(4): 820-823.

Kozlowski, P., P. Roberts, S. Dabora, D. Franz, J. Bissler, H. Northrup, K. S. Au, R. Lazarus, D. Domanska-Pakiela, K. Kotulska, S. Jozwiak and D. J. Kwiatkowski (2007). "Identification of 54 large deletions/duplications in TSC1 and TSC2 using MLPA, and genotype-phenotype correlations." Hum Genet 121(3-4): 389-400.

Krueger, D. A., H. Northrup and G. International Tuberous Sclerosis Complex Consensus (2013). "Tuberous sclerosis complex surveillance and management: recommendations of the 2012 International Tuberous Sclerosis Complex Consensus Conference." Pediatr Neurol 49(4): 255-265.

Kumar, P., S. Henikoff and P. C. Ng (2009). "Predicting the effects of coding non-synonymous variants on protein function using the SIFT algorithm." Nat Protoc 4(7): 1073-1081.

Kwiatkowski, D. J. (1994). "Tuberous Sclerosis." Archives of Dermatology 130(3).

Kwiatkowski, D. J. and B. D. Manning (2005). "Tuberous sclerosis: a GAP at the crossroads of multiple signaling pathways." Genet 14 Spec No. 2: R251-258.

Kwiatkowski, D. J., M. R. Palmer, S. Jozwiak, J. Bissler, D. Franz, S. Segal, D. Chen and J. R. Sampson (2015). "Response to everolimus is seen in TSC-associated SEGAs and angiomyolipomas independent of mutation type and site in TSC1 and TSC2." Eur J Hum Genet 23(12): 1665-1672.

Lamb, R. F., C. Roy, T. J. Diefenbach, H. V. Vinters, M. W. Johnson, D. G. Jay and A. Hall (2000). "The TSC1 tumour suppressor hamartin regulates cell adhesion through ERM proteins and the GTPase Rho." Nat Cell Biol 2(5): 281-287.

Li, H. and R. Durbin (2009). "Fast and accurate short read alignment with Burrows-Wheeler Transform. Bioinformatics." Bioinformatics 25: 7.

Li, W., L. H. Zhou, B. D. Gao, L. Y. Li, C. G. Zhong, F. Gong, H. M. Xiao, T. Song and G. X. Lu (2011). "[Mutation screening and prenatal diagnosis of tuberous sclerosis complex]." Zhonghua Yi Xue Yi Chuan Xue Za Zhi 28(4): 361-366. 
Lilyestrom, W., M. G. Klein, R. G. Zhang, A. Joachimiak and X. J. S. Chen (2006). "Crystal structure of SV40 large T-antigen bound to p53: interplay between a viral oncoprotein and a cellular tumor suppressor." Genes \& Development 20(17): 2373-2382.

Lin, Y. C., M. Boone, L. Meuris, I. Lemmens, N. Van Roy, A. Soete, J. Reumers, M. Moisse, S. Plaisance, R. Drmanac, J. Chen, F. Speleman, D. Lambrechts, Y. Van de Peer, J. Tavernier and N. Callewaert (2014). "Genome dynamics of the human embryonic kidney 293 lineage in response to cell biology manipulations." Nat Commun 5: 4767.

Lotz, G. P., H. Lin, A. Harst and W. M. Obermann (2003). "Aha1 binds to the middle domain of Hsp90, contributes to client protein activation, and stimulates the ATPase activity of the molecular chaperone." J Biol Chem 278(19): 17228-17235.

Love, M. I., W. Huber and S. Anders (2014). "Moderated estimation of fold change and dispersion for RNA-seq data with DESeq2." Genome Biol 15(12): 550

Maheshwar, M. M., J. P. Cheadle, A. C. Jones, J. Myring, A. E. Fryer, P. C. Harris and J. R. Sampson (1997). "The GAP-Related Domain of Tuberin, the Product of the TSC2 Gene, is a Target for Missense Mutations in Tuberous Sclerosis." Human Molecular Genetics 6(11) 1991-1996.

Manning, B. D., A. R. Tee, M. N. Logsdon, J. Blenis and L. C. Cantley (2002). "Identification of the Tuberous Sclerosis Complex-2 Tumor Suppressor Gene Product Tuberin as a Target of the Phosphoinositide 3-Kinase/Akt Pathway." Molecular Cell 10(1): 151-162.

Martignoni, G., F. Bonetti, M. Pea, R. Tardanico, M. Brunelli and J. N. Eble (2002). "Renal disease in adults with TSC2/PKD1 contiguous gene syndrome." Am J Surg Pathol 26(2): 198-205.

Martignoni, G., M. Pea, D. Reghellin, G. Zamboni and F. Bonetti (2008). "PEComas: the past, the present and the future." Virchows Arch 452(2): 119-132.

Martina, J. A., Y. Chen, M. Gucek and R. Puertollano (2012). "MTORC1 functions as a transcriptional regulator of autophagy by preventing nuclear transport of TFEB." Autophagy 8(6): 903-914.

Maruyama, H., K. Seyama, J. Sobajima, K. Kitamura, T. Sobajima, T. Fukuda, K. Hamada, M. Tsutsumi, O. Hino and Y. Konishi (2001). "Multifocal micronodular pneumocyte hyperplasia and lymphangioleiomyomatosis in tuberous sclerosis with a TSC2 gene." Mod Pathol 14(6): 609-614.

McCall, T., S. S. Chin, K. L. Salzman and D. W. Fults (2006). "Tuberous sclerosis: a syndrome of incomplete tumor suppression." Neurosurg Focus 20(1): E3.

McKenna, A., M. Hanna, E. Banks, A. Sivachenko, K. Cibulskis, A. Kernytsky, K. Garimella, D. Altshuler, S. Gabriel, M. Daly and M. A. DePristo (2010). "The Genome Analysis Toolkit: a MapReduce framework for analyzing next-generation DNA sequencing data." Genome Res 20(9): 1297-1303.

Mizuguchi, M. and S. Takashima (2001). "Neuropathology of tuberous sclerosis." Brain Dev 23(7): 508-515.

Moavero, R., M. Pinci, R. Bombardieri and P. Curatolo (2011). "The management of subependymal giant cell tumors in tuberous sclerosis: a clinician's perspective." Childs Nerv Syst 27(8): 1203-1210.

Moss, J., N. A. Avila, P. M. Barnes, R. A. Litzenberger, J. Bechtle, P. G. Brooks, C. J. Hedin, S. Hunsberger and A. S. Kristof (2001). "Prevalence and clinical characteristics of lymphangioleiomyomatosis (LAM) in patients with tuberous sclerosis complex." Am J Respir Crit Care Med 164(4): 669-671.

Muir, T. E., K. O. Leslie, H. Popper, M. Kitaichi, E. Gagné, J. K. Emelin, H. V. Vinters and T. V. Colby (1998). "Micronodular pneumocyte hyperplasia." Am J Surg Pathol 22(4): 8. 
Nellist, M., R. W. Brouwer, C. E. Kockx, M. van Veghel-Plandsoen, C. Withagen-Hermans, L. Prins-Bakker, M. Hoogeveen-Westerveld, A. Mrsic, M. M. van den Berg, A. E. Koopmans, M. C. de Wit, F. E. Jansen, A. J. Maat-Kievit, A. van den Ouweland, D. Halley, A. de Klein and I. W. F. van (2015). "Targeted Next Generation Sequencing reveals previously unidentified TSC1 and TSC2 mutations." BMC Med Genet 16: 10.

Nellist, M., O. Sancak, M. A. Goedbloed, C. Rohe, D. van Netten, K. Mayer, A. Tucker-Williams, A. M. van den Ouweland and D. J. Halley (2005). "Distinct effects of single amino-acid changes to tuberin on the function of the tuberin-hamartin complex." Eur J Hum Genet 13(1): 59-68

Nellist, M., D. van den Heuvel, D. Schluep, C. Exalto, M. Goedbloed, A. Maat-Kievit, T. van Essen, K. van Spaendonck-Zwarts, F. Jansen, P. Helderman, G. Bartalini, O. Vierimaa, M. Penttinen, J. van den Ende, A. van den Ouweland and D. Halley (2009). "Missense mutations to the TSC1 gene cause tuberous sclerosis complex." Eur J Hum Genet 17(3): 319-328.

Nellist, M., M. A. van Slegtenhorst, M. Goedbloed, A. M. van den Ouweland, D. J. Halley and P. van der Sluijs (1999). "Characterization of the cytosolic tuberin-hamartin complex. Tuberin is a cytosolic chaperone for hamartin." J Biol Chem 274(50): 35647-35652.

Ng, P. C. and S. Henikoff (2003). "SIFT: predicting amino acid changes that affect protein function." Nucleic Acids Research 31(13): 3812 3814.

Ng, P. C. and S. Henikoff (2006). "Predicting the effects of amino acid substitutions on protein function." Annu Rev Genomics Hum Genet 7: 61-80.

Nicholson, K. M. and N. G. Anderson (2002). "The protein kinase B/Akt signalling pathway in human malignancy." Cellular Signalling 14(5): 381-395.

Niida, Y., N. Lawrence-Smith, A. Banwell, E. Hammer, J. Lewis, R. L. Beauchamp, K. Sims, V. Ramesh and L. Ozelius (1999). "Analysis of bothTSC1 andTSC2 for germline mutations in 126 unrelated patients with tuberous sclerosis." Human Mutation 14(5): $412-422$.

Niida, Y., A. O. Stemmer-Rachamimov, M. Logrip, D. Tapon, R. Perez, D. J. Kwiatkowski, K. Sims, M. MacCollin, D. N. Louis and V. Ramesh (2001). "Survey of somatic mutations in tuberous sclerosis complex (TSC) hamartomas suggests different genetic mechanisms for pathogenesis of TSC lesions." Am J Hum Genet 69(3): 493-503.

Niida, Y., A. Wakisaka, T. Tsuji, H. Yamada, M. Kuroda, Y. Mitani, A. Okumura and A. Yokoi (2013). "Mutational analysis of TSC1 and TSC2 in Japanese patients with tuberous sclerosis complex revealed higher incidence of TSC1 patients than previously reported." $\underline{\mathrm{J} \text { Hum Genet }}$ 58(4): 216-225.

Nishio, S., T. Morioka, S. Suzuki, R. Kira, F. Mihara and M. Fukui (2001). "Subependymal giant cell astrocytoma: clinical and neuroimaging features of four cases." J Clin Neurosci 8(1): 31-34.

Nobukuni, T., M. Joaquin, M. Roccio, S. G. Dann, S. Y. Kim, P. Gulati, M. P. Byfield, J. M. Backer, F. Natt, J. L. Bos, F. J. Zwartkruis and G. Thomas (2005). "Amino acids mediate mTOR/raptor signaling through activation of class 3 phosphatidylinositol 3OH-kinase." Proc Natl Acad Sci U S A 102(40): 14238-14243.

Northrup, H., D. A. Krueger and G. International Tuberous Sclerosis Complex Consensus (2013). "Tuberous sclerosis complex diagnostic criteria update: recommendations of the 2012 linternational Tuberous Sclerosis Complex Consensus Conference." Pediatr Neurol 49(4): 243-254.

Northrup, H., D. A. Krueger and G. International Tuberous Sclerosis Complex Consensus (2013). "Tuberous sclerosis complex diagnostic criteria update: recommendations of the 2012 international tuberous sclerosis complex consensus conference." Pediatr Neurol 49(4): 243-254.

Northrup, H., J. W. Wheless, T. K. Bertin and R. A. Lewis (1993). "Variability of expression in tuberous sclerosis." J Med Genet 30(1): 4143. 
O'Callaghan, F. J., A. W. Shiell, J. P. Osborne and C. N. Martyn (1998). "Prevalence of tuberous sclerosis estimated by capture-recapture analysis." Lancet 351(9114): 1490.

Orlova, K. A. and P. B. Crino (2010). "The tuberous sclerosis complex." Ann N Y Acad Sci 1184: 87-105.

Osborne, J. P., A. Fryer and D. Webb (1991). "Epidemiology of tuberous sclerosis." Ann N Y Acad Sci 615: 125-127.

Oyazato, Y., K. lijima, M. Emi, T. Sekine, K. Kamei, J. Takanashi, H. Nakao, Y. Namai, K. Nozu and M. Matsuo (2011). "Molecular analysis of TSC2/PKD1 contiguous gene deletion syndrome." Kobe J Med Sci 57(1): E1-10.

Padma Priya, T. and A. B. Dalal (2012). "Tuberous sclerosis: diagnosis and prenatal diagnosis by MLPA." Indian J Pediatr 79(10): 13661369.

Panaretou, B., C. Prodromou, S. M. Roe, R. O'Brien, J. E. Ladbury, P. W. Piper and L. H. Pearl (1998). "ATP binding and hydrolysis are essential to the function of the Hsp90 molecular chaperone in vivo." EMBO J 17(16): 4829-4836.

Panaretou, B., G. Siligardi, P. Meyer, A. Maloney, J. K. Sullivan, S. Singh, S. H. Millson, P. A. Clarke, S. Naaby-Hansen, R. Stein, R. Cramer, M. Mollapour, P. Workman, P. W. Piper, L. H. Pearl and C. Prodromou (2002). "Activation of the ATPase activity of hsp90 by the stressregulated cochaperone aha1." Mol Cell 10(6): 1307-1318.

Peron, A., K. S. Au and H. Northrup (2018). "Genetics, genomics, and genotype-phenotype correlations of TSC: Insights for clinical practice." Am J Med Genet C Semin Med Genet 178(3): 281-290.

Piva, F., M. Giulietti, A. B. Burini and G. Principato (2012). "SpliceAid 2: a database of human splicing factors expression data and RNA target motifs." Hum Mutat 33(1): 81-85.

Plank, T. L., R. S. Yeung and E. P. Henske (1998). "Hamartin, the product of the tuberous sclerosis 1 (TSC1) gene, interacts with tuberin and appears to be localized to cytoplasmic vesicles." Cancer Research 58(21): 4766-4770.

Pollizzi, K., I. Malinowska-Kolodziej, C. Doughty, C. Betz, J. Ma, J. Goto and D. J. Kwiatkowski (2009). "A hypomorphic allele of Tsc2 highlights the role of TSC1/TSC2 in signaling to AKT and models mild human TSC2 alleles." Hum Mol Genet 18(13): 2378-2387.

Potter, C. J., H. Huang and T. Xu (2001). "Drosophila Tsc1 functions with Tsc2 to antagonize insulin signaling in regulating cell growth, cell proliferation, and organ size." Cell 105(3): 357-368.

Prabhakar, S., X. Zhang, J. Goto, S. Han, C. Lai, R. Bronson, M. Sena-Esteves, V. Ramesh, A. Stemmer-Rachamimov, D. J. Kwiatkowski and X. O. Breakefield (2015). "Survival benefit and phenotypic improvement by hamartin gene therapy in a tuberous sclerosis mouse brain model." Neurobiol Dis 82: 22-31.

Prodromou, C. and L. H. Pearl (2003). "Structure and functional relationships of Hsp90." Curr Cancer Drug Targets 3(5): $301-323$.

Qin, J., Z. Wang, M. Hoogeveen-Westerveld, G. Shen, W. Gong, M. Nellist and W. Xu (2016). "Structural Basis of the Interaction between Tuberous Sclerosis Complex 1 (TSC1) and Tre2-Bub2-Cdc16 Domain Family Member 7 (TBC1D7)." J Biol Chem 291(16): 8591-8601.

Qin, W., P. Kozlowski, B. E. Taillon, P. Bouffard, A. J. Holmes, P. Janne, S. Camposano, E. Thiele, D. Franz and D. J. Kwiatkowski (2010). "Ultra deep sequencing detects a low rate of mosaic mutations in tuberous sclerosis complex." Hum Genet 127(5): 573-582.

Rakowski, S. K., E. B. Winterkorn, E. Paul, D. J. Steele, E. F. Halpern and E. A. Thiele (2006). "Renal manifestations of tuberous sclerosis complex: Incidence, prognosis, and predictive factors." Kidney Int 70(10): 1777-1782. 
Rendtorff, N. D., B. Bjerregaard, M. Frodin, S. Kjaergaard, H. Hove, F. Skovby, K. Brondum-Nielsen, M. Schwartz and G. Danish Tuberous Sclerosis (2005). "Analysis of 65 tuberous sclerosis complex (TSC) patients by TSC2 DGGE, TSC1/TSC2 MLPA, and TSC1 long-range PCR sequencing, and report of 28 novel mutations." Hum Mutat 26(4): 374-383.

Richards, S., N. Aziz, S. Bale, D. Bick, S. Das, J. Gastier-Foster, W. W. Grody, M. Hegde, E. Lyon, E. Spector, K. Voelkerding, H. L. Rehm and A. L. Q. A. Committee (2015). "Standards and guidelines for the interpretation of sequence variants: a joint consensus recommendation of the American College of Medical Genetics and Genomics and the Association for Molecular Pathology." Genet Med 17(5): 405-424.

Riemenschneider, M. J., R. A. Betensky, S. M. Pasedag and D. N. Louis (2006). "AKT activation in human glioblastomas enhances proliferation via TSC2 and S6 kinase signaling." Cancer Res 66(11): 5618-5623.

Rio, D., S. Clark and R. Tjian (1985). "A mammalian host-vector system that regulates expression and amplification of transfected genes by temperature induction." Science 227(4682): 23-28.

Roach, E. S., M. R. Gomez and H. Northrup (1998). "Tuberous sclerosis complex consensus conference: revised clinical diagnostic criteria." J Child Neurol 13(12): 624-628.

Roach, E. S. and S. P. Sparagana (2004). "Diagnosis of tuberous sclerosis complex." J Child Neurol 19(9): 643-649.

Rosset, C., F. Vairo, I. C. Bandeira, R. L. Correia, F. V. de Goes, R. T. B. da Silva, L. S. M. Bueno, M. C. S. de Miranda Gomes, H. C. R. Galvao, J. Neri, M. I. Achatz, C. B. O. Netto and P. Ashton-Prolla (2017). "Molecular analysis of TSC1 and TSC2 genes and phenotypic correlations in Brazilian families with tuberous sclerosis." PLoS One 12(10): e0185713.

Rozen, S. and H. J. Skaletsky (2000). "Primer3 on the WWW for general users and for biologist programmers. In: Krawetz S, Misener S (eds) Bioinformatics Methods and Protocols: Methods in Molecular Biology." Humana Press: 2.

Sager, R. A., M. R. Woodford and M. Mollapour (2018). "The mTOR Independent Function of Tsc1 and FNIPs." Trends Biochem Sci.

Sager, R. A., M. R. Woodford, O. Shapiro, M. Mollapour and G. Bratslavsky (2018). "Sporadic renal angiomyolipoma in a patient with BirtHogg-Dube: chaperones in pathogenesis." Oncotarget 9(31): 22220-22229.

Sampson, J. R., S. J. Scahill, J. B. Stephenson, L. Mann and J. M. Connor (1989). "Genetic aspects of tuberous sclerosis in the west of Scotland." J Med Genet 26(1): 28-31.

Sancak, O., M. Nellist, M. Goedbloed, P. Elfferich, C. Wouters, A. Maat-Kievit, B. Zonnenberg, S. Verhoef, D. Halley and A. van den Ouweland (2005). "Mutational analysis of the TSC1 and TSC2 genes in a diagnostic setting: genotype--phenotype correlations and comparison of diagnostic DNA techniques in Tuberous Sclerosis Complex." Eur J Hum Genet 13(6): 731-741.

Santiago Lima, A. J., M. Hoogeveen-Westerveld, A. Nakashima, A. Maat-Kievit, A. van den Ouweland, D. Halley, U. Kikkawa and M. Nellist (2014). "Identification of regions critical for the integrity of the TSC1-TSC2-TBC1D7 complex." PLoS One 9(4): e93940.

Sarbassov, D. D., D. A. Guertin, S. M. Ali and D. M. Sabatini (2005). "Phosphorylation and regulation of Akt/PKB by the rictor-mTOR complex." Science 307(5712): 1098-1101.

Sasongko, T. H., M. Wataya-Kaneda, K. Koterazawa, Gunadi, S. Yusoff, I. S. Harahap, M. J. Lee, M. Matsuo and H. Nishio (2008). "Novel mutations in 21 patients with tuberous sclerosis complex and variation of tandem splice-acceptor sites in TSC1 exon 14." Kobe J Med Sci 54(1): E73-81.

Scheithauer, B. J. and T. J. Reagan (1999). "In: Gomez, M. R. e Whittemore, V. H. 3a Ed. Tuberous Sclerois Complex." Neurophatology: 44.

Schubert, U., L. C. Anton, J. Gibbs, C. C. Norbury, J. W. Yewdell and J. R. Bennink (2000). "Rapid degradation of a large fraction of newly synthesized proteins by proteasomes." Nature 404(6779): 770-774. 
Schwarz, J. M., C. Rodelsperger, M. Schuelke and D. Seelow (2010). "MutationTaster evaluates disease-causing potential of sequence alterations." Nat Methods 7(8): 575-576.

Schwarze, K., J. Buchanan, J. C. Taylor and S. Wordsworth (2018). "Are whole-exome and whole-genome sequencing approaches costeffective? A systematic review of the literature." Genet Med 20(10): 1122-1130.

Shepherd, C. W., O. W. Houser and M. R. Gomez (1995). "MR findings in tuberous sclerosis complex and correlation with seizure development and mental impairment." AJNR Am J Neuroradiol 16(1): 149-155.

Shepherd, C. W., B. W. Scheithauer, M. R. Gomez, H. J. Altermatt and J. A. Katzmann (1991). "Subependymal giant cell astrocytoma: a clinical, pathological, and flow cytometric study." Neurosurgery 28(6): 864-868.

Siroky, B. J., H. Yin, J. T. Babcock, L. Lu, A. R. Hellmann, B. P. Dixon, L. A. Quilliam and J. J. Bissler (2012). "Human TSC-associated renal angiomyolipoma cells are hypersensitive to ER stress." Am J Physiol Renal Physiol 303(6): F831-844.

Smith, E. M., S. G. Finn, A. R. Tee, G. J. Browne and C. G. Proud (2005). "The tuberous sclerosis protein TSC2 is not required for the regulation of the mammalian target of rapamycin by amino acids and certain cellular stresses." J Biol Chem 280(19): 18717-18727.

Smolarek, T. A., L. L. Wessner, F. X. McCormack, J. C. Mylet, A. G. Menon and E. P. Henske (1998). "Evidence that lymphangiomyomatosis is caused by TSC2 mutations: chromosome $16 \mathrm{p} 13$ loss of heterozygosity in angiomyolipomas and lymph nodes from women with lymphangiomyomatosis." Am J Hum Genet 62(4): 810-815.

Stefansson, K. (1991). "Tuberous sclerosis." Mayo Clin Proc 66(8): 868-872.

Tee, A. R., D. C. Fingar, B. D. Manning, D. J. Kwiatkowski, L. C. Cantley and J. Blenis (2002). "Tuberous sclerosis complex-1 and -2 gene products function together to inhibit mammalian target of rapamycin (mTOR)-mediated downstream signaling." Proc Natl Acad Sci U S A 99(21): 13571-13576.

Tee, A. R., B. D. Manning, P. P. Roux, L. C. Cantley and J. Blenis (2003). "Tuberous Sclerosis Complex Gene Products, Tuberin and Hamartin, Control mTOR Signaling by Acting as a GTPase-Activating Protein Complex toward Rheb." Current Biology 13(15): 1259-1268.

Thiele, E. A. (2004). "Managing epilepsy in tuberous sclerosis complex." J Child Neurol 19(9): 680-686.

Trombley, I. K. and S. S. Mirra (1981). "Ultrastructure of tuberous sclerosis: cortical tuber and subependymal tumor." Ann Neurol 9(2): 174-181.

Tyburczy, M. E., K. A. Dies, J. Glass, S. Camposano, Y. Chekaluk, A. R. Thorner, L. Lin, D. Krueger, D. N. Franz, E. A. Thiele, M. Sahin and D. J. Kwiatkowski (2015). "Mosaic and Intronic Mutations in TSC1/TSC2 Explain the Majority of TSC Patients with No Mutation Identified by Conventional Testing." PLoS Genet 11(11): e1005637.

Tyburczy, M. E., J. A. Wang, S. Li, R. Thangapazham, Y. Chekaluk, J. Moss, D. J. Kwiatkowski and T. N. Darling (2013). "Sun exposure causes somatic second-hit mutations and angiofibroma development in tuberous sclerosis complex." Human Molecular Genetics 23(8): 20232029.

Tyburczy, M. E., J. A. Wang, S. Li, R. Thangapazham, Y. Chekaluk, J. Moss, D. J. Kwiatkowski and T. N. Darling (2014). "Sun exposure causes somatic second-hit mutations and angiofibroma development in tuberous sclerosis complex." Hum Mol Genet 23(8): $2023-2029$.

van den Ouweland, A. M., P. Elfferich, B. A. Zonnenberg, W. F. Arts, T. Kleefstra, M. D. Nellist, J. M. Millan, C. Withagen-Hermans, A. J. Maat-Kievit and D. J. Halley (2011). "Characterisation of TSC1 promoter deletions in tuberous sclerosis complex patients." Eur J Hum Genet 19(2): 157-163. 
van Dijk, M. C., P. D. Rombout, S. H. Boots-Sprenger, H. Straatman, M. R. Bernsen, D. J. Ruiter and J. W. Jeuken (2005). "Multiplex ligationdependent probe amplification for the detection of chromosomal gains and losses in formalin-fixed tissue." Diagn Mol Pathol 14(1): 916.

van Eeghen, A. M., L. O. Teran, J. Johnson, M. B. Pulsifer, E. A. Thiele and P. Caruso (2013). "The neuroanatomical phenotype of tuberous sclerosis complex: focus on radial migration lines." Neuroradiology 55(8): 1007-1014.

van Slegtenhorst, M., R. de Hoogt, C. Hermans, M. Nellist, B. Janssen, S. Verhoef, D. Lindhout, A. van den Ouweland, D. Halley, J. Young, M. Burley, S. Jeremiah, K. Woodward, J. Nahmias, M. Fox, R. Ekong, J. Osborne, J. Wolfe, S. Povey, R. G. Snell, J. P. Cheadle, A. C. Jones, M. Tachataki, D. Ravine, J. R. Sampson, M. P. Reeve, P. Richardson, F. Wilmer, C. Munro, T. L. Hawkins, T. Sepp, J. B. Ali, S. Ward, A. J. Green, J. R. Yates, J. Kwiatkowska, E. P. Henske, M. P. Short, J. H. Haines, S. Jozwiak and D. J. Kwiatkowski (1997). "Identification of the tuberous sclerosis gene TSC1 on chromosome 9q34." Science 277(5327): 805-808.

van Slegtenhorst, M., M. Nellist, B. Nagelkerken, J. Cheadle, R. Snell, A. van den Ouweland, A. Reuser, J. Sampson, D. Halley and P. van der Sluijs (1998). "Interaction between hamartin and tuberin, the TSC1 and TSC2 gene products." Human molecular genetics 7(6): 10531057 .

van Slegtenhorst, M., M. Nellist, B. Nagelkerken, J. Cheadle, R. Snell, A. van den Ouweland, A. Reuser, J. Sampson, D. Halley and P. van der Sluijs (1998). "Interaction between hamartin and tuberin, the TSC1 and TSC2 gene products." Hum Mol Genet 7(6): 7.

van Slegtenhorst, M. A., A. Verhoef, A. Tempelaars, L. Bakker, Q. Wang, M. Wessels, R. Bakker, M. Nellist, D. Lindhout, D. Halley and A. van den Ouweland (1999). "Mutational spectrum of the TSC1 gene in a cohort of 225 tuberous sclerosis complex patients: no evidence for genotype-phenotype correlation." J Med Genet 36(4): 5.

von Ranke, F. M., I. M. Faria, G. Zanetti, B. Hochhegger, A. S. Souza, Jr. and E. Marchiori (2017). "Imaging of tuberous sclerosis complex: a pictorial review." Radiol Bras 50(1): 48-54.

Wang, K., M. Li and H. Hakonarson (2010). "ANNOVAR: functional annotation of genetic variants from high-throughput sequencing data." Nucleic Acids Research 38(16): e164-e164.

Webb, D. W. and J. P. Osborne (1991). "Non-penetrance in tuberous sclerosis." J Med Genet 28(6): 417-419.

Weeks, D. A., R. L. Malott, M. Arnesen, C. Zuppan, D. Aitken and G. Mierau (1991). "Hepatic angiomyolipoma with striated granules and positivity with melanoma--specific antibody (HMB-45): a report of two cases." Ultrastruct Pathol 15(4-5): 563-571.

Weren, R. D., M. J. Ligtenberg, C. M. Kets, R. M. de Voer, E. T. Verwiel, L. Spruijt, W. A. van Zelst-Stams, M. C. Jongmans, C. Gilissen, J. Y. Hehir-Kwa, A. Hoischen, J. Shendure, E. A. Boyle, E. J. Kamping, I. D. Nagtegaal, B. B. Tops, F. M. Nagengast, A. Geurts van Kessel, J. H. van Krieken, R. P. Kuiper and N. Hoogerbrugge (2015). "A germline homozygous mutation in the base-excision repair gene NTHL1 causes adenomatous polyposis and colorectal cancer." Nat Genet 47(6): 668-671.

Wiederholt, W. C., M. R. Gomez and L. T. Kurland (1985). "Incidence and prevalence of tuberous sclerosis in Rochester, Minnesota, 1950 through 1982." Neurology 35(4): 3

Wildeman, M., E. van Ophuizen, J. T. den Dunnen and P. E. Taschner (2008). "Improving sequence variant descriptions in mutation databases and literature using the Mutalyzer sequence variation nomenclature checker." Hum Mutat 29(1): 6-13.

Wilson, P. J., V. Ramesh, A. Kristiansen, C. Bove, S. Jozwiak, D. J. Kwiatkowski, M. P. Short and J. L. Haines (1996). "Novel Mutations Detected in the TSC2 Hene From Both Sporadic and Familial TSC Patients." Human Molecular Genetics 5(2): 249-256.

Woodford, M. R., D. M. Dunn, A. R. Blanden, D. Capriotti, D. Loiselle, C. Prodromou, B. Panaretou, P. F. Hughes, A. Smith, W. Ackerman, T. A. Haystead, S. N. Loh, D. Bourboulia, L. S. Schmidt, W. Marston Linehan, G. Bratslavsky and M. Mollapour (2016). "The FNIP cochaperones decelerate the Hsp90 chaperone cycle and enhance drug binding." Nat Commun 7: 12037. 
Woodford, M. R., R. A. Sager, E. Marris, D. M. Dunn, A. R. Blanden, R. L. Murphy, N. Rensing, O. Shapiro, B. Panaretou, C. Prodromou, S. N. Loh, D. H. Gutmann, D. Bourboulia, G. Bratslavsky, M. Wong and M. Mollapour (2017). "Tumor suppressor Tsc1 is a new Hsp90 cochaperone that facilitates folding of kinase and non-kinase clients." EMBO J 36(24): 3650-3665.

Yang, L., X. L. Feng, S. Shen, L. Shan, H. F. Zhang, X. Y. Liu and N. LV (2012). "Clinicopathological analysis of 156 patients with angiomyolipoma originating from different organs." Oncol Lett 3(3): 586-590.

Zerbino, D. R., P. Achuthan, W. Akanni, M. R. Amode, D. Barrell, J. Bhai, K. Billis, C. Cummins, A. Gall, C. G. Giron, L. Gil, L. Gordon, L. Haggerty, E. Haskell, T. Hourlier, O. G. Izuogu, S. H. Janacek, T. Juettemann, J. K. To, M. R. Laird, I. Lavidas, Z. Liu, J. E. Loveland, T. Maurel, W. McLaren, B. Moore, J. Mudge, D. N. Murphy, V. Newman, M. Nuhn, D. Ogeh, C. K. Ong, A. Parker, M. Patricio, H. S. Riat, H. Schuilenburg, D. Sheppard, H. Sparrow, K. Taylor, A. Thormann, A. Vullo, B. Walts, A. Zadissa, A. Frankish, S. E. Hunt, M. Kostadima, N. Langridge, F. J. Martin, M. Muffato, E. Perry, M. Ruffier, D. M. Staines, S. J. Trevanion, B. L. Aken, F. Cunningham, A. Yates and P. Flicek (2018). "Ensembl 2018." Nucleic Acids Res 46(D1): D754-D761.

Zhang, H., G. Cicchetti, H. Onda, H. B. Koon, K. Asrican, N. Bajraszewski, F. Vazquez, C. L. Carpenter and D. J. Kwiatkowski (2003). "Loss of Tsc1/Tsc2 activates mTOR and disrupts PI3K-Akt signaling through downregulation of PDGFR." J Clin Invest 112(8): 1223-1233.

Zhang, Y., X. Gao, L. J. Saucedo, B. Ru, B. A. Edgar and D. Pan (2003). "Rheb is a direct target of the tuberous sclerosis tumour suppressor proteins." Nat Cell Biol 5(6): 578-581. 


\section{Supplementary Tables}

Supplementary Table III-1: RNA sample information. RQN: RNA quality number. QC: Quality control. All samples passed on RQN and QC.

\begin{tabular}{|c|c|c|c|}
\hline Customer ID & Material Type & RQN & Entry QC (ng/ul) \\
\hline 293T $(\mathrm{DMEM}+/+)$ & total RNA & 10 & 1551.86 \\
\hline $1 \mathrm{C} 2(\mathrm{DMEM}+/+)$ & total RNA & 10 & 1262.16 \\
\hline 3H9 (DMEM +/+) & total RNA & 10 & 1582.19 \\
\hline 3H9-1B1 (DMEM +/+) & total RNA & 10 & 1197.36 \\
\hline 293T (DMEM -/-) & total RNA & 10 & 1138.53 \\
\hline $1 \mathrm{C} 2$ (DMEM -/-) & total RNA & 10 & 885.78 \\
\hline 3H9 (DMEM -/-) & total RNA & 10 & 501.64 \\
\hline 3H9-1B1 (DMEM -/-) & total RNA & 10 & 754.1 \\
\hline $293 \mathrm{~T}(\mathrm{DMEM}+/+)$ & total RNA & 10 & 981.95 \\
\hline $3 \mathrm{C} 2(\mathrm{DMEM}+/+)$ & total RNA & 10 & 911.37 \\
\hline 3A1 (DMEM +/+) & total RNA & 10 & 1016.13 \\
\hline 3H9-1C4 (DMEM +/+) & total RNA & 10 & 773.92 \\
\hline 293 T (DMEM -/-) & total RNA & 10 & 858.66 \\
\hline 3C2 (DMEM -/-) & total RNA & 10 & 621.08 \\
\hline 3A1 (DMEM -/-) & total RNA & 10 & 816.27 \\
\hline 3H9-1C4 (DMEM -/-) & total RNA & 10 & 960.41 \\
\hline 293T $(\mathrm{DMEM}+/+)$ & total RNA & 10 & 867.13 \\
\hline 3C2 (DMEM +/+) & total RNA & 10 & 768.35 \\
\hline $1 \mathrm{C} 2(\mathrm{DMEM}+/+)$ & total RNA & 10 & 1023.06 \\
\hline 3A1 (DMEM +/+) & total RNA & 10 & 547.62 \\
\hline 3H9 (DMEM +/+) & total RNA & 10 & 1357.19 \\
\hline 3H9-1C4 (DMEM +/+) & total RNA & 10 & 466.22 \\
\hline 3H9-1B1 (DMEM +/+) & total RNA & 10 & 774.4 \\
\hline 293T (DMEM -/-) & total RNA & 10 & 911.17 \\
\hline 3C2 (DMEM -/-) & total RNA & 10 & 887.26 \\
\hline $1 \mathrm{C} 2$ (DMEM -/-) & total RNA & 10 & 871.46 \\
\hline 3A1 (DMEM -/-) & total RNA & 10 & 1108.38 \\
\hline 3H9 (DMEM -/-) & total RNA & 10 & 1198.26 \\
\hline 3H9-1C4 (DMEM -/-) & total RNA & 10 & 1134.05 \\
\hline 3H9-1B1 (DMEM -/-) & total RNA & 10 & 825.42 \\
\hline
\end{tabular}


Supplementary Table III-2: Library construction and Molarity.

\begin{tabular}{|c|c|}
\hline Customer ID & Molarity (nM) \\
\hline 293T $(\mathrm{DMEM}+/+)$ & 5.33 \\
\hline 1C2 (DMEM +/+) & 3.75 \\
\hline 3H9 (DMEM +/+) & 4.40 \\
\hline 3H9-1B1 (DMEM +/+) & 5.00 \\
\hline 293T (DMEM -/-) & 7.30 \\
\hline 1C2 (DMEM -/-) & 7.57 \\
\hline 3H9 (DMEM -/-) & 8.10 \\
\hline 3H9-1B1 (DMEM -/-) & 4.28 \\
\hline 293T $(\mathrm{DMEM}+/+)$ & 6.82 \\
\hline $3 \mathrm{C} 2(\mathrm{DMEM}+/+)$ & 7.05 \\
\hline 3A1 (DMEM +/+) & 10.40 \\
\hline 3H9-1C4 (DMEM +/+) & 4.44 \\
\hline 293T (DMEM -/-) & 6.89 \\
\hline 3C2 (DMEM -/-) & 6.56 \\
\hline 3A1 (DMEM -/-) & 7.13 \\
\hline 3H9-1C4 (DMEM -/-) & 9.10 \\
\hline 293T $(\mathrm{DMEM}+/+)$ & 6.62 \\
\hline $3 \mathrm{C} 2(\mathrm{DMEM}+/+)$ & 5.05 \\
\hline $1 \mathrm{C} 2(\mathrm{DMEM}+/+)$ & 7.48 \\
\hline 3A1 (DMEM +/+) & 6.44 \\
\hline 3H9 (DMEM +/+) & 7.08 \\
\hline 3H9-1C4 (DMEM +/+) & 9.79 \\
\hline 3H9-1B1 (DMEM +/+) & 4.23 \\
\hline 293T (DMEM -/-) & 5.32 \\
\hline 3C2 (DMEM -/-) & 4.71 \\
\hline 1C2 (DMEM -/-) & 5.01 \\
\hline 3A1 (DMEM -/-) & 6.72 \\
\hline 3H9 (DMEM -/-) & 3.68 \\
\hline 3H9-1C4 (DMEM -/-) & 3.56 \\
\hline 3H9-1B1 (DMEM -/-) & 3.13 \\
\hline
\end{tabular}


Supplementary Table III-3: Run yields, clusters and percentage of Q30 per sample.

\begin{tabular}{|c|c|c|c|}
\hline Sample ID & Yield (Mb) & Clusters & $\%>=\mathrm{Q} 30$ \\
\hline $293 \mathrm{~T}(\mathrm{DMEM}+/+)$ & 4,359 & $14,435,335$ & 90.68 \\
\hline $1 \mathrm{C} 2(\mathrm{DMEM}+/+)$ & 3,543 & $11,731,379$ & 90.14 \\
\hline 3H9 (DMEM +/+) & 4,518 & $14,957,866$ & 91.35 \\
\hline 3H9-1B1 (DMEM +/+) & 4,192 & $13,881,889$ & 90.68 \\
\hline 293T (DMEM -/-) & 4,357 & $14,428,154$ & 90.83 \\
\hline $1 \mathrm{C} 2$ (DMEM -/-) & 4,204 & $13,918,424$ & 90.96 \\
\hline 3H9 (DMEM -/-) & 4,281 & $14,175,446$ & 90.64 \\
\hline 3H9-1B1 (DMEM -/-) & 3,578 & $11,847,647$ & 90.72 \\
\hline $293 \mathrm{~T}(\mathrm{DMEM}+/+)$ & 3,835 & $12,698,726$ & 90.25 \\
\hline $3 \mathrm{C} 2(\mathrm{DMEM}+/+)$ & 3,611 & $11,954,942$ & 90.54 \\
\hline 3A1 $($ DMEM +/+) & 3,984 & $13,191,862$ & 90.69 \\
\hline 3H9-1C4 (DMEM +/+) & 3,970 & $13,146,674$ & 89.93 \\
\hline 293 T (DMEM -/-) & 4,052 & $13,416,176$ & 90.31 \\
\hline 3C2 (DMEM -/-) & 3,840 & $12,715,334$ & 90.37 \\
\hline 3A1 (DMEM -/-) & 3,554 & $11,770,421$ & 90.06 \\
\hline 3H9-1C4 (DMEM -/-) & 5,331 & $17,651,436$ & 90.11 \\
\hline $293 \mathrm{~T}(\mathrm{DMEM}+/+)$ & 6,269 & $20,757,887$ & 91.08 \\
\hline 3C2 (DMEM +/+) & 4,114 & $13,620,545$ & 90.06 \\
\hline $1 \mathrm{C} 2(\mathrm{DMEM}+/+)$ & 6,498 & $21,514,943$ & 90.54 \\
\hline 3A1 (DMEM +/+) & 4,255 & $14,088,555$ & 90.05 \\
\hline 3H9 (DMEM +/+) & 3,561 & $11,791,372$ & 89.18 \\
\hline 3H9-1C4 (DMEM +/+) & 3,894 & $12,895,789$ & 90.23 \\
\hline 3H9-1B1 (DMEM +/+) & 3,529 & $11,685,430$ & 89.36 \\
\hline 293T (DMEM -/-) & 3,711 & $12,287,753$ & 90.18 \\
\hline 3C2 (DMEM -/-) & 7,429 & $24,598,652$ & 89.78 \\
\hline 1C2 (DMEM -/-) & 3,477 & $11,513,170$ & 89.65 \\
\hline 3A1 (DMEM -/-) & 5,368 & $17,775,044$ & 89.78 \\
\hline 3H9 (DMEM -/-) & 4,043 & $13,384,615$ & 88.99 \\
\hline 3H9-1C4 (DMEM -/-) & 3,959 & $13,110,984$ & 89.72 \\
\hline 3H9-1B1 (DMEM -/-) & 3,940 & $13,045,184$ & 89.55 \\
\hline
\end{tabular}




\section{Supplementary Figures}

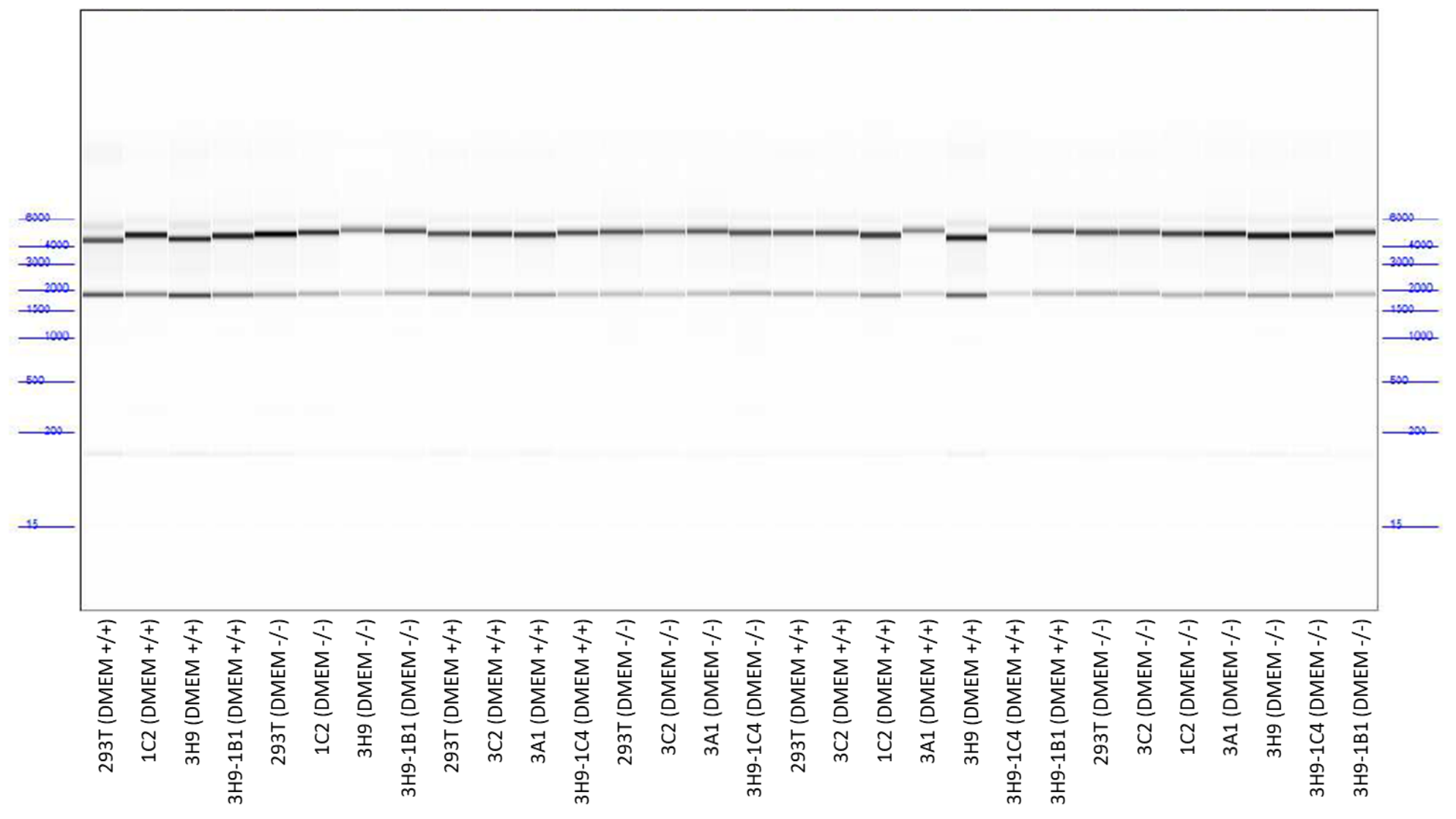

Supplementary Figure III-1: Gel image of total RNA fragment analysis. Separation at $8.0 \mathrm{kV}$, for 40.0 min. 


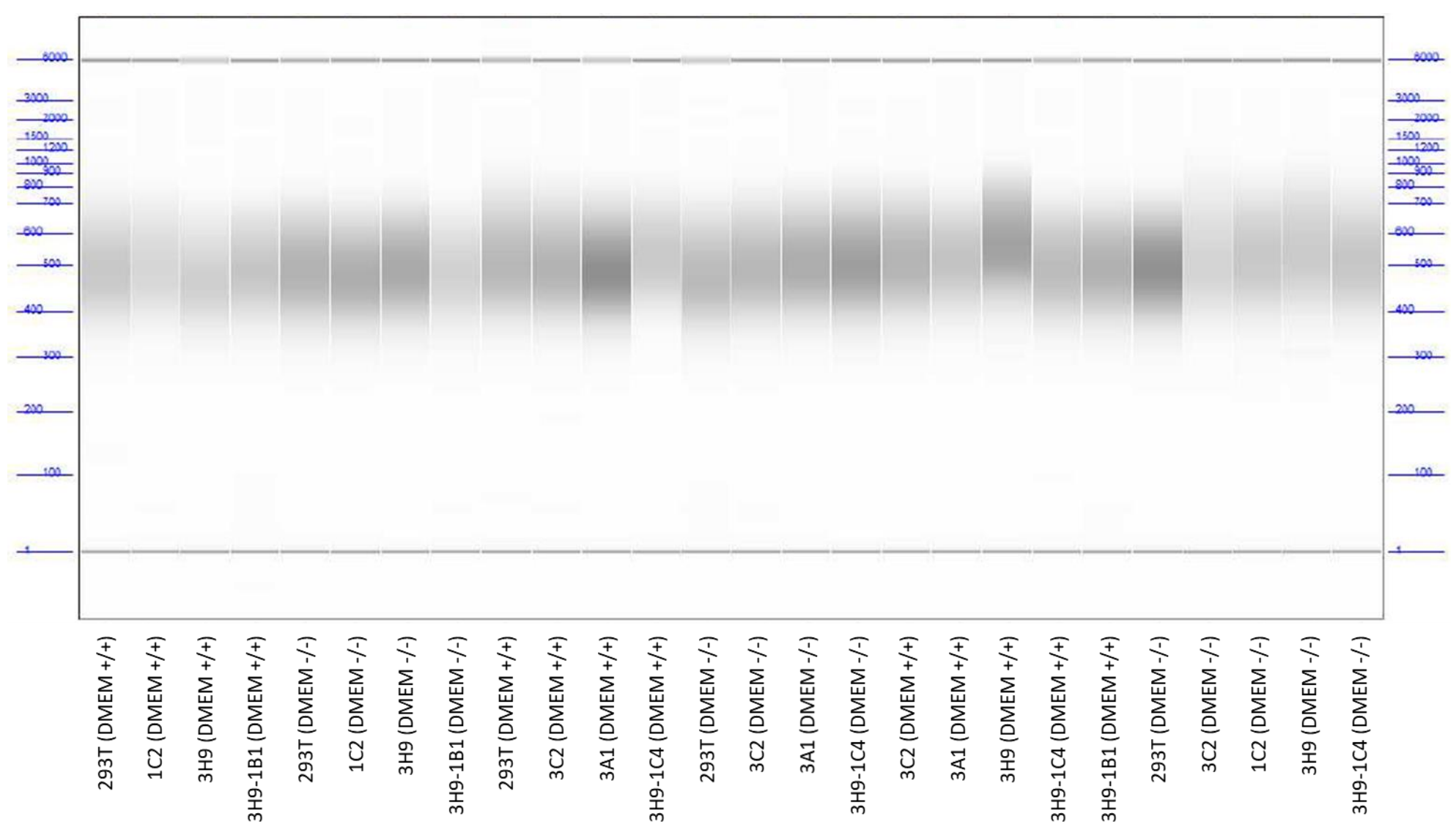

Supplementary Figure III-2: Gel image of NGS Fragment 1-6000 bp analysis. Separation at 6.0 kV, for 50.0 min. 
Portfolio 


\section{Complementary Studies}

1. 2017: Short course in NGS in DNA Diagnostics Course. (Course load: 24h). Erasmus Medical Center, ERASMUS MC, Rotterdam, Holand

2. 2016: Short course in New Resources at the UCSC Genome Browser for the Display and Interpretation. (Course load: 2h). American Society Of Human Genetics, Bethesda, United States of America

3. 2015: Short course in Genome Editing. (Course load: 6h). Latin American School of Human and Medical Genetics, ELAG, Brazil

4. 2015: Short course in Next Generation Sequencing. (Course load: 6h). Latin American School of Human and Medical Genetics, ELAG, Brazil

5. 2014: Short course in iSeqTools to demistify the cloud and genomics. (Course load: 4h). American Society of Human Genetics, ASHG, United States of America

6. 2014: Short course in Principio da Técnica de interferências por RNA. (Course load: 4h). Sociedade Brasileira de Genética, SBG, Ribeirao Preto, Brazil

7. 2014: Short course in Ensembl Highlights: Intermediate/Advanced Workshop. (Course load: 4h). American Society of Human Genetics, ASHG, United States of America

\section{Chapters of published books}

1. Dufner-Almeida, Luiz Gustavo; do Carmo, Ramon Torreglosa; Masotti, Cibele; Haddad, L A, Understanding human DNA variants affecting pre-mRNA splicing in the NGS era In: Advances in Genetics.103 ed.: Elsevier, 2019, p. 39-90. ISBN: 9780128171592, Home page: https://linkinghub.elsevier.com/retrieve/pii/S0065266018300348 (Appendix 3)

\section{Complete articles published in journals}

1. Almeida, Luiz Gustavo Dufner; Cruz, Dayane Bernardino da; Mingroni Netto, Regina Célia; Batissoco, Ana Carla; Ferraz, Jeane Oiticica Ramalho; Silva, Rodrigo Salazar, Stem-cell therapy for hearing loss: are we there yet?. Brazilian Journal of Otorhinolaryngology, v.85, p.520 - 529, 2019. [doi:10.1016/j.bjorl.2019.04.006] (Appendix 4)

\section{Papers published in event annals}

1. Dufner-Almeida, L.G.; ALmeida, J. P. G.; Masulk, L.; Richards, M.; Miranda, N.; Silva, I. M. W.; Chiavegatto, S.; Passos-Bueno, M. R.; Antoniuki, S. A.; Rosemberg, S.; Haddad, L A, Segmental genomic deletions and splicing mutations causing tuberous sclerosis complex (TSC) In: American Society of Human Genetics 66th Annual Meeting, 2016, Vancouver, BC, Canada. American Society of Human Genetics 66th Annual Meeting. , 2016. p.1 1996

2. Dufner-Almeida, L.G.; Almeida, J. P. G.; Rosemberg, S.; Haddad, Luciana A H, A relatively high rate of TSC1 mutations causing tuberous sclerosis complex (TSC) in a cohort of 21 patients In: XI Course of the Latin American School of Human and Medical Genetics, 2015, Caxias do Sul, RS., XI Course of the Latin American School of Human and Medical Genetics2015. 
3. Dufner-Almeida, L.G.; Almeida, J. P. G.; Masulk, L.; Richardz, M.; Miranda, N.; Antoniuk, S. A.; Rosemberg, S.; HADDAD, L. A. Tuberous sclerosis complex-causing mutations analyzed in a cohort of 41 Brazilian patients In: ASHG 65th Annual Meeting, 2015, Baltimore, MD, USA. ASHG 65th Annual Meeting, 2015.

4. Dufner-Almeida, L.G.; Almeida, J. P. G.; Masulk, L.; Richardz, M.; Miranda, N.; Antoniuk, S. A.; Rosemberg, S.; HADDAD, L. A., Analyses of TSC genes among Brazilian tuberous sclerosis complex (TSC) patients In: ASHG 64th Annual Meeting, 2014, San Diego, Ca, USA. ASHG 64th Annual Meeting. 2014.

\section{Presentation of work and lecture}

1. Nanhoe, S.; Dufner-Almeida, L.G.; Elfferich, P.; Hoogeveen-Westerveld, M.; Swenker, R.; Hossinzadeh, M.; KomGortat, R.; Brouwer, R.; Ijcken, W. V.; Ekong, R.; Povey, S.; Nellist, M., Improving molecular diagnostics for tuberous sclerosis complex, 2017, International TSC symposium

2. Dufner-Almeida, L.G.; Almeida, J. P. G.; Masulk, L.; Richardz, M.; Miranda, N.; Silvia, I. M. W.; Chiavegatto, S.; Passos-Bueno, M. R.; Antoniuk, S. A.; Rosemberg, S.; Haddad, L. A., Segmental genomic deletions and splicing mutations causing tuberous sclerosis complex (TSC), 2016, Vancouver, BC, Canada; American Society of Human Genetics 66th Annual Meeting

3. Carvalho, D. S.; Antoniuk, S. A.; Cardozo, L. F. M.; Bruck, I.; Haddad, L. A.; Dufner-Almeida, L.G., Tuberous Sclerosis Complex: Clinical Evaluation in 47 patients, 2016, Lisboa.

4. Dufner-Almeida, L.G.; Almeida, J. P. G.; Rosemberg, S.; Haddad, L A, A relatively high rate of TSC1 mutation causing tuberous sclerosis complex (TSC) in a cohort of 21 patients, 2015, Brazil; Evento: XI Course of the Latin American School of Human and Medical Genetics

5. Dufner-Almeida, L.G.; Almeida, J. P. G.; Masulk, L.; Richardz, M.; Miranda, N.; Antoniuk, S. A.; Rosemberg, S.; Haddad, L A, Tuberous sclerosis complex-causing mutations analyzed in a cohort of 41 Brazilian patients, 2015, United States, ASHG 65th Annual Meeting

6. Dufner-Almeida, L.G.; Almeida, J. P. G.; Masulk, L.; Richardz, M.; Miranda, N.; Antoniuk, S. A.; Rosemberg, S.; Haddad, L. A., Analyses of TSC genes among Brazilian tuberous sclerosis complex (TSC) patients, 2014., United States, ASHG 64th Annual Meeting.

\section{Participation in events}

1. Lecture at ABET 2015, Mão na massa: entendendo porque a Esclerose Tuberosa se manifesta.

2. Poster / Panel Presentation in ASHG 65th Annual Meeting, 2015, Tuberous sclerosis complex-causing mutations analyzed in a cohort of 41 Brazilian patients.

3. Poster / Panel Presentation in XI Course of the Latin American School of Human and Medical Genetics, 2015, $A$ relatively high rate of TSC1 mutations causing tuberous sclerosis complex (TSC) in a cohort of 21 patients.

4. Poster / Panel Presentation in $60^{\circ}$ Congresso Brasileiro de Genética, 2014, TSC1 gene DNA variant analysis in tuberous sclerosis complex (TSC) patients.

5. Poster / Panel Presentation in ASHG 64th Annual Meeting, 2014, Analyses of TSC genes among Brazilian tuberous sclerosis complex (TSC) patients. 
Appendix 


\section{Appendix 1}
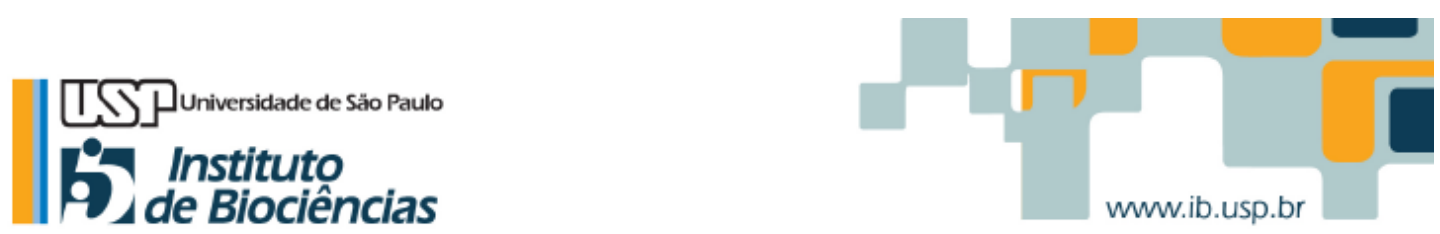

Nümero de Identificação: LGF

Projeto de pesquisa: Análise dos genes causadores do complexo da esclerose tuberosa

TERMO DE CONSENTIMENTO LIVRE E ESCLARECIDO

(Maiores de 18 anos)

COLETA DE SANGUE

Você está sendo convidado(a) a participar do projeło de pesquisa acima citado. Este documento contém todas as informaçôes necessárias sobre a pesquisa que estamos fazendo. Sua colaboração neste estudo será muito importante para entendermos um pouco mais sobre o complexo da esclerose tuberosa.

Eu, residente e domiciliado(a) na

inscrito(a) no CPF RG............................... e nascido(a) em ............... declaro que fui esclarecido(a) pelo portador(a) da cédula de identidade, pesquisador, entendi o que me foi explicado e tive oportunidade para lhe fazer perguntas sobre o projeto "Análise dos genes causadores do complexo da esclerose tuberosa".

Consinto de livre e espontânea vontade em participar do estudo "Análise dos genes causadores do complexo da esclerose tuberosa" e estou ciente que:

(a) O objetivo do estudo é identificar mutações associadas ao complexo da esclerose tuberosa e entender como a esclerose tuberosa se manifesta.

(b) Será feita coleta de sangue (cerca de 10 mililitros), o que poderá causar pequeno incômodo ou dor no momento da coleta (introdução da agulha na veia).

(c) O sangue se destinará à extração de DNA, que deverá ser armazenado em laboratório pelo tempo necessário e utilizado em pesquisa de mutaçōes associadas ao complexo da esclerose tuberosa.

(d) Uma alíquota codificada (sem identificação) do DNA em quantidade suficiente para esta análise poderá ser enviada a um laboratório especializado no exterior.

(e) A análise do sequenciamento do DNA será realizada somente para mutaçōes causadoras e modificadoras do complexo da esclerose tuberosa.

(f) Resultados dos meus exames clínico e laboratoriais poderão ser utilizados para fins de publicaçōes cientificas, aulas ou seminários científicos. No entanto, a publicação de imagens fotográficas só será possível com meu consentimento abaixo.

(g) Mutaçōes em qualquer segmento dos genes TSC1 ou TSC2 podem causar a esclerose tuberosa, o que faz a análise laboriosa e demorada. Por este motivo e por se tratar de um novo projeto de pesquisa, os resultados poderão ser obtidos nos próximos anos. 


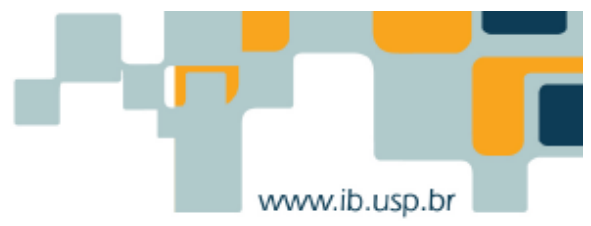

Número de Identificaçã $\alpha$ LGF

(h) Nas condiçôes atuais, cerca de $10 \%$ dos pacientes com o complexo da esclerose tuberosa não têm a mutação identificada no laboratório. Isso se deve à limitaçăo técnica.

(i) Ao final desta pesquisa, poderei tomar conhecimento dos resultados, em consulta com a responsável pelo projeto, quando terei a oportunidade de esclarecimentos e aconselhamento genético. Em relação a esta alternativa, minha opção é:

( ) Desejo conhecer os resultados desta pesquisa.

( ) Não desejo conhecer os resultados desta pesquisa.

(j) Tenho a liberdade de desistir e retirar minha participação deste estudo a qualquer momento, sem necessidade de explicaçăo.

(k) Minha participaçăo ou minha desistência năo resultará em ônus ou compensação financeira, năo implicará em interrupçăo ou recusa de atendimento médico nem me trará benefício terapêutico ou assistencial médico.

(I) O DNA ficará sob a responsabilidade do Instituto de Biociências da Universidade de Sâo Paulo e sob a guarda da pesquisadora responsável pela pesquisa, Profa. Dra. Luciana Amaral Haddad, pelo tempo necessário para a identificação e caracterização das mutaçōes relacionadas ao complexo da esclerose tuberosa.

\section{Assinale com um X uma das duas alternativas:}

( ) Concordo que imagens fotográficas obtidas ao meu exame médico ou de exames de imagem sejam utilizadas para fins de publicações científicas, aulas ou seminários científicos sobre o complexo da esclerose tuberosa, mantendo-se sempre a confidencialidade sobre minha identidade.

( ) Não concordo que imagens fotográficas obtidas ao meu exame médico ou de exames de imagem sejam utilizadas para fins de publicaçôes cientificas, aulas ou seminários cientificos sobre o complexo da esclerose tuberosa.

de de 20

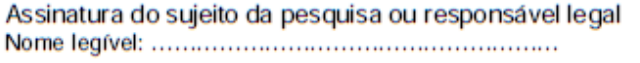
Nome legivel:
Assinatura do pesquisador

Profa. Dra Ludiana Amaral Haddad

Médica, CRM 22803

Telefone: 11-3091-0971 


\section{Appendix 2}

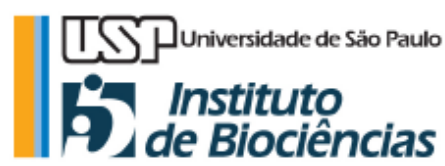

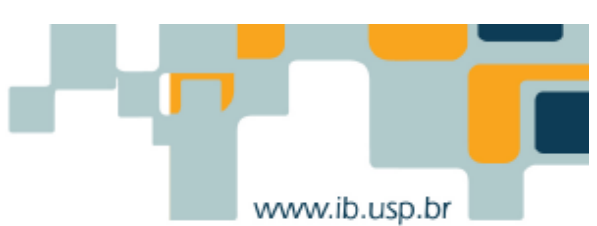

Nùmero de Identificação: LGF

Projeto de pesquisa: Análise dos genes causadores do complexo da esclerose tuberosa

TERMO DE CONSENTIMENTO LIVRE E ESCLARECIDO

(Menores de 18 anos ou indivíduos que năo podem assumir a responsabilidade de assinar) COLETA DE SANGUE

Seu/sua filho(a) ou o(a) paciente sob sua responsabilidade está sendo convidado(a) a participar do projeto de pesquisa acima citado. Este documento contém todas as informaçōes necessárias sobre a pesquisa que estamos fazendo. Sua colaboração neste estudo será muito importante para entendermos um pouco mais sobre o complexo da esclerose tuberosa.

$\mathrm{Eu}$, residente e domiciliado(a) na

inscrito(a) no CPF e portador(a) da cédula de identidade RG............................, declaro que fui esclarecido(a) pelo pesquisador, entendi o que me foi explicado e tive oportunidade para Ihe fazer perguntas sobre o projeto "Análise dos genes causadores do complexo da esclerose tuberosa".

Consinto de livre e espontânea vontade que sob minha responsabilidade, nascido(a) em pesquisa "Análise dos genes causadores do complexo da esclerose tuberosa".

Estou ciente que:

(a) O objetivo do estudo é identificar mutaçōes associadas ao complexo da esclerose tuberosa e entender como a esclerose tuberosa se manifesta.

(b) Será feita coleta de sangue (cerca de 10 mililitros) de veia do(a) paciente, o que poderá causar pequeno incômodo ou dor no momento da coleta (introdução da agulha na veia).

(c) O sangue se destinará à extração de DNA, que deverá ser armazenado em laboratório pelo tempo necessário e utilizado em pesquisa de mutaçōes associadas ao complexo da esclerose tuberosa.

(d) Uma alíquota codificada (sem identificação) do DNA em quantidade suficiente para esta análise poderá ser enviada a um laboratório especializado no exterior.

(e) A análise do sequenciamento do DNA será realizada somente para mutações causadoras e modificadoras do complexo da esclerose tuberosa.

(f) Resultados dos exames clínico e laboratoriais do(a) paciente poderăo ser utilizados para fins de publicaçōes cientificas, aulas ou seminários científicos. No entanto, a publicaçăo de imagens fơtográficas só será possivel com meu consentimento abaixo. 


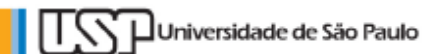 \\ If instutuo de Biociências}

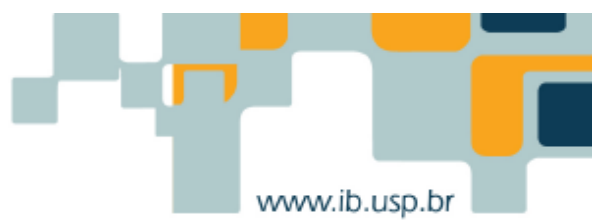

(g) Mutaçōes em qualquer segmento dos genes TSC1 ou TSC2 podem causar a esclerose tuberosa, o que faz a análise laboriosa e demorada. Por este motivo e por se tratar de um novo projeto de pesquisa, os resultados poderão ser obtidos nos próximos anos.

(h) Nas condiçôes atuais, cerca de $10 \%$ dos pacientes com o complexo da esclerose tuberosa năo têm a mutação identificada no laboratório. Isso se deve à limitação técnica.

(i) Ao final desta pesquisa, poderei tomar conhecimento dos resultados, em consulta com a responsável pelo projeto, quando terei a oportunidade de esclarecimentos e aconselhamento genético. Em relação a esta alternativa, minha opção é:

( ) Desejo conhecer os resultados desta pesquisa.

( ) Não desejo conheœr os resultados desta pesquisa.

(j) Tenho a liberdade de desistir e retirar a participaçăo deste estudo a qualquer momento, sem necessidade de explicação.

(k) A participação do(a) paciente ou desistência não resultará em ônus ou compensaçăo financeira, não implicará em interrupção ou recusa de atendimento médico nem Ihe trará benefício terapêutico ou assistencial médico.

(I) O DNA ficará sob a responsabilidade do Instituto de Biociencias da Universidade de São Paulo e sob a guarda da pesquisadora responsável pela pesquisa, Profa. Dra. Luciana Amaral Haddad, pelo tempo necessário para a identificaçăo e caracterizaçăo das mutaçōes relacionadas ao complexo da esclerose tuberosa.

\section{Assinale com um $\mathrm{X}$ uma das duas alternativas:}

( ) Concordo que imagens fotográficas obtidas ao exame médico ou de exames de imagem do(a) paciente sob minha responsabilidade sejam utilizadas para fins de publicaçôes cientificas, aulas ou seminários cientificos sobre o complexo da esclerose tuberosa, mantendo-se sempre a confidencialidade sobre sua identidade.

( ) Não concordo que imagens fotográficas obtidas ao exame médico ou de exames de imagem do(a) paciente sob minha responsabilidade sejam utilizadas para fins de publicações cientificas, aulas ou seminários cientificos sobre o complexo da esclerose tuberosa.

de de 20

\section{Assinatura do sujeito da pesquisa ou responsável legal Nome legivel:}

Assinatura do pesquisador

Profa. Dra Ludiana Amaral Haddad Médica, CRM 22803

Telefone: 11-3091-0971 
Appendix 3

\title{
Understanding human DNA variants affecting pre-mRNA splicing in the NGS era
}

\author{
Luiz Gustavo Dufner-Almeida ${ }^{a}$, Ramon Torreglosa do Carmo ${ }^{b}$, \\ Cibele Masotti ${ }^{\mathrm{b}}$, Luciana Amaral Haddad ${ }^{\mathrm{a}, *}$ \\ ${ }^{a}$ Human Genome and Stem Cell Research Center, Department of Genetics and Evolutionary Biology, Instituto \\ de Biociências, Universidade de São Paulo, São Paulo, Brazil \\ bInstituto Sírio-Libanês de Ensino e Pesquisa, Hospital Sírio-Libanês, São Paulo, Brazil \\ *Corresponding author: e-mail address: haddadl@ usp.br
}

\section{Contents}

1. Introduction

2. Pre-mRNA splicing

2.1 Co-transcriptional exon definition and pre-mRNA splicing 46

2.2 The extended intron definition 48

2.3 Cajal bodies and nuclear speckles: snRNP biogenesis, maturation and storage

3. DNA variants affecting pre-mRNA splicing

3.1 Classical SV: DNA variants in canonical splicing sites

3.2 The minigene approach and gene expression analyses for functional assessment of SV at the mRNA level

3.3 "Miscalled" missense, nonsense and synonymous DNA variants?

3.4 Deep intronic SV 64

3.5 Interfering in exon skipping by antisense oligonucleotide 69

4. Pre-mRNA splicing alterations and cancer 70

4.1 Alternative splicing and the hallmarks of cancer 70

4.2 Somatic mutations affecting cis- and trans-regulatory factors in cancer $\quad 71$

4.3 Genetic basis of alternative splicing in cancer $\quad 74$

5. Concluding remarks 76

$\begin{array}{ll}\text { Acknowledgments } & 77\end{array}$

References

Abstract

Pre-mRNA splicing, an essential step in eukaryotic gene expression, relies on recognition of short sequences on the primary transcript intron ends and takes place along transcription by RNA polymerase II. Exonic and intronic auxiliary elements may modify the strength of exon definition and intron recognition. Splicing DNA variants (SV) have been associated with human genetic diseases at canonical intron sites, as well as exonic 


\title{
Appendix 4
}

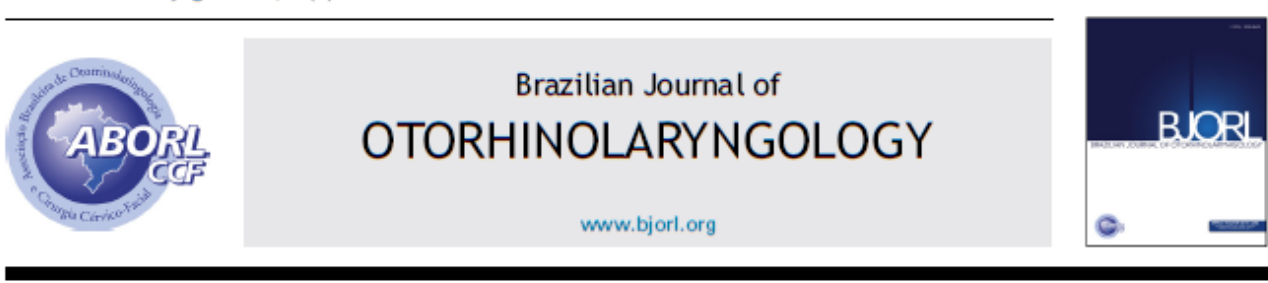

REVIEW ARTICLE

\section{Stem-cell therapy for hearing loss: are we there yet?}

Luiz Gustavo Dufner-Almeida (1) a, Dayane Bernardino da Cruz (B) a, Regina Célia Mingroni Netto ${ }^{[}$a , Ana Carla Batissoco $\mathbb{C}^{\mathrm{b}}$, Jeanne Oiticica $\mathbb{C}^{\mathrm{b}}$, Rodrigo Salazar-Silva (ib) a,*

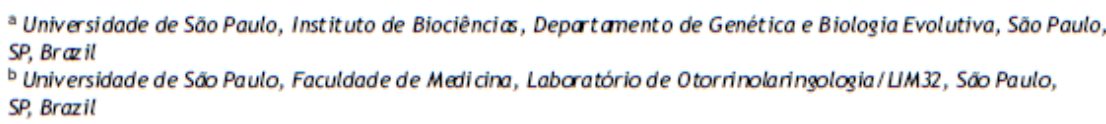

\section{KEYWORDS}

Cellular therapy;

Stem cells;

Hair cells;

Auditory neurons

\begin{abstract}
Introduction: Mammalian hair cells and auditory neurons do not show regenerative capacity. Hence, damage to these cell types is permanent and leads to hearing loss. However, there is no treatment that re-establishes audit ory function. Regenerative therapies using stem cells represent a promising alternative.

Objective: This article ai ms to review the current literat ure about the main types of stem cells with potential for application in cell therapy for sensorineural hearing loss, the most relevant experiments already performed in animals, as well as the advances that have been recently made in the field.

Methods: Research included the databases PubMed/MED. INE, Web of Science, Science Direct and SCIELO, as well as gray literature. Search strategy induded the foll ow ing main terms: "stem cells", "hair cells" and "auditory neurons". Additionally, the main terms were combined with the following secondary terms: "mesenchymal", "iPS", "inner ear", "auditory". The research was conducted independently by three researchers.

Results: Differentiation of stem cells into hair cells and auditory neurons has a high success rate, reaching up to $82 \%$ for the first and $100 \%$ for the latter. Remarkably, these differentiated cells are able to interact with hair cells and auditory neurons of cocliea explants through formation of new synapses. When transplanted int o the cochlea of animals with hear ing loss, auditory restoration has been documented to date only in deafferented animals.
\end{abstract}

\footnotetext{
Thease cite this article as: Dufner-Almeida LG, Cruz DB, Mingroni Netto RC, Batissoco AC, Oitidica J, Salazar-Silva, R. Stem-cell therapy for hearing loss: are we there yet? Braz J Otorhinolaryngd. 2019;85:520-9.

Corresponding author.

E-mail: rodrigosalazarsilva@hotmail.com $\mathbb{R}$. Salazar-Silva).

https:// doi.org/ 10.1016/j.bjort.2019.04.006

1808-8694/ O 2019 Assodiação Brasileira de Otorrindaringologia e Cirurgia Cévico-Facial. Published by Elsevier Editora Ltda. This is an open access article under the CC BY license (httt:// crea tivecommons. org/licerses/ by/4.0/).
} 